\title{
Sicherheit vor Pestiziden in Europa? \\ - Gefährliche Formen der Zusammenarbeit von EU und Mitgliedstaaten am Beispiel der Neu-Genehmigung von Glyphosat
}

\author{
Dagmar Richter*
}

Inhalt

A. Einführung 221

I. Glyphosat - ein exemplarisches Breitbandherbizid 221

II. Basisinformationen 223

1. Verbreitung und wirtschaftliche Bedeutung glyphosathaltiger Pflanzenschutzmittel - sind sie ersetzbar?

2. Gefahrenpotential 228

B. Zuständigkeiten und Verteilung der Entscheidungsmacht 233

I. Genehmigung des Wirkstoffs durch die EU 234

1. Zuständigkeit der EU und Form der Genehmigung: Die Durchführungsverordnung (EU) 2017/2324 zur Erneuerung der Genehmigung des Wirkstoffs Glyphosat 234

2. Einbindung der Mitgliedstaaten im Komitologie-Verfahren 235

a) Fachausschuss und Berufungsausschuss für Phytopharmazeutika 235

b) Die Entscheidung vom 27. November 2017 zur Verlängerung der Genehmigung von Glyphosat

3. Die Rolle des Europäischen Parlaments 237

II. Zulassung glyphosathaltiger Pflanzenschutzmittel durch die Mitgliedstaaten am Beispiel Deutschlands

1. Entscheidungsstrukturen auf Bundesebene 239

2. Entscheidungsstrukturen auf Länderebene und Verzahnung mit Bundesinstitutionen

C. Genehmigungsverfahren und Risikobewertung 242

I. EU-Wirkstoffzulassung 242

1. Ablauf des Genehmigungsverfahrens und Mechanismus der Risikobewertung 242

2. Maßstäbe der Risikobewertung 245

a) Anforderungen an den Wirkstoff 246

b) Anforderungen an mindestens ein Pflanzenschutzmittel, das den Wirkstoff enthält („repräsentative Formulierung“)

* Prof. Dr. Dagmar Richter, Universität Heidelberg, Universität des Saarlandes. 
c) Bewertungsgrundsätze, insbesondere Grundsätze der Transparenz und Wissenschaftlichkeit

3. Die Entscheidung zur Erneuerung der Genehmigung im Fall des Wirkstoffs Glyphosat

a) Risikobewertung 254

b) Inhalt der erneuerten Genehmigung 260

aa) Durchführungsverordnung Glyphosat 260

bb) Folgeentscheidungen: Erlaubte Rückstände von Glyphosat in Lebens- und Futtermitteln

c) Konnte nur durch die Zustimmung Deutschlands der Glyphosatverbrauch eingeschränkt werden?

4. Der Streit um die Risikobewertung 268

a) Die Portier-Kontroverse 268

b) Kritische Fragen zur Rolle des BfR im Deutschen Bundestag 270

c) Erkenntnisse aus Rechtsstreitigkeiten in den USA: Die "Monsanto Papers“

d) Schlussfolgerungen zur Unabhängigkeit und Wissenschaftlichkeit der Bewertung

II. Pflanzenschutzmittelzulassung durch die Mitgliedstaaten, insbesondere Deutschland

1. Europäische Vorgaben 276

a) Grundlegende Anforderungen und „zonales Verfahren“ 276

b) Verweigerung der Zulassung und Zulassung unter Auflagen 277

c) Erneuerung der Zulassung 280

2. Deutsche Regelungen zur Pflanzenschutzmittel-Zulassung 281

3. Neuzulassung glyphosathaltiger Mittel 284

a) Neuzulassungsanträge in Deutschland 284

b) Vorstöße zur Einschränkung der Nutzung glyphosathaltiger Mittel im EU-weiten Vergleich

c) Vereinbarkeit von „Reduktionsstrategien“ (Verboten) mit den europäischen Vorgaben zur Neuzulassung glyphosathaltiger Pflanzenschutzmittel

D. Fortlaufende Kontrolle und Handlungsoptionen 295

I. Europäische Ebene 296

1. Überprüfung der Wirkstoffgenehmigung 296

2. Überprüfung der Pflanzenschutzmittel-Zulassung 296

3. Notfallmaßnahmen 296

4. Aktionsrahmen für die nachhaltige Verwendung von Pestiziden 297

II. Mitgliedstaatliches Recht (Deutschland) 298

1. Widerruf und Rücknahme von Pflanzenschutzmittel-Zulassungen 298

2. Nationaler Aktionsplan zur nachhaltigen Anwendung von Pestiziden 300 
3. Verkehrs- und Anwendungskontrollen, insbesondere das „Pflanzenschutz-Kontrollprogramm“

E. Die Neugenehmigung von Glyphosat im Lichte übergeordneter Maßstäbe und widerstreitender Individualrechte

I. Vorsorgeprinzip 304

1. Inhalt und Anforderungen 304

2. Beachtung des Vorsorgeprinzips bei der Erneuerung der Glyphosatgenehmigung?

II. Individualrechte 311

1. Umweltinformationsrechte 311

2. Grund- und Menschenrechte 313

a) Vorsorgeprinzip und Pflicht zur Sicherstellung eines „hohen Gesundheitsniveaus" (Art. 35 Satz 2 GrCh) als Leitprinzipien der Grundrechtsinterpretation

b) Recht auf Gesundheitsvorsorge (Art. 35 Satz 1 GrCh) 315

c) Recht auf Schutz vor schwerwiegenden Umweltbeeinträchtigungen (Art. 7 GrCh, Art. 8 EMRK) 316

d) Recht auf einen pestizidfreien Körper und Schutz vor Kontamination (Art. 3, 7 GrCh, Art. 8 EMRK) 317

III. Prozessuale Durchsetzung und laufende Verfahren 319

F. Ergebnisse $\quad 320$

I. Mängel des Systems 320

II. Bewertung der Situation und Ausblick auf Neues auf dem „Shikimate pathway"

\section{A. Einführung}

\section{Glyphosat - ein exemplarisches Breitbandherbizid}

Das Unkrautvernichtungsmittel „Glyphosat“ scheint zu jenen Wundermitteln zu gehören, die einen Siegeszug um die Welt antreten, bevor man ihre Nebenwirkungen wahrnehmen möchte. Wir wissen allerdings noch nicht, ob es sich am Ende in eine Reihe mit DDT, PCB oder Asbest einordnen lassen wird. Der vorliegende Beitrag zeigt anhand von Glyphosat, wie dieser Wirkstoff und seine Anwendungsprodukte genehmigt bzw. zum Gebrauch zugelassen wurden. Er ist aber als exemplarische Bestandsaufnahme zu verstehen, die klären soll, ob die vorhandenen Regelungsstrukturen einen sicheren und umweltschonenden Umgang mit Pflanzenschutzmitteln bzw. Pestiziden in Europa gewährleisten. Sollte Glyphosat in Zukunft durch einen anderen Wirkstoff ersetzt werden, werden sich vergleichbare Probleme wie die hier gezeigten ergeben. Die Rede ist von einem hoch- bis überkomplexen Regelungssystem, in dem EU-Recht und mitgliedstaatliches Recht sich ergänzen und überschneiden, und das 
sich in einer Verwaltungszusammenarbeit zwischen EU und Mitgliedstaaten fortsetzt, die Verantwortlichkeiten verunklart und sich zunehmend der Kontrolle entzieht.

"Glyphosat" ist die Wirkstoffbezeichnung für ein sogenanntes nicht-selektives Breitspektrum- oder Totalherbizid. Es geht um ein chemisches Mittel, ${ }^{1}$ das nahezu alle Pflanzen vernichtet, indem es ein Enzym (EPSP[S]) hemmt, das nur bei Pflanzen vorkommt und das diese für die Biosynthese bestimmter Aminosäuren essentiell benötigen. ${ }^{2}$ Schon 1950 war es vom Schweizer Wissenschaftler Henri Martin für Cilag synthetisiert worden. Aber erst Anfang der Siebziger Jahre gelang es, eine Herstellungsmethode zu entwickeln, die den Wirkstoff zum landwirtschaftstauglichen Herbizid machte. Das entsprechende Patent wurde 1974 in den USA zugunsten der Monsanto Corporation (inzwischen Teil der Bayer AG) erteilt, ${ }^{3}$ die es noch im selben Jahr unter dem Handelsnamen „Roundup“ auf den Markt brachte. ${ }^{4}$ Nach dem Auslaufen des Monsanto-Patents gelangten zahlreiche Reformulierungen, etwa durch chinesische oder indische Unternehmen, auf den Markt. ${ }^{5}$ Inzwischen gibt es über 750 glyphosatbasierte Rezepturen (Anwendungsmittel) nebst vergleichbarer Wirkstoffe wie z.B. „Glufosinat“.

Glyphosathaltige Pflanzenschutzmittel können in verschiedenen Stadien des Wachstums verwendet werden. Ihre hauptsächliche Wirkung liegt darin, nahezu alle nicht-resistenten Pflanzen, d.h. nicht nur Unkräuter, sondern auch Nutzpflanzen (!), unterschiedslos zu vernichten. Daneben beschleunigt es die Trocknung vor der Ernte und so die Abreifung: Bei der sogenannten „Sikkation“ wird die Kulturpflanze kurz vor der Ernte durch Versprühen des Herbizids gezielt abgetötet. Theoretisch kann mit Hilfe von glyphosathaltigen Mitteln sogar der Erntetermin routinemäßig gesteuert werden. Da die Verwendung zur Sikkation allerdings als Hauptursache dafür gilt, dass Glyphosat in Lebens- und Futtermittel gelangt, wird sie zunehmend untersagt, wie z.B. in Österreich. ${ }^{6}$

Für die Verwendung von Glyphosat zur Unkrautvernichtung ergeben sich grundsätzlich zwei Möglichkeiten: Entweder das Mittel wird schon vor dem Wachsen der Nutzpflanzen ausgebracht, um den Boden von Unkraut zu befreien (Nutzung mit Vorsaat- und Vorlaufverfahren), oder es wird zusammen mit dem Breitbandherbizid

1 Glyphosat (ISO): N-(Phosphonomethyl)Glyzin mit der Molekularformel: C3H8NO5P; EC-Listennr. 213-997-4; CAS-Nr. 1071-83-6; Index-Nr. 607-315-00-8. Siehe zu den chemischen Eigenschaften die Schlussfolgerungen der Europäischen Behörde für Lebens- und Futtermittelsicherheit (EFSA): EFSA, Conclusion on the peer review of the pesticide risk assessment of the active substance glyphosate, Anhang A (S. 30 f.); Abdruck in: EFSA Journal 2015/13, S. $4302 \mathrm{ff}$.

2 S. Holländer/Amrhein, Plant Physiology 1980/66, S. 823-829.

3 Patent US3799758 (N-phosphonomethyl-glycine phytotoxicant compositions), United States Patent Ser. No. 170,385, veröffentlicht am 26. März 1974, abrufbar unter: https://patents.g oogle.com/patent/US3799758 (15.06.2019).

4 Zur Entstehungsgeschichte des Wirkstoffes Székács/Darvas, in: Mohammed Nagib, MNAEG (Hrsg.), InTech Europe (Croatia) 2012, S. 247-84, abrufbar unter: http://cdn.intechweb. org/pdfs/25624.pdf (15.06.2019).

5 AgroNews vom 23.02.2017 "China's top glyphosate exporters in 2016”), abrufbar unter: http://news.agropages.com (15.06.2019).

6 S.u. D II.1. 
(Glyphosat) ein (glyphosat-)resistentes Saatgut verwendet. Während das 1974 von Monsanto produzierte „Roundup“ in der Anfangszeit hauptsächlich noch dazu diente, Wege und Höfe von Unkraut zu befreien, stieg der Verbrauch in der Landwirtschaft sprunghaft an, nachdem Monsanto die Schaffung glyphosat-resistenter Sojabohnen gelungen war und das entsprechende Saatgut ab 1996 auf den Markt gelangte. ${ }^{7}$ Inzwischen erstreckt sich das Spektrum resistenten Saatguts auf alle gängigen Nutzpflanzen. Dort, wo der Einsatz gentechnisch veränderter Pflanzen erlaubt wurde, entstand ein „Kombi-System“, bei dem landwirtschaftliche Betriebe nicht nur das Unkrautvernichtungsmittel kauften, sondern auch das dazu passende resistente Saatgut.

\section{Basisinformationen}

\section{Verbreitung und wirtschaftliche Bedeutung glyphosathaltiger Pflanzenschutzmittel - sind sie ersetzbar?}

Glyphosathaltige Pflanzenschutzmittel werden in den verschiedensten Bereichen verwendet, nicht nur im eigentlichen Ackerbau, sondern auch im Obst-, Wein- und Hopfenanbau, zur Freihaltung öffentlicher Flächen (z.B. Gleisanlagen, Wege, Spielplätze) von Unkraut oder zum heimischen Gebrauch. Die Liste der Nutzpflanzen, für die solche Mittel verwendet werden, ist lang und enthält neben den typischen Feldund Baumfrüchten z.B. auch Kräuter, Gewürze oder Zierpflanzen. ${ }^{8}$

Wie beliebt Glyphosat im weltweiten Maßstab wurde, belegen die explosionsartig gestiegenen Verkaufszahlen von glyphosathaltigen Produkten wie auch von glyphosat-resistentem Saatgut. Während 1994 noch ca. 56.000 Tonnen Glyphosat (aktiver Wirkstoff) abgesetzt/verbraucht wurden, waren es 2014 ca. 826.000 Tonnen. ${ }^{9}$ Dabei können die Daten allerdings nur hochgerechnet werden, weil transparente Verbrauchslisten nicht geführt werden. ${ }^{10}$ In den Zahlen spiegelt sich dennoch der sprunghafte Anstieg wieder, den die Einführung glyphosat-resistenten Saatguts ab 1996 ausgelöst hatte.

2012 wurde Glyphosat mit über 700.000 Tonnen das in Gestalt von über 750 verschiedenen Produkten meistgenutzte Herbizid überhaupt. Für 2014 kursiert die Zahl von 825.804 Tonnen weltweiten Verbrauchs. ${ }^{11}$ Ein Staat wie Brasilien, der seit 1998 transgenes Soja anbaut, konnte nur mithilfe der neuen Möglichkeiten zum zweit-

7 Benbrook, Environmental Sciences Europe 2016/28, S. 1, abrufbar unter: https://doi.org/1 0.1186/s12302-016-0070-0 (15.06.2019).

8 Siehe etwa die Liste der Europäischen Behörde für Lebensmittelsicherheit (EFSA) in deren "Reasoned Opinion" vom 16.01.2012 ("Modification of the existing MRL for glyphosate in lentils"), Appendix C, abrufbar unter: www.efsa.europa.eu/en/search/doc/2550.pdf (15.06.2019).

9 Benbrook, Environmental Sciences Europe 2016/28, S. 7 (Tabelle 4).

10 Ibid., S. 2-4, 12.

11 FAZ Nr. 182 vom 08.08.2018, S. 15 („Glyphosat droht in Brasilien das Verbot“), unter Hinweis auf eine nicht benannte Studie. 
größten Sojaproduzenten der Welt aufsteigen. ${ }^{12}$ Wie eine Marktforschungsstudie zeigt, soll der Glyphosat-Markt (d.h. die durchschnittliche jährliche Investitionsrate [Compound Annual Growth Rate]), im Zeitraum zwischen 2016 und 2022 um 6.32\% wachsen, so dass er 2022 ein errechnetes Volumen von 9.91 Milliarden USD erreichen würde. ${ }^{13}$

Soweit es Deutschland im Speziellen betrifft, veröffentlicht das Julius-Kühn-Institut zwar im Rahmen der jährlichen PAPA (Panel Pflanzenschutzmittel-Anwendungen)- Erhebungen ein „Wirkstoffranking“ in Bezug auf ausgewählte Kulturpflanzen. Daraus ergibt sich z.B. für 2017, dass 75,7\% aller Erhebungsbetriebe Glyphosat für den Weinbau oder $86,3 \%$ es für die Apfelproduktion einsetzten. ${ }^{14}$ Soweit es die Wirkstoffmengen betrifft, finden sich hier aber nur hochgerechnete Schätzwerte für Gesamtdeutschland, und zwar mit der ausdrücklichen Einschränkung, dass die Werte „aufgrund der geringen Stichprobengröße“ höchst unsicher seien. Infolgedessen gibt das Institut weit auseinanderklaffende Unter- und Obergrenzen und einen dazu errechneten Schätzwert an, z.B. 2017 ca. $24.700 \mathrm{~kg}$ Glyphosat für Apfel, $55.550 \mathrm{~kg}$ für Wein, ca. $58.800 \mathrm{~kg}$ für Kartoffeln, $95.0925 \mathrm{~kg}$ für Mais oder $1.031 .442 \mathrm{~kg}$ für Winterweizen. Die Bundesregierung beruft sich darauf, dass es „keine Rechtsvorschrift zur Erfassung und Meldung der Anwendungsmengen im EU-Pflanzenschutzrecht oder im deutschen Pflanzenschutzgesetz" gäbe. ${ }^{15}$

Auch aus dem Bereich des Pflanzenhandels kommen keine präzisen Daten. Zwar besteht gemäß $\$ 64$ Pflanzenschutzgesetz (PflSchutzG) ${ }^{16}$ eine Meldepflicht hinsichtlich der Art und Menge der an Empfangsstellen mit (Wohn-)Sitz im Inland abgegebenen oder ausgeführten Pflanzenschutzmittel und der jeweils in ihnen enthaltenen Wirkstoffe nebst sonstiger Ingredenzien („Safener“ und „Synergisten“), ${ }^{17}$ jeweils getrennt für jedes Pflanzenschutzmittel sowie nach beruflichen und nicht-beruflichen Verwendern. Der tatsächliche Absatz an Pflanzenschutzmitteln in Deutschland wird

12 S. bereits Bericht des Ausschusses für Bildung, Forschung und Technikfolgenabschätzung (18. Ausschuss) gemäß \56a der Geschäftsordnung „Transgenes Saatgut in Entwicklungsländern - Erfahrungen, Herausforderungen, Perspektiven“, BT-Drs. 16/13874 vom 06.08.2009, S. 62 f., 65.

13 MarketsandMarkets Research Private Ltd., Summary zur Studie "Glyphosate Market by Crop Type (Genetically Modified, Conventional), Form (Liquid, Dry), Application (Agricultural (Grains \& Cereals, Oilseeds \& Pulses, Fruits \& Vegetables), Non Agricultural, and Region - Global Forecast to 2022”, abrufbar bei AgReport vom 22.02.2017 unter: http:// doc.agropages.com (15.06.2019).

14 Siehe PAPA - Seite des Julius-Kühn-Instituts unter https://papa.julius-kuehn.de/ (15.06.2019). Zum Auftrag des JKI im Bereich der Pestizid-Statistiken noch u. D II.2.

15 Antwort der BReg auf die Kleine Anfrage der Abgeordneten Dr. Kirsten Tackmann u.a. und der Fraktion „Die Linke“(,Wirkung von Glyphosat auf Säugetiere, Insekten und Wirbeltiere“), BT-Drs. 19/4915 vom 11.10.2018, S. 7.

16 Gesetz zum Schutz der Kulturpflanzen vom 06.02.2012 (BGBl. I S. 148, 1281), zuletzt geändert durch Art. 4 Abs. 84 des Gesetzes vom 18.07.2016 (BGBl. I S. 1666).

17 S. zu beiden Begriffen die Legaldefinitionen in Art. 2 Abs. 3 VO (EG) 1107/2009. 
danach auch jährlich in einem Meldebericht veröffentlicht. ${ }^{18}$ Dort erfährt man bei den aktuellen Meldungen für 2017 aber nur, dass Glyphosat in der „Mengenklasse“ zwischen 2.500-10.000 Tonnen/Jahr liege und damit nach Kohlendioxid das am zweitstärksten verbreitete Mittel beim Inlandsabsatz für berufliche Verwender sei. ${ }^{19}$ Überall dort, wo der Bericht konkrete Tonnenangaben macht, ist dagegen nur von Wirkstoffgruppen wie „Herbiziden“, „Organophosphor-Herbiziden“ oder „Sonstigen Herbiziden“ die Rede, so dass für die interessierte Öffentlichkeit unklar bleibt, wieviel Tonnen Glyphosat im Inland bezogen wurden und wie sich die Menge über die Jahre hinweg entwickelt hat. ${ }^{20}$ Nur Eingeweihte wissen, dass sich unter der Bezeichnung „Organophosphor-Herbizide“ die Wirkstoffe Glyphosat und Glufosinat sowie der Wachstumsregulator Etephon aus der Gruppe der Phytohormone verbergen. ${ }^{21} \mathrm{Da}$ Glufosinat nur aus Deutschland exportiert, aber nicht in Deutschland selbst angewendet wird, ${ }^{22}$ trägt es wahrscheinlich nicht zur Verunklarung der Inlandsabsatzzahlen für Glyphosat bei, was aber wohl im Hinblick auf Etephon der Fall bleibt. Die Meldeberichte des BVL ${ }^{23}$ enthalten für den Inlandsabsatz von OrganophosphorHerbiziden, der allein präzise angegeben wird, die folgenden Daten:

2012: 6.038 Tonnen (beruflich: 5.998, privat: 40)

2013: 5.113 Tonnen (beruflich: 5.040, privat: 73 )

2014: 5.472 Tonnen (beruflich: 5.377, privat: 95 )

2015: 4.404 Tonnen (beruflich: 4.330, privat: 74 )

2016: 3.780 Tonnen (beruflich: 3.734, privat: 46 )

2017: 4.694 Tonnen (beruflich: 4.655, privat: 39 )

Lediglich auf parlamentarische Anfragen hin enthüllte die Bundesregierung die Klardaten speziell für den Inlandsabsatz von Glyphosat, ${ }^{24}$ so dass bis 2016 folgende „Klardaten“ verfügbar sind:

18 Die Mengenberichte sind auf der Seite des Bundesamtes für Verbraucherschutz und Lebensmittelsicherheit (BVL) von 2002-2017, abrufbar unter: www.bvl.bund.de/DE/04_Pfl anzenschutzmittel/01_Aufgaben/02_ZulassungPSM/03_PSMInlandsabsatzExport/psm_P SMInlandsabsatzExport_node.html (15.06.2019).

$19 B V L$, Absatz an Pflanzenschutzmitteln in der Bundesrepublik Deutschland, Ergebnisse der Meldungen gemäß \64 Pflanzenschutzgesetz für das Jahr 2017, (Fn. 18), S. 15 (Tabelle 3.4), S. 21 (Tabelle 3.5).

20 S. zu den ähnlich konzipierten europäischen Vorgaben zu Pestizid-Statistiken noch u. D I. 4.

21 Spektrum der Wissenschaft, Lexikon der Chemie, Stichwort „Organophophorherbizide“, abrufbar unter: www.spektrum.de/lexikon/chemie/ (15.06.2019).

$22 B V L$, Meldungen für 2017, (Fn. 18): Danach ist Glufosinat nur in der Ausfuhrrubrik in Tabelle 3.4. zu finden.

23 Siehe Fn. 18.

24 Antwort der Bundesregierung auf die Kleine Anfrage der Abgeordneten Carina Konrad u.a. und der Fraktion der FDP, BT-Drs. 19/4097 vom 28.08.2018, S. 2. 
2010: 5.007 Tonnen (ohne Aufschlüsselung)

2011: 5.359 Tonnen (ohne Aufschlüsselung)

2012: 5.981 Tonnen (davon privat: 40 )

2013: 5.065 Tonnen (davon privat: 73 )

2014: 5.426 Tonnen (davon privat: 95 )

2015: 4.315 Tonnen (davon privat: 74 )

2016: 3.780 Tonnen (davon privat: 46 )

Der Bundesregierung liegen weder über die tatsächlich ausgebrachten Mengen Daten vor noch kann sie angeben, in welchen Einsatzgebieten glyphosathaltige Mittel wie stark verwendet werden; bekannt ist nur, dass die Deutsche Bahn 2015 77,5 t, 2016 67,6 t und 2017 65,4 $\mathrm{t}$ auf ihren Anlagen ausbrachte. ${ }^{25}$ Vergleicht man die beiden Datentabellen, beträgt der Anteil von Glyphosat an den Organophosphor-Herbiziden zwischen 97 und 100\%. Eine Art Höhepunkt erreichte die Absatzmenge in Deutschland 2012, während sie bis einschließlich 2016 deutlich sank, um dann 2017 wieder erheblich anzusteigen. Warum das so ist, kann hier nicht verlässlich beurteilt werden. Sehr wahrscheinlich ist aber, dass politische Forderungen nach dem „Glyphosatausstieg" und ein mögliches Scheitern der Ende 2017 anstehenden Wiedergenehmigung ${ }^{26}$ des Wirkstoffs Glyphosat zu einer Vorratsbeschaffung bei den beruflichen Verwendern geführt hatten. Gegenläufig zur Entwicklung der Absatzmenge von Glyphosat bei der beruflichen Verwendung verblieb die Absatzmenge bei der privaten Nutzung aber auch $2017 \mathrm{klar}$ im Abwärtstrend. Beides dürfte sich gleichermaßen mit der zunehmenden öffentlichen Kritik an Glyphosat erklären. Denn während landwirtschaftliche Betriebe oder auch die Deutsche Bahn als größter professioneller Einzelverbraucher ${ }^{27} 2017$ das Versiegen der Glyphosatquellen und damit Umsatzeinbußen befürchten mussten, dürften private Nutzer und Nutzerinnen nicht unbeeindruckt von der Möglichkeit geblieben sein, eine vielleicht krebserzeugende Substanz im eigenen Garten zu verteilen.

Die Frage, ob die Welt ohne Glyphosat oder sogar ohne Pestizide auskommen könnte, wird je nach Interessenlage unterschiedlich beantwortet. Die UN-Sonderberichterstatterin für das Recht auf Nahrung widerspricht entschieden der Behauptung, die Welternährung könne nur auf der Grundlage von Pestiziden gesichert werden. ${ }^{28}$ Auch Institutionen, die den Glyphosateinsatz befürworten, räumen ein, dass es Alternativen zu glyphosathaltigen Mitteln gibt, z.B. den Einsatz von Mikroorganismen oder „besserer" Chemikalien, aber auch Fruchtwechsel und mechanische Methoden

25 BReg, (Fn. 24), S. 2.

26 S.u. B I.2.b).

27 Die Deutsche Bahn versprüht aktuell ca. 75 Tonnen Glyphosat pro Jahr zur Unkrautbekämpfung am Gleisbett, was allerdings nur ca. 0,4 Prozent der Gesamtmenge ausmacht. Siehe Handelsblatt vom 18.12.2018, S. 2 („Glyphosat - Letzte Runde im Kampf um das Umweltgift. Größter Einzelverbraucher ist die Bahn“).

28 UN General Assembly, Report of the Special Rapporteur (Hilal Elver) on the right to food, A/HRC/34/48 vom 24.01.2017, $\$ \$ \$ 90 \mathrm{ff}$. 
wie „Pflügen“ bzw. thermische Einwirkung auf Verkehrsflächen. ${ }^{29}$ Das oft genannte Argument, im Falle eines Glyphosatverbots könnten Chemikalien eingesetzt werden, die am Ende viel giftiger als Glyphosat seien, überzeugt zwar nicht, weil auch sie den Anforderungen an den Schutz der Gesundheit und Umwelt entsprechen müssen. Dass alternative Mittel aber mit anderen Nachteilen, vor allem wirtschaftlichen, verbunden sind, ist unbestreitbar. Unternehmensnahe Studien stellen z.B. für den Fall eines Glyphosatverbots für Einzelagrarbetriebe in Deutschland je nach Anbauprodukt Rückgänge des Deckungsbeitrags ${ }^{30}$ um $40-70 \%$ pro Hektar in Aussicht, die je nach Wertigkeit des Produkts wie z.B. im Falle des Weinbaus einen Verlust in der Größenordnung von 220 Euro pro Hektar bewirken sollen. ${ }^{31} \mathrm{Im}$ Weinbau soll die mechanische Bekämpfung des Unkrauts mittels Freischneider die Kosten um den Faktor zehn und mehr erhöhen. ${ }^{32}$ Nicht zu unterschätzen ist auch der Umstand, dass die jahrzehntelange Nutzung eines Breitbandherbizids wie Glyphosat inzwischen auch die landwirtschaftliche Kultur umgeformt hat. Vor allem dort, wo Glyphosat kombiniert mit glyphosatresistentem Saatgut verwendet wird, hat sich das ganze landwirtschaftliche System von der Zeitplanung bis zum Arbeitskräfte- und Maschinenbedarf auf diese Produktionsmethode eingestellt.

Die kulturelle Gewöhnung an die preiswerte Produktionsform geht Hand in Hand mit einer wachsenden Abhängigkeit der landwirtschaftlichen Betriebe. Sofern sie glyphosatresistentes Saatgut verwenden, müssen sie sich verpflichten, kein Saatgut aus der eigenen Ernte für die kommende Aussaat zu generieren oder aber Lizenzgebühren zu zahlen. ${ }^{33}$ Auch das verändert die Landwirtschaft. Zugleich sind die Möglichkeiten der Auswahl zwischen verschiedenen Anbietern durch Monopolstrukturen beschränkt. Denn nach dem Kauf von Monsanto durch Bayer verfügen nur noch drei global agierende Konzerne über 61\% des weltweit verfügbaren Saatguts und 71\% der agrochemischen Produkte. ${ }^{34}$ Dies hat wiederum zur Folge, dass drei Konzerne den maßgeblichen Einfluss auf die Welternährung nehmen. Wie Medien schon seit längerem beobachten, drängen pestizidherstellende Unternehmen wie insbesondere Monsanto/Bayer zurzeit in die Entwicklungszusammenarbeit, um z.B. im Rahmen der Neuen Allianz für Ernährungssicherheit die Koppelung von Hilfen der G7-Staaten an den Patentschutz zu binden, d.h. an ein Verbot des Tausches von Saatgut in den Saatgutgesetzen der Empfängerstaaten. ${ }^{35}$

29 Bundesministerium für Ernährung und Landwirtschaft, Fragen und Antworten zu Glyphosat (Rubrik „Pflanzenbau“ - „Pflanzenschutz“), abrufbar auf der Homepage des BMEL unter: www.bmel.de (10.01.2019). S.a. Ranzenmayr, S. $26 \mathrm{ff}$.

30 Differenz zwischen Umsatz und variablen Kosten, d.h. verfügbarer Betrag zur Deckung der Fixkosten.

31 Siehe Fairclough et al., S. 31, 39, abrufbar unter: https://www.glyphosate.eu/system/files/ sidebox-files/the_economic_relevance_of_glyphosate_in_germany_24_08_2017.pdf (15.06.2019).

32 Antwort der BReg, BT-Drs. 19/4097 vom 28.08.2018, (Fn. 24), S. 5.

33 Der Spiegel Nr. 13 vom 24.03.2018, S. 78 („Monster-Hochzeit“).

34 Der Spiegel Nr.13 vom 24.03.2018, S. 80: Bayer/Monsanto, ChemChina/Syngenta und Dow/DuPont.

35 Der Spiegel Nr. 13 vom 24.03.2018, S. 82. 


\section{Gefahrenpotential}

Bis heute wird Glyphosat als „Jahrhundert-Herbizid“ (Stephan O. Duke) gepriesen: Es blockiere lediglich „ein Zahnrad im Pflanzen-Stoffwechsel“ - mehr nicht. Weil das blockierte Enzym EPSP(S) weder bei Menschen noch Tieren vorkomme, habe Glyphosat „nur eine geringe Giftwirkung “ und sei nach internationalen Standards weniger akut toxisch als Kochsalz oder Backpulver. ${ }^{36}$ Seine Gegner sind wiederum überzeugt, dass es die Gesundheit von Mensch und Tier wie auch die Umwelt schädigt. Wissenschaftliche Studien bestätigen beides mit entsprechend gegenläufigen Ergebnissen. Gerichtsverfahren rund um den Erdball enden mit unterschiedlichen Ergebnissen. Daneben finden sich vielerlei gemäßigte Positionen. So hat z.B. Deutschland den Glyphosateinsatz gebilligt. Dennoch vertritt die Bundesregierung nicht die These von der folgenlosen Enzymabschaltung, sondern schließt „davon unabhängig auftretende Wirkungen“ ausdrücklich nicht aus. ${ }^{37}$ Die Vielstimmigkeit und Gegensätzlichkeit der Positionen lässt nicht nur Unsicherheit in der wissenschaftlichen Bewertung erkennen, sondern könnte auch Anzeichen für den Eintritt in eine Übergangs- und Zwischenphase sein, die den allzu unbefangenen Umgang mit Totalherbiziden beendet.

Kaum bestreitbar ist, dass die massenweise Verwendung glyphosathaltiger Produkte Folgen hatte. Es zeigten sich endogene Folgen, die das „System Breitbandherbizid“ betreffen, aber auch exogene Folgen, die über das eigentliche Verwendungssystem hinausreichen. Im Bereich der endogenen Folgen zeichnete sich bald ein Problem des „Resistenzen-Managements“ 38 ab. Denn je mehr glyphosathaltige Pestizide ausgebracht wurden, umso mehr Resistenzen entstanden auch unter den Unkräutern. Es verbreiteten sich - sei es infolge sachgemäßen oder unsachgemäßen Gebrauchs, erwartbar oder durch Verkettung unglücklicher Umstände „Superunkräuter“, gegen die Glyphosat in der vorgesehenen Form nicht mehr half. Die Folge war, dass die Dosis des Vernichtungsmittels erhöht, Glyphosat mit anderen Giften gemischt oder auf kritische Altpräparate (z.B. „Dicamba“) zurückgegriffen wurde. ${ }^{39}$ Inzwischen sind Farmer z.B. in Südamerika darauf angewiesen, dass ihnen Monsanto möglichst jährlich neue Rezepturen liefert, damit die gewünschte Wirkung permanent erzielt werden kann. Führt die Entstehung resistenter Unkräuter aber zur kontinuierlichen Verschärfung oder Dosiserhöhung des Herbizids und dies wiederum zur Entstehung von „Superunkräutern“, die dann immer hartleibiger werden, kann es zu einem eskalierenden „Kreislauf des Schreckens“ kommen.

Die exogenen Folgen sind in höchstem Maße umstritten. Das gilt insbesondere für die Frage, ob glyphosathaltige Pflanzenschutzmittel Gesundbeitsgefabren für Men-

36 Antrag der Abgeordneten Christian Lindner u.a. und der Fraktion der FDP („Nationale Strategie für den Umgang mit dem Herbizid-Wirkstoff Glyphosat“) vom 11.12.2017, BTDrs. 19/216, S. 1.

37 Antwort der BReg auf die Kleine Anfrage der Abgeordneten Harald Ebner u.a. und der Fraktion Bündnis 90/Die Grünen, BT-Drs. 18/5347 vom 29.06.2015, S. 14.

38 Siehe u.a. Heap, Pest Management Science 2014/70, S. 1306-1315; Nandula; Tamms et al, in: Julius-Kühn-Archiv 2018/458, S. 270 ff., stellten Sensitivitätsverluste bei Süßgräsern fest.

39 Auch zum Folgenden Der Spiegel Nr. 13 vom 24.03.2018, S. 81 f. 
schen und Tiere begründen, d.h. vor allem reproduktionstoxische und/oder kanzerogene Wirkungen haben. ${ }^{40}$ Die unübersehbaren Folgen, die der exzessive Glyphosatgebrauch in Südamerika bewirkt hat, werden zumeist mit einem unsachgemäßem Gebrauch erklärt, der in Europa so nicht stattfinde. ${ }^{41}$ Ausgangspunkt für die Risikobewertung sind grundlegende Eigenschaften des Wirkstoffs in der Umwelt. Im Boden wandelt sich Glyphosat durch Mikroorganismen zum Abbauprodukt AMPA (Aminomethyl-Phosphonsäure) um. Während die Halbwertszeit für Glyphosat bei 6 bis 41 Tagen liegt, schwanken die Angaben für AMPA innerhalb einer Bandbreite zwischen 39 und 301 Tagen. ${ }^{42}$ Gesichert erscheint, dass Glyphosat im Boden, in der Luft, Oberflächen- und Grundwasser nachgewiesen werden kann. ${ }^{43}$ Über den Boden kann Glyphosat/AMPA bei ungünstigen Verhältnissen in Wasserläufe und das Grundwasser geschwemmt werden. Im Wasser ist Glyphosat dann „chemisch relativ stabil und auch fotochemisch nicht abbaubar" ${ }^{44}$ Es wirkt langanhaltend toxisch auf Wasserorganismen und trägt deshalb auch einen Warnhinweis. ${ }^{45}$

Von größter Bedeutung für mögliche Gesundheitsgefahren sind die Rückstände von Glyphosat in Lebensmitteln und Trinkwasser. Wie die Stiftung Warentest ermittelte, finden sich inzwischen Spuren von Pflanzenschutzmitteln und AMPA zusammen mit Röntgenkontrast-, Arznei-, Korrosionsschutzmitteln nebst manchem Anderen in Trink- und Mineralwässern. ${ }^{46} \mathrm{Da}$ AMPA auch durch Hydrolyse aus Phosphonsäuren gebildet werden kann und diese auch in Wasch- und Reinigungsmitteln, Kühlkreisläufen etc. vorkommen, ${ }^{47}$ besteht aber fast immer die Möglichkeit, einen Zusammenhang mit der Verwendung glyphosathaltiger Pflanzenschutzmittel zu bestreiten. Aufsehen erregten Untersuchungen der Stiftung Warentest und des Umweltinstituts München e.V., die 2016 und 2017 unter den in Deutschland absatzstärksten Bieren bis zu ca. 30 Mikrogramm Glyphosat pro Liter Bier vorfanden. Das Bundesamt für Risikobewertung stufte dies als ganz normalen Befund ein („Glyphosatrückstände in Bier sind aus wissenschaftlicher Sicht plausibel und grundsätzlich erwartbar“), verortete die Verantwortung dafür in anderen EU-Mitgliedstaaten, wo die Nutzung von Glyphosat zur Sikkation erlaubt sei, und begründete die Unbedenklichkeit des Be-

40 S.u. C I.3. und 4.

41 Antwort der BReg, BT-Drs. 18/5347, (Fn. 37), S. 15.

42 Ranzenmayr, S. 11 m.w.N.

43 IARC, IARC Monographs on the Evaluation of Carcinogenic Risks to Humans, Bd. 112 (2017): Evaluation of five organophosphate insecticides and herbicides, S. 75, abrufbar unter: https://monographs.iarc.fr (15.06.2019).

44 Antwort der BReg, BT-Drs. 18/5347, (Fn. 37), S. 17.

45 Stoffinformation "Glyphosat" (CAS no. 1071-83-6) der Europäischen Chemikalienagentur ECHA, abrufbar unter: https://echa.europa.eu/de/substance-information: "Danger! According to the harmonised classification and labelling (CLPOO) approved by the European Union, this substance is toxic to aquatic life with long lasting effects and causes serious eye damage".

46 Stiftung Warentest Heft 2016/8: Die Stiftung fand AMPA in rund jeder zweiten Trinkwasserprobe und drei Mineralwässern, konnte aber nicht klären, ob die Rückstände von Glyphosat oder Waschmitteln stammten. Wegen der geringen Mengen bestehe kein gesundheitliches Risiko.

47 Antwort der BReg, BT-Drs. 18/5347, (Fn. 37), S. 18. 
fundes damit, dass die zulässigen Grenzwerte um ein Vielfaches unterschritten seien. ${ }^{48}$ Es gibt allerdings keine Erklärung dafür, warum für Trinkwasser ein gesetzlicher Grenzwert von 0,1 Mikrogramm ${ }^{49}$ gilt, für Glyphosat- Rückstände im Bier aber einer von $30 \mathrm{mg}$ (= 30.000 Mikrogramm)/Liter. Insgesamt legen die Erkenntnisse über Glyphosat-Rückstände in Lebensmitteln nahe, dass diese zwar noch überwiegend geringfügig, aber doch zunehmend und breitflächiger auftreten, so dass mit Akkumulationen der Glyphosataufnahme aus unterschiedlichen Lebensmitteln in den kommenden Jahren zu rechnen ist. Dazu trägt auch bei, dass Glyphosat bei hohen Temperaturen stabil bleibt, so dass es z.B. auch noch in fertigen Backwaren nachgewiesen werden kann. ${ }^{50}$

Soweit es die Aufnabme durch den menschlichen Körper betrifft, konnten Studien Glyphosat im Urin und Blut von Menschen nachweisen, die Glyphosat selbst ausbrachten oder in der Nähe besprühter Flächen lebten, aber auch bei städtischer Bevölkerung, die es wohl vornehmlich über die Nahrung aufnahm. ${ }^{51}$ Geringe Mengen an Glyphosat/AMPA wurden in einer nordrhein-westfälischen HBM (Human BioMonitoring) -Studie bei $63 \%$ von 250 untersuchten Kita-Kindern im Urin gefunden. ${ }^{52}$ Diese Befunde werden zum Teil mit dem Argument für unbedenklich erklärt, der Wirkstoff werde „unverändert direkt über die Nieren ausgeschieden“ ${ }^{53}$ Dass dabei keine Verstoffwechselung von Glyphosat im Körper stattfindet, konnte das Bun-

48 BfR, Einschätzung zu Gehalten von Glyphosat in Bier, Aktualisierte Mitteilung Nr.012/2018 des BfR vom 24.05.2018: „... Um gesundheitlich bedenkliche Mengen von Glyphosat aufzunehmen, müsste ein Erwachsener an einem Tag rund 1000 Liter Bier trinken. Glyphosatgehalte von 30 Mikrogramm pro Liter Bier und darunter stellen nach dem derzeitigen Stand des Wissens kein gesundheitliches Risiko dar.", abrufbar unter: www.bfr. bund.de (10.01.2019).

49 Siehe Verordnung über die Qualität von Wasser für den menschlichen Gebrauch (Trinkwasserverordnung) i.d.F. der Bekanntmachung vom 10.3.2016 (BGBl. I S. 459), geändert zuletzt durch Artikel 1 der Verordnung vom 3. Januar 2018 (BGBl. I S. 99). Gemäß Laufender Nr. 10 der Anlage 2 zu $\ 6$ Abs. 2 beträgt der Grenzwert für „PflanzenschutzmittelWirkstoffe und Biozidprodukt-Wirkstoffe", jeweils einzeln betrachtet, $0,00010 \mathrm{mg} / \mathrm{l}$, in der Kombination mehrerer Wirkstoffe $0,00050 \mathrm{mg} / \mathrm{l}$.

50 Stiftung Öko-Test, Glyphosat in Getreideprodukten, Öko-Test 2012/9, S. 46-49; Ratgeber Essen 2013/12, S. 40-43: Der Test zeigte bei acht von zehn untersuchten Brötchen von Supermärkten und Bäckereiketten Glyphosatrückstände zwischen 0,017 und 0,12 mg/kg. Ebenfalls positiv getestet wurden Mehle, Müslis und Haferflocken.

51 IARC, (Fn. 43), S. 321 ff. (Glyphosate), 361 m.w.N., abrufbar unter: https://monographs. iarc.fr (15.06.2019). S.a. Medizinisches Labor Bremen, Determination of Glyphosate residues in human urine samples from 18 European countries, Studie vom 06.06.2013 im Auftrag des BUND, abrufbar unter: www.weltagrarbericht.de (15.06.2019): Die Studie stellt bei einer eher geringen Zahl von Probanden Anhaltspunkte für große Schwankungen der Glyphosatbelastung des Urins städtischer Bevölkerung in den einzelnen Staaten fest.

52 Landesamt für Natur, Umwelt und Verbraucherschutz (LANUV) NRW, Bestimmung von Schadstoffen und Schadstoffmetaboliten im Urin von 2- bis 6jährigen Kindern aus Nordrhein-Westfalen, Modul 2: Glyphosat, 17.03.2016, S. 1, abrufbar unter: www.lanuv.nrw.de (15.06.2019).

53 Antrag der Abgeordneten Christian Lindner u.a., BT-Drs. 19/216 (Fn. 36), S. 3: „... Funde im Urin sind für die Gesundheit nach Aussagen des Bundesamtes für Lebensmittelsicherheit $(B f R)$ für die Gesundheit allerdings obne Bedeutung, da der Wirkstoff unverändert direkt über die Nieren ausgeschieden wird“. 
desamt für Risikobewertung (BfR) allerdings nur für den aus dem Darm resorbierten Anteil des Wirkstoffs bestätigen. ${ }^{54}$ Messbare Befunde von Glyphosat in Muttermilch wurden bislang zwar noch nicht festgestellt. Das BfR geht aber davon aus, dass Glyphosat die biologischen Membrane (Blut-Hirnschranke, Plazentaschranke, BlutMilchdrüsenschranke) wie viele andere Pestizide durchdringen kann. ${ }^{55}$

Soweit es konkrete Gesundheitsrisiken für Lebewesen betrifft, stehen u.a. die Begünstigung der Entstehung bestimmter Krebsarten, Schäden am Erbgut, am hormonellen System, zulasten der Embryonalentwicklung oder des Sehsystems im Fokus der Untersuchungen. ${ }^{56}$ Einen Paukenschlag setzte die zur Weltgesundheitsorganisation gehörende Internationale Agentur für Krebsforschung (IARC), als sie im März 2015 Glyphosat als „wahrscheinlich krebserregend für Menschen (Gruppe 2A)“ einstufte. ${ }^{57}$ Risikogruppe $2 \mathrm{~A}$ bedeutet nach dem Klassifikationssystem der IARC im (hier vorliegenden) Regelfall, dass die Karzinogenität eines Stoffes für Menschen begrenzt, bei Versuchstieren aber hinreichend nachgewiesen werden kann. ${ }^{58}$ Soweit es Menschen betrifft, stellt die Studie einen Zusammenhang zwischen der Glyphosatexposition und dem Non-Hodgkin-Lymphom, einer bösartigen Lymphknotentumorerkrankung, fest, bei Tieren geht es um eher seltenere andere Tumorerkrankungen. Diesen Bewertungen widersprachen im letzten Glyphosat-Genehmigungsverfahren die Europäische Behörde für Lebensmittelsicherheit (European Food Safety Authority [EFSA]) wie auch das mitbeteiligte deutsche Bundesamt für Risikobewertung (BfR), woraufhin es zu einem öffentlichen Schlagabtausch und neuerlichen Bewertungen kam. ${ }^{59}$

In neuerer Zeit ist vermehrt davon die Rede, dass Mittel wie z.B. Glyphosat nur „bei nicht bestimmungsgemäßem Gebrauch“ Risiken begründeten. ${ }^{60}$ Anlass für diese Koppelung der Ungefährlichkeit an den bestimmungsgemäßen Gebrauch dürfte die Entwicklung in Südamerika sein, wo es vor allem in Argentinien infolge eines massenhaften und regelwidrigen Glyphosateinsatzes mithilfe von Sprühflugzeugen zu schwerwiegenden Gesundheitsschädigungen, embryonalen Fehlbildungen und Fehlgeburten gekommen ist, die inzwischen auch zu Verurteilungen der Verantwortlichen

54 Antwort der BReg, BT-Drs. 18/8408 (Fn. 37), S. 17.

55 Antwort der BReg, BT-Drs. 19/4915 (Fn. 15), S. 3.

56 S.u. C I.3. und 4. m.w.N.

57 S. Fn. 43 zu den Ergebnissen der IARC Working Group on the Evaluation of Carcinogenic Risks to Humans (Lyon, 03.-10.03.2015). Abschließende Bewertungen auf S. 398. Dort zum Krebs bei Menschen: "There is limited evidence in humans for the carcinogenicity of glyphosate. A positive association has been observed for non-Hodgkin lymphoma." Dort zum Krebs bei Versuchstieren: "There is sufficient evidence in experimental animals for the carcinogenicity of glyphosate". Dort zur Gesamteinschätzung: "Glyphosate is probably carcinogenic to humans (Group 2A)". Die IARC Monographs sind abrufbar unter: https://mo nographs.iarc.fr (15.06.2019); S.a. den Kurzbericht von Guyton et al., The Lancet Oncology 2015/16, S. 490-491.

58 IARC, (Fn. 43), S. 30.

59 S.u. C I.3. und 4.

60 Siehe etwa Bundesamt für Risikobewertung, Fragen und Antworten zur Bewertung des gesundheitlichen Risikos von Glyphosat, A-Z-Index, FAQ des BfR vom 01. März 2016, abrufbar unter: https://mobil.bfr.bund.de (15.06.2019). 
geführt haben. ${ }^{61}$ Ungeachtet dessen werden glyphosathaltige Mittel aber auch in Staaten mit schwachem Verwaltungsvollzug exportiert und sind meistenteils noch für den privaten Gebrauch zugelassen. So ist z.B. ein glyphosathaltiges Produkt wie „Roundup REKORD“ bis heute (Stand: Januar 2019) in Deutschland durch jede Person, wenn auch in kleinerer Verpackungsgröße, im Handel erhältlich.

Soweit es die Folgen des Gebrauchs glyphosathaltiger Mittel für das Ökosystem und insbesondere die Biodiversität betrifft, wurden ungeachtet der erneuten Genehmigung des Wirkstoffs Glyphosat Risiken gesehen und risikomindernde Auflagen seitens der EU-Mitgliedstaaten angemahnt. ${ }^{62}$ Eine neuere Publikation der Nationalen Akademie der Wissenschaften Leopoldina sieht Problemfelder insbesondere in den unzureichenden Möglichkeiten der Vorhersage der Exposition und der Persistenzbewertung von Chemikalien in der Umwelt, den kaum ermittelbaren Auswirkungen von „Tankmischungen“ aus verschiedenen Pestiziden, Mängeln bei der Bewertung direkter Effekte, einer mangelhaften Berücksichtigung indirekter Effekte sowie dem bislang kaum beachteten Einfluss mehrfacher (multipler) Stressfaktoren. ${ }^{63} \mathrm{Ob}$ und wie genau der unbestreitbar massive Rückgang der Insekten ${ }^{64}$ mit dem Einsatz von Pestiziden oder gar Glyphosat im Besonderen zusammenhängt, ist noch weitgehend unklar und umstritten. Einerseits behaupten Wissenschaftler, dass bei Bienen unter den Bedingungen einer „realistischen worst case-Exposition“ kein erhöhtes Bienensterben festzustellen sei. ${ }^{65}$ Andererseits erscheinen Experimentalstudien, wonach der Navigationssinn von Bienen durch glyphosathaltiges Futter gestört werde. ${ }^{66}$ Die sogenannte „Krefeld-Studie“ (2017) hält die Intensivierung der Landwirtschaft („agricultural intensification") für die einzig plausible Erklärung für den massiven Rückgang der Insekten. ${ }^{67}$ Vortragende eines Internationalen Insektenschutzsymposiums an der Universität Hohenheim forderten in einem viel beachteten Neun-Punkte-Plan ein schrittweises Verbot von Glyphosat innerhalb der nächsten fünf Jahre sowie ein umgehendes vollständiges Verbot in Schutzgebieten, Pufferzonen, Biotopverbundkorridoren und öffentlichen wie privaten Grünflächen, da Totalherbizide wie Glyphosat die Grundlage für artenreiche Nahrungsnetze beseitigten. ${ }^{68}$

Praktisch keine Studie betreffend die Auswirkungen von glyphosathaltigen Mitteln ist aber unumstritten. Regelmäßig werden die Versuchsanordnung, die Repräsentati-

61 S. etwa Burghardt, SZ vom 14.06.2012.

62 S.u. C I.3.b) aa).

63 Schäffer u.a., Nationale Akademie der Wissenschaften - Leopoldina, Halle (Saale), Diskussion Nr. 16, 2018, S. $24 \mathrm{ff}$.

64 S. dazu die Langzeitstudie von Wissenschaftlern des Entomologischen Vereins Krefeld („Krefeld-Studie“), wonach die Gesamt-Biomasse von fliegenden Insekten in geschützten Gebieten zwischen 1989 und 2015 um ca. 75\% zurückgegangen ist: Hallmann et al., PlosOne, 18.10.2017, abrufbar unter: https://doi.org/10.1371/journal.pone.0185809 (15.06.2019).

65 S. etwa Thompson et al., S. 463-470.

66 Balbuena et al., Journal of Experimental Biology 2015/218, S. 2799-2805.

67 Fn. 64.

68 Krogmann u.a., abrufbar unter: www.uni-hohenheim.de (15.06.2019); kritisch dazu: Klein, FAZ Nr. 253 vom 31.10.2018, S. N 1 („Auf diese Tiere können Sie bauen“): zu unspezifisch und nicht weitgehend genug. 
vität der Untersuchungsobjekte, die verwendeten Methoden oder die Bewertung angezweifelt. Die Ursachen für die extrem konträren Positionen auch innerhalb der Wissenschaft sind vielschichtig. Ein Hauptproblem liegt darin, dass Glyphosat nicht als reiner Wirkstoff, sondern in Kombination mit Zusatzstoffen und sogenannten „Netzmitteln“ ausgebracht wird. Bei vergleichbaren anderen Pestiziden ist das Problem ähnlich gelagert. Die wissenschaftlichen Studien sind also schwer vergleichbar, weil unterschiedliche Gegenstände untersucht werden. Die Gefahren können vom eigentlichen Wirkstoff, von Zusatzstoffen des anwendungsfähigen Produkts, Netzmitteln, den Reaktionen miteinander verbundener Stoffe oder auch vom unsachgemäßen Gebrauch ausgehen. Zudem gibt es verschiedene Verfahrensweisen der Untersuchung und unterscheiden sich Art und Intensität der jeweils zugrunde gelegten Glyphosat-Exposition. Besonders schwer zu ermitteln sind die „ökotoxikologischen Kombinationswirkungen" sowie Bioakkumulationen in lebenden Organismen, insbesondere bei Menschen. ${ }^{69}$ Die Wissenschaft geht davon aus, dass ein Stoffgemisch in der Regel eine höhere Toxizität als die einzelnen Komponenten aufweist, ${ }^{70}$ wobei aber im Einzelfall je nach Art, Dosis und Massenverhältnis der Bestandteile der Gemische $^{71}$ (z.B. „Tankmischungen“) die unterschiedlichsten Toxizitätseffekte (additive, potenzierende, synergetische oder umgekehrt auch antagonistische) auftreten können.

\section{B. Zuständigkeiten und Verteilung der Entscheidungsmacht}

Eine zentrale Bedeutung kommt der Frage zu, wer die maßgeblichen Entscheidungen trifft. In der Europäischen Union herrscht im Bereich der Zulassung und Anwendung von Herbiziden und Pestiziden ${ }^{72}$ eine komplexe Zuständigkeitsverteilung mit entsprechend unvermeidlicher „Verantwortungsdiffusion“. Dabei muss grundsätzlich zwischen der Zulassung des Wirkstoffs (z.B. Glyphosat) und der Zulassung von marktfähigen Pflanzenschutzmitteln, die diesen Wirkstoff enthalten (z.B. „Roundup“), unterschieden werden.

69 Siehe statt Vieler Fent, S. 127, 129 (Kombinationswirkungen), $197 \mathrm{ff}$. (Bioakkumulation).

70 Bundesamt für Verbraucherschutz und Lebensmittelsicherheit, Broschüre „Tankmischungen im Zulassungsverfahren für Pflanzenschutzmittel“, November 2015, S. 12 (o.N.).

71 Gemische sind Gegenstand der sogenannten CLP-Verordnung: Verordnung (EG) Nr. 1272/2008 des Europäischen Parlaments und des Rates vom 16. Dezember 2008 über die Einstufung, Kennzeichnung und Verpackung von Stoffen und Gemischen, zur Änderung und Aufhebung der Richtlinien 67/548/EWG und 1999/45/EG und zur Änderung der Verordnung (EG) Nr. 1907/2006 (CLP-Verordnung), ABl. (EG) L 353 v. 31.12.2008, S. 1. Siehe zu den Begriffen „Gemisch“ und „Stoff“ Art. 2 der Verordnung.

72 Unter „Herbizid“ sind Unkrautvernichtungsmittel zu verstehen. Dagegen umfasst der Begriff „Pestizid“ als Oberbegriff Mittel, die entweder auf der Pflanze zum Schutz gegen Befall durch Schädlinge aufgebracht werden (Pflanzenschutzmittel) oder sich direkt gegen Schädlinge wie z.B. Mäuse richten (Biozide etc.). Siehe Europäische Behörde für Lebensmittelsicherheit (EFSA), Stichwort „Pestizide“, abrufbar unter: www.efsa.europa.eu/de/topics/to $\mathrm{pic} /$ pesticides (15.06.2019). 


\section{Genehmigung des Wirkstoffs durch die EU}

\section{Zuständigkeit der EU und Form der Genehmigung: Die Durchführungsverordnung (EU) 2017/2324 zur Erneuerung der Genehmigung des Wirkstoffs Glyphosat}

Für die Zulassung eines Wirkstoffs wie z.B. Glyphosat ist die Europäische Union unter Mitwirkung der Mitgliedstaaten im sogenannten Komitologie-Verfahren zuständig. Im Falle der Zulassung von Herbiziden ergibt sich die Zuständigkeit der EU zur Wirkstoffgenehmigung in erster Linie aus Regelungsbefugnissen der EU zur Schaffung einer gemeinsamen Agrarpolitik und zum Gesundheitsschutz, auf die sich auch die hier einschlägige Verordnung (EG)Nr. 1107/2009 über das Inverkebrbringen von Pflanzenschutzmitteln stützt. ${ }^{73}$ Gemäß Art. 13 Abs. 2 dieser Verordnung erlässt der EU-Gesetzgeber unter Anwendung des in Art. 79 desselben Rechtsakts vorgesehenen Komitologie- oder Ausschussverfahrens eine Verordnung, mit der ein Wirkstoff mit oder ohne Einschränkungen genehmigt wird, nicht genehmigt wird oder die Bedingungen für eine schon bestehende Genehmigung verändert werden. Im Falle von Glyphosat liegt eine solche Genehmigungsverordnung vor, nämlich aktuell die Durchführungsverordnung (EU) 2017/2324 der Kommission zur Erneuerung der Genebmigung des Wirkstoffs Glyphosat gemäß VO (EG) 1107/2009.74 Sie gilt gemäß ihrem Anhang I vom 16. Dezember 2017 bis zum 15. Dezember 2022. Es handelt sich um eine modifizierte erneuerte Genehmigung. Die Erstzulassung von Glyphosat in der damaligen Europäischen Gemeinschaft datiert vom 1. Juli 2002 und wurde bis zum Inkraftreten der Erneuerung mehrfach verlängert. ${ }^{75}$

73 Verordnung (EG) Nr. 1107/2009 des Europäischen Parlaments und des Rates vom 21. Oktober 2009 über das Inverkehrbringen von Pflanzenschutzmitteln und zur Aufhebung der Richtlinien 79/117/EWG und 91/414/EWG des Rates, ABl. (EU) L 309 v. 24.11.2009, S. 1: Die Verordnung verweist im Einzelnen auf Art. 37 Abs. 2 EGV (heute: Art. 43 Abs. 2 AEUV), Art. 95 EGV (heute: Art. 114 AEUV) sowie Art. 152 Abs. 4 lit. b EGV (heute: Art. 168 Abs. 4 lit. b AEUV).

74 Durchführungsverordnung (EU) 2017/2324 der Kommission vom 12. Dezember 2017 zur Erneuerung der Genehmigung des Wirkstoffs Glyphosat gemäß der Verordnung (EG) Nr. 1107/2009 des Europäischen Parlaments und des Rates über das Inverkehrbringen von Pflanzenschutzmitteln und zur Änderung des Anhangs der Durchführungsverordnung (EU) Nr. 540/2011 der Kommission (Text von Bedeutung für den EWR), ABl. (EU) L 333 v. 15.12.2017, S. 10-16.

75 Siehe zu Vorgänger-Richtlinien und -Verordnungen die Hinweise in den Erwägungsgründen zur Durchführungsverordnung (EU) 2017/2324, (Fn. 74): Zu Richtlinie 2001/99/EG v. 20.11.2001 zur Änderung von Anhang I der Richtlinie 91/414/EWG nebst Rechtsakten zur Verlängerung der Laufzeit der Erstgenehmigung Kaus, StoffR 2016, S. 109. 


\section{Einbindung der Mitgliedstaaten im Komitologie-Verfahren}

\section{a) Fachausschuss und Berufungsausschuss für Phytopharmazentika}

Wie erwähnt, wirken die Mitgliedstaaten mithilfe des „Komitologie-Verfahrens“ am Zustandekommen der Durchführungsverordnung der EU-Kommission zur (Neu-)Genehmigung des Wirkstoffs Glyphosat mit. Dabei übt die Kommission ihre Durchführungsbefugnis mit Unterstützung (oder besser Kontrolle) durch Ausschüsse aus, in welchen die Mitgliedstaaten vertreten sind. Je nach Gestaltung der jeweiligen Materie können die Mitgliedstaaten im jeweiligen Ausschuss mehr oder weniger Einfluss auf die Ausführung des EU-Rechts durch die Kommission nehmen. Obwohl für Komitologie-Verfahren gemeinsame allgemeine Regeln existieren ${ }^{76}$ ergeben sich die Einzelheiten für die jeweilige Materie aus dem jeweiligen „Basisrechtsakt“, d.h. hier aus der Pflanzenschutzmittelverordnung (EG) 1107/2009. Danach entscheidet im Falle von Glyphosat zunächst der bei der Kommission angesiedelte Ständige Ausschuss für Pflanzen, Tiere, Lebens- und Futtermittel (Standing Committee on Plants, Animals, Food and Feed [SCoPAFF]), genau genommen der Fachausschuss für Phytopharmazeutica. Dieser Fachausschuss ist mit Experten und Expertinnen aus den EU-Mitgliedstaaten besetzt und wird von der EU-Kommission geleitet. Seine Aufgabe ist es, am Ende eines aufwändigen Bewertungsverfahrens eine Stellungnahme zum Vorschlag der Kommission über eine erneuerte Genehmigungsverordnung abzugeben. ${ }^{77}$ SCoPAFF entscheidet mit qualifizierter Mehrheit, wobei in Anlehnung an die Regeln für Entscheidungen der Mitgliedstaaten im Rat der EU das Prinzip der doppelten Mehrheit gilt: Es müssen mindestens 55\% der Mitgliedstaaten, die mindestens 15 Mitgliedstaaten und 65\% der gesamten EU-Bevölkerung umfassen, dem Verordnungsentwurf zustimmen (Art. 16 Abs. 4 UAbs. 1 EUV)..$^{78}$ Dem entsprechend kann eine Sperrminorität mit 45\% der Mitgliedstaaten plus einem weiteren Mitgliedstaat erreicht werden, sofern diese etwas mehr als 35\% der Bevölkerung aller EUStaaten repräsentieren. Allerdings verschärft Art. 16 Abs. 4 Unterabs. 2 EUV diese Voraussetzungen um das weitere Erfordernis, dass mindestens vier Mitgliedstaaten an der Sperrminorität beteiligt sein müssen.

Kommt im SCoPAFF keine qualifizierte Mehrheit zustande, entscheidet auf Antrag der Kommission ein Berufungsausschuss für den Bereich Phytopharmazeutika (Appeal Committee [ACoPAFF]). Er wird ebenfalls von der Kommission geleitet, ent-

76 Siehe dazu Verordnung (EU) Nr. 182/2011 des Europäischen Parlaments und des Rates vom 16.02.2011 zur Festlegung der allgemeinen Regeln und Grundsätze, nach denen die Mitgliedstaaten die Wahrnehmung der Durchführungsbefugnisse durch die Kommission kontrollieren, ABl. (EU) L 55 v. 28.02.2011, S. 13.

77 Zum Verfahren und zur Bewertung noch u. C.

78 Art. 20 Abs. 1, Art. 79 Abs. 3 VO (EG) 1107/2009 (Basisrechtsakt) i.V.m. Art. 5 Beschluss 1999/468/EG (inzwischen aufgehoben) i.V.m. Art. 13 Abs. 1 lit. c VO (EU) 182/2011 (Überleitungsvorschrift) i.V.m. Art. 5 Abs. 1 VO (EU) 182/2011 i.V.m. Art. 16 Abs. 4 EUV; siehe dazu noch unten C I.3.c). 
scheidet ebenfalls mit qualifizierter Mehrheit, ${ }^{79}$ ist aber anders als der Fachausschuss mit Repräsentanten und Repräsentantinnen der Mitgliedstaaten besetzt.

\section{b) Die Entscheidung vom 27. November 2017 zur Verlängerung der Genehmigung von Glyphosat}

Im Falle der aktuellen Glyphosat-Genehmigungsverordnung war von vornherein klar, dass es sich um eine hoch umstrittene und politisch heikle Entscheidung handeln würde. Am 6. Oktober 2017 hatte die Europäische Bürgerinitiative „Verbot von Glyphosat" eine Liste mit 1,071 Millionen Unterschriften aus 22 Staaten vorgelegt. Es handelte sich um die vierte erfolgreiche europäische Bürgerinitiative überhaupt. ${ }^{80} \mathrm{Am}$ 24. Oktober 2017 legte sich das Europäische Parlament auf ein vollständiges Verbot von Herbiziden auf Glyphosatbasis bis 2022 fest. $^{81}$

Nachdem im Fachausschuss für Phytopharmazeutika keine qualifizierte Mehrheit für den Vorschlag der Kommission über eine erneuerte Genehmigungsverordnung erreicht werden konnte, erarbeitete die Kommission einen neuen Textentwurf und rief den Berufungsausschuss an. ${ }^{82}$ Bei dessen Entscheidung am 27. November 2017 kam die qualifizierte Mehrheit nur denkbar knapp zustande, nämlich mit 18 Jastimmen und 9 Neinstimmen bei einer Enthaltung, wobei für das Erreichen der doppelten Mehrheit ausschlaggebend war, dass der Bundesminister für Ernährung und Landwirtschaft Christian Schmidt (CSU) den deutschen Vertreter im ACoPAFF letztlich per Mail anwies, mit „Ja“ zu stimmen. Dem deutschen Vertreter vor Ort sei dabei die Haltung aller beteiligter Ressorts bekannt gewesen, d.h. die Uneinigkeit innerhalb der Bundesregierung und der unmissverständliche Widerspruch der Umweltministerin Hendricks (SPD); die Bundeskanzlerin habe aber „erst mit der Abstimmung vom Abstimmungsverhalten des Bundesministers erfahren" ${ }^{83}$ Der zwischen den Ministerien abgestimmte Sprachzettel des deutschen Vertreters hatte noch die Weisung enthalten, sich zu enthalten. ${ }^{84}$

79 Art. 20 Abs. 1, Art. 79 Abs. 3 VO (EG) 1107/2009 (Basisrechtsakt) i.V.m. Art. 5 Beschluss 1999/468/EG (inzwischen aufgehoben) i.V.m. Art. 13 Abs. 1 lit. c VO (EU) 182/2011 (Überleitungsvorschrift) i.V.m. Art. 6 Abs. 1 VO (EU) 182/2011 i.V.m. Art. 5 Abs. 1 VO (EU) 182/2011 i.V.m. Art. 16 Abs. 4 EUV;siehe dazu noch unten C I.3.c).

80 Verbot von Glyphosat und Schutz von Menschen und Umwelt vor giftigen Pestiziden, Registriernummer der Kommission: ECI(2017)000002, Datum der Registrierung: 25/01/2017; zu den Konsequenzen der Bürgerinitiative noch u. C I.2.c).

81 S.u. B I.3.

82 S.u. C I.3.a).

83 BT-Drs. 19/280 vom 15.12.2017, S. 48, 50.

84 Antwort der Bundesregierung auf die Kleine Anfrage der Abgeordneten Dr. Marco Buschmann u.a. und der Fraktion der FDP, BT-Drs. 19/286, S. 2. 
Dieses Abstimmungsverhalten widersprach der im Koalitionsvertrag vorgegebenen Marschrichtung zum Thema Glyphosat, ${ }^{85}$ der Vorgabe, ein „geschlossenes Auftreten“ der Koalition gegenüber den europäischen Institutionen sicherzustellen und sich unter Beibehaltung der bewährten Zuständigkeitsverteilung innerhalb der Regierung „vorher eng ab[zu]stimmen“", 86 wie auch der Geschäftsordnung der Bundesregierung. ${ }^{87}$ Trotzdem besteht kein Zweifel daran, dass die Erklärung des deutschen Vertreters in Brüssel im Außenverhältnis (gegenüber der EU) wirksam geworden ist. Ob die Erklärung des Ministers zutrifft, er habe nur durch seine Zustimmung erreichen können, den Glyphosat-Einsatz „für privaten Gebrauch und für andere Gebräuche zu reduzieren “88 und es seien „wichtige Verbesserungen, zum Beispiel für die Pflanzen- und Tierwelt, durchgesetzt worden", 89 wird noch an anderer Stelle zu vertiefen sein. ${ }^{90}$

\section{Die Rolle des Europäischen Parlaments}

Die Wirkstoffgenehmigung liegt, wie gezeigt, im europäischen Bereich auf Seiten der Exekutive, d.h. der Kommission, die über die maßgebliche Durchführungsverordnung im Komitologieverfahren unter Mitwirkung der Regierungen der Mitgliedstaaten entscheidet. Dem entsprechend schwach ausgeprägt sind die Möglichkeiten des Europäischen Parlaments, auf die Genehmigungsentscheidung Einfluss zu nehmen. Soweit es die konkrete Wirkstoffgenehmigung (Erlass der Durchführungsverordnung) betrifft, beschränkt sich die Rolle des Parlaments auf relativ umfassende Informationsrechte in Bezug auf den Ablauf und die Ergebnisse des Ausschussverfahrens sowie das Recht, mögliche Kompetenzüberschreitungen beim Gebrauch der Durchführungsbefugnisse zu beanstanden (Kontrollrecht). ${ }^{91}$ Das hat das EP im Glyphosat-

85 Siehe Koalitionsvertrag (19. Legislaturperiode) vom 12.03.2018, S. 142 (Biodiversitätsschutz): „Wir werden mit einer systematischen Minderungsstrategie den Einsatz von glyphosathaltigen Pflanzenschutzmitteln deutlich einschränken mit dem Ziel, die Anwendung so schnell wie möglich grundsätzlich zu beenden. Dazu werden wir gemeinsam mit der Landwirtschaft Alternativen im Rabmen einer Ackerbaustrategie entwickeln und u.a. umwelt- und naturverträgliche Anwendungen von Pflanzenschutzmitteln regeln. Die dazu notwendigen rechtlichen Maßnabmen werden wir in einem EU-konformen Rabmen verankern.", abrufbar auf den Seiten der Regierungsparteien CDU, CSU und SPD, z.B. unter: www.cdu.de/system/tdf/media/dokumente/koalitionsvertrag_2018.pdf?file=1 (15.06.2019).

86 Ibid., S. 174.

87 So auch die Auffassung der Bundeskanzlerin in diesem Fall; siehe BT-Drs. 19/286 (Fn. 84), S. 1: Es geht dabei um Art. $16 \mathrm{f}$. der Geschäftsordnung der Bundesregierung betreffend die Beilegung von Meinungsverschiedenheiten im Kabinett; siehe ferner $\$ 19$ Abs. 1 der Gemeinsamen Geschäftsordnung der Bundesministerien (GGO) vom 01.09.2011.

88 Zeit online vom 28.11.2017 (Glyphosat: CSU Minister Schmidt traf Entscheidung „für sich“), abrufbar unter: www.zeit.de (15.06.2019).

89 BT-Drs. 19/286, (Fn. 84), S. 2.

90 S.u. C I.3.

91 Art. 20 Abs. 1, Art. 79 Abs. 3 VO [EG] 1107/2009 (Basisrechtsakt) i.V.m. Art. 7, 8 Beschluss 1999/468/EG (inzwischen aufgehoben) i.V.m. Art. 13 Abs. 1 lit. c VO (EU) 182/2011 (Überleitungsvorschrift) i.V.m. Art. 10 f. VO (EU) 182/2011; siehe dazu noch unten C I.3.c). 
Fall auch tatsächlich getan, jedoch ohne nennenswerte Folgen. ${ }^{92}$ Darüber hinaus konnte das Parlament beim Zustandekommen des „Basisrechtsakts“, d.h. der europäischen Pflanzenschutzmittelverordnung (EG) 1107/2009, im Gesetzgebungsverfahren eine gewisse Rolle spielen und wird sie bei Änderungen dieses Rechtsakts spielen können, wird insoweit aber durch das Vorschlagsrecht der Kommission beschränkt (Art. 289 Abs. 1, Art. 294 Abs. 2 AEUV).

Unbenommen bleibt dem Parlament nur, sich in Form von Entschließungsbeschlüssen politisch zu äußern, wie es gerade im Falle der Erneuerung der GlyphosatWirkstoffgenehmigung geschehen ist. Am 24. Oktober 2017 verabschiedete das Europäische Parlament in Bezug auf die damals anstehende Erneuerung der GlyphosatWirkstoffgenehmigung eine Entschließung, die ein vollständiges Verbot von Herbiziden auf Glyphosatbasis bis 2022 forderte. ${ }^{93}$ Damit änderte es seine noch im Frühjahr 2016 vertretene Position, als es sich trotz schwerwiegender Bedenken unter der Voraussetzung massiver Beschränkungen noch für eine Wiedergenehmigung für sieben Jahre ausgesprochen hatte. ${ }^{94}$

Nach der Kontroverse über die erneute Zulassung von Glyphosat hat das Europäische Parlament mit Beschluss vom 6. Februar 2018 einen Sonderausschuss für das Genehmigungsverfahren der EU für Pestizide (PEST) eingesetzt, der die Aufgabe erhielt, das Genehmigungsverfahren der EU für Pestizide umfassend zu untersuchen. Im Abschlussbericht stellte der Sonderausschuss schwerwiegende Mängel bei der $\mathrm{Zu}$ lassung von Pestiziden fest, woraufhin das Parlament per Entschließung vom 16. Januar 2019 eine grundlegende Reform des Zulassungsverfahrens für Pestizide forderte, insbesondere soweit es dessen Transparenz und die Unabhängigkeit der zugrunde liegenden Forschung betrifft. ${ }^{95}$

92 Ziffer 1 der Entschließung des Europäischen Parlaments zu dem Entwurf einer Durchführungsverordnung der Kommission zur Erneuerung der Genehmigung des Wirkstoffs Glyphosat gemäß der Verordnung (EG) Nr.1107/2009, 2017/2904(RSP) vom 19.10.2017, D053565-01 - 2017/2904(RSP), P8_TA(2017)0395.

93 Entschließung des Europäischen Parlaments, (Fn. 92).

94 Ziffer 3 der Entschließung des Europäischen Parlaments vom 13. April 2016 zu dem Entwurf einer Durchführungsverordnung der Kommission zur Erneuerung der Genehmigung des Wirkstoffs Glyphosat gemäß der Verordnung (EG) Nr. 1107/2009 des Europäischen Parlaments und des Rates über das Inverkehrbringen von Pflanzenschutzmitteln und zur Änderung des Anhangs der Durchführungsverordnung (EU) Nr. 540/2011, D044281-01 2016/2624(RSP), P8_TA(2016)0119.

95 Abschlussbericht des PEST-Ausschusses vom 18.12.2018: Special Committee on the Union's authorisation procedure for pesticides, Report on the Union's authorisation procedure for pesticides, A8-0475/2018, 2018/2153(INI); Annahme der Empfehlungen durch das EP: European Parliament resolution of 16 January 2019 on the Union's authorisation procedure for pesticides, P8_TA(2019)0023, 2018/2153(INI), abrufbar unter: www.europarl.europa.eu (15.06.2019). 


\section{Zulassung glyphosathaltiger Pflanzenschutzmittel durch die Mitgliedstaaten am Beispiel Deutschlands}

Die Zulassung glyphosathaltiger Pflanzenschutzmittel liegt in der Hand der Mitgliedstaaten, soweit und solange eine Genehmigung des Wirkstoffs Glyphosat auf EU-Ebene besteht. Gemäß Art. 28-54 der Verordnung (EG) 1107/2009 entscheiden die Mitgliedstaaten unter Berücksichtigung ihrer jeweiligen klimatischen und landwirtschaftlichen Bedingungen. ${ }^{96}$ Innerhalb des Mitgliedstaats Deutschland ist die $\mathrm{Zu}$ ständigkeit für die Zulassung von Pflanzenschutzmitteln wiederum in Entscheidungskompetenzen des Bundes und der Länder unterteilt. Für generelle Fragen der Zulassung von Pflanzenschutzmitteln einschließlich der Risikobewertung ist nach Art. 74 Abs. 1 Nr. 20 GG der Bund zuständig, für Einzelfallgenehmigungen und die Überwachung der Anwendung sind gemäß Art. 83 GG die Bundesländer zuständig. Sie unterliegen dabei keinerlei Weisungen von Seiten der Bundesbehörden.

\section{Entscheidungsstrukturen auf Bundesebene}

Für die Zulassung von Pflanzenschutzmitteln ist in Deutschland gemäß $\$ 33$ Abs. 1 PflSchutzG das Bundesamt für Verbraucherschutz und Lebensmittelsicherheit $(B V L)$ zuständig. Genau genommen handelt es sich um eine ganze Reihe von Zuständigkeiten, u.a. für die (Erst-)Zulassung eines Pflanzenschutzmittels nach Art. 29 der Verordnung (EG) 1107/2009, die Erneuerung der Zulassung eines Pflanzenschutzmittels nach Art. 43 VO (EG) 1107/2009 oder die Aufhebung oder Änderung der Zulassung eines Pflanzenschutzmittels nach Art. $44 \mathrm{f}$. VO (EG) 1107/2009. Außerdem hat das BVL gemäß $\ 58$ PflSchutzG Überwachungsfunktionen, insbesondere wirkt es an der Überwachung zugelassener Pflanzenschutzmittel mit ( $\$ 58$ Abs. 1 Nr. 2 PflSchutzG).

Das BVL ist eine selbstständige Bundesoberbehörde im Geschäftsbereich des Bundesministeriums für Ernährung und Landwirtschaft (BMEL). Als selbstständige Bundesoberbehörde (Art. 87 Abs. 3 GG) hat es keinen eigenen Verwaltungsunterbau (Mittel- und Unterbehörden) und keine Rechtsfähigkeit. Es genießt zwar eine gewisse Verselbständigung nach außen (organisatorische Ausgliederung, „Stempelrecht“), ist aber keineswegs "ministerialfrei“, sondern agiert im Geschäftsbereich und unter dem Einfluss des BMEL. Allerdings befindet es über die Zulassung von Pflanzenschutzmitteln nicht allein, sondern muss andere Behörden einbinden. Gemäß Art. 34 Abs. 1 Nrn. 1-3 PflSchutzG entscheidet das BVL

- im Benehmen mit dem Bundesinstitut für Risikobewertung $(B f R)^{97}$ hinsichtlich der Gesundheit von Mensch und Tier, der Vermeidung gesundheitlicher Schäden durch Belastung des Bodens sowie hinsichtlich der Analysemethoden für Rückstände gemäß Art. 29 Abs. 1 Buchst. g VO (EG) 1107/2009, 
- im Benehmen mit dem Julius Kühn-Institut ${ }^{98}$ hinsichtlich der Wirksamkeit, unvertretbarer Auswirkungen auf die zu schützenden Pflanzen, Pflanzenerzeugnisse und Honigbienen sowie hinsichtlich vermeidbarer Leiden und Schmerzen bei Wirbeltieren, zu deren Bekämpfung das Pflanzenschutzmittel vorgesehen ist, und

- im Einvernehmen mit dem Umweltbundesamt $(U B A)$ hinsichtlich der Vermeidung von Schäden durch Belastung des Naturhaushaltes sowie durch Abfälle des Pflanzenschutzmittels.

Das bedeutet, dass das Bundesamt für Verbraucherschutz und Lebensmittelsicherheit (BVL) das Bundesinstitut für Risikobewertung (BfR) und das Julius Kühn-Institut auf den jeweils genannten Feldern einschalten, sich mit ihnen austauschen und deren Expertise nutzen muss („Benehmen“). Dagegen muss, soweit es um die Vermeidung von Umweltschäden geht, „Einvernehmen“ mit dem Umweltbundesamt hergestellt werden. In Bezug auf das erforderliche Benehmen mit dem BfR ist von Interesse, dass das BfR zwar anders als das BVL eine rechtsfähige Bundesbehörde ( $\$ 1$ BfRG) ist, beide Bundesämter aber im Geschäftsbereich des Bundesministeriums für Ernährung und Landwirtschaft (BMEL) liegen. Obwohl aufgrund des jeweils unterschiedlichen Grades an rechtlicher Verselbständigung die Möglichkeiten der Einflussnahme des BMEL gegenüber dem BVL größer als gegenüber dem BfR sind, bleibt die Frage kritisch, ob es eine ausreichende Trennung zwischen der Risikobewertung durch das BfR und dem Risikomanagement (Zulassung von Pflanzenschutzmitteln) durch das BVL gibt. Allein die Tatsache, dass das BfR nicht für die Zulassung zuständig ist, ${ }^{99}$ genügt hierfür nicht.

Ganz anders verhält es sich im Falle des erforderlichen Einvernehmens mit dem Umweltbundesamt (UBA). Denn das UBA ist als selbstständige Bundesoberbehörde (Art. 87 Abs. 3 GG) im Geschäftsbereich des Bundesministeriums für Umwelt, Naturschutz und Reaktorsicherheit (BUM) angesiedelt. Es hat damit eine vergleichbare Rechtsstellung wie das beim BMEL angesiedelte BVL. Werden die beiden „im Hintergrund stehenden" Bundesministerien (BMEL und BUM) im Falle einer Koalitionsregierung auf Bundesebene von Personen unterschiedlicher Parteizugehörigkeit geführt, kann es zu Differenzen beim Zusammenspiel der beiden Bundesämter kommen. Das BMEL wird seit November 2005 durchgehend von einem CSU-Bundesminister bzw. einer CSU-Bundesministerin geführt, die eher wenig Neigung verspürten, den Herbizid-Verbrauch in der Landwirtschaft einzuschränken. Dem gegenüber wird das BUM seit Dezember 2013 von SPD-Ministerinnen geführt, die sich zunehmend klar für den schrittweisen Ausstieg aus der Glyphosat-Verwendung und gegen

98 Siehe $\$ 58$ PflSchG.

99 So aber die Argumentation der Bundesregierung, BT-Drs. 18/8408, S. 5 (zu Frage 13). 
die Verwendung vergleichbarer Ersatzmittel positionierten, ${ }^{100}$ so dass Konflikte in einer Großen Koalition zwischen diesen Beteiligten vorprogrammiert waren. Die grundlegenden politischen Meinungsverschiedenheiten betreffen dabei nicht nur die innerstaatliche Zulassung von glyphosathaltigen Mitteln in Deutschland, sondern auch Entscheidungen auf der europäischen Ebene wie die Erneuerung der europäischen Genehmigungsverordnung für Glyphosat im November 2017. Ein wichtiger Unterschied liegt allerdings darin, dass das BUM bei der Zulassung von glyphosathaltigen Pflanzenschutzmitteln seine Zustimmung erteilen muss (Art. 34 Abs. 1 Nr. 3 PflSchutzG), während sich bei der Zulassung des Wirkstoffs auf der europäischen Ebene das BMEL notfalls über das BMU hinwegsetzen kann. Denn in diesem Verhältnis geben nur Koalitionsvertrag und Geschäftsordnungsregeln die Abstimmung zwischen den Bundesministerien vor, ${ }^{101}$ mit der Folge, dass bei fehlender Abstimmung allenfalls ein politischer Preis zu zahlen ist.

\section{Entscheidungsstrukturen auf Länderebene und Verzahnung mit Bundesinstitutionen}

Wie sich aus $\ 59$ Abs. 1 PflSchG ergibt, obliegen den nach Landesrecht zuständigen Behörden der Bundesländer nahezu alle Durchführungs- und Kontrollaufgaben, namentlich

- die Durchführung des Pflanzenschutzgesetzes und die Überwachung der Einhaltung seiner Vorschriften,

- die nach Art. 68 VO (EG) 1107/2009 vorgesehenen Kontrollen,

- die Durchführung des Aktionsplanes nach $\$ 4$ PflSchG,

- die Durchführung der nach dem Pflanzenschutzgesetz vorgesehenen Verordnungen wie z.B. der für die Einschränkung des Glyphosatgebrauchs relevanten Pflanzenschutz-Anwendungsverordnung und

- die Durchführung der nach dem PflSchG erteilten Auflagen.

100 Siehe zum Ausstiegsplan der Bundesumweltministerin Svenja Schulze (SPD) die Pressemitteilung des BMU vom 06.11.2018 auf der Homepage des BUM, abrufbar unter: www.bmu.de/pressemitteilung/schulze-legt-plan-fuer-glyphosat-ausstieg-vor/ (13.12.2018); in ihrer Stellungnahme vom 27.09.2018, Homepage ibid., erläutert sie, warum eine Fokussierung auf Glyphosat dabei zu kurz greift: „Wir wollen einen grundsätzlichen Ausstieg aus der Glyphosat-Nutzung bis zum Ende dieser Legislaturperiode. Doch dabei darf es nicht bleiben. Glyphosat ist zwar ein mengen-und flächenmäßig besonders bedeutendes Pflanzenschutzmittel-aber es ist eben nur eines von vielen. Daber greift ein Ansatz, der rein auf die Reduzierung von Glyphosat setzt, zu kurz: Wenn Glyphosat erst einmal weg ist, wird diese Lücke womöglich mit einem ähnlich bedenklichen oder noch bedenklicheren anderen Pflanzenschutzmittel ausgefüllt. Das kann nicht die Lösung sein. Generell soll die Anwendung von Pflanzenschutzmitteln umwelt-und naturverträglich ausgestaltet werden, nicht zuletzt durch eine deutlich stärkere Berücksichtigung der biologischen Vielfalt bei der Zulassungspraxis und den Anwendungsbestimmungen für Pflanzenschutzmittel. Die Grundlage dafür liefert uns der Koalitionsvertrag. .... ".

101 S.o. B I.2.b). 
Dabei führen die Pflanzenschutzdienste der Bundesländer insbesondere „Verkehrskontrollen" (Sachkunde und Beratung in Verkaufsstellen, Verkehrsfähigkeit und korrekte Bezeichnung der Produkte) und „Anwendungskontrollen“ (Anwendung nur zugelassener Mittel gemäß Anwendungsbestimmungen) durch. Die Lebensmittelund Futtermittelüberwachung in den Bundesländern analysiert Lebensmittel und Futtermittel auf Rückstände von Pflanzenschutzmitteln. All diese Informationen laufen, wie sogleich gezeigt wird, beim BVL als nationale Kontakt- und Koordinierungsstelle zusammen. Dagegen koordiniert das BUM die Erkenntnisse aus den Bundesländern zu Pflanzenschutzmittelrückständen im Grund-, Oberflächen- und Trinkwasser.

Soweit es den eigentlichen Pflanzenschutz betrifft, wurde nicht zuletzt mit Blick auf die europarechtlichen Anforderungen erkannt, dass gemeinsame Standards für ganz Deutschland vonnöten sind. Zu diesem Zweck haben Bund und Länder ein gemeinsames „Pflanzenschutz-Kontrollprogramm“ ins Leben gerufen. ${ }^{102} \mathrm{Im}$ Rahmen dieses Programms arbeitet die Arbeitsgemeinschaft Pflanzenschutzmittelkontrolle (AG PMK) mit Fachleuten der Länder unter der Geschäftsführung des BVL Empfehlungen für gemeinsame Standards in Form eines Handbuchs aus und koordiniert das Kontrollprogramm. Die Bundesländer melden ihre Kontrollergebnisse dem BVL, das sie gegebenenfalls in das europäische Schnellwarnsystem für Lebens- und Futtermittel einspeist, in Jahresberichten auswertet und ein Labor zur Untersuchung von Pflanzenschutzmittelproben zur Verfügung stellt. Sinn der Jahresberichte ist es, Vollzugsdefizite zu erkennen, um Empfehlungen für nötige Korrekturen auch im Bereich der Gesetzgebung geben zu können. All dies erscheint vernünftig organisiert. Sieht man sich die Kontrollberichte allerdings näher an, zeigen sich die typischen Probleme jeder Form von Bund-Länder-Zusammenarbeit. ${ }^{103}$ Es gelingt zwar nur so, gemeinsame Standards zu etablieren und unionsrechtlichen Vorgaben im ganzen Bundesgebiet gleichmäßig und vergleichbar Rechnung zu tragen. Unterschiedliche Positionen auf Seiten des Bundes und der einzelnen Länder begünstigen aber Einigungen auf dem kleinsten gemeinsamen Nenner - was keine Option in puncto Kontrolle und Rechtsdurchsetzung ist.

\section{Genehmigungsverfahren und Risikobewertung}

\section{EU-Wirkstoffzulassung}

\section{Ablauf des Genehmigungsverfahrens und Mechanismus der Risikobewertung}

Auf der europäischen Ebene vollzieht sich die Wirkstoffgenehmigung in einem komplexen Verfahren mit zahlreichen Akteuren. Für Erneuerungsverordnungen zur Wiedergenehmigung eines schon früher zugelassenen Wirkstoffs (wie z.B. Glyphosat) gilt

102 Siehe auch zum Folgenden die Homepage des BVL (www.bvl.bund.de [15.06.2019]) unter Pflanzenschutzmittel/Aufgaben/Pflanzenschutz-Kontrollprogramm.

103 S.u. D II.3. 
gemäß Art. 20 Abs. 1 VO (EG) 1107/2009 das Ausschuss- oder Komitologieverfahren gemäß Art. 79 Abs. 3 VO (EG) 1107/2009. Diese Norm verweist auf die Artikel 5 und 7 des inzwischen aufgehobenen Beschlusses 1999/468/EG. Was heute anstelle dieser Artikel gilt, ist in einer Übergangsbestimmung der neuen Komitologie-Verordnung (EU) 182/2011 104 ausgeführt: Gemäß Art. 13 Abs. 1 lit. c VO (EU) 182/2011 findet im Falle der Verweisung auf Art. 5 des obsoleten Beschlusses 1999/468/EG das in Art. 5 VO (EU) 182/2011 genannte Prüfverfahren Anwendung. Im Prüfverfahren kann sich die Kommission - anders als im Beratungsverfahren - grundsätzlich nicht über eine Ablehnung seitens der Mitgliedstaaten im Ausschuss hinwegsetzen, sofern diese eine qualifizierte Mehrheit ${ }^{105}$ gegen den Kommissionsvorschlag zustande bringen. Die Kommission ist in diesem Verfahren also stärker durch die Mitgliedstaaten „kontrolliert“.

Obwohl das Verfahren zur Erneuerung einer zuvor schon bestehenden Genehmigung wie im Falle von Glyphosat im Großen und Ganzen wie bei der Erstgenehmigung verläuft, muss berücksichtigt werden, dass es Spezialregelungen für das Erneuerungsverfahren gibt. Dazu zählt im Falle von Glyphosat die Durchführungsverordnung (EU) Nr. 1141/2010 der Kommission. ${ }^{106}$ Zudem haben sich die einschlägigen Rechtsakte seit der Erstzulassung von Glyphosat verändert. Folgende Etappen werden im (Wieder-)Genehmigungsverfahren durchlaufen: ${ }^{107}$

1. Das antragstellende Unternehmen (hier: das Unternehmenskonsortium “Glyphosate Task Force“) stellt den Genehmigungsantrag beim sogenannten „berichterstattenden Mitgliedstaat“ (Rapporteur Member State - RMS), ${ }^{108}$ dem zwei Dossiers (Volltext und Kurzfassung) mit umfangreichen Datenanforderungen beigefügt sein müssen. ${ }^{109}$ Bei Erstanträgen wählt die Antragstellerin den berichterstattenden Mitgliedstaat, bei Erneuerungsanträgen bestimmt ihn die Kommission. Im Falle der letzten Glyphosatgenehmigung war dies Deutschland. ${ }^{110}$

104 S.o. Fn. 76.

105 S.o. B I.2.a).

106 Art. 14-20 VO (EG) 1107/2009; Verordnung (EU) Nr. 1141/2010 der Kommission vom 07.12.2010 zur Festlegung des Verfahrens für die erneute Aufnahme einer zweiten Gruppe von Wirkstoffen in Anhang I der Richtlinie 91/414/EWG des Rates und zur Erstellung der Liste dieser Wirkstoffe.

107 S. zum folgenden auch die ausführliche Darstellung im Schlussantrag der Generalanwältin Sharpston vom 12.03.2019, EuGH, Rs. C-616/17, Procureur de la République gegen Mathieu Blaise et al., ECLI:EU:C:2019:190, Rn. $13 \mathrm{ff}$.

108 Art. 35 ff. VO (EG) 1107/2009.

109 Art. 7 i.V.m. Nr. 3 des Anhangs II zur VO (EG) 1107/2009; sowie Art. 8 Abs. 1 lit. b VO (EG) 1107/2009 i.V.m. Verordnung (EU) Nr. 283/2013 der Kommission vom 1. März 2013 zur Festlegung der Datenanforderungen für Wirkstoffe gemäß der Verordnung (EG) Nr. 1107/2009, ABl.(EU) L 93 v. 03.04.2013, S.1; und Art. 8 Abs. 1 lit. c VO (EG) 1107/2009 i.V.m. Verordnung (EU) Nr. 284/2013 der Kommission vom 1. März 2013 zur Festlegung der Datenanforderungen für Pflanzenschutzmittel gemäß der Verordnung (EG) Nr. 1107/2009 des Europäischen Parlaments und des Rates über das Inverkehrbringen von Pflanzenschutzmitteln, ABl. (EU) L 93 v. 03.04.2013, S. 85.

110 Art. 7 Abs. 1 VO (EG) 1107/2009, zu Deutschlands Rolle als RMS bei der GlyphosatNeugenehmigung u. C I.3.a). 
2. Der RMS prüft die Zulässigkeit des Antrags, informiert die Beteiligten und beginnt mit der Bewertung. ${ }^{111}$

3. Der RMS erstellt den Entwurf eines Bewertungsberichts (Draft Assessment Report), die sogenannte „unabhängige, objektive und transparente Bewertung“. Bei Genehmigungserneuerungen ist vom "Renewal Assessment Report $(R A R)$ “ die Rede. Zentraler Inhalt ist dabei die Risikobewertung, d.h. die „voraussichtliche“ Erfüllung der Kriterien des Art. 4 VO [EG] 1107/2009, nach dem neuesten Stand von Wissenschaft und Technik. ${ }^{112}$

4. Peer-Review (Art. 12 VO [EG] 1107/2009): Der berichterstattende Mitgliedstaat leitet den Bewertungsberichtsentwurf an die Europäische Kommission und die Behörde für Lebensmittelsicherheit (EFSA), die ihn wiederum den anderen Mitgliedstaaten weiterleitet. ${ }^{113}$ Die EFSA überprüft den Bewertungsbericht des RMS, verlangt gegebenenfalls Aktualisierung von der Antragstellerin, führt Konsultationen mit Sachverständigen durch und informiert die Öffentlichkeit, wobei die Antragstellerin die Vertraulichkeit bestimmter Teile des Bewertungsberichtsentwurfs beantragen kann. Gegebenenfalls werden zusätzliche Informationen beigezogen. In einem „Peer Review Report“ werden die Materialien zusammengestellt und veröffentlicht, insbesondere die Rückmeldungen der Mitgliedstaaten und die Ergebnisse der Konsultationen.

5. Auf der Basis des gegebenenfalls überarbeiteten Bewertungsberichtsentwurfs des RMS erstellt die EFSA einen europäischen Bewertungsbericht, die EFSA-Schlussfolgerung (EFSA Conclusions). ${ }^{114}$

6. Unter Berücksichtigung der Bewertung durch den RMS, eventueller Stellungnahmen (der anderen Mitgliedstaaten oder Antragstellerin) sowie der EFSA-Schlussfolgerung erarbeitet die Europäische Kommission einen „Überprüfungsbericht“ bzw. „Beurteilungsbericht“115 und einen Entscheidungsvorschlag, d.h. im positiven Falle den Entwurf einer befristeten Durchführungsverordnung zur (erneuerten) Genehmigung des betreffenden Wirkstoffs (Art. 13 VO [EG] 1107/2009). Sie gibt dem Antragsteller Gelegenheit zur Stellungnahme und leitet das KomitologieVerfahren (7) ein. ${ }^{116}$

7. Komitologie-Verfahren: ${ }^{117}$ Der Ständige Ausschuss für Pflanzen, Tiere, Lebensund Futtermittel (EU Standing Committee on Plants, Animals, Food and Feed [SCoPAFF]) entscheidet mit qualifizierter Mehrheit über seine Stellungnahme zum Verordnungsentwurf der Kommission. Kann sich der SCoPAFF nicht in-

111 S. i.E. Art. 9 Abs. 3 VO (EG) 1107/2009.

112 Art. 11 VO (EG) 1107/2009.

113 S. i.E. Art. 11 Abs. 1, Art. 12 Abs. 1 VO (EG) 1107/2009.

114 Art. 12 Abs. 2 VO (EG) 1107/2009.

115 Dem englischen Begriff „Review Report“ entspricht „Überprüfungsbericht“ in Art. 13 VO [EG] 1107/2009, aber „Beurteilungsbericht“ in Art. 17 VO (EG) 1141/2010: Der Bericht begleitet den Entscheidungsvorschlag (DurchführungsVO zur Genehmigung) und wird mit diesem dem Ausschuss vorgelegt. Im weiteren Verlauf unterstützt er die Mitgliedstaaten bei deren Entscheidungen über die (Wieder-)Zulassung von Anwendungsprodukten.

116 Art. 13 Abs. 2 VO (EG) 1107/2009.

117 Art. 13 Abs. 2, Art. 79 Abs. 3 VO (EG) 1107/2009. S. zu den Detailregelungen B I.2.a). 
nerhalb der Frist mit qualifizierter Mehrheit auf eine Stellungnahme einigen, kann der Vorsitz des Ausschusses (die Kommission) den Berufungsausschuss (ACoPAFF) anrufen.

8. Im Rahmen des Ausschussverfahrens wird der Überprüfungs- bzw. Beurteilungsbericht finalisiert und gegebenenfalls auch der Verordnungsentwurf zur Neugenehmigung noch einmal verändert. Hat der (Berufungs-)Ausschuss eine positive Stellungnahme abgegeben, verabschiedet die Kommission eine Durchführungsverordnung, mit der sie die Genehmigung des Wirkstoffs entsprechend der Stellungnahme bestätigt oder ablehnt. ${ }^{118}$ Der (wieder-)genehmigte Wirkstoff wird anschließend in den Anhang der DurchführungsVO (EU) 540/2011 aufgenommen, wo alle in der EU genehmigten Wirkstoffe gelistet sind. ${ }^{119}$

9. Im Falle der Erneuerung der Genehmigung für den Wirkstoff durch eine neue Durchführungsverordnung müssen die Mitgliedstaaten alle zugelassenen Pflanzenschutzmittel neu bewerten. ${ }^{120}$

Eine Besonderheit dieses Verfahrens liegt darin, dass die Unternehmen mit eigenen wissenschaftlichen Studien vorklären, ob die Kriterien des Art. 4 VO (EG) 1107/2009 erfüllt sind. Sie sind nämlich verpflichtet, in ihren Antragsdossiers (Schritt 1) die sogenannten „Originalstudien“ über die Wirksamkeit und potentiellen Gefahren ihres Wirkstoffes und mindestens eines Anwendungsmittels vorzulegen (Art. 8 VO [EG] 1107/2009). Trifft dieser Mechanismus mit einem großzügigen Schutz der Betriebsund Geschäftsgeheimnisse zusammen und sind die nachfolgend bewertenden Behörden personell und organisatorisch zu schwach aufgestellt, um die geforderte „unabhängige, objektive und transparente Bewertung" tatsächlich zu leisten, oder gar beeinflusst, sind die Folgen vorprogrammiert: Das interessierte Unternehmen(skonsortium) bestätigt selbst die Unbedenklichkeit seiner Produkte. ${ }^{121}$

\section{Maßstäbe der Risikobewertung}

Zentral für die Risikobewertung ist u.a. der Begriff des „Risikos“. Interessanterweise finden sich in den zahlreichen Legaldefinitionen in Art. 3 der Pflanzenschutz-Verordnung (EG) 1107/2009 keine Hinweise auf „Risiko“, „Gefahr“ und dergleichen. Solche Definitionen enthält aber die Lebensmittelrecht-Verordnung (EG)

118 Art. 20 VO (EG) 1107/2009 zur Frage, ob die Kommission auch ohne positive Stellungnahme genehmigen kann, noch u. C I.3.c).

119 Art. 13 Abs. 2 und 4, Art. 78 Abs. 3 VO (EG) 1107/2009; gemäß Art. 13 Abs. 4 VO [EG] 1107/2009 i.V.m. Art. 2 DurchführungsVO (EU) 2017/2324 wird der Anhang der DurchführungsVO (EU) 540/2011 entsprechend der neuen Genehmigung geändert; s. für Erneuerungsverordnungen Art. 20 Abs. 3 VO (EG) 1107/2009.

120 S. i.E. Art. 32, 43 VO (EG) 1107/2009.

121 Eingehend dazu noch u. C I.2.c). 
178/2002, ${ }^{122}$ aufgrund derer EFSA errichtet wurde. Obwohl sich deren Begriffsbestimmungen eigentlich auf den Bereich der Lebens- und Futtermittel beziehen, spricht überwiegend viel dafür, die wortgleichen Begriffe der Pflanzenschutz-Verordnung nicht anders zu verstehen, zumal sich beide Sachbereiche überschneiden. Gemäß Art. 3 (EG) 178/2002 ist „Risiko“ eine Funktion der Wahrscheinlichkeit einer die Gesundheit beeinträchtigenden Wirkung und der Schwere dieser Wirkung als Folge der Realisierung einer Gefahr (Nr. 9) und "Gefabr" ein biologisches, chemisches oder physikalisches Agens in einem Wirkstoff oder Pflanzenschutzmittel, das eine Gesundheitsbeeinträchtigung verursachen kann (vgl. Nr. 14). „Risikobewertung“ bezeichnet einen wissenschaftlich untermauerten Vorgang mit den vier Stufen Gefahrenidentifizierung, Gefahrenbeschreibung, Expositionsabschätzung und Risikobeschreibung (Nr.11), „Risikomanagement" den von der Risikobewertung unterschiedenen Prozess der Abwägung strategischer Alternativen in Konsultation mit den Beteiligten unter Berücksichtigung der Risikobewertung und anderer berücksichtigenswerter Faktoren und gegebenenfalls der Wahl geeigneter Präventionsund Kontrollmöglichkeiten (Nr. 12).

Soweit es die Wirkstoffgenehmigung betrifft, müssen sowohl bei Erst- als auch bei Erneuerungsanträgen die Genehmigungskriterien des Art. 4 der Verordnung (EG) 1107/2009 erfüllt sein. Bei Erneuerungsanträgen genügt es, dass die Erfüllung der Anforderungen des Art. 4 VO [EG] 1107/2009 in Bezug auf mindestens einen repräsentativen Verwendungszweck bei mindestens einem Pflanzenschutzmittel nachgewiesen wird (Art. 14 Abs. 1 Satz 2 VO [EG] 1107/2009). ${ }^{123}$

Für alle Einzelregelungen der europäischen Pflanzenschutzverordnung gelten die allgemeinen Zielvorgaben des Art. 1 VO (EG) 1107/2009. Ziel der Verordnung ist „die Gewäbrleistung eines hohen Schutzniveaus für die Gesundheit von Mensch und Tier und für die Umwelt und das bessere Funktionieren des Binnenmarkts" (Art. 1 Abs. 3). Alle Vorschriften beruhen auf dem Vorsorgeprinzip (Art. 1 Abs. 4 VO). ${ }^{124}$ Art. 4 VO (EG) 1107/2009, der die zentralen Genehmigungskriterien enthält, ist dem entsprechend im Lichte dieser Vorgaben auszulegen und anzuwenden.

\section{a) Anforderungen an den Wirkstoff}

Soweit es den Wirkstoff (z.B. Glyphosat) betrifft, muss in einer bestimmten Reihenfolge geprüft werden, ob dieser die Anforderungen aus Anhang II zur VO (EG) 1107/2009 erfüllt (Art. 4 Abs. 1 Sätze 2, 3 VO [EG] 1107/2009).

Im ersten Schritt sind anhand des Anhangs II bestimmte Gefahren für die menschliche Gesundheit abzuklären: Der Wirkstoff darf weder mutagen (gentoxisch), noch karzinogen noch reproduktionstoxisch, und zwar jeweils der Kategorien 1A oder 1B

122 Verordnung (EG) Nr. 178/2002 des Europäischen Parlaments und des Rates vom 28.01.2002 zur Festlegung der allgemeinen Grundsätze und Anforderungen des Lebensmittelrechts, zur Errichtung der Europäischen Behörde für Lebensmittelsicherheit und zur Festlegung von Verfahren zur Lebensmittelsicherheit, ABl. (EG) L 31 v. 01.02.2002, S. 1.

123 Vgl. zur Erstgenehmigung Nr. 2.1 des Anhangs II zur VO (EG) 1107/2009.

124 Zum Vorsorgeprinzip noch u. E I. 
i.S.d. Verordnung (EG) 1272/2008 ${ }^{125}$ sein, es sei denn, dass ein Kontakt mit Menschen ausgeschlossen ist und Rückstände in Nahrungs- und Futtermitteln die festgelegten Standardwerte nicht überschreiten (Ausschlusskriterium). ${ }^{126}$ Hätte sich im Wiedergenehmigungsverfahren die Bewertung der Internationalen Agentur für Krebsforschung (IARC) durchgesetzt, dass Glyphosat als „wahrscheinlich krebserregend für Menschen (Gruppe 2A) " einzustufen sei, ${ }^{127}$ hätte der Antrag auf erneute Genehmigung jedenfalls nicht zwingend abgelehnt werden müssen und sogar noch ausnahmsweise ${ }^{128}$ genehmigt werden dürfen. Nicht einmal dazu ist es bekanntlich gekommen. ${ }^{129}$ Ferner müssen der Verbleib und das Verhalten des Wirkstoffs in der Umwelt bestimmte Anforderungen im Hinblick auf die kritischen Kriterien Persistenz, Bioakkumulation, Potenzial zum Ferntransport und Toxizität erfüllen, dürfen also das im Anhang definierte Maß nicht überschreiten. ${ }^{130}$

Im zweiten Schritt wird geprüft, ob der Wirkstoff bestimmte weitere Anforderungen des Anhangs II erfüllt. Dazu zählen vor allem das Fehlen endokrinschädlicher Eigenschaften, soweit sie nicht vernachlässigbar sind (Nr. 3.6.5 Anhang II), ferner umweltrelevante Anforderungen in den Problembereichen „Ökotoxikologie“ (u.a. Auswirkungen auf nicht zu bekämpfende Organismen wie z.B. Bienen), „Rückstände“ (soweit messbar) oder „Verbleib und Verhalten im Grundwasser“, aber auch Vorkehrungen zum Schutz besonders anfälliger Personengruppen oder die ordnungsgemäße Dokumentation in den Antragsdossiers. ${ }^{131}$

\section{b) Anforderungen an mindestens ein Pflanzenschutzmittel, das den Wirkstoff enthält („repräsentative Formulierung “)}

Die Wirkstoffgenehmigung beschränkt sich aber nicht nur auf den eigentlichen Wirkstoff (z.B. Glyphosat). Denn gemäß Art. 4 Abs. 1 Satz 1 VO (EG) 1107/2009 wird ein Wirkstoff gemäß Anhang II genehmigt, wenn „aufgrund des wissenschaftlichen und technischen Kenntnisstandes zu erwarten ist", dass unter Berücksichtigung der Genehmigungskriterien in Nrn. 2 und 3 dieses Anhangs „Pflanzenschutzmittel, die diesen Wirkstoff enthalten“, die Voraussetzungen der Absätze 2 und 3 des Art. 4 VO (EG) 1107/2009 erfüllen. Dies muss, wie erwähnt, bei Erneuerungsanträgen für mindestens ein repräsentatives Pflanzenschutzmittel, das den Wirkstoff enthält (z.B. „Roundup Ultra“), nachgewiesen werden (Art. 14 Abs. 1 Satz 2 VO [EG] 1107/2009). Damit findet zumindest in Bezug auf ein Pflanzenschutzmittel eine Bewertung auch auf europäischer Ebene statt, obwohl deren Zulassung den Mitgliedstaaten obliegt.

125 Fn. 71. S. i.e. Teil 3 des Anhangs zur Verordnung, Tabelle 3.6.1.

126 S. i.E. Art. 4 Abs. 1 Satz 2 i.V.m. Nrn. 3.6.2 bis 3.6.4 Anhang II zu VO (EG) 1107/2009.

127 S.o. A II. m.N.

128 Die Ausnahmeklausel des Art. 4 Abs. 7 UAbs. 2 VO (EG) 1107/2009 (Genehmigung aufgrund einer nicht anders abwendbaren Gefahr für die Pflanzengesundheit) greift im Falle der Kategorien 1A und 1B ausdrücklich nicht ein.

129 S.u. C I.3.a).

130 S. i.E. Art. 4 Abs. 1 Satz 2 i.V.m. Nr. 3.7 Anhang II zu VO (EG) 1107/2009.

131 Art. 4 Abs. 1 Satz 3 i.V.m. allen übrigen Anforderungen aus Nrn. 2 und 3 des Anhangs II zu VO (EG) 1107/2009, die nicht schon im ersten Schritt zu prüfen waren. 
Auf diese Weise soll ein gewisser Realitätsbezug der Bewertung des Wirkstoffs hergestellt werden, der ja in aller Regel nicht als reiner Stoff, sondern in einer bestimmten Formulierung ausgebracht wird.

Im Glyphosat-Wiedergenehmigungsverfahren diente „MON 52276“ als „repräsentative Formulierung“. ${ }^{132}$ Welche Rolle dieses Mittel in der Praxis tatsächlich spielt, ist nicht ermittelbar. Es gibt allerdings aus den sogenannten „Monsanto-Papers“133 Hinweise darauf, dass MON 52276 ausgewählt wurde, weil es als oberflächenaktiven Stoff (surfactant) „Dodigen“ enthielt und sich darin von anderen Formulierungen mit anderen Beistoffen (z.B. „Roundup“) unterschied, bei denen in Studien cytotoxische Wirkungen festgestellt worden waren. An solchen Vorüberlegungen zur Auswahl eines Mittels, das sich im Prüfverfahren als „free and clear“ erweisen würde, waren offenbar auch deutsche Stellen beteiligt. ${ }^{134}$

Unter den vielfältigen Anforderungen an mindestens ein repräsentatives Pflanzenschutzmittel sollen hier nur die wichtigsten erwähnt werden:

Gemäß Art. 4 Abs. 3 lit. b dürfen Pflanzenschutzmittel „keine schädlichen Auswirkungen auf die Gesundheit von Menschen", einschließlich besonders gefährdeter Personengruppen, von Tieren oder auf das Grundwasser haben. Erfasst werden dabei sofortige wie verzögerte, direkte wie indirekte Auswirkungen, etwa über das Trinkwasser, die Nahrung oder die Luft. Dabei sind auch schädliche Kumulations- und Synergieeffekte zu berücksichtigen, soweit sie durch anerkannte wissenschaftliche Methoden bewertbar sind (sogenannter „Methodenvorbehalt").

Ferner dürfen gemäß Art. 4 Abs. 3 lit. e Pflanzenschutzmittel „keine unannebmbaren Auswirkungen auf die Umwelt" haben, soweit es die folgenden Aspekte betrifft: Verbleib und Ausbreitung in der Umwelt, insbesondere Kontamination von Gewässern, Luft und Boden, Auswirkung auf Arten, die nicht bekämpft werden sollen, sowie auf die biologische Vielfalt und das Ökosystem. Insbesondere die Bedrohung der biologischen Vielfalt spielt bei Glyphosat bzw. glyphosathaltigen Mitteln eine große Rolle.

Erwähnenswert ist schließlich Art. 4 Abs. 3 lit.c, wonach Pflanzenschutzmittel „keine unannebmbaren Auswirkungen auf Pflanzen oder Pflanzenerzeugnisse" haben dürfen. Im Falle glyphosathaltiger Mittel ist vor allem an die Beeinflussung von Pflan-

132 S. etwa EFSA-Schlussfolgerung, (Fn. 1), Anhang A, S. $32 \mathrm{ff}$.

133 S.u. C I.4.c).

134 Siehe aus den „Monsanto-Papers“ die Mail von Donna R. Farmer (at MONSL125) vom 17.04.1999 betreffend "Meeting Minutes 2/25", worin sie Informationen zum aktualisierten "German Addendum" weiterleitet: "... We understand that the Germans current position on the effects observed in the various studies with the formulations [sic] as described in the open literature do not indicate a mutagenic response but rather a cytotoxic response associated with the surfactant(s). Glyphosate, it's [sic] salts, the G3 and G4 formulations (with the Dodigen surfactant) and Rodeo are free and clear. For those formulations/surfactants that can be tested up to the limit levelsper OECD guidelines and produce no toxicity such as the Dodigen (the major surfactant in MON 52276) they would be viewed favorably. Roundup (with MON 0818), Roundup Ultra, the etheramine-based formulations and other formulations either do not meet this standard or the ossiblity that they will is low.", abrufbar auf der Homepage der Anwaltskanzlei "Baum Hedlund Law" unter: www.baumhedlun dlaw.com (15.06.2019). 
zen benachbarter „glyphosatfreier“ Betriebe, aber auch an die Entstehung von Resistenzen in Unkräutern und Nutzpflanzen zu denken.

Vergleicht man die Formulierungen in Bezug auf die einzelnen Schutzobjekte, fällt die unterschiedliche Intensität der verbotenen Auswirkungen auf: Bei den Gesundheitsgefahren für Menschen sind „schädliche“ Auswirkungen unzulässig, bei den genannten Umweltgefahren dagegen nur „unannehmbare“ Auswirkungen. Es genügt also nicht, dass das Zielobjekt Umwelt Schaden nimmt. Vielmehr darf abgewogen werden, ob die schädliche Folge mit Blick auf die Vorteile des Pflanzenschutzmittels hingenommen werden kann. ${ }^{135}$

\section{c) Bewertungsgrundsätze, insbesondere Grundsätze der Transparenz und Wissenschaftlichkeit}

Die europäische Bewertungspraxis unterscheidet zwischen der Einstufung eines Stoffes und der Risikobewertung. Die „Einstufung“ eines Stoffs (oder Gemischs) betrifft die Art und Schwere der ihm inhärenten Gefahr. Dagegen setzt die „Risikobewertung“ eine bestimmte Gefahr mit der tatsächlichen Exposition von Menschen oder der Umwelt gegenüber dem Stoff, der diese Gefahr aufweist, in Beziehung. Beide sind aber auch miteinander verbunden, weil die Gefabrenermittlung und die Gefabrenbeurteilung den gemeinsamen Nenner sowohl für die Einstufung als auch für die Risikobewertung darstellen. ${ }^{136}$

Alle mit der Bewertung befassten Behörden, hier insbesondere EFSA und das deutsche BfR, sind verpflichtet, ihre Bewertung unabhängig, objektiv, transparent und unter Einschaltung von Sachverständigen nach wissenschaftlichen Grundsätzen und unter Berücksichtigung des neuesten Standes von Wissenschaft und Technik durchzuführen. Um diese Grundsätze auszuformen und sicherzustellen, hat die europäische Rechts- und Verwaltungspraxis ein Dickicht von Regelungen und Verwaltungsrichtlinien hervorgebracht.

Bei der Bewertung und Zulassung von Wirkstoffen gelten gemäß Nr. 3.5.3. der Anlage II ${ }^{137}$ zur VO (EG) 1107/2009 die einheitlichen Grundsätze i.S.v. Art. 29 Abs. 6 VO [EG] 1107/2009. Die genannte Vorschrift verweist zunächst auf die Anforderungen des nicht mehr vorhandenen Anhangs IV der Richtlinie 91/414/EWG sowie neuere Durchführungsverordnungen, die an die Stelle von anderen Anhängen der erstgenannten Richtlinie getreten sind. Dabei handelt es sich um die Durchführungsverordnung (EU) Nr. 546/2011 hinsichtlich der einheitlichen Grundsätze für die

135 S. i.E. Douhaire, ZUR 2017, S. $395 \mathrm{ff}$.

136 ECHA, Einführende Leitlinien zur CLP-Verordnung, Version 2.1., August 2015 („ECHA Guidance 2015“), S. 17 (sub 1.5.), abrufbar unter: https://echa.europa.eu (15.06.2019).

137 Entsprechend Art. 4 Abs. 4 VO (EG) 1107/2009 in Bezug auf mindestens ein repräsentatives Pflanzenschutzmittel, das in die Bewertung einzubeziehen ist. S.o. C I.2.b). 
Bewertung und Zulassung von Pflanzenschutzmitteln. ${ }^{138}$ Nach den Grundsätzen des Art. 29 Abs. 6 VO (EG) 1107/2009 werden auch die Wechselwirkungen zwischen Wirkstoffen und Beistoffen bei der Bewertung des Pflanzenschutzmittels berücksichtigt, wobei ökotoxikologische Kombinationswirkungen im Mittelpunkt stehen. ${ }^{139}$

Neben die einheitlichen Grundsätze hinsichtlich der Bewertung und Zulassung von Wirkstoffen bzw. Pflanzenschutzmitteln treten Leitlinien (Art.77 VO [EG] 1107/2009), die die Bewertungsstandards weiter ausformen. Im Glyphosat-Fall sind z.B. auch Leitlinien der Europäischen Chemikalienagentur (ECHA) bedeutsam geworden, da sie die Aufgabe hat, disparate Standards und Bewertungen innerhalb des europäischen Rechts nach bestimmten Regeln zu harmonisieren. ${ }^{140}$

Das Erfordernis der Wissenschaftlichkeit ist in praktisch allen genannten Regelungen und Leitlinien verankert. So wird z.B. im Anhang II zur Pflanzenschutzverordnung 1107/2009 verlangt, dass die Bewertung von Wirkstoffen durch EFSA und den berichterstattenden Mitgliedstaat „wissenschaftlichen Grundsätzen folgen“ und unter Einbeziehung von Sachverständigen vorgenommen werden muss (Nr. 1.2). Da die EU diese Grundsätze aber nicht selbst ausformt, muss insoweit auf Standards aus der Wissenschaft zurückgegriffen werden, z.B. den European Code of Conduct for Research Integrity. ${ }^{141} \mathrm{Zu}$ den wichtigsten Regeln gehört u.a., dass eine Studie keine relevanten Informationen unterschlagen darf („Falsifikation“) oder dass alle, die einen substantiellen Beitrag zur Publikation geleistet haben, auch als Autor oder Autorin erkennbar werden und die Übernahme fremder Ideen ohne Nennung der Originalquelle unterbleibt (Plagiatsverbot). Außerdem gelten in der EU die OECD-Grundsätze zur guten Laborpraxis (GLP-Grundsätze) in Bezug auf Prüflabore, die gesund-

138 Verordnung (EU) Nr. 546/2011 der Kommission vom 10. Juni 2011 zur Durchführung der Verordnung (EG) Nr. 1107/2009 des Europäischen Parlaments und des Rates hinsichtlich einheitlicher Grundsätze für die Bewertung und Zulassung von Pflanzenschutzmitteln, ABl. (EU) L 155 v. 11.06.2011, S. 127.

139 S.o. A II.

140 Bei besonders kritischen Gefahren (Kanzerogenität, Mutagenität, Reproduktionstoxizität [CMR] sowie Inhalationsallergenen) gilt das Verfahren für die harmonisierte Einstufung und Kennzeichnung (CLH). Siehe dazu Anhang VI der CLP-Verordnung(EG) 1272/2008, (Fn. 71), i.V.m. ECHA Guidance 2015“, (Fn. 136). Speziell zur Erstellung des Entwurfs des Bewertungsberichts durch den berichterstattenden Mitgliedstaat: Art. 11 Abs. 2 Satz 2 VO (EG) 1107/2009. Für EFSA und berichterstattenden Mitgliedstaat: Nrn. 1.2. und 1.3. des Anhangs II zu VO (EG) 1107/2009 i.V.m. Leitlinien des Ständigen Ausschusses für die Lebensmittelkette und Tiergesundheit. Eine Zusammenstellung aller Leitlinien aus dem Tätigkeitsbereich von ECHA findet sich auf deren Homepage (s. zuvor); s.a. die Rules of Procedure for the Scientific Committees on Consumer Safety (SCCS) and Health, Environmental and Emerging Risks (SCHEER) vom 28.04.2016, abrufbar bei der Kommission unter: https://ec.europa.eu (15.06.2019). Hier finden sich u.a. Detailregelungen über das Erfordernis und die Form der „Declaration of Interests“; speziell zur Vermeidung divergierender Ergebnisse: Common Guidelines on Practical Arrangements for the Sharing of Scientific Data Between the Scientific Committees and Panels of European Agencies and the Scientific Committees of the Commission vom 10.11.2008, abrufbar bei EFSA (s. zuvor).

141 ALLEA (All European Academies), The European Code of Conduct for Research Integrity, revised edition, Berlin, 2017. Darauf stützt sich z.B. die Kommission auf ihrer Forschungsseite, abrufbar unter: https://ec.europa.eu/research (15.06.2019). 
heits- und umweltrelevante Risikobewertungen vornehmen. ${ }^{142}$ Diese Standards beziehen sich vor allem auf die Sicherung der Qualität von Prüfdaten durch angemessen organisierte Prüfeinrichtungen und Prüfverfahren, Behandlung der Prüfobjekte, Dokumentation etc.

Die Objektivität und Unabbängigkeit der Bewertung wird in den Leitlinien durch Vorkehrungen wie z.B. „Declarations of Interest“ (DoI) abgesichert, in der die eingeschalteten Experten und Expertinnen Angaben zu möglichen Interessenkonflikten machen und mögliche Verbindungen mit den antragstellenden Unternehmen offenlegen müssen. Solche Formularmechanismen mögen dazu beitragen, offenkundig mit antragstellenden Unternehmen verbandelten Fachleuten den Zugang zur Risikobewertung zu versagen. Ansonsten dürften sie aber wohl ähnlich einzuschätzen sein wie die Selbstbekundungen von Doktoranden, dass sie bei der Anfertigung ihrer Dissertation alle Regeln eingehalten hätten.

Besonders wichtig ist der Grundsatz der Transparenz, weil das ordnungsgemäße Zustandekommen wie auch die sachliche Richtigkeit der Bewertung ohne die Offenlegung der relevanten Informationen nicht von außen (Wissenschaft, Medien und Öffentlichkeit) kontrolliert werden können. Insoweit ist die Rechtslage allerdings stark vom Schutz des Geschäfts- und Betriebsgeheimnisses und potentieller Offenlegungsopfer geprägt. Gemäß Art. 8 der Pflanzenschutzmittel-Verordnung (EG) 1107/2009 müssen die Antragsteller ihren Antrag auf (Wieder-)Genehmigung mit eigenen Studien („Originalstudien“) zum Wirkstoff und mindestens einem Pflanzenschutzmittel untermauern, auf die dann auch die Bewertungsberichte zurückgreifen. Die Studien und weitere Informationen, die von der Industrie für die Bewertung von Wirkstoffen und Pflanzenschutzmitteln vorgelegt werden müssen, sind sogenannte „Datenanforderungen“, für die spezielle Vorschriften gelten. ${ }^{143} \mathrm{Obwohl}$ die Unternehmensstudien ausschlaggebend für die Transparenz sind, können Antragsteller beantragen, sie als vertrauliche Informationen zu behandeln (Art. 7 Abs. 3, Art. 12 Abs. 1 Satz 3 VO [EG] 1107/2009). Sie müssen dann nachweisen, dass die Offenlegung dieser Informationen ihre wirtschaftlichen Interessen oder den Schutz ihrer Privatsphäre oder ihre Integrität beeinträchtigen könnte, was in einigen Fällen sogar vermutet wird (Art. 63 VO [EG] 1107/2009). Es entscheidet der zuständige Mitgliedstaat, bei Anträgen auf Informationszugang der berichterstattende Mitgliedstaat (Art. 7 Abs. 3 VO [EG] 1107/2009).

Vertrauliche Informationen bleiben auch im Erneuerungsverfahren vertraulich, es sei denn, es besteht ein übergeordnetes öffentliches Interesse an der Offenlegung

142 Richtlinie 2004/10/EG des Europäischen Parlaments und des Rates vom 11. Februar 2004 zur Angleichung der Rechts- und Verwaltungsvorschriften für die Anwendung der Grundsätze der Guten Laborpraxis und zur Kontrolle ihrer Anwendung bei Versuchen mit chemischen Stoffen, ABl. (EG) L 50 v. 20.02.2004, S. 44, i.V. mit den GLP-Grundsätzen der OECD in Anhang I; Richtlinie 2004/9/EG des Europäischen Parlaments und des Rates vom 11. Februar 2004 über die Inspektion und Überprüfung der Guten Laborpraxis (GLP), ABl. (EG) L 50 v. 20.02.2004, S. 28. Eine Zusammenstellung der OECD-Regeln bietet das BfR unter: www.bfr.bund.de/de/oecd_dokumente_zur_guten_laborpraxis-473 .html (15.06.2019).

143 S.o. Fn. 109. 
(Art. 16 VO [EG] 1107/2009). Da die Vertraulichkeit „unbeschadet“ der Richtlinie 2003/4/EG über den Zugang der Öffentlichkeit zu Umweltinformationen gilt, können Streitigkeiten über die Reichweite der Geheimhaltung zwar in einem förmlichen Verfahren überprüft werden. ${ }^{144}$ Das ändert aber nichts daran, dass der Zugang zu den industrieunterstützten Studien bislang in großen Teilen unzugänglich ist und die behördennotorische Tendenz zur Ausweitung der Vertraulichkeit ihr Übriges tut. So kommt es z.B. vor, dass selbst der Name einer möglicherweise involvierten Consulting-Firma als vertrauliche Information klassifiziert wird. ${ }^{145}$ Auf der Internetseite des BVL sind die Dokumente zur Wirkstoffbewertung betreffend Glyphosat nur auf einer CD-ROM erhältlich, weil die Dateigröße insgesamt $25 \mathrm{MB}$ überschreite. ${ }^{146}$ Wenn man diese CD-ROM anfragt, wird man auf einen Link auf der Homepage von EFSA verwiesen, die dann ein mit persönlichen Daten auszufüllendes Formular präsentiert. ${ }^{147}$ Die Studien, die EFSA zur Glyphosatbewertung verfügbar macht, können nur mithilfe einer Gebrauchsanweisung gefunden werden. ${ }^{148}$ Transparenz sieht anders aus.

Das Problem des Umgangs mit Unternehmensstudien, deren Offenlegung Geschäfts- und Betriebsgeheimnisse verhindern, hat im Glyphosat-Wiedergenehmigungsverfahren eine bedeutende Rolle gespielt. Im März 2016 hatten Mitglieder des Europäischen Parlaments öffentlichen Zugang zu den kompletten Aufzeichnungen über Tierlabordaten im Zusammenhang mit verwerteten Studien über die Gentoxizität und Karzinogenität von Glyphosat verlangt. Die betreffenden Daten waren zum Teil als vertrauliche Geschäftsgeheimnisse eingestuft worden, zum Teil waren die Studien in einer wissenschaftlichen Studie von $2015^{149}$ - mehr oder weniger - dargestellt. Die förmliche Anfrage führte zwar dazu, dass die Kommission die Rohdatenverzeichnisse freigab. ${ }^{150}$ Bedeutende Teile der Sicherheitsakte, nämlich Materialien und Methoden, Analyse- und Diskussionsteile, blieben aber weiterhin verschlossen. Deshalb lautete die Kritik externer Wissenschaftler, dass man jetzt zwar Rohdaten

144 S.u. E II.1.

145 S.u. C I.4.b).

146 Abrufbar unter: www.bvl.bund.de/DE/04_Pflanzenschutzmittel/01_Aufgaben/03_EU Wirkstoffpruefung/02_info_wirkstoffe/info_wirkstoffe_dokumente/psm_euWirkstoffpr uefung_psm_info_wirk_dok_node.html (15.06.2019).

147 In seiner Antwort vom 08.01.2019 an die Autorin dieses Beitrags bietet das BVL eine CDROM in Bezug auf den „Draft Assessment Report (vorher: Monograph) von 1998“ an und verweist im Übrigen auf den Link http://dar.efsa.europa.eu/dar-web/provision (15.06.2019). Dort müssen aber erst Angaben zu Person, Institution und Adresse etc. gemacht werden.

148 Man muss auf der EFSA-Homepage unter "Register of Questions” (http://registerofque stions.efsa.europa.eu [15.06.2019]) den Reiter „Output“ ansteuern und dort unter „Title“ den Suchbegriff "Glyphosate“ eingeben.

149 Greim et al., Critical Reviews in Toxicology 2015/45, S. 185-208. Auf diese Veröffentlichung beriefen sich EFSA und ECHA auch in der „Portier-Kontroverse” (s.u. C I.4.a).

150 Siehe Pressemeldung der EFSA vom 16.12.2009 („Glyphosat: EFSA gibt Rohdaten aus Risikobewertung frei“), abrufbar unter: www.efsa.europa.eu/de/press/news/161209 (15.06.2019). 
habe, es aber nach wie vor nicht möglich sei, die Qualität und Ergebnisse von der Risikobewertung zugrunde liegenden Unternehmensstudien zu überprüfen. ${ }^{151}$

Hinzu kamen Vorwürfe der Studienmanipulation im Zusammenhang mit den sogenannten „Monsanto Papers“. ${ }^{152}$ Das Europäische Parlament verwies in diesem Zusammenhang auf die große Bedeutung der Transparenz und öffentlichen Verfügbarkeit wissenschaftlicher Studien einschließlich der Rohdaten. Es müsse durch entsprechende Verfahrensgestaltungen - möglicherweise durch Anwendung des „REFIT-Verfahrens“ gemäß Verordnung (EG) 1107/2009 - sowie hinreichende Ausstattung der befassten Behörden sichergestellt werden, dass sich die wissenschaftliche Bewertung von Pestiziden für die Genehmigung „ausschließlich auf veröffentlichte überprüfte und unabhängige Studien stützt, die von den zuständigen Behörden in Auftrag gegeben“" worden sind. ${ }^{153}$

Gewisse Veränderungsprozesse sind immerhin im Gange. Denn eine wesentliche Reaktion der Europäischen Kommission auf die erfolgreiche Einreichung der Bürgerinitiative „Verbot von Glyphosat”154 bestand in der Ankündigung, die Transparenz der Studien, die der Wirkstoffgenehmigung zugrunde liegen, zu verbessern. ${ }^{155}$ Dabei hat sie allerdings auch klargemacht, dass „weiterhin ein ausgewogenes Verhältnis zwischen den rechtmäßigen Interessen der Betroffenen, die größtmögliche Transparenz fordern, und dem Schutz legitimer Geschäftsgeheimnisse der Antragsteller in den Genehmigungsverfahren der EFSA sichergestellt" werden müsse. ${ }^{156}$

Inzwischen liegt nicht nur der versprochene Entwurf eines Legislativakts vor, ${ }^{157}$ sondern wurde auch eine Einigung zwischen Parlament und Ratsvorsitz über eine neue Verordnung erreicht, die noch der formalen Zustimmung beider Institutionen bedarf (Stand: 26.2.2019). Danach muss EFSA alle Studien veröffentlichen, die von der Industrie im Genehmigungsverfahren von Pestiziden, gentechnisch veränderten Produkten und Lebensmittelzusatzstoffen eingereicht werden. Es wird eine gemeinsame europäische Datenbank für Auftragsstudien eingerichtet, um Unternehmen daran zu hindern, für sie ungünstige Studien zurückzuhalten. Außerdem kann die EU-

151 Siehe Offener Brief von Christopher Portier vom 28.05.2017, S. 4 f. Dazu noch u. C I.4.a).

152 S.u. C I.4.c).

153 Auch zum Folgenden Erwägungsgrund J sowie Ziffer 7 der Entschließung des Europäischen Parlaments vom 24.10.2017, (Fn. 92). S.o. B I.3. m.N.

154 S.o. B I.2.b).

155 Mitteilung der Kommission vom 12.12.2017, C (2017) 8414 final, insbes. S. 16.

156 Ibid., S. 12.

157 Der Verordnungsentwurf vom 11.04.2018 richtet sich auf die Änderung zahlreicher Sekundärrechtsakte: Proposal for a Regulation of the European Parliament and of the Council on the transparency and sustainability of the EU risk assessment in the food chain amending Regulation (EC) No. 178/2002 [on general food law], Directive 2001/18/EC [on the deliberate release into the environment of GMOs], Regulation (EC) No. 1829/2003 [on GM food and feed], Regulation (EC) No. 1831/2003 [on feed additives], Regulation (EC) No. 2065/2003 [on smoke flavourings], Regulation (EC) No. 1935/2004 [on food contact materials], Regulation (EC) No. 1331/2008 [on the common authorisation procedure for food additives, food enzymes and food flavourings], Regulation (EC) No. 1107/2009 [on plant protection products] and Regulation (EU) No. 2015/2283 [on novel foods], $\operatorname{COM}(2018)$ 179, 2018/0088 (COD), abrufbar unter: https://ec.europa.eu/food/sites/ food/files/gfl_transparency_comm_proposal_20180410_en.pdf (15.06.2019). 
Kommission die EFSA in Ausnahmefällen von großer gesellschaftlicher Bedeutung ersuchen, eigene Studien in Auftrag zu geben. Die Kommission versteht den Verordnungsentwurf als einen Versuch, das Vertrauen der Öffentlichkeit in das europäische Regulierungssystem sicherzustellen. ${ }^{158} \mathrm{Ob}$ das genügt und ob das die Behörden bindende Transparenzgebot mit Blick auf das Individualrecht auf Zugang zu Umweltinformationen nicht noch mehr erfordert, wird an anderer Stelle zu vertiefen sein. ${ }^{159}$

\section{Die Entscheidung zur Erneuerung der Genehmigung im Fall des Wirkstoffs Glyphosat}

\section{a) Risikobewertung}

Im Verfahren der Erneuerung der Genehmigung für den Wirkstoff Glyphosat auf der europäischen Ebene war Deutschland der berichterstattende Mitgliedstaat und die Slowakei der „berichtmiterstattende Mitgliedstaat“. ${ }^{160}$ Dem entsprechend wurde die grundlegende Bewertung, auf der das europäische Peer-Review aufbaute, durch das Bundesamt für Risikobewertung (BfR) vorgenommen. D.h. das BfR fertigte den sogenannten „Renewal Assessment Report (RAR)“ an, der in einer vorläufigen Version zunächst die öffentliche Konsultation und ein Experten-Peer Review durchlief, um dann der EFSA als maßgebliche Grundlage für deren „Schlussfolgerung" zu dienen. Deutschland übernahm damit die Verantwortung für die wissenschaftliche Bewertung der aktiven Substanz Glyphosat.

Das Verfahren der Erneuerung der Genehmigung für Glyphosat verlief nicht problemlos. ${ }^{161}$ Denn nachdem Glyphosat schon als unbedenklich im Sinne der geltenden Standards eingestuft worden war - der vorläufige Bewertungsbericht (Assessment Report) des deutschen BfR war bereits Ende 2013 an die Europäische Behörde für Lebensmittelsicherheit (EFSA) gelangt, wurde im März 2015 die öffentlichen Stellungnahme der Internationalen Agentur für Krebsforschung (IARC) publik, wonach Glyphosat "wahrscheinlich krebserregend für Menschen (Gruppe 2A)" sei. ${ }^{162}$ Weil dies zu ungewöhnlich vielen Reaktionen aus den Mitgliedstaaten und der Öffentlichkeit bis hin zur erfolgreichen Bürgerinitiative gegen Glyphosat führte und auch das Europäische Parlament den Ausstieg aus der Glyphosatverwendung forderte, ${ }^{163}$ beauftragte die Europäische Kommission die EFSA, sich mit den Ergebnissen der Internationalen Agentur für Krebsforschung auseinanderzusetzen und das Ergebnis in

158 Mitteilung der Kommission vom 12.12.2017, (Fn. 155), S. 12.

159 S.u. E II.1.

160 Siehe Anhang I der Verordnung (EU) Nr. 1141/2010 der Kommission vom 7. Dezember 2010 zur Festlegung des Verfahrens für die erneute Aufnahme einer zweiten Gruppe von Wirkstoffen in Anhang I der Richtlinie 91/414/EWG des Rates und zur Erstellung der Liste dieser Wirkstoffe, ABl. (EU) L 322 v. 08.12.2010, S. 10.

161 Die folgenden Informationen zum Verfahrensverlauf sind den Erwägungsgründen zur Durchführungsverordnung (EU) 2017/2324 der Kommission vom 12. Dezember 2017 zur Erneuerung der Zulassung des Wirkstoffs Glyphosat, (Fn. 74), entnommen.

162 S.o. A II.

163 S.o. B I.2.b). 
die eigene Schlussfolgerung aufzunehmen. Auch das BfR musste seinen endgültigen Bewertungsbericht noch einmal im Lichte der IARC-Ergebnisse überarbeiten. Fristen für das Verfahren wurden dem entsprechend verlängert. ${ }^{164}$

Im Oktober 2015 legte die EFSA den fast 2000 Seiten umfassenden Reer Review Report on Glyphosate ${ }^{165}$ vor, der zahlreiche, zum Teil geschwärzte Ergebnistafeln, Meeting Reports, vor allem aber die Stellungnahmen von „Stakeholdern“ (Mitgliedstaaten, EU-Institutionen, Unternehmen, NGOs) umfasst. Diese beziehen sich sowohl auf den Bewertungsbericht (Renewal Assessment Report [RAR]) des BfR als auch auf den aktualisierten Entwurf der EFSA-Schlussfolgerung (EFSA Conclusion).

Noch im Oktober 2015 übermittelte EFSA ihre Schlussfolgerung (Conclusion) zum Peer Review an die Europäische Kommission, wonach Glyphosat die Anforderungen des Art. 4 VO (EG) 1107/2009 erfülle. ${ }^{166}$ Es sei insbesondere „unwabrscheinlich“, dass Glyphosat karzinogen wirke. ${ }^{167}$ Weil es weder als karzinogen noch reproduktionstoxisch der Kategorie 2 im Sinne dieser Verordnung bewertet wurde, waren zu diesem Zeitpunkt zugleich auch die genehmigungshemmenden Kriterien der Interimsbestimmungen aus Nr. 3.6.5 des Anhangs II zur VO (EG) 1107/2009 nicht erfüllt, wonach ein Wirkstoff keine endokrinschädlichen Eigenschaften haben darf. ${ }^{168}$ Im September 2017 übermittelte EFSA dann im Nachgang die weitere Schlussfolgerung, wonach auch nach den inzwischen vorhandenen spezifischen Bewertungskriterien keine potentiell endokrinen (hormonellen) Wirkungen in Bezug auf Glyphosat festgestellt werden könnten. ${ }^{169}$ Zwar konnten gewisse Effekte beim Schlüpfen von Larven beobachtet werden. Diese wurden aber für irrelevant erklärt, weil sie nur für Glyphosat und nicht für „Roundup“ statistisch signifikant seien. ${ }^{170}$ Soweit es Auswirkungen von Glyphosat auf die Umwelt betrifft, erscheinen vor allem die Angaben aus der EFSA-Schlussfolgerung von $2015 \mathrm{zu}$ den Funden von Glyphosat/AMPA in Wasser und Boden interessant. ${ }^{171}$ Insbesondere in Italien, Deutschland, den Niederlanden, Schweden, Frankreich und Spanien wurden messbare Glyphosatmengen (> 0,1 Mikrogramm/Liter) im Grundwasser gefunden, wenn auch in sehr geringen Mengen und bei einem Teil der Proben. In Frankreich, den Niederlanden, im Vereinigten Königreich und Schweden wurden darüber hinaus auch einzelne Überschreitungen

164 Siehe Ranzenmayr, S. $42 \mathrm{f}$.

165 EFSA, Reer Review Report on Glyphosate vom 20.10.2015, abrufbar unter: http://regist erofquestions.efsa.europa.eu/roqFrontend/wicket/page?2 (15.06.2019).

166 EFSA-Schlussfolgerung, (Fn. 1).

167 Ibid., S. 4302: "... EFSA concluded that glyphosate is unlikely to pose a carcinogenic hazard to bumans and the evidence does not support classification with regard to its carcinogenic potential according to Regulation (EC) No 1272/2008".

168 Ibid., S. 12. Die Übergangsbestimmungen in Nr.3.6.5 des Anhanges II sehen vor, dass kanzerogene und reproduktionstoxische Stoffe der Kategorie 2 als „endokrinschädlich“ gelten, bis die Kommission „Maßnabmen in Bezug auf konkrete wissenschaftliche Kriterien zur Bestimmung der endokrinschädlichen Eigenschaften" ergriffen hat. Spezifische Kriterien lagen bis zur EFSA Conclusion noch nicht vor, weshalb sie erst im Nachgang berücksichtigt wurden.

169 EFSA, EFSA Journal 2017/15(9):4979; DOI: 10.2903/j.efsa.2017.4979, insbes. S. 7.

170 Ibid., S. 8.

171 Auch zum Folgenden EFSA-Schlussfolgerung, (Fn. 1), S. 18 und Anhang A (S. 83 ff.). 
der zulässigen Höchstmenge im Trinkwasser festgestellt. Allerdings sind dies auch die Länder, für die detaillierte Grundwasserbeobachtungsstudien überhaupt die nötigen Daten lieferten. Bezüglich des Bodenmonitoring findet sich in der EFSA-Schlussfolgerung nur der Eintrag "no data“. Allein aus den noch lückenhaften Bestandsaufnahmen zum Grundwasser lässt sich aber schon ableiten, dass Glyphosat - wenn auch in noch geringen Mengen - die Natur einschließlich des Grundwassers erreicht hat und zunehmend durchdringt. Dabei scheint sich abzuzeichnen, dass in Gebieten mit intensiver landwirtschaftlicher Nutzung höhere Belastungen des Wassers einhergehen. Völlige Klarheit besteht angesichts der lückenhaften Datenlage aber nicht.

Erst nach der EFSA Schlussfolgerung lag im Oktober 2015 die finale Fassung des vom deutschen BfR erstellten, mehrfach überarbeiteten „Renewal Assessment Report (RAR)“172 vor, mit etlichen geschwärzten Angaben auf über 4.300 Seiten. Das BfR setzte sich im RAR auch mit dem Ergebnis der IARC auseinander, wonach Glyphosat „wahrscheinlich kanzerogen für Menschen "173 sei. Seiner Ansicht nach ist die Aussagekraft der epidemiologischen Studien, die das IARC herangezogen hatte, limitiert, weil sie „keinen übereinstimmenden Beweis für einen klaren und starken Zusammenhang" lieferten. ${ }^{174}$ Zwar räumt das BfR ein, dass z.B. eine Studie von Schinasi und Leon (2014) einen möglichen Zusammenhang zwischen einer Unterart des NonHodgkin-Lymphoms (B-Zell-Lymphom) und Glyphosat aufzeige. ${ }^{175}$ Die Studien ließen aber nicht hinreichend klar erkennen, welche Anwendungsprodukte ihnen zugrunde lagen. Selbst dort, wo „Roundup“ genannt sei, sei nicht ausdrücklich klargestellt, ob nicht vielleicht auch andere Formulierungen verwendet wurden. Deshalb könne ein möglicher Einfluss von Co-Beistoffen in den einzelnen Formulierungen auf das Ergebnis der Studien nicht ausgeschlossen werden. Statt also auf Präzisierung zu dringen, um sich anhand eines geklärten Versuchsaufbaus mit den Ergebnissen der Studie auseinanderzusetzen, leitet das BfR aus fehlender Präzisierung die Unbedenklichkeit ab. Die „OR“ [odds ratio ${ }^{176}$ ] zwischen der Glyphosat-Exposition und dem Auftreten des Non-Hodgkin-Lymphoms sei zwar „leicht erhöht“, aber „,in den meisten Fällen nicht signifikant“. Es gäbe „sicherlich keinen »klaren Beweis «”. ${ }^{177}$ Zwar

172 Final addendum to the Renewal Assessment Report (public version) of 19 November 2015, Risk assessment provided by the rapporteur Member State Germany and co-rapporteur Member State Slovakia for the active substance GLYPHOSATE according to the procedure for the renewal of the inclusion of a second group of active substances in Annex I to Council Directive 91/414/EEC laid down in Commission Regulation (EU) No. 1141/2010, abrufbar bei EFSA unter: http://registerofquestions.efsa.europa.eu/roqFrontend/output Loader?output=ON-4302 (15.06.2019).

173 S.o. A II. m.N.

$174 R A R$,(Fn. 172), S. 4139: "no une quivocal evidence for a clear and strong association of NHL with glyphosate".

175 RAR, (Fn. 172), S. 4136: "Schinasi and Leon (2014, [17]) published the results of epidemiologic research on the relationship between NHL and occupational exposure to pesticides... Here, in a metaanalysis based on the studies by Eriksson (2008, [13]) and Cocco et al. (2012, [18]), an association between a subtype of NHL (B-cell lymphoma) and glyphosate could be elicited".

176 „Odds ratio“ (OR) ist ein Assoziationsmaß, das die Stärke eines Zusammenhangs von zwei Merkmalen angibt.

177 RAR, (Fn. 172), S. 4137. 
hielt das BfR weitere Forschung hinsichtlich der tatsächlich verwendeten GlyphosatFormulierungen für notwendig. ${ }^{178}$ Im Ergebnis gelangte es aber zu folgender Bewertung:

„Das BfR kommt nach Prüfung aller bislang vorliegenden Studien, Dokumente und Veröffentlichungen einschließlich der Glyphosat-Monographie der Internationalen Agentur für Krebsforschung der WHO (IARC) zu dem Ergebnis, dass nach derzeitiger wissenschaftlicher Kenntnis bei bestimmungsgemäßer Anwendung von Glyphosat kein krebserzeugendes Risiko für den Menschen zu erwarten ist. Diese Auffassung vertreten mit einer Ausnahme (Schweden) auch die Experten aus den EU-Mitgliedstaaten und die EFSA in der nunmehr veröffentlichten Schlussfolgerung (EFSA - Conclusion) im laufenden Verfahren der EU-Wirkstoffprüfung zur weiteren Genehmigung von Glyphosat als Wirkstoff für Pflanzenschutzmittel.“179

Danach wird das Krebsrisiko mit zwei gewichtigen Einschränkungen ausgeschlossen: erstens handele es sich um eine Einschätzung „nach derzeitiger wissenschaftlicher Kenntnis“ und zweitens gälte sie nur „bei bestimmungsgemäßer Anwendung von Glyphosat“. Wie schon das Zitat angibt, hat sich nur Schweden klar gegen diese Risikobewertung des BfR positioniert. Soweit es andere Risiken als das Krebsrisiko betrifft, werden im RAR aber durchaus „critical areas of concern“ identifiziert. Dazu zählt insbesondere das Risiko für die Biodiversität und die mögliche Toxizität von Beistoffen, was aber nur zur Folge hat, dass die Mitgliedstaaten zum Ergreifen von Maßnahmen bzw. weiterer Aufklärung aufgefordert werden. ${ }^{180}$ Im weiteren Verlauf veröffentlichten Mitarbeiter von EFSA und BfR einen Beitrag in einer Fachzeitschrift, in der sie die Unterschiede zwischen den Ergebnissen des europäischen Peer Review und denjenigen der IARC mit der Nutzung unterschiedlicher Datensets und unterschiedlicher Bewertungsmethoden erklärten. ${ }^{181}$

Im Januar 2016 legte die Kommission dem Ständigen Ausschuss für Pflanzen, Tiere, Lebens- und Futtermittel den ersten Entwurf ihres Überprüfungsberichts (Art. 13 Abs. 1 VO [EG] 1107/2009) für Glyphosat vor. ${ }^{182}$ Wegen der disparaten Einschätzungen zog dieser es allerdings vor, eine Stellungnahme des Ausschusses für Risikobeurteilung (Risk Assessment Committee [RAC]) der Europäischen Chemikalien-

$178 R A R$, (Fn. 172), S. 4139: "Probably, further research needs to be carried out to study the usage and the impact of the formulation used in the field situation".

$179 B f R$, Fragen und Antworten zur Bewertung des gesundheitlichen Risikos von Glyphosat, A-Z-Index, FAQ des BfR vom 1. März 2016, abrufbar unter: https://mobil.bfr.bund.de (15.06.2019). Der Entscheidungsvorschlag des BfR im RAR, (Fn. 172), S. 196 f., ist komplett geschwärzt.

180 RAR, (Fn. 172), S. $190 \mathrm{f}$.

181 Tarazona et al., Archives of Toxicology 2017/91, S. 2723-2743. Zur Gegenposition Portier et al., Journal of Epidemiology and Community Health 2016/70, S. 741-745.

182 Siehe Erwägungsgrund 10 der DurchführungsVO (EU) 2017/2324 der Kommission. Zum finalen Überprüfungsbericht noch im Folgenden sowie unten C I.3.b) aa) m.N. 
agentur (ECHA $)^{183}$ „zur harmonisierten Einstufung im Hinblick auf die Karzinogenität von Glyphosat“ - den sogenannten CLH (Classification and Labelling Harmonisation) Report - einzuholen. ${ }^{184}$ Der ECHA-Ausschuss (RAC) bestätigte in seiner Stellungnahme (CLP Report) vom Mai 2016, dass für Glyphosat auf der Grundlage einer Gewichtung der Beweiskraft der Daten ("weight of evidence approach") eine „Risikoeinstufung von Glyphosat in Bezug auf Karzinogenität gemäß den CLPKriterien ${ }^{185}$ nicht geboten “ sei; andere Risiken - wie Organtoxizität und Schädlichkeit für Wasserorganismen - sah es aber durchaus. ${ }^{186}$

In der Zwischenzeit (Mai 2016) hatte auch das Joint FAO/WHO Meeting on Pesticides Residues (JMPR) eine Neubewertung von Glyphosat vorgenommen, mithilfe derer beide Organisationen einen gemeinsamen Standpunkt finden sollten. Das JMPR gelangte dabei - anders als zuvor die Internationale Agentur für Krebsforschung (IARC) - zum Ergebnis, dass eine Krebsgefahr für Menschen unwahrscheinlich sei, obwohl es durchaus Korrelationen zwischen dem Non-Hodgkin Lymphom und einer Glyphosat-Exposition zur Kenntnis nahm:

"Overall, there is some evidence of a positive association between glyphosate exposure and risk of NHL [Non-Hodgkin Lymphoma] from the case-control studies and the overall meta-analysis. However, it is notable that the AHS, ${ }^{187}$ which is the only cohort study and is large and of high quality, found no evidence of association at any exposure level.

In view of the absence of carcinogenic potential in rodents at human-relevant doses and the absence of genotoxicity by the oral route in mammals, and considering the epidemiological evidence from occupational exposures, the Meeting concluded that

183 Gemäß CLP-Verordnung (EG) 1272/2008, (Fn. 71), ist der Ausschuss für Risikobeurteilung (Risk Assessment Committee [RAC]) der ECHA für die wissenschaftliche Bewertung von Vorschlägen für eine harmonisierte Einstufung von Stoffen zuständig.

184 CLH Report, Version 2.0 (Mai 2016), abrufbar unter: https://echa.europa.eu/documents/ 10162/9fb5d873-2034-42d9-9e53-e09e479e2612 (15.06.2019).

185 S. zur CLP-Verordnung, (Fn. 71).

186 CLH Report, (Fn. 184), S. 98 (Kanzerogenität): "Based on the epidemiological data as well as on data from long-term studies in rats and mice, taking a weight of evidence approach, no hazard classification for carcinogenicity is warranted for glyphosate according to the CLP criteria"; zu den übrigen Risiken: S. 42 (Organ-Toxizität): "It is proposed to classify glyphosate as STOT RE, Category 2. ... (May cause damage to organs through prolonged or repeated exposure)"; S. 59 (Mutagene Wirkung auf Keimzellen): "No hazard classification of glyphosate for mutagenicity is warranted according to the CLP criteria"; S. 114 (Reproduktionsrisiken): "No classification and labelling of glyphosate for reproductive or developmental effects is proposed"; S. 134 (Umweltrisiken): "Glyphosat fulfils the criteria for classification as Aquatic Chronic 2".

187 AHS = Agricultural Health Study: Lerro et al., Occupational and Environmental Medicine 2015/72, S. 736-744. 
glyphosate is unlikely to pose a carcinogenic risk to humans via exposure from the diet." 188

Demzufolge ist die in Meta-Analysen festgestellte Korrelation zwischen Krebs und Glyphosatexposition nicht aussagekräftig, weil eine qualitätvolle Kohortenstudie (AHS-Studie) keinerlei Zusammenhang feststellen konnte. Wie sich die statistischen Korrelationen dann erklären könnten, lässt das JMPR offen.

Auf der Grundlage der gesammelten Erkenntnisse gelangte die Kommission zu dem Schluss, dass allein das IARC Glyphosat für wahrscheinlich krebserregend halte. Dies erkläre sich damit, dass das IARC sowohl den Wirkstoff Glyphosat als auch glyphosathaltige Pflanzenschutzmittel untersucht habe, während sich die Bewertung durch die EU auf den Wirkstoff beschränke, weil für die Bewertung der einzelnen Pflanzenschutzmittel die Mitgliedstaaten zuständig seien. Zudem betrachte das IARC nur veröffentliche Studien, während in die EU-Bewertung auch Studien einbezogen würden, „die die Antragsteller im Rahmen ihrer Anträge übermittelt haben und die nicht öffentlich zugänglich sind“, so dass „im Rahmen der EU-Bewertung mehr einschlägige Unterlagen berücksichtigt, einschließlich zentraler Studien, die vom IARC nicht einbezogen wurden“. Das erkläre die unterschiedliche Gewichtung der vorhandenen Daten durch IARC einerseits und EFSA andererseits zum größten Teil. ${ }^{189}$

Auf dieser Basis hielt die Kommission die Voraussetzungen für eine Erneuerung der Glyphosatzulassung (Art. 4, 14 Abs. 1 Satz 2 VO [EG] 1107/2009) für erfüllt und legte dem Ständigen Ausschuss für Pflanzen, Tiere, Lebens- und Futtermittel den Entwurf einer Durchführungsverordnung zur Erneuerung der Genehmigung vor. Nachdem die erforderliche Mehrheit für eine positive Stellungnahme dazu im Ständigen Ausschuss für Pflanzen, Tiere, Lebens- und Futtermittel (SCoPAFF) innerhalb der vom Vorsitz (der Kommission) gesetzten Frist nicht zustande gekommen war, entschied der Vorsitz, den Berufungsausschuss (ACoPAFF) einzuschalten, weil ein Durchführungsrechtsakt „als notwendig erachtet“ wurde. ${ }^{190}$ Mit Blick auf die schwierige Mehrheitsfindung war allerdings auch klar geworden, dass die Bedingungen für eine erneute Genehmigung nicht mehr dieselben bleiben konnten. Ein auch auf Betreiben Deutschlands überarbeiteter Textentwurf verkürzte die Laufzeit für die Neugenehmigung und enthielt zusätzliche Auflagen. ${ }^{191}$ Zur finalen Vorlage der Kommission gab der Berufungsausschuss am 27. November 2017 die entscheidende positive Stellungnahme zugunsten einer erneuten Zulassung unter veränderten Bedingungen ab. ${ }^{192}$ Entsprechend dieser Stellungnahme beschloss die Kommission am 12. Dezem-

188 FAO/WHO, Pesticide residues in food 2016, Joint FAO/WHO Meeting on Pesticide Residues, Report of the special session of the Joint Meeting of the FAO Panel of Experts on Pesticide Residues in Food and the Environment and the WHO Core Assessment Group on Pesticide Residues Geneva, Switzerland, 9-13 May 2016, FAO Plant Production and Protection Paper 2016/227, S. 29 ff., 24, abrufbar unter: www.who.int (15.06.2019).

189 Mitteilung der Kommission vom 12.12.2017, C(2017) 8414 final, insbes. S. 8 f.

190 Erwägungsgrund 25 der Durchführungsverordnung (EU) 2017/2324 zur Erneuerung der Zulassung des Wirkstoffs Glyphosat.

191 S.u. C I.3.b) aa).

192 S.o. B I.2.b). 
ber 2017 gemäß Art. 20 VO (EG) 1107/2009 die Durchführungsverordnung (EU) 2017/2324 zur Ernenerung der Zulassung des Wirkstoffs Glyphosat. ${ }^{193}$ Im letzten Akt wurde die Liste der in der EU zugelassenen Wirkstoffe entsprechend aktualisiert. ${ }^{194}$

\section{b) Inhalt der erneuerten Genehmigung}

aa) Durchführungsverordnung Glyphosat

Soweit es den Inhalt der erneuten Genehmigung betrifft, springt zunächst die von ursprünglich zehn auf fünf Jahre verkürzte Laufzeit ins Auge. Die neue Genehmigung für Glyphosat gilt vom 16. Dezember 2017 bis zum 15. Dezember 2022 (Anhang I DurchführungsVO). Die Kommission erklärt dies damit, dass „im Vergleich zu anderen Wirkstoffen außerordentlich viele zusätzliche Informationen über Glyphosat veröffentlicht" werden. Dadurch solle den sich rasch vollziehenden künftigen Entwicklungen in Wissenschaft und Technik Rechnung getragen werden, aber auch dem Umstand, dass Glyphosat „eines der am häufigsten eingesetzten Herbizide in der Union“ sei (Erwägungsgrund $19 \mathrm{f}$. DurchführungsVO). Das klingt nicht so, als ob man fest von der Dauerhaftigkeit der gerade festgestellten Unbedenklichkeit von Glyphosat überzeugt war. Eine nur zwei- oder dreijährigen Verlängerung kam als Risikomanagement allerdings nicht in Betracht, weil das Peer Review-Verfahren bei der Wirkstoffzulassung einschließlich der obligatorischen Neuzulassung sämtlicher Pflanzenschutzmittel derartig zeitaufwändig ist, dass auch ein sofortiger Antrag für die nächste Verlängerung schon fast zu spät kommen würde. Somit besteht ein im Verfahren angelegtes Dilemma darin, dass das mehrstufige komplexe Prüfverfahren die Sicherheit nicht nur erhöht, sondern auch Druck in Richtung auf eine noch hinreichend lange Genehmigungslaufzeit erzeugt.

Soweit es die übrigen Bedingungen betrifft, geht die finale Fassung des Textes der Durchführungsverordnung zur erneuten Genehmigung von Glyphosat gerade auch auf deutsche Bemühungen zurück, die Wiedergenehmigung durch Änderungen zugunsten eines verstärkten Schutzes von Amateuranwendern und der biologischen

193 Fn. 74.

194 Durchführungsverordnung (EU) Nr. 540/2011 der Kommission vom 25. Mai 2011 zur Durchführung der Verordnung (EG) Nr. 1107/2009 des Europäischen Parlaments und des Rates hinsichtlich der Liste zugelassener Wirkstoffe, ABl. (EU) L 153 v. 11.06.2011, S. 1. Gemäß Art. 20 Abs. 3 i.V.m. Art. 13 Abs. 4 VO (EG) 1107/2009 i.V.m. Art. 2 DurchführungsVO (EU) 2017/2324 wurde der Anhang der DurchführungsVO (EU) 540/2011 aktualisiert. 
Vielfalt zu retten. ${ }^{195}$ Die Durchführungsverordnung lässt allerdings nicht schon auf den ersten Blick erkennen, welche Auflagen und Einschränkungen für die Glyphosatnutzung nun eigentlich gelten. Denn sie verweist auf die „Schlussfolgerungen des Überprüfungsberichts/Beurteilungsberichts zu Glyphosat und insbesondere dessen Anlagen I und II“, die „[b]ei der Anwendung der einheitlichen Grundsätze gemäß Artikel 29 Absatz 6 der Verordnung (EG) Nr. 1107/2009 ... zu berücksichtigen“ seien. Damit wird der Öffentlichkeit, die speziell an dieser Stelle auf eine transparente Darstellung angewiesen ist, der schnelle Zugriff auf wesentliche Informationen versperrt. Obwohl die neue Durchführungsverordnung 16 Anmerkungen mit Nachweisen enthält, sucht man nach einer Fundstelle für den zentral in Bezug genommenen Überprüfungsbericht/ Beurteilungsbericht der Kommission vergebens. Und obwohl Art. 18 VO (EU) 1141/2010 verlangt, diesen Bericht mit Ausnahme der vertraulichen Teile der Öffentlichkeit zugänglich zu machen, ist nur eine englische Version auffindbar. ${ }^{196}$

Führt man die Sonderbestimmungen aus Anhang I der Durchführungsverordnung (EU) 2017/2324 zur Erneuerung der Genehmigung für Glyphosat mit dem finalen Beurteilungsbericht der Kommission zusammen, auf den verwiesen wird und der seinerseits wieder auf andere Dokumente weiterverweist, ergeben sich folgende Einschränkungen und Modalitäten für die weitere Glyphosatnutzung:

- Glyphosat darf nur als Herbizid verwendet werden.

- Bei der obligatorischen Neuzulassung aller glyphosathaltigen Pflanzenschutzmittel müssen die Mitgliedstaaten bei der Anwendung der „einheitlichen Grundsätze“ gemäß Art. 29 Abs. 6 VO (EG) 1107/2009197 die Schlussfolgerungen des Überprüfungsberichts (Beurteilungsberichts) und dessen Anlagen I und II berücksichtigen - hierbei handelt es sich um besagte Verweisung.

Bei dieser Gesamtbewertung „achten“ die Mitgliedstaaten insbesondere auf folgende Belange, nämlich auf

- den „Grundwasserschutz in gefährdeten Gebieten“,

- den Schutz gewerblicher und nichtgewerblicher Verwender,

195 Kommission - Vertretung in Deutschland, Fragen und Antworten zur Erneuerung der Zulassung des Herbizids Glyphosat vom 28.11.2017: „Wir können bestätigen, dass ein [sic] Deutschland beantragt hat, die Anhänge des Entwurfs der Durchführungsverordnung dahingehend zu ändern, dass die Mitgliedstaaten bei der Bewertung von glyphosathaltigen Pflanzenschutzmitteln besonders auf den Schutz von Amateuranwendern und die Auswirkungen von Glyphosat auf die biologische Vielfalt achten müssen. Die Kommission schlug eine geänderte Fassung des Verordnungsentwurfs vor, um diesen Forderungen nachzukommen. Die Mitgliedstaaten stimmten den Änderungen zu“, abrufbar unter: https:// ec.europa.eu/germany/news/20171127GlyphosatFragenAntworten_de (15.06.2019).

196 European Commission (Directorate-General for Health and Safety), Final Review Report for the active substance glyphosate finalised in the Standing Committee on Plants, Animals, Food and Feed, SANTE/10441/2017 Rev 2 vom 09.11.2017.

197 D.h. insbesondere die Durchführungsverordnung (EU) Nr. 546/2011. S.o. C I.2.c). 
- das „Risiko für Landwirbeltiere und nicht zu den Zielgruppen gehörende terrestrische Pflanzen“,

- die „Bedrohung der Vielfalt und Abundanz [Anzahl von Organismen pro Flächenoder Raumeinheit] von Nichtziel-Landarthropoden [Gliederfüßer wie z.B. Insekten und Spinnentiere] und -Landwirbeltieren durch trophische Wechselwirkungen" sowie

- die Einhaltung der guten landwirtschaftlichen Praxis bei der Verwendung vor der Ernte (Anlage I DurchführungsVO).

Die Belange „Schutz gewerblicher und nichtgewerblicher Verwender" sowie „Bedrohung der Vielfalt" sind erst nachträglich im Berufungsausschuss, d.h. im Kontext der maßgeblichen Entscheidung vom 27. November 2017, eingefügt worden. ${ }^{198} \mathrm{Im}$ Hinweis auf den Schutz „nichtgewerblicher Verwender" ist die vieldiskutierte Frage der Verwendung glyphosathaltiger Mittel durch „Amateure“ angesprochen. Von der gerade auch vom deutschen Landwirtschaftsminister bekundeten Einschränkung des privaten Gebrauchs kann hier allerdings keine Rede sein. Es bleibt nämlich - wie bislang auch - den Mitgliedstaaten überlassen, wie sie Gesundheitsschäden bei Amateuranwendern ausschließen. Für diesen Belang gilt wie für alle anderen, dass die Anwendungsbedingungen „gegebenenfalls Maßnahmen zur Risikobegrenzung umfassen" müssen. Fraglich ist sogar, ob einzelne Mitgliedstaaten den privaten Gebrauch überhaupt verbieten dürfen. ${ }^{199}$

Der Überprüfungsbericht der Kommission enthält in seiner Schlussfolgerung („overall conclusion“) Referenzwerte über höchstzulässige Glyphosatmengen im menschlichen Körper, wodurch die Anforderung, auf den Schutz gewerblicher und nichtgewerblicher Verwender „zu achten“ doch noch etwas geschärft wird. Danach gelten sowohl als noch akzeptable tägliche Aufnahmemenge wie auch als ,akute Referenzdosis" jeweils 0,5 mg Glyphosat pro Kilogramm Körpergewicht. ${ }^{200}$ Wie die Kommission dazu erläutert, habe ihre Überprüfung zwar „akzeptable Expositionsszenarios" für Anwender, zufällig Anwesende und Wohnbevölkerung identifiziert. Dies müsse aber für jedes einzelne Pflanzenschutzmittel noch einmal im Einklang mit den einheitlichen Grundsätzen (Art. 29 Abs. 6 VO [EG] 1107/2009) „,bestätigt“ werden. ${ }^{201}$ Damit verweist die Kommission die Verantwortung im Wesentlichen an die Mitgliedstaaten.

Weiter heißt es in der DurchführungsVO,

- die Mitgliedstaaten „stellen sicher“, dass die Verwendung von Glyphosat in den in Art. 12 lit. a Richtlinie 2009/128/EG bestimmten Gebieten „minimiert wird“.

Hinter dieser Beschreibung verbergen sich „Gebiete, die von der Allgemeinheit oder von gefährdeten Personengruppen im Sinne von Artikel 3 der Verordnung (EG)

198 Überprüfungsbericht (Final Review Report), (Fn. 196), S. 7.

199 Dazu noch u. C II.2.c).

200 Überprüfungsbericht (Final Review Report), (Fn. 196), S. 4 f.

201 Ibid., S. 5. 
Nr. 1107/2009 genutzt werden, wie öffentliche Parks und Gärten, Sport- und Freizeitplätze, Schulgelände und Kinderspielplätze sowie Gebiete in unmittelbarer Nähe von Einrichtungen des Gesundheitswesens“. Das heißt: Das Versprühen glyphosathaltiger Pflanzenschutzmittel auf Flächen, bei denen wie im Falle der Sportplätze unmittelbarer Hautkontakt vorprogrammiert ist oder wo sich wie im Falle von Spielplätzen und Gesundheitseinrichtungen besonders verletzliche Personengruppen aufhalten, ist nicht etwa ausgeschlossen, sondern muss nur „minimiert“ werden - in welchem Umfang bleibt offen. ${ }^{202}$

Ferner

- gewährleisten die Mitgliedstaaten die Gleichwertigkeit der Spezifikationen des Materials, das gewerbsmäßig hergestellt wird, mit dem Testmaterial, das in den toxikologischen Studien verwendet wird.

Hinter dieser formal anmutenden Anforderung verbirgt sich eine sogenannte „critical area of concern“, nämlich der Umstand, dass acht der 24 antragstellenden Unternehmen im Glyphosat-Wiedergenehmigungsverfahren Spezifikationen präsentiert hatten, die nicht durch die toxikologische Bewertung gestützt waren. ${ }^{203}$ Ohne zu klären, ob sich diese Verfälschungen auf das Bewertungsergebnis im Wirkstoff-Neugenehmigungsverfahren ausgewirkt haben könnten, verschiebt die Kommission die gesamte Angelegenheit kurzerhand in die Pflanzenschutzmittel-Neuzulassung, d.h. zu den Mitgliedstaaten. Deshalb stellt sich die Frage, welchen Sinn das Erfordernis der Präsentation von Spezifikationen mit „korrespondierenden“ Studienergebnissen im Wirkstoff-Genehmigungsverfahren überhaupt noch hat.

Einen weiteren Problempunkt sieht der Überprüfungsbericht darin, dass einige Studien zu Glyphosat-Formulierungen positive Resultate zur Gentoxizität präsentiert hatten. Zwar waren diese Studien alle als nicht aussagekräftig oder sonst irrelevant bewertet worden. Die Kommission, die in der Genehmigungsverordnung auch hierauf Bezug nimmt, mahnt aber gleichwohl an, dass

- die Mitgliedstaaten sich bei der Neuzulassung der einzelnen Pflanzenschutzmittel mit deren gentoxischen Potenzial befassen müssen. ${ }^{204}$

Eine solche Entscheidung macht Sinn, wenn feststeht, dass die Gentoxizität sicher nicht mit Glyphosat, sondern nur mit bestimmten Anwendungsprodukten aufgrund der jeweils verwandten Beistoffe assoziiert ist. Davon kann aber keine Rede sein, so dass sich die Kommission auch hier der Verantwortung entzieht.

Die Neugenehmigung verweist schließlich auch vermittels des Überprüfungsberichts der Kommission auf die sogenannten

$202 \mathrm{Zu}$ den Möglichkeiten der Mitgliedstaaten noch u. C II.2.c).

203 Überprüfungsbericht (Final Review Report), (Fn. 196), S. 5; EFSA-Schlussfolgerung, (Fn. 1), S. 25.

204 Überprüfungsbericht (Final Review Report), (Fn. 196), S. 6 f. 
- „Endpoints“ aus der EFSA-Schlussfolgerung, die einer angemessenen Anwendung von Art. 29 Abs. 1 VO (EG) 1107/2009 (allgemeine Anforderungen für die Zulassung zum Inverkehrbringen von Pflanzenschutzmitteln) und der einheitlichen Grundsätze aus VO (EU) 546/2011 dienen sollen.

Die Endpoints finden sich in Anhang A der EFSA-Schlussfolgerung und teilweise auch in den Anhängen I und II des Überprüfungsberichts der Kommission. Dabei geht es im Wesentlichen um chemische Daten und Grenzwerte, u.a. Anforderungen an den Reinheitsgrad des Wirkstoffes, Höchstmengen der Verwendung des für die Glyphosat-Wiedergenehmigung repräsentativ herangezogenen Pflanzenschutzmittels „MON52276“, Grenzwerte für Rückstände von Glyphosat bzw. AMPA in Lebens- und Futtermitteln $(0,05 \mathrm{mg} / \mathrm{kg}$ bei solchen pflanzlicher Art; $0,025 \mathrm{mg} / \mathrm{kg}$ in Fleisch, Milch und Eiern; $0,05 \mathrm{mg} / \mathrm{kg}$ in Leber, Nieren und Fett), im Boden $(0,05 \mathrm{mg} /$ $\mathrm{kg}$ ), im Trink- und Oberflächenwasser (0,03 Mikrogramm/Liter) oder in der Luft (5 Mikrogramm $/ \mathrm{m}^{3}$ ), toxische Glyphosatmengen, Zerfallszeiträume, Potential zur Anreicherung in verschiedenen Fleischarten (keines), maximale Rückstandsmengen in den verschiedenen Agrarprodukten, Abbaugeschwindigkeiten, aber auch Anforderungen an die Analyse-Methoden. ${ }^{205}$ Der besonders wichtige Höchstwert für die für Menschen noch akzeptable Tagesdosis an Glyphosat (Acceptable Daily Intake [ADIWert]) wurde auf 0,5 mg/kg Körpergewicht festgesetzt, ebenso die akute Referenzdosis (acute reference dose [ARfD]). ${ }^{206}$

Ein klares Verbot enthält die DurchführungsVO nur an einer Stelle:

- Die Mitgliedstaaten stellen sicher, dass der Beistoff „POE-Tallowin“ nicht weiter in glyphosathaltigen Anwendungsprodukten enthalten ist.

Dieser Regelung dürfte aber kaum mehr praktische Bedeutung zukommen, weil sie in etlichen Staaten (z.B. Deutschland) längst umgesetzt ist. ${ }^{207}$ Somit ergibt sich insgesamt, dass die vermeintlichen Einschränkungen gerade auch in den kritischen Bereichen darin bestehen, den Mitgliedstaaten allgemein gehaltene Beachtenspflichten bei der Pflanzenschutzmittel-Neuzulassung aufzuerlegen, so dass sich die europäische Ebene ihrer Verantwortung entzieht. ${ }^{208}$

205 EFSA-Schlussfolgerung, (Fn. 1), Anhang A (S. 30 ff.).

206 Ibid., Anhang A (S. 41): ARfD bezeichnet die Substanzmenge pro kg Körpergewicht, die über die Nahrung mit einer Mahlzeit oder innerhalb eines Tages ohne erkennbares Risiko aufgenommen werden kann.

207 In Deutschland z.B. ordnete das BVL schon im Jahr 2009 den Austausch des Netzmittels POE-Tallowamin bis 2010 an und verbot im Januar 2010 die Verwendung von Futtermitteln, bei deren Anbau in Deutschland Spritzmittel mit Tallowaminen eingesetzt wurden. Dieses Verbot ist inzwischen aufgehoben, vermutlich weil das Netzmittel nicht mehr beigegeben wird. Siehe dazu BT-Drs. 17/14291 vom 01.07.2013, S. 1.

208 Kritisch hierzu auch die Entscheidung der Europäischen Ombudsfrau O'Reilly vom 18.02.2016 im Fall 12/2013/MDC on the practices of the European Commission regarding the authorisation and placing on the market of plant protection products (pesticides), insbes. $\int \mathbb{S} 43$ f., 48,63 . 
bb) Folgeentscheidungen: Erlaubte Rückstände von Glyphosat in Lebens- und Futtermitteln

Gemäß Art. 12 der Verordnung (EG) 396/2005 über Höchstgehalte an Pestizidrückständen in oder auf Lebens- und Futtermitteln ${ }^{209}$ unterbreitet EFSA der Kommission und den Mitgliedstaaten innerhalb von zwölf Monaten ab dem Zeitpunkt der Aufnahme eines Wirkstoffs in Anhang I der Richtlinie 91/414/EWG (inzwischen: DurchführungsVO [EU] 540/2011) eine Stellungnahme über die ihrer Auffassung nach angemessenen Rückstandshöchstwerte für Glyphosat in den einzelnen Lebens- und Futtermitteln oder über erforderliche Änderungen. Schon in der Vergangenheit waren die Rückstandswerte u.a. auf Betreiben von Monsanto zum Teil erheblich hochgesetzt worden. So genehmigte EFSA Anfang 2012 eine Anhebung des maximalen Glyphosatgehalts in Linsen um das 100-fache (von $0.1 \mathrm{mg} / \mathrm{kg}$ auf $10 \mathrm{mg} / \mathrm{kg}$ ), um den Import von Linsen aus den USA und Canada zu ermöglichen, die dort erlaubterweise zur Sikkation mit Glyphosat behandelt wurden. ${ }^{210}$ Die neueste Stellungnahme ("Reasoned Opinion") der EFSA stammt von 2018. ${ }^{211}$ Aus der umfangreiche Liste ergeben sich zahlreiche Änderungsvorschläge, die sowohl Verminderungen wie auch Ausweitungen der Höchstrückstandswerte (Maximum Residue Levels [MRLs]) für Glyphosat enthalten. Vorgeschlagen wird u.a. die Zurücksetzung des Wertes für wilde Pilze von 50 (!) auf 0,05 mg/kg, die Erhöhung für die meisten Getreidekörner von 10 bzw. 20 auf $30 \mathrm{mg} / \mathrm{kg}$, für Kuhmilch von 0,05 auf 0,1 mg/kg oder für Linsen erneut von 10 auf $15 \mathrm{mg} / \mathrm{kg}$. EFSA betont, dass die toxikologischen Daten in der vorliegenden Überprüfung nicht berücksichtigt seien und mahnt im Zusammenhang mit etlichen Vorschlägen weiteren Forschungsbedarf an. ${ }^{212}$ Man muss im Hinblick darauf wie auch im Hinblick auf die drastischen Schwankungen der Werte davon ausgehen, dass diese eher nicht wissenschaftlich fundiert sind.

\section{c) Konnte nur durch die Zustimmung Deutschlands der Glyphosatverbrauch eingeschränkt werden?}

Wie erwähnt, hatte Bundeslandwirtschaftsminister Schmidt (CSU) im ACoPAFF die entscheidende Stimme für die Annahme der positiven Stellungnahme abgeben lassen. Dies erklärte er damit, dass er nur durch seine Zustimmung habe erreichen können, den Glyphosat-Einsatz „für privaten Gebrauch und für andere Gebräuche zu redu-

209 Verordnung (EG) Nr. 396/2005 des Europäischen Parlaments und des Rates vom 23. Februar 2005 über Höchstgehalte an Pestizidrückständen in oder auf Lebens- und Futtermitteln pflanzlichen und tierischen Ursprungs und zur Änderung der Richtlinie 91/414/ EWG des Rates, ABl. (EU) L 70 v. 16.03.2005, S. 1.

210 EFSA, Peer Review Report vom 16.08.2017, S. 518, unter Hinweis auf die "Reasoned Opinion" der EFSA vom 16.01.2012 ("Modification of the existing MRL for glyphosate in lentils”), abrufbar unter: www.efsa.europa.eu/en/search/doc/2550.pdf (15.06.2019).

$211 E F S A$, Review of the existing maximum residue levels for glyphosate according to Article 12 of Regulation (EC) No 396/2005, Reasoned Opinion, approved on 17 April 2018, doi: 10.2903, EFSA Journal 2018/16, S. 5263, abrufbar unter: https://efsa.onlinelibrary.wiley. com (15.06.2019).

212 Ibid., S. 3 sowie Liste im Anhang. 
zieren“. ${ }^{213}$ Wie bereits gezeigt wurde, trifft diese Aussage schon deshalb nicht zu, weil der neuen Genehmigungsverordnung insoweit keine harten Verpflichtungen zu entnehmen sind. ${ }^{214}$ Trotzdem soll mit Blick auf künftige Fälle gefragt werden, was geschehen wäre, wenn sich der deutsche Vertreter im ACoPAFF enthalten hätte, wie es den Regeln der heimischen Koalitionsregierung ${ }^{215}$ eigentlich entsprochen hätte. Könnte die Kommission nötigenfalls selbst über die Erneuerung einer Genehmigung entscheiden? Hierzu müssen die europäischen Regeln für einen Entscheidungsausfall auf Seiten der Mitgliedstaaten herangezogen werden.

Für Erneuerungsverordnungen zur Wiedergenehmigung eines Wirkstoffs gilt gemäß Art. 20 Abs. 1 VO [EG] 1107/2009 das Ausschuss- oder Komitologieverfahren gemäß Art. 79 Abs. 3 VO [EG] 1107/2009. Diese Norm verweist auf die Artikel 5 und 7 des inzwischen aufgehobenen Beschlusses 1999/468/EG. Was heute anstelle dieser Artikel gilt, ist in den Übergangsbestimmungen der neuen Komitologie-Verordnung (EU) 182/2011216 geregelt. Gemäß Art. 13 Abs. 1 lit. c VO (EU) 182/2011 findet im Falle der Verweisung auf Art. 5 des obsoleten Beschlusses 1999/468/EG das in Art. 5 VO (EU) 182/2011 genannte Prüfverfahren Anwendung „und es wird davon ausgegangen, dass der Basisrechtsakt vorsieht, dass die Kommission, wie in Artikel 5 Absatz 4 Unterabsatz 2 Buchstabe b vorgesehen, den im Entwurf vorgesehenen Durchführungsrechtsakt ohne Stellungnahme nicht erlassen darf“. Es wird also fingiert, dass der Basisrechtsakt - d.h. die Pflanzenschutzmittel-VO (EU) 182/2011 - auf Art. 5 Abs. 4 UAbs. 2 lit. b VO (EU) 182/2011 verweist, mit der Folge, dass die Kommission den Durchführungsakt nicht selbst erlassen kann, wenn die Mitgliedstaaten - wie hier im Ständigen Ausschuss (SCoPAFF) - keine Stellungnahme zustande bringen. Sie dürfte ihn allenfalls im Notfall erlassen, wenn er nämlich „unverzüglich erlassen werden muss, um eine erhebliche Störung der Agrarmärkte oder eine Gefährdung der finanziellen Interessen der Union" abzuwenden (Art. 5 Abs. 4 Unterabs. 2 i.V.m. Art. 7 VO [EU] 182/2011). Eine solche Situation lag hier aber schon deshalb nicht vor, weil zusätzliche Prüfungen durchgeführt und die Fristen für einzelne Verfahrensabschnitte bis zur Genehmigung mehrfach verlängert worden waren. Wäre es also nicht zur Einschaltung des Berufungsausschusses SCoPAFF gekommen, wäre die Erneuerung der Wirkstoffgenehmigung für Glyphosat gescheitert.

Der Kommission blieb aber die Möglichkeit, gemäß Art. 5 Abs. 4 UAbs. 3 VO (EU) 182/2011 den Berufungsausschuss ACoPAFF fristgerecht zu befassen. Wäre auch hier keine qualifizierte Mehrheit für oder gegen den Verordnungsentwurf der Kommission erreicht worden, wäre Art. 6 Abs. 3 Unterabs. 2 VO (EU) 182/2011 zur Anwendung gekommen, wo es heißt: „Wird keine Stellungnahme abgegeben, so kann die Kom- 
mission den im Entwurf vorgesehenen Durchführungsrechtsakt erlassen. “217 Das heißt, im Berufungsverfahren (ACoPAFF) ist die Kommission anders als im Grundverfahren (ScoPAFF) nicht mehr gehindert, die Durchführungsverordnung zur Genehmigung auch ohne die Mitgliedstaaten zu erlassen. Art. 6 Abs. 3 UAbs. 2 VO (EU) 182/2011 stellt dabei klar, dass sie handeln „kann“, nicht muss. Da die Kommission die Erneuerung der Glyphosatgenehmigung wollte, ${ }^{218}$ hätte sie die Durchführungsverordnung sehr wahrscheinlich selbst erlassen. Eine solche Lösung wollte die Kommission aber gerade vermeiden, weil sie dann die Mitgliedstaaten aus ihrer Verantwortung für eine erneute Glyphosatzulassung entlassen und diese letztlich allein übernommen hätte. Deshalb hatte der zuständige EU-Gesundheitskommissar die Mitgliedstaaten unmissverständlich ermahnt, sich nicht weiter hinter der Kommission zu verstecken. ${ }^{219}$ Hätte die Kommission allein handeln müssen, hätte die Kommission zwar nur den „im Entwurf vorgesehenen Durchführungsrechtsakt“ erlassen dürfen (Art. 6 Abs. 3 UAbs. 2 VO [EU] 182/2011). Zugleich ergeben sich aber Verhandlungsoptionen für alle Beteiligten daraus, dass der Entwurf im Berufungsverfahren noch umgestaltet werden kann, was gerade auch im Glyphosatverfahren geschah. ${ }^{220}$ Gemäß Art. 6 Abs. 2 VO (EU) 182/2011 kann jedes Mitglied des Berufungsausschusses Änderungen am Entwurf des Durchführungsrechtsakts vorschlagen und die Kommission entsprechende Änderungen beschließen oder ablehnen, wobei sie sich um Lösungen bemühen muss, die möglichst breite Unterstützung finden. Angesichts der Weite dieser Formulierung, dürfte insoweit ein erheblicher Einschätzungsspielraum der Kommission bestehen. Welche mitgliedstaatlichen Vorschläge die Kommission im Interesse einer breiten Unterstützung aufgreift, hängt zwar grundsätzlich nicht von der Zustimmung eines einzelnen Mitgliedstaats ab. Denkbar ist aber, dass ein großer Mitgliedstaat wie z.B. Deutschland dem Vorsitz (der Kommission) seine Zustimmung (nur) für den Fall in Aussicht stellt, dass dieser seinen Änderungsvorschlag übernimmt. Wenn die Stellungnahme der Mitgliedstaaten ohne diesen einen Mitgliedstaat nicht zustande kommen kann, könnte die Kommission durchaus geneigt sein, auf den vorgeschlagenen Handel einzugehen. Im Glyphosatverfahren kam es, wie gezeigt, aber nicht zur Aufnahme einer Klausel über die obligatorische Reduzierung des Glyphosatverbrauchs durch Amateure, sondern zu einer Formulierung, die den Mitgliedstaaten lediglich aufgibt, auf den "Schutz“ der nichtprofessionellen Anwender zu „achten“. Ob Deutschland Härteres im Berufungsausschuss gefordert hatte, kann hier nicht beurteilt werden. In jedem Falle hat der deutsche Minister die Verabschiedung von Klauseln erreicht, die es ohne harte Beschränkungen und letztlich ohne Mehrwert

217 Da sich die Fiktion in der Überleitungsvorschrift Art. 13 Abs. 1 lit. c VO (EU) 182/2011 ausdrücklich auf Art. 5 Abs. 4 Unterabs. 2 lit. b bezieht, soll sie offenbar nur den Fall der fehlenden Stellungnahme der Mitgliedstaaten im SCoPAFF-Verfahren (Art. 5), aber nicht im ACoPAFF-Verfahren (Art. 6) erfassen.

218 Siehe Erwägungsgrund 25 der DurchführungsVO (EU) 2017/2324 der Kommission.

219 Michalopoulos, in: EURACTIV.com vom 06.10.2017 (“Andriukaitis: 'Enough' with member states hiding behind the Commission on glyphosate”), abrufbar unter: www.euractiv .com (15.06.2019).

220 Alle Entwürfe sind auf der Seite der Kommission abrufbar unter: https://ec.europa.eu/fo od/plant/pesticides/glyphosate_en (15.06.2019). 
gegenüber der bestehenden Rechtslage 221 ermöglichten, die deutsche Zustimmung vor der Öffentlichkeit zu rechtfertigen.

\section{Der Streit um die Risikobewertung}

Die hier vorliegende juristische Studie ist nicht der Ort, um naturwissenschaftliche Streitigkeiten um die Risikobewertung zu entscheiden. Praktisch jede der glyphosatrelevanten Studien wird unabhängig davon, welche Seite ihr Ergebnis jeweils unterstützt, hinsichtlich der Angemessenheit der Versuchsanordnung, der verwendeten Methoden, der Repräsentativität der untersuchten Fälle oder der Bewertungen kritisiert. ${ }^{222}$ Unbestritten ist aber wohl, dass es sich bei den brisantesten Studien, die eine potentielle Kanzerogenität von Glyphosat annehmen, um statistikbasierte Meta-Analysen handelt, die schon ihrer Natur nach nicht dazu dienen, einen Kausalzusammenhang zu belegen, sondern allenfalls Hinweise auf einen möglichen Zusammenhang (z.B. auffällige Korrelationen) geben können. ${ }^{223}$ Deshalb stellt sich die Frage, wie relevant statistische Hinweise in isolierter Form sein können, ob sie also genügen, um ein Risiko im Zusammenhang mit Kanzerogenität oder Gentoxizität zu begründen. U.a. diese Frage war auch Gegenstand der hier sogenannten „Portier-Kontroverse“. Der Schwerpunkt der Streitigkeiten lag aber weniger im Bereich der Beachtung wissenschaftlicher Standards als vielmehr in Vorwürfen betreffend die mangelnde Objektivität und Unabhängigkeit der Bewertung, insbesondere durch EFSA und das deutsche BfR.

\section{a) Die Portier-Kontroverse}

In Reaktion auf die EFSA-Schlussfolgerung zum Peer Review vom Herbst 2015, die eine Kanzerogenität von Glyphosat für „unwahrscheinlich“ erklärte, ${ }^{224}$ bildete sich um den Wissenschaftler und früheren Direktor des US National Center for Environmental Health und der US Agency for Toxic Substances and Desease Registry, Christopher Portier, eine Gruppe von 98 Wissenschaftlern und Wissenschaftlerinnen aus aller Welt, die ihre „tiefe Besorgnis“ über diese Schlussfolgerung ausdrückten und ihr entschieden entgegentraten. Die überwiegend hochrangigen Experten und Expertinnen aus allen relevanten Teilbereichen der Medizin, Biologie, Pharmakologie, Epidemiologie, Statistik und Umwelttoxikologie hoben Ende November 2015 in einem offenen Brief ${ }^{225}$ an den europäischen Gesundheitskommissar Andrinkaitis hervor, dass die IARC eine hochrespektierte Fach-Institution der WHO im Bereich der Krebser-

221 S.o. C I.3.b) aa).

222 Siehe z.B. in Bezug auf die oben (C I.3.a) erwähnte Studie von Schinasi und Leon die Kritik von Chang/ Delzell, Journal of Environmental Science and Health 2016/51 Part B, S. 403.

223 Siehe z.B. Ibid., S. 403.

224 Fn. 1.

225 Portier (Corresponding Author) et al., Open Letter: Review of Carcinogenicity of Glphosate by EFSA and BfR, 27.11.2015, abrufbar auf der EFSA-Homepage unter: www.efsa.europa.eu/sites/default/files/Prof_Portier_ldetter.pdf (15.06.2019). 
krankungen sei, die nach umfassendem Peer Review während zwölfmonatiger Bewertung durch 17 unabhängige Experten zum gegenteiligen Schluss (wahrscheinliche Kanzerogenität von Glyphosat) gelangt sei. Im Vergleich zur Bewertung durch die EFSA und das deutsche BfR sei die Entscheidung der IARC „bei Weitem glaubwürdiger", weil sie auf einem offenen und transparenten Verfahren beruhe, das Vorkehrungen zur Sicherung der Unabhängigkeit der befassten Experten, insbesondere von den interessierten Unternehmen, getroffen habe. Dagegen sei die Entscheidung des BfR „nicht glaubwürdig“, weil sie diese Voraussetzungen nicht erfülle. Angesichts der Tatsache, dass selbst das BfR eine begrenzte Evidenz ("limited evidence") für eine kanzerogene Wirkung auf Menschen gesehen habe, sei es unangemessen, die Krebsgefahr für Menschen - wie EFSA - als „unwahrscheinlich“ zu bewerten.

Im Januar 2016 verteidigte der Geschäftsführende Direktor von EFSA die Bewertung seiner Behörde mit im Wesentlichen vier Argumenten: ${ }^{226}$ Erstens stelle die Screening-Bewertung der IARC nur einen ersten Schritt dar und könne nicht mit der „umfassenderen Risikobewertung“ der EFSA verglichen werden. Zweitens verwende EFSA ein anderes Einstufungssystem, das Gefährdungen durch jeden einzelnen chemischen Stoff oder Stoffverbindungen erkennen könne, während die IARC so unterschiedliche Gegenstände wie Glyphosat, rotes Fleisch oder stark gebratene Lebensmittel in dieselbe Kategorie „wahrscheinlich krebserregend“ einordne. Drittens verkenne die Kritik, dass EFSA „sich nur auf den Wirkstoff konzentrierte“, während die Mitgliedstaaten für die Pflanzenschutzmittel-Rezepturen verantwortlich seien. ${ }^{227}$ Und viertens habe EFSA alle Regeln einer transparenten, offenen und objektiven Bewertung eingehalten und von allen befassten Wissenschaftlern zum Teil auch über die Verpflichtungen hinaus „Declarations of Interest“ zur Sicherstellung der Unabhängigkeit verlangt.

Dies konnte die öffentliche Unruhe allerdings nicht beschwichtigen. Im März 2016 verlangten Mitglieder des Europäischen Parlaments öffentlichen Zugang zu den kompletten Aufzeichnungen über Tierlabordaten im Zusammenhang mit verwerteten Studien über die chronische Karzinogenität von Glyphosat. Die betreffenden Daten waren zum Teil als vertrauliche Geschäftsgeheimnisse eingestuft worden; zum Teil waren die Studien in einer Studie von Greim et al. (2015) dargestellt. Auf das Ersuchen der Parlamentarier hin entschied sich die Kommission, zumindest die Tabellen mit den Rohdaten freizugeben. ${ }^{228}$

226 Antwortschreiben der EFSA (Geschäftsführender Direktor B. Url) an C. Portier betreffend Offener Brief, 13.01.2016, abrufbar unter: www.efsa.europa.eu/de/press/news/1601 13 (15.06.2019).

227 Auf ihrer Homepage erklärt EFSA unter dem Stichwort „Glyphosat“ (Aktuelles), dass „die Vorwürfe auf einem Missverständnis des Peer Review-Verfahrens beruhten“, abrufbar unter: www.efsa.europa.eu/de/topics/topic/glyphosate (15.06.2019).

228 Siehe Pressemeldung der EFSA vom 16.12.2009 („Glyphosat: EFSA gibt Rohdaten aus Risikobewertung frei"), abrufbar unter: www.efsa.europa.eu/de/press/news/161209 (15.06.2019). Zur Studie von Greim et al., Critical Reviews in Toxicology 2015/45, S. 185 ff. 
Nach Prüfung der neu zugänglichen Daten versandte Christopher Portier im Mai 2017 einen weiteren Offenen Brief,229 diesmal an den Kommissionspräsidenten Juncker. Nun warf er EFSA und ECHA vor, nicht alle signifikanten Erkenntnisse betreffend die Krebsgefahr in ihre Bewertung einbezogen und die wissenschaftlichen Standards verletzt zu haben. Konkret beanstandete er, dass acht genauer bezeichnete tumorrelevante Studien nicht berücksichtigt worden seien. Das deutsche BfR wurde beschuldigt, von 21 positiven Tumorerkenntnissen nur etwa 20\% berücksichtigt zu haben.

Daraufhin beauftragte die Europäische Kommission ECHA und EFSA, sich mit den fraglichen Dokumentationslücken bzw. Mängeln auseinanderzusetzen. Die beiden Einrichtungen setzten sich dann zwar in einem „Technical Assessment“ ausführlich mit den Vorwürfen auseinander, beharrten aber auf die ordnungsgemäße Gewinnung und Richtigkeit ihrer Ergebnisse. ${ }^{230}$ Die Argumentation lief im Wesentlichen darauf hinaus, dass alle Studien, die Portier angeführt hatte, entweder an Unzulänglichkeiten (insbesondere betreffend die Testmethoden) litten, biologisch nicht relevant oder in ihren Ergebnissen statistisch nicht signifikant seien. EFSA gewichte und bewerte die statistische Signifikanz und die biologische Relevanz des karzinogenen Potentials von Glyphosat nach dem Ansatz der integrierten Nachweisgewichtung ("integrated weight of evidence approach"), der voll und ganz den Richtlinien entspreche. Allein statistische Hinweise auf ein erhöhtes Auftreten von Krebs genügten danach nicht. EFSA räumte zwar ein, dass 7 der 8 Tumorbefunde weder im Renewal Assessment Report (RAR) des BfR von 2015 noch im CLH (harmonized classification and labelling) Report des EFSA Committee for Risk Assessment (RAC) von 2016 dokumentiert worden waren. Das heiße aber keineswegs, dass sie übersehen oder weggelassen, sondern nur, dass sie als nicht gefahren- und bewertungsrelevant ("not relevant for hazard and risk assessment") angesehen worden seien. Auch den Vorwurf mangelnder Sorgfalt in Bezug auf das EFSA Committee for Risk Assessment weise man entscheiden zurück. Man verstehe, dass es unterschiedliche Auffassungen über das Ausmaß an Detail ("level of detail") geben könne, das sich in den Bewertungsberichten niederschlagen sollte. Auch gäbe es unterschiedliche Meinungen zur Tauglichkeit bestimmter Testverfahren. Es bestehe aber insgesamt kein Anlass, die vorhandene Bewertung infrage zu stellen und den Bewertungsprozess, wie gefordert, zu wiederholen.

\section{b) Kritische Fragen zur Rolle des BfR im Deutschen Bundestag}

Im April 2016 hatten einige Abgeordnete sowie die Fraktion BÜNDNIS 90/DIE GRÜNEN im Bundestag anlässlich des laufenden EU-Genehmigungsverfahrens eine

229 Offenen Brief von Portier vom 28.05.2017 an den Kommissionspräsidenten Juncker, abrufbar beim Natural Resources Defense Council unter: www.nrdc.org/sites/default/files/ open-letter-from-dr-christopher-portier.pdf (15.06.2019).

230 Auch zum Folgenden das Antwortschreiben von ECHA und EFSA vom 05.07.2017, Ref. GDS/JVT/mc (2017) - out - 17997220, abrufbar bei EFSA unter: www.efsa.europa.eu/si tes/default/files/170706-glyphosate-letter.pdf (15.06.2019). 
Kleine Anfrage an die Bundesregierung zu Glyphosat gerichtet, u.a. auch zur Frage nach der Unabhängigkeit leitender Beamter im BfR. ${ }^{231}$ Dabei ging es insbesondere um Dr. R.S., den Leiter der Abteilung Sicherheit von Pestiziden im BfR. Dieser war gleichzeitig Leiter der „Expert Taskforce on Glyphosate“ der Joint FAO/WHO Meeting on Pesticides Residues (JPMPR), die nach dem Votum der IARC vom März 2015 („wahrscheinlich krebserregend für Menschen (Gruppe 2A)“232 zu dem gegenteiligen Ergebnis gekommen war, dass kein solches Krebsrisiko bestehe. An der Neubewertung durch das JPMPR hätten „nach Kenntnis der Bundesregierung“ keine Mitarbeiter des BfR oder anderer deutscher Behörden teilgenommen. Die Frage, ob Dr. R.S. früher „mit der Lobbyorganisation ILSI-HESI (International Life Sciences Institute - Health and Environmental Sciences Institute)“ zusammengearbeitet habe, bejahte die Bundesregierung jedoch, und zwar mit der Präzisierung, dass er einmalig an einem Workshop dieser Organisation betreffend Teststrategien der toxikologischen Bewertung teilgenommen habe und in diesem Zusammenhang auch als CoAutor einer Publikation aus dem Jahre 2006 aufgeführt sei. Die Frage, ob Dr. R.S. oder andere Mitarbeiter in die Bewertung bei der anstehenden Glyphosat-Genehmigungserneuerung involviert seien, wurde nicht konkret beantwortet. Die Bundesregierung verwies teils auf allgemeine Expertenlisten von FAO und WHO, teils berief sie sich auf den Schutz des Rechts dieser Personen auf informationelle Selbstbestimmung sowie darauf, dass Vertreter des BfR, die mit der Glyphosat-Bewertung befasst gewesen seien, Morddrohungen erhalten hätten, weshalb personenbezogene Daten aus Gründen der Fürsorgepflicht nicht mitgeteilt werden könnten. Die Frage, an welche von der „Glyphosate Task Force“ (Antragstellerin im Genehmigungsverfahren) beauftragte Consulting-Firma die verschiedenen Versionen des Glyphosate Renewal Assessment Report, insbesondere der revidierte RAR vom 2. Februar 2015, gelangt seien, wurde nicht beantwortet. Denn der Name der Consulting-Firma sei eine vertrauliche Information, die dem Geschäfts- und Betriebsgeheimnis gemäß Art. 63 Abs. 2 lit. e VO (EG) 1107/2009 unterliege. Und wegen der Kürze der Zeit zur Beantwortung der insgesamt 73 Fragen habe die Bundesregierung auch nicht die Zustimmung der Mitgliedsfirmen der Glyphosate Task Force einholen können. Demgegenüber räumte sie eine Zusammenarbeit u.a. mit der „genius GmbH“, Darmstadt, einer Consultingfirma, ein, die bis heute den Außenauftritt der „Glyphosate Task Force“ betreut. ${ }^{233}$ Die fünf Aufträge, die das BfR zwischen 2007 und 2012 an die genius $\mathrm{GmbH}$ vergeben haben soll, umfassen Moderationen bei wichtigen Veranstaltungen (z.B. „Grüne Woche“), aber auch eine interne Schulung für Nachwuchswissenschaftler im JuliusKühn-Institut (Bundesforschungsinstitut für Kulturpflanzen), das in die Risikobewertung von Pflanzenschutzmitteln mit eingeschaltet ist ( $\mathbb{5 7}$ PflSchG).

231 S. auch zum Folgenden die Antwort der Bundesregierung, (Fn. 37), BT-Drs. 18/8408, insbes. Vorbemerkungen und Fragen 7, 12, 14, $18 \mathrm{f}$.

232 S.o. A II.

233 Siehe Peer Review Report, (Fn. 210), S. 385, sowie das Impressum des Informationsportals der „Glyphosate Task Force“, Stand: 10.01.2019, abrufbar unter: https://www.glyphosat .de/impressum (15.06.2019). 
Unmittelbar nach der entscheidenden Abstimmung über die erneute GlyphosatGenehmigung richtete im Dezember 2017 eine Reihe von Abgeordneten aus verschiedenen Fraktionen des Bundestages schriftliche Anfragen an die Bundesregierung, die vom Bundesministerium für Ernährung und Landwirtschaft (BMEL) beantwortet wurden. ${ }^{234}$ Die Frage nach Kontakten des zuständigen Fachreferats, aber auch des Bundeskanzleramts mit der Antragstellerin im Genehmigungsverfahren, der Unternehmensgruppe "Glyphosate Task Force“, wurde dahingehend beantwortet, dass das Bundeskanzleramt im Jahr 2017 keine Kontakte gehabt habe, unterhalb der Leitungsebene aber mit „Verbänden und Fachkreisen“ bzw. „Unternehmen und Verbänden, darunter auch Nichtregierungsorganisationen“, wenn diese von Gesetz- und Verordnungsentwürfen betroffen seien, „kontinuierlich Gespräche“ geführt worden seien. Das sei aufgabenbedingt. Dabei listete das BMEL sieben Veranstaltungen und Fachgespräche auf und berief sich im Übrigen darauf, dass eine „vollständige und umfassende Aufstellung ... aus den zuvor genannten Gründen nicht erfolgen“ könne. ${ }^{235}$ Welche Gründe das sind, bleibt unklar.

Im September 2017 hatten die italienische Zeitung „La Stampa“ und der britische „Guardian" aufgedeckt, dass rund 100 Seiten des 4.300 Seiten umfassenden Schlussberichts der EFSA (betreffend den Renewal Assessment Report [RAR] des BfR) von 2015 teilweise wortwörtlich aus einem Monsanto-Papier übernommen worden waren. ${ }^{236}$ Dieses Papier hatte Monsanto 2012 als federführendes Unternehmen im Namen der ca. 20 Unternehmen umfassenden "Glyphosate Task Force“ (Antragsteller) als Begleitbericht zum Antrag auf Erneuerung der Glyphosat-Genehmigung eingereicht. Wie die genannten Zeitungen durch Textgegenüberstellungen zeigten, umfassten die betreffenden Passagen auch brisante Analysen, so etwa den Versuch des früheren Monsanto-Angestellten John Acquavella und der seinerzeitigen MonsantoAngestellten Donna Farmer, einen Zusammenhang zwischen der Verwendung glyphosathaltiger Mittel und dem Non-Hodgkin-Lymphom mit Hinweis auf Schwächen der betreffenden Studie zu bestreiten. Inzwischen hat ein Plagiatsforscher in KoAutorschaft mit einem Biochemiker und Anti-Glyphosat-Aktivisten eine Studie vorgelegt, die im Wege der statistischen Analyse und Gegenüberstellung von Texten des BfR und der Glyphosate Task Force aufzeigt, dass der Anteil der plagiierten Teile noch erheblich größer als ursprünglich angenommen ist. Die Studie von 2019,237 die sorgfältig zwischen „guten“ (arbeitserleichternden) und „schlechten“ (plagiierenden) Copy-and-paste-Aktionen unterscheidet, belegt ein erschütterndes Ausmaß an Textstellen, deren eigentliche Quellen nicht offengelegt sind, d.h. von Plagiaten. Sie gelangt zu dem Schluss, dass die Bewertung nicht unabhängig durchgeführt worden sei und

234 Auch zum Folgenden BT-Drs. 19/280 vom 15.12.2017, S. 45-52.

235 BT-Drs. 19/280, S. 47.

236 Auch zum Folgenden Neslen, in The Guardian vom 15.09.2017 ("EU report on weedkiller safety copied text from Monsanto study"), abrufbar unter: www.theguardian.com (15.06.2019).

237 Weber/Burtscher-Schaden, Detailed Expert Report on Plagiarism and superordinated Copy Paste in the Renewal Assessment Report (RAR) on Glyphosate, Januar 2019, abrufbar auf der Seite der EP-Fraktion der Grünen unter: www.greens-efa.eu/files/doc/docs/298f f6ed5d6a686ec799e641082cdb63.pdf (15.06.2019). 
sich dies auch auf deren Qualität insbesondere in puncto Gesundheitsrisiken ausgewirkt habe.

EFSA versicherte auf die Vorwürfe hin, ihre Bewertung wie auch die zugrunde liegende Bewertung durch das deutsche BfR sei in einem korrekten und transparenten Verfahren zustande gekommen. ${ }^{238}$ Ausgangspunkt für jede Risikobewertung sei das Dossier, das vom jeweiligen Unternehmen zusammengestellt werde. Es sei „natürlich und notwendig, dass Teile des von dem Unternehmen übermittelten Dossiers in Abschnitten des vorläufigen Bewertungsbericht[s] erscheinen, der vom Bericht erstattenden Mitgliedstaat erstellt wird“. Unternehmen seien verpflichtet, Studien und Literatur zusammenzufassen. „Wenn der RMS [Rapporteur Member State] mit einer bestimmten Zusammenfassung oder Bewertung übereinstimmt, kann er den Text direkt im vorläufigen Bewertungsbericht übernehmen." Immerhin enthalte der RAR zahlreiche Beispiele für Änderungen im Vergleich zum ursprünglichen Unternehmensdossier. Auch das deutsche BfR bekräftigte diese Position in seiner Entgegnung zur Plagiatsstudie. ${ }^{239}$ Es werde häufig das übliche Verfahren der Wiedergenehmigung von Pflanzenschutzmittelwirkstoffen „in seiner Komplexität nicht nachvollzogen“. Im „entscheidenden“ Kapitel habe man 90\% eigenständig formuliert. Deshalb sei der Begriff „Plagiat“ in diesem Zusammenhang „nicht zutreffend“.

\section{c) Erkenntnisse aus Rechtsstreitigkeiten in den USA: Die „Monsanto Papers“}

Im Zusammenhang mit einem Zivilrechtsstreit in den USA, den ca. 60 Betroffene und Angehörige wegen Erkrankungen am Non-Hodgkin-Lymphom im Zusammenhang mit Glyphosat gegen Monsanto führten, war es dem klägerischen Anwalt gelungen, interne Dokumente von Monsanto zu beschaffen. Dabei handelte es sich vor allem um e-Mails mit angehängten Dokumenten im Umfang von einigen zehntausend Seiten, die das US-Gericht im Sommer 2017 zur Veröffentlichung freigab. ${ }^{240}$ Der Anwalt weist insbesondere auf zwei interne Programme von Monsanto hin, zum einen das Programm „Let nothing go“ - die gezielte Diskreditierung von Wissenschaftlern, die sich kritisch zu Monsanto-Produkten äußern, zum Anderen „Freedom to operate“ die Ausschaltung aller Hindernisse, die dem Produktverkauf entgegenstehen, auch

238 EFSA, Stellungnahme der EFSA zu Vorwürfen in Bezug auf den Bewertungsbericht zur Erneuerung der Genehmigung von Glyphosat vom 22.09.2017, abrufbar auf der Homepage der EFSA unter: www.efsa.europa.eu/sites/default/files/170922_glyphosate_statem ent_de.pdf (15.06.2019).

$239 B f R$, Presseinformation 02/2019 vom 15.01.2019 („Europäische Glyphosatbewertung erfolgt qualitätsgesichert und unabhängig - Berichte der Industrie sind zwangsläufig Teil der Bewertungsberichte"), abrufbar unter: www.bfr.bund.de/de/presseinformationen.html (15.06.2019).

240 Eine Fülle dieser Dokumente sowie weiterer Informationen zu den wichtigsten laufenden Verfahren mit inzwischen über 9.000 Klägern und Klägerinnen in den USA, u.a. zum Fall DeWayne Johnson v. Monsanto, sind bei USRK (U.S. Right to Know) abrufbar unter: https://usrtk.org/monsanto-papers/ (15.06.2019). Siehe auch Homepage der Anwaltskanzlei Baum Hedlund Law unter: www.baumhedlundlaw.com (15.06.2019). 
mithilfe des Einsatzes von „Mietmäulern“ aus der Wissenschaft. ${ }^{241}$ Inzwischen wurden die Monsanto Papers auch von der internationalen Presse, insbesondere „Le Monde“, zum Teil über Monate ausgewertet. ${ }^{242}$ Danach soll Monsanto systematisch, zum Teil über eine Beratungsfirma, Wissenschaftler kontaktiert haben, damit diese positive Studien über Glyphosat veröffentlichten, und dies auch bezahlt haben. Angeführt wird z.B. der Fall einer Cheftoxikologin von Monsanto, die Teile eines wissenschaftlichen Beitrags über die Auswirkungen von Glyphosat auf Fruchtbarkeit und Embryonalentwicklung vorab einem Korrektor der Zeitschrift „Journal of Toxicology and Environmental Health "übersandt und zugleich dafür gesorgt hatte, dass bei der Publikation ihr Name gestrichen wurde, so dass nach außen nur Autoren ohne Bezug zu Monsanto erschienen. Die Presse sprach unter Bezugnahme auf eine interne Sprachpraxis bei Monsanto von einer speziellen Form des „Ghostwriting“. Weitere Erkenntnisse gewinnt eine wissenschaftliche Studie im Auftrag mehrerer Umweltverbände. ${ }^{243}$ Auch das Europäische Parlament äußerte Zweifel an der Glaubwürdigkeit von Studien, auf die EFSA und ECHA ihre Risikobewertung gestützt hatten. ${ }^{244}$ Monsanto bestritt alle Manipulationsvorwürfe mit dem Argument, die Mails seien aus dem Zusammenhang gerissen. ${ }^{245}$

\section{d) Schlussfolgerungen zur Unabhängigkeit und Wissenschaftlichkeit der Bewertung}

Die Frage nach der Unabhängigkeit der wissenschaftlichen und behördlichen Bewertung kann nicht mit pauschalen Hinweisen auf eine „beispiellose Kampagne“ und „Panikmache“, die schon „den Sieg über wissenschaftlichen Sachverstand davongetragen" habe, abgetan werden. ${ }^{246}$ Denn die hier präsentierten Details stellen gewichtige Indizien für die Annahme dar, dass die Bewertung weder frei vom Einfluss der interessierten Unternehmen war, noch wissenschaftlichen Standards entsprach. Nicht ohne Grund beabsichtigt selbst die Kommission, die Funktion des berichterstattenden

241 Interview mit Brent Wisner in: Der Spiegel Nr. 34 vom 18.08.2018, S. 65 („Gift in der Geschichte").

242 Auch zum Folgenden statt Vieler: Le Monde vom 04.10.2017 (Stephane Foucard/ Stephane Horel, «Monsanto papers», désinformation organisée autour du glyphosate), sowie vom 11./13.08.2018, (Stephane Foucard, Les «Monsanto Papers», à la base de la controverse sur le glyphosate), abrufbar unter: www.lemonde.fr (15.06.2019), ferner Süddeutsche Zeitung vom 02.04.2017 (Silvia Liebrich, Was die Monsanto-Mails verraten), abrufbar unter: www.sueddeutsche.de (15.06.2019); Zeit online vom 11.10.2017 (Annika Joeres, Hat Monsanto Wissenschaftler gekauft?), abrufbar unter: www.zeit.de (15.06.2019).

243 Burtscher-Schaden/Clausing/Robinson, Glyphosat und Krebs: Gekaufte Wissenschaft/ Glyphosate and cancer, Buying science, März 2017, abrufbar auf der Seite des BUND: www.bund.net (15.06.2019).

244 Auch zum Folgenden Erwägungsgrund J sowie Ziffer 7 der Entschließung des Europäischen Parlaments vom 24. Oktober 2017, (Fn. 92). S.o. B I.3. m.N.

245 FAZ Nr. 256 vom 03.11.2018, S. 24 („Unruhe am Monsanto Drive“) unter Bezugnahme auf den Chef der Bayer-Agrosparte Liam Condon.

246 So aber Kaus, StoffR 2016, S. 110 f., 113. 
Mitgliedstaats im Falle von Glyphosat von Deutschland auf Frankreich zu übertragen. ${ }^{247}$

Die Antragstellerin (Glyphosate Task Force) war verpflichtet, die einschlägige offen zugängliche Literatur sowie Informationen über alle durchgeführten Versuche und Studien vollständig in ihren Dossiers einzureichen. ${ }^{248}$ Dasselbe gilt für die mit der Wirkstoff-Bewertung befassten europäischen und deutschen Behörden. Auch sie sind nach allen geltenden Grundsätzen und Leitlinien verpflichtet, relevante Studien vollständig heranzuziehen und nach wissenschaftlichen Methoden unter Zuhilfenahme von Fachleuten objektiv und transparent zu bewerten. Das ist nicht geschehen. Denn, wie gezeigt, hat EFSA das Fehlen von 7 Studien mit Tumorbefunden im Zusammenhang mit Glyphosat in Bewertungsberichten des BfR und des eigenen Riskobewertungsausschusses RAC damit zu erklären versucht, dass man sie nicht übersehen oder weggelassen, sondern nur als „nicht gefahren- und bewertungsrelevant“ eingeschätzt habe. Das hätte sie dann allerdings offenlegen und begründen müssen, wie es die Regeln über die Datenanforderungen ausdrücklich vorgeben. ${ }^{249}$ Unsichtbare „Vorabbewertungen" entsprechen auch nicht den Regeln guter wissenschaftlicher Praxis. Denn wenn eine Studie thematisch einschlägig ist, muss sie zitiert und transparent bewertet werden, damit auch gerade die Bewertung als „nicht relevant“ dem kritischen externen Urteil zugänglich wird.

Soweit es die Plagiatsvorwürfe betrifft, rechtfertigen sich EFSA und BfR damit, dass es ganz normal sei, eine Zusammenfassung oder Bewertung direkt in den Bewertungsbericht zu übernehmen, wenn man mit dem Text übereinstimme. Das ist allerdings nur dann der Fall, wenn die Originalquelle zitiert wird. Dagegen begründet es schwerwiegendes wissenschaftliches Feblverhalten, wenn diese Offenlegung unterbleibt, ${ }^{250}$ so wie es die Plagiatsstudie von 2019 für viele und auch relevante (bewertende) Stellen nachweist. Zu diesem Vorwurf fehlender Quellenangaben nehmen die beschuldigten Behörden keine Stellung, so dass er auch nicht als entkräftet angesehen werden kann. Nicht entscheidend ist, dass das finale Kapitel, wie vom BfR vorgetragen, $\mathrm{zu}, 90 \%$ “ eigenständig formuliert sei. Denn die eigenständige Formulierung eines Schlusskapitels heilt nicht Fehler und Regelverletzungen, die im dafür grundlegenden Hauptteil begangen wurden. Warum selbst hier noch 10\% ohne Kenntlichmachung kopiert wurden, erklären die beschuldigten Behörden ebenfalls nicht.

Im Falle der „Monsanto Papers“ geben die in den USA gefundenen Unterlagen Anlass zu der Annahme, dass wissenschaftliche Studien durch Monsanto finanziert und teilweise auch verfasst wurden, ohne dass dies nach außen transparent gemacht

247 Marks, Brussels moves goalposts on glyphosate: Commission plans to shift responsibility from Germany to France for evaluating controversial weedkiller, Politico 18.07.2018, abrufbar unter: www.politico.eu (15.06.2019), unter Berufung auf einen Entwurf der Kommission (SANTE/10421/2018 Rev. 1) zur Änderung der Verordnung (EU) 686/2012.

248 Nr. 1.5 des Anhangs zur VO (EU) 283/2013 (Datenanforderungen für die Wirkstoffgenehmigung); Nr. 1.5 VO (EU) 284/2013 (Datenanforderungen für Pflanzenschutzmittelzulassung).

249 Nrn. 1.5 des Anhangs zur VO (EU) 283/2013 bzw. 284/2013.

250 S.o. C I.2.c) m.N. 
wurde. Dies stellt eine besonders schwere Form des wissenschaftlichen Fehlverhaltens dar. ${ }^{251}$

Die Ergebnisse dieses Abschnitts können dahingehend zusammengefasst werden, dass schwerwiegende Zweifel an einer unabhängigen Bewertung durch BfR und EFSA bestehen. Dabei geht es nicht um „Missverständnisse in der öffentlichen Wahrnehmung“, die durch eine erhöhte Transparenz der Berichterstattung und „Optimierung der Darstellung " ausgeräumt werden könnten. ${ }^{252}$ Betroffen ist hier vielmehr die sachliche Qualität der Bewertung, an der begründete Zweifel bestehen. Diese Zweifel können auch nicht durch Hinweise auf eine Liste von vielen anderen internationalen und staatlichen Institutionen zerstreut werden, die ebenfalls keine Gesundheitsgefahren in Bezug auf Glyphosat erkennen. ${ }^{253}$ Denn erstens kann aus dem bloßen Umstand, die Mehrheit zu stellen oder am stärksten verbreitet zu sein, nicht auf die Unabhängigkeit oder Richtigkeit des Urteils geschlossen werden. Zweitens wissen wir nicht, wie unabhängig diese Institutionen ihrerseits sind.

\section{Pflanzenschutzmittelzulassung durch die Mitgliedstaaten, insbesondere Deutschland}

\section{Europäische Vorgaben}

\section{a) Grundlegende Anforderungen und „zonales Verfabren“}

Die EU-Mitgliedstaaten sind zwar zuständig für die Zulassung von Pflanzenschutzmitteln. ${ }^{254}$ Dabei unterliegen sie jedoch einer ganzen Reihe von Vorgaben aus den Art. 28-54 der Pflanzenschutzmittelverordnung (EG) 1107/2009.255 Anders als beim normalen Warenverkehr innerhalb der EU genügt es nicht, dass ein Pflanzenschutzmittel in einem Mitgliedstaat zugelassen ist, um es auch in alle anderen zu exportieren (Prinzip der gegenseitigen Anerkennung). Vielmehr muss entweder eine Zulassung in jedem einzelnen Mitgliedstaat beantragt werden, in dem das Mittel in Verkehr gebracht werden soll (Art. 33 Abs. 1 VO [EG] 1107/2009), oder es muss ein Verfahren der gegenseitigen Anerkennung (Art. 40 ff. VO [EG] 1107/2009) durchlaufen werden. Besondere Regeln gelten für Parallelexporte (Art. 52 VO [EG] 1107/2009).

Materiell-rechtlich entscheidend ist, ob ein Pflanzenschutzmittel die Voraussetzungen des Art. 29 VO [EG] 1107/2009 für die „Zulassung zum Inverkehrbringen“ erfüllt. Von den zahlreichen Anforderungen sollen hier nur folgende herausgehoben werden: Wichtigste Voraussetzung ist, dass die im Mittel enthaltenen Wirkstoffe sowie „Safener" und „Synergisten“ auf europäischer Ebene ${ }^{256}$ zugelassen sind (Art. 29 Abs. 1 lit. a VO [EG] 1107/2009). Ferner muss das Pflanzenschutzmittel „unter Be-

251 S.o. C I.2.c) m.N.

252 BfR 2019, (Fn. 239).

253 Ibid.

254 Art. 33 Abs. 1, Art. 36 Abs. 2 VO (EG) 1107/2009.

255 S.o. B II.

256 Kapitel II der VO (EG) 1107/2009. S.o. B I und C I. 
rücksichtigung des nenesten Stands von Wissenschaft und Technik" die Anforderungen des Art. 4 Abs. 3 VO (EG) 1107/2009 erfüllen (Art. 29 Abs. 1 lit. e VO [EG] 1107/2009). Danach müssen insbesondere „schädliche Auswirkungen“ auf die Gesundheit von Mensch und Tier (Art. 4 Abs. 3 lit. b) sowie „unannebmbare Auswirkungen" auf die Umwelt, insbesondere die biologische Vielfalt und das Ökosystem (Art. 4 Abs. 3 lit. e), ausgeschlossen sein. An dieser Stelle verweist das Kapitel zur Pflanzenschutzmittelzulassung auf die pflanzenschutzmittelspezifischen Bestimmungen $^{257}$ im Kapitel zur Wirkstoffzulassung.

Das Verfahren für die Bewertung und Zulassung von Pflanzenschutzmitteln ist in Art. 33 ff. VO (EG) 1107/2009 geregelt. Wie gezeigt, bedarf es zwar der Zulassung in jedem einzelnen Mitgliedstaat, für die Bewertung gilt aber ein zonales Verfahren. Das heißt, nur ein Mitgliedstaat nimmt diese mit Wirkung für alle anderen Mitgliedstaaten vor, die sich in derselben geografischen Zone befinden (Art. 35 f. VO [EG] 1107/2009). Aus Anlage I zur VO (EG) 1107/2009 ergibt sich, dass Deutschland zusammen mit Österreich, den Benelux-Staaten, den osteuropäischen Staaten (ohne Baltikum und Bulgarien), den Balkanstaaten sowie Irland und dem Vereinigten Königreich zur „Zone B - Mitte" gehört. An dieser entscheidenden Stelle gilt also doch das Prinzip des gegenseitigen Vertrauens der Mitgliedstaaten in einem begrenzten Rahmen. Indem die aufwändige Bewertung zonal konzentriert wird, werden der Binnenmarkt gestärkt, überflüssige Mehrfachstudien (insbesondere an Tieren) vermieden, - aber auch das Verfahren verzögert, weil auf Ergebnisse aus dem europäischen Ausland gewartet werden muss. Für die Art und Weise der Bewertung gelten dieselben Bewertungsmaßstäbe wie bei der Wirkstoffbewertung, insbesondere die „einheitlichen Grundsätze" für die Bewertung und Zulassung von Pflanzenschutzmitteln aus Art. 29 Abs. 6 VO (EG) 1107/2009.258 Ein Problem liegt allerdings darin, dass jede Antragstellerin grundsätzlich wählen kann, welcher Mitgliedstaat ihren Antrag prüfen soll, und damit auch jenen Staat wählt, der über die Vertraulichkeit von Unternehmensdaten entscheidet, ${ }^{259}$ die der Bewertung zugrunde liegen. Das eröffnet nicht nur Möglichkeiten für Unternehmen, das Land zu wählen, das möglichst großzügig in ihrem Sinne verfährt, sondern auch, die Beziehungspflege zu den staatlichen Zulassungsbehörden auf einige wenige Länder zu konzentrieren und damit effizienter zu betreiben.

\section{b) Verweigerung der Zulassung und Zulassung unter Auflagen}

Von großer Bedeutung ist die Frage, wieviel Gestaltungsspielraum den Mitgliedstaaten bei der Zulassung konkreter Anwendungsprodukte noch verbleibt, nachdem der in ihnen enthaltene Wirkstoff (z.B. Glyphosat) genehmigt worden ist. Kann z.B. ein einzelner Mitgliedstaat, der dem Wirkstoff kritisch gegenübersteht, so restriktiv mit der Zulassung der Anwendungsprodukte verfahren, dass zumindest in diesem Land letztlich auch der Wirkstoff faktisch von Feldern, Weinbergen und Obstplantagen

257 S.o. C I.2.b).

258 S.o. C I.2.c).

259 Art. 35 und Art. 33 Abs. 4 VO (EG) 1107/2009. 
verschwindet? Aus der Durchführungsverordnung (EU) 546/2011 zu den einheitlichen Grundsätzen für die Bewertung und Zulassung von Pflanzenschutzmitteln gemäß Art. 29 Abs. 6 VO (EG) 1107/2009 ergibt sich nur, dass die Mitgliedstaaten die der Kommission zum Zweck der Zulassung des Wirkstoffs vorgelegten Angaben zu dem Wirkstoff des Pflanzenschutzmittels sowie die Ergebnisse ihrer Bewertung „berücksichtigen". ${ }^{260}$ Das wird zum Teil als Gewährung von Freiraum bzw. fehlende „Präjudizwirkung“ der Wirkstoffgenehmigung gedeutet. ${ }^{261}$ Auch nach Auffassung des EuG „verfügen die Mitgliedstaaten im Rabmen der Prüfung der in Art. 29 der Verordnung Nr. 1107/2009 genannten Anforderungen für die Zulassung über ein beträchtliches Ermessen und einen erheblichen Handlungsspielraum ". ${ }^{262}$ Die betreffenden Entscheidungen stehen allerdings alle im Kontext von Individualklagen gegen die Wirkstoffgenehmigung, die allesamt als unzulässig abgewiesen wurden, weil nach Auffassung des Gerichts erst die Zulassung der Anwendungsmittel zu einer hinreichend individualisierten Betroffenheit (Art. 263 Abs. 4 AEUV) der Klagenden führt. Diese Rechtsprechung beruht auf der Prämisse, dass aus der Wirkstoffgenehmigung nicht automatisch die Zulassung von Pflanzenschutzmitteln folgt, sondern die Mitgliedstaaten durchaus eigene Entscheidungen treffen.

Wie groß der Handlungsspielraum der Mitgliedstaaten tatsächlich ist, muss letztlich anhand der Zielrichtung und Systematik der zweistufigen bzw. zonalen Bewertung beurteilt werden. Sinn macht diese nämlich nur dann, wenn die Wirkstoffgenehmigung nicht ohne Weiteres durch jeden Mitgliedstaat im Kontext der folgenden $\mathrm{Zu}$ lassungsentscheidung neutralisiert werden kann. Dasselbe gilt in Bezug auf die zonale Bewertung. Deshalb warten die Mitgliedstaaten einer Zone, für die eine Zulassung für ein Produkt beantragt wurde, die Bewertung durch den „den Antrag prüfenden Mitgliedstaat" $a b$ und gewähren oder verweigern die Zulassung dann „auf der Grundlage der Schlussfolgerungen aus der Bewertung" durch den Prüfstaat (Art. 35, 36 Abs. 2 VO [EG] 1107/2009). Entsprechendes gilt für die gegenseitige Anerkennung eines Pflanzenschutzmittels, das innerhalb derselben Zone durch einen anderen Mitgliedstaat zugelassen wurde (Art. 41 Abs. 1 VO [EG] 1107/2009). ${ }^{263}$ Das bedeutet, dass die

260 Anhang zur Durchführungsverordnung (EU) 546/2011, (Fn. 138), Teil I, Abschnitt A, Nr. 2b.

261 S. etwa Doubaire, ZUR 2017, S. 397 f.

262 EuG, T-600/15, Pesticide Action Network (PAN) u.a./Kommission, ECLI:EU:T:2016:601, Rn. 33. S.a.E III.

263 Siehe VG Braunschweig, VG Braunschweig, Urt. v. 30.11.2016, Az. 9 A 28/16, BeckRS 2016, 110721: „Zur Vermeidung von Doppelarbeit, Verringerung des Verwaltungsaufwands für Industrie und Mitgliedstaaten und zur Sicherstellung einer einheitlicheren Verfügbarkeit von Pflanzenschutzmitteln soll die von einem Mitgliedstaat erteilte Zulassung von anderen Mitgliedstaaten akzeptiert werden, sofern die landwirtschaftlichen, pflanzengesundheitlichen und ökologischen Bedingungen (einschließlich der klimatischen Bedingungen) vergleichbar sind. [...] Anhaltspunkte für eine in diesem Sinne systematische Verletzung von Rechtsvorschriften des Zulassungsverfahrens durch den Referenzmitgliedstaat Großbritannien sind nicht erkennbar und von der Beklagten auch nicht vorgetragen worden. Nicht jede Verletzung von Rechtsvorschriften des Zulassungsverfahrens kann zur Widerlegung der auf dem Prinzip gegenseitigen Vertrauens berubenden Vermutung, die vom Referenzmitgliedstaat erteilte Zulassung entspreche den europarechtlichen Vorgaben, als genügend angesehen werden". 
Mitgliedstaaten grundsätzlich keine erneute Bewertung durchführen dürfen. Wollen sie die Zulassung trotz einer positiven Bewertung erschweren bzw. verweigern, bleiben ihnen nur die Möglichkeiten des Art. 36 Abs. 3 VO (EG) 1107/2009. Danach müssen zuerst Risikominderungsmaßnahmen ausgeschöpft werden, die aber nur zulässig sind, soweit es um die „spezifischen Verwendungsbedingungen“ eines bestimmten Pflanzenschutzmittels geht. Allerdings wird zum Teil für eine weite Auslegung dieses Begriffes plädiert, wonach risikomindernde Einschränkungen auch zulässig wären, wenn anderenfalls das „hohe Schutzniveau“ für Gesundheit und Umwelt nicht erreicht oder der nationale Aktionsplan nicht verwirklicht werden kann. ${ }^{264}$ Nur wenn die Bedenken eines Mitgliedstaats in Bezug auf die Gesundheit von Mensch und Tier oder die Umwelt dadurch nicht ausgeräumt werden können, kann er die Zulassung in seinem Gebiet ganz verweigern. Dies setzt allerdings voraus, dass er „angesichts spezifischer ökologischer oder landwirtschaftlicher Bedingungen berechtigten Grund zu $\operatorname{der}$ Annabme hat, dass das betreffende Produkt noch immer ein unannehmbares Risiko für die Gesundheit von Mensch und Tier oder die Umwelt darstellt“. ${ }^{265}$ Die Generaldirektion Gesundheit und Lebensmittelsicherheit der Kommission hat in einem „Leitlinien-Dokument“ zur Pflanzenschutzmittelzulassung klargestellt, dass dies nicht zur erneuten Bewertung des Zulassungsantrags berechtige. Vielmehr sollen die Mitgliedstaaten ihre Bewertung auf die besonderen nationalen Bedürfnisse beschränken. ${ }^{266} \mathrm{Die}$ Mitgliedstaaten haben also nicht ohne Weiteres die Möglichkeit, eine eigenständige „Ausstiegsstrategie“ zu verwirklichen.

Ähnliches gilt in Bezug auf Art. 31 VO (EG) 1107/2009, wonach die Mitgliedstaaten Pflanzenschutzmittel unter Auflagen zulassen (Absätze 2 und 3) und den Zweck ihrer Verwendung bestimmen (Absatz 1) können. Durch Auflagen soll vor allem sichergestellt werden, dass Anforderungen aus der Wirkstoff-Genehmigungsverordnung, die auf den Bereich der Anwendung zielen, tatsächlich bei der Zulassung der einzelnen Anwendungsprodukte umgesetzt werden. Auch dieses Mittel ermöglicht aber keine Neubewertung von Aspekten, die schon Gegenstand der Wirkstoffgenehmigung oder der zonalen Bewertung zum Zwecke der Zulassung von Pflanzenschutzmitteln gewesen waren. Auflagen nach Art. 31 VO (EG) 1107/2009 müssen deshalb auf besondere Auswirkungen des jeweiligen Pflanzenschutzmittels vor Ort ${ }^{267}$ oder veränderte Verhältnisse bezogen sein.

264 Doubaire, ZUR 2017, S. 398 f. A.A. Koof, StoffR 2016, S. 120, 123: nur landesspezifische Eigenheiten zählen, da das zonale System ein antizipiertes Sachverständigengutachten mit Einbindung alle Mitgliedstaaten der jeweiligen Zone beinhalte.

265 Art. 36 Abs. 3 sowie Erwägungsgrund 29 der VO (EG) 1107/2009.

266 Kommission, Guidance document on zonal evaluation and mutual recognition under Regulation (EC) No. 1107/2009, SANCO/13169/2010 rev. 9, S. 14 und 16, abrufbar unter: https://ec.europa.eu/food/sites/food/files/plant/docs/pesticides_ppp_appproc_guide_m ut-rec_en.pdf (15.06.2019).

267 Gutachten des Wissenschaftlichen Dienstes des Deutschen Bundestages, Die Möglichkeit nationaler Verbote von Glyphosat, Die Rechtslage nach der erneuten Zulassung auf Unionsebene, Az. PE 6 - 3000 - 95/17, 12.12.2017, S. 8. 


\section{c) Erneuerung der Zulassung}

Für die Erneuerung der Zulassung eines Pflanzenschutzmittels gelten besondere Regeln. Antragsteller müssen innerhalb von drei Monaten nach der Erneuerung der Genehmigung eines Wirkstoffs einen Antrag auf Neuzulassung von Pflanzenschutzmitteln mit diesem Wirkstoff stellen, die Mitliedstaaten spätestens zwölf Monate nach der erneuten Wirkstoffgenehmigung ihre Entscheidung über die Neuzulassung der Pflanzenschutzmittel getroffen haben (Art. 43 Abs. 2 und 5 VO [EG] 1107/2009). Grundsätzlich wird die Laufzeit der Zulassung für ein Pflanzenschutzmittel auf nicht länger als ein Jahr nach dem Auslaufen der Wirkstoffgenehmigung festgelegt, um eine Neuzulassung des Mittels im direkten Anschluss an die alte zu ermöglichen, und danach (nur) solange, wie die Wirkstoffgenehmigung läuft (Art. 32 VO [EG] 1107/2009). Das Schicksal der Zulassung eines Anwendungsmittels ist also an dasjenige des Wirkstoffs geknüpft. Nur so kann sichergestellt werden, dass neue Bedingungen und Verschärfungen in einer erneuerten Wirkstoffgenehmigung sich auch bei allen Anwendungsprodukten realisieren lassen (vgl. Art. 43 abs. 3 VO [EG] 1107/2009). Scheitert der Wirkstoff, fällt auch das Pestizid, das ihn enthält, der „Diskontinuität“ anheim.

Grundsätzlich „wird“ eine auslaufende Zulassung erneuert, wenn die Voraussetzungen des Art. 29 VO (EG) 1107/2009 noch vorliegen (Art. 43 Abs. 1 VO [EG] 1107/2009). ${ }^{268}$ Die Formulierung kann dabei nur so verstanden werden, dass im Falle der Erfüllung der Anforderungen auch ein Rechtsanspruch auf Neuzulassung besteht, der vor mitgliedstaatlichen Gerichten eingeklagt werden kann. ${ }^{269}$ Wurde ein Wirkstoff - wie hier Glyphosat - auf der europäischen Ebene neu genehmigt, müssen die Mitgliedstaaten auch prüfen, ob „Altmittel“, für die in diesem Falle stets eine Neuzulassung zu beantragen ist (!), die neuen Bedingungen und Einschränkungen der Verordnung zur Erneuerung der Genehmigung des Wirkstoffs erfüllen (Art. 43 Abs. 3 VO [EG] 1107/2009). In diesem Erfordernis der „rechtlichen Aktualisierung“ zeigt sich einmal mehr die grundlegende Abhängigkeit der Pflanzenschutzmittelzulassung von der Wirkstoffgenehmigung. ${ }^{270}$ Soweit die Prüfungsbefugnis im Rahmen der faktischen und rechtlichen Aktualisierung gemäß Art. 43 reicht, findet auch im Erneuerungsverfahren eine zonale Bewertung statt (Art. 43 Abs. 3 UAbs. 2 VO [EG] 1107/2009).

Schwierig zu beurteilen ist, ob auch im Erneuerungsverfahren eine Zulassungsverweigerung auf der Basis des Art. 36 Abs. 3 VO (EG) 1107/2009 (spezifische ökologische oder landwirtschaftliche Bedingungen im jeweiligen Land) in Betracht kommt, da Art. 43 VO (EG) 1107/2009 nicht auf diese Vorschrift verweist. Andererseits verweist Art. 43 auch nicht explizit auf Art. 31, was aber unabdingbar ist, um z.B. verschärfte Anforderungen aus einer erneuerten Wirkstoffgenehmigung durch Auflagen bei der Neuzulassung von Pflanzenschutzmitteln durchzusetzen. Art. 43 kann des-

268 S.o. C II.1.a).

269 Art. 36 Abs. 3 UAbs. 4 VO (EG) 1107/2009 dürfte, obwohl Art. 43 VO (EG) 1107/2009 nicht direkt auf ihn verweist, auch für die Verweigerung der Erneuerung einer Zulassung gelten.

270 Vgl. Art. 43 Abs. 3 VO (EG) 1107/2009. 
halb nicht so verstanden werden, als würde er nur zwei Arten von Anforderungen tolerieren, die in ihm selbst genannt sind, nämlich diejenigen des Art. 29 und diejenigen der Verordnung zur Neugenehmigung des Wirkstoffs. Für eine Geltung von Art. 36 Abs. 3 auch bei der Erneuerung einer Zulassung spricht, dass sich „spezifische ökologische und landwirtschaftliche Bedingungen“ ändern können und deshalb auch die Spezifität einer Aktualisierung bedarf. Allerdings muss beachtet werden, dass Art. 36 Abs. 3 eine Ausnahmeregel ist, die die Ergebnisse der Wirkstoff- und der zonalen Bewertung durchkreuzen kann. Das könnte dafür sprechen, sie im Erneuerungsverfahren nur heranzuziehen, wenn sich Änderungen der Vor-Ort-Situation ergeben haben. Eine verbindliche Klärung der Frage steht aber noch aus.

\section{Deutsche Regelungen zur Pflanzenschutzmittel-Zulassung}

Die Regelungen der europäischen Pflanzenschutzmittelverordnung (EG) 1107/2009 sind grundsätzlich unmittelbar anwendbar in Deutschland (Art. 288 UAbs. 2 AEUV), werden jedoch durch das Pflanzenschutzgesetz (PflSchG), ${ }^{271}$ die Pflanzenschutzmittelverordnung (PflSchMV)272 und die Pflanzenschutz-Anwendungsverordnung (PflSchAnwV) $)^{273}$ weiter konkretisiert.

Soweit es Entscheidungen über die Zulassung von Pflanzenschutzmitteln betrifft, entscheidet letztlich das BVL, allerdings in einem hochkomplexen Verfahren mit zahlreichen Akteuren. Wie gezeigt, gilt auch bei der Zulassung von Pflanzenschutzmitteln das zonale Verfahren (Art. 35 f. VO [EG] 1107/2009). Ist Deutschland bewertender Mitgliedstaat, erarbeitet das BVL einen vorläufigen Zulassungsbericht, an dessen Entstehung das BfR, das Julius-Kühn-Institut und das Umweltbundesamt (UBA) im Rahmen ihrer jeweiligen Zuständigkeiten mit eigenen Bewertungsberichten mitwirken. Das Umweltbundesamt muss mit Blick auf seine Kompetenz zur Bewertung von Umweltauswirkungen sein Einvernehmen erteilen. Danach erhalten die anderen EU-Mitgliedstaaten derselben Zone Gelegenheit zur Stellungnahme zum vorläufigen Bewertungsbericht (Art. 36 Abs. 1 UAbs. 3 VO [EG] 1107/2009). Nach Überarbeitung des Berichts durch die deutschen Behörden trifft das BVL dann die Entscheidung, ob und mit welchen Auflagen die Zulassung erneuert wird. ${ }^{274}$ Wie noch mit Bezug auf die glyphosathaltigen Anwendungsmittel gezeigt werden wird, führt der hiermit verbundene Zeitaufwand zu einer Situation, in der die Verwaltung ihre Aufgaben nicht mehr fristgemäß erfüllen kann.

Die Gewährleistung der Sicherheit der Pestizidzulassung durch das BVL verläuft auf zwei Schienen. Zum einen bestimmt $\$ 35$ Abs. 1 PflSchG, dass bei der Prüfung eines Antrags auf Zulassung eines Pflanzenschutzmittels, dessen Wirkstoff nach

271 S.o. Fn. 16.

272 Verordnung über Zulassungs- und Genehmigungsverfahren für Pflanzenschutzmittel vom 15. Januar 2013, BGBl. I S. 74.

273 Verordnung über Anwendungsverbote für Pflanzenschutzmittel vom 10. November 1992 (BGBl. I S. 1887), zuletzt geändert durch Artikel 1 der Verordnung vom 25. November 2013 (BGBl. I S. 4020).

274 S. i.E. $\int \mathbb{S} 28 \mathrm{ff}$. PflSchG. Zu den Zuständigkeiten bereits o. B II.1. 
Art. 4 VO (EG) 1107/2009 genehmigt wurde, „die aus dem Genehmigungsverfahren abgeleiteten Erkenntnisse über die Eigenschaften des Wirkstoffes zu Grunde zu legen" und die Leitlinien der Kommission für die Antragstellung (Art. 77 VO [EG] 1107/2009) zu beachten sind. Damit soll die Beachtung der unionsrechtlichen Entscheidung über den Wirkstoff, einschließlich ihrer Bewertungsgrundlagen, sichergestellt werden. Zum anderen kann das BVL gemäß \$36 PflSchG in der Zulassung ergänzend $\mathrm{zu}$ den in Art.31 Abs.2, 3 VO (EG) 1107/2009 vorgeschriebenen Bestimmungen (Auflagen aus der DurchführungsVO zur Wiedergenehmigung des Wirkstoffs, Einstufungsanforderungen betreffend Etikettierung, Höchstdosis u.s.w.) weitere Anwendungsbestimmungen zum Schutz der Gesundheit von Mensch und Tier und zum Schutz vor sonstigen schädlichen Auswirkungen festlegen. Unproblematisch ist dies insoweit, als es um spezifische Eigenschaften bestimmter Pflanzenschutzmittel unter bestimmten landestypischen Gegebenheiten geht, d.h. um Gefahren, die noch nicht Gegenstand vorangegangener Bewertungen auf der Unionsebene oder im Rahmen einer zonalen Bewertung gewesen waren, oder um risikomindernde Maßnahmen, die speziell die Verwendungsbedingungen betreffen (Art. 36 Abs. 3 UAbs. 1 VO (EG) 1107/2009). Darüber hinaus kommen ergänzende Maßnahmen bei Änderungen der Situation einschließlich neuer wissenschaftlicher Erkenntnisse in Betracht. ${ }^{275}$ Diese Anforderungen müssen auch die Landesbehörden bei der Wahrnehmung der Überwachungsaufgaben vor Ort beachten, ${ }^{276}$ ohne dass die Bundesbehörden ihnen aber konkrete Anweisungen erteilen dürfen (Art. 83 GG). Da die Bedeutung des landwirtschaftlichen Sektors und die politische Interessenlage der einzelnen Bundesländer unterschiedlich ausfallen, kann auch die Intensität von Überwachungsmaßnahmen von Bundesland zu Bundesland schwanken. Den offenbar erwarteten Vollzugsdefiziten in diesem Bereich versucht man mit einem gemeinsamen Bund-Länder„Pflanzenschutz-Kontrollprogramm“ beizukommen. ${ }^{277}$

Die Frage, inwieweit die Nutzung glyphosathaltiger Pflanzenschutzmittel in Deutschland eingeschränkt ist, beantwortet sich in erster Linie nach der Pflanzenschutz-Anwendungsverordnung (PflSchAnwV), deren letzte Änderung allerdings vom November 2013 datiert. ${ }^{278}$ Die Bundesregierung beabsichtigt, sowohl die eigene Glyphosat-Reduktionsstrategie als auch die unionsrechtlichen Vorgaben, insbesondere diejenigen aus der DurchführungsVO zur erneuten Genehmigung von Glyphosat, durch Änderung der Pflanzenschutz-Anwendungsverordnung umzusetzen. Die betreffende Änderungsverordnung wird auf die Verordnungsermächtigung des \$14 PflSchG gestützt werden. Ende August 2018 befanden sich aber praktisch alle wesentlichen Fragen zur Reduktionsstrategie noch in den Ressortberatungen. ${ }^{279}$

Die Pflanzenschutz-Anwendungsverordnung unterscheidet zwischen vollständigen und eingeschränkten Anwendungsverboten ( $\mathbb{S} 1 \mathrm{f}$.) sowie Anwendungsbeschränkungen ( $(3)$, denen jeweils eine Anlage korrespondiert. Für Glyphosat und Glypho-

275 Zur Frage, wieviel Handlungsspielraum den Mitgliedstaaten verbleibt, o. C II.3.c).

276 Zur Zuständigkeit der Landesbehörden oben B II.2.

277 S.u. D II.3.

278 S.o. Fn. 273.

279 BReg, BT-Drs. 19/4097 vom 28.08.2018, (Fn. 24), S. 5 (zu Fragen 8-11), 7 (zu Frage 23). 
sat-Trimesium bestehen bislang (Stand: April 2019) lediglich Anwendungsbeschränkungen. Konkret ist nur die Anwendung auf versiegelten und befestigten Flächen verboten, von denen die Gefahr einer Abschwemmung in Gewässer oder Kanäle besteht (Anlage 3 PflSchAnwV). Zudem gelten spezielle Abgabebedingungen $(\mathbb{3}$ a i.V.m. Anlage $4 \mathrm{PflSchAnwV})$ und ist der Einsatz in Naturschutzgebieten und Naturparks grundsätzlich untersagt ( $\$ 4$ i.V.m. Anlage 3 PflSchAnwV). Anders als bei anderen Wirkstoffen in der Liste ist aber weder die Nutzung im Haus- und Kleingartenbereich noch das Versprühen mit Flugzeugen generell verboten. Es gelten insoweit nur die allgemeinen Anforderungen aus $\mathbb{S} 13 \mathrm{ff}$. PflSchG. Danach müssen Pflanzenschutzmittel eigens für nichtberufliche Anwender und Anwenderinnen zugelassen sein und dürfen generell nicht verwendet werden, wenn mit schädlichen Auswirkungen auf die Gesundheit von Mensch und Tier oder den Naturhaushalt zu rechnen ist. Auf öffentlich zugänglichen Flächen wie Parks, Sport- oder Spielplätzen dürfen nicht gänzlich unbedenkliche Pflanzenschutzmittel nur ausgebracht werden, wenn ihre Eignung für diese Flächen in einem speziellen Zulassungsverfahren festgestellt ist ( $\mathbb{1 7} \mathrm{PflSchG).} \mathrm{Schließlich} \mathrm{ist} \mathrm{das} \mathrm{Versprühen}$ durch Luftfahrzeuge generell stark eingeschränkt, Genehmigungen können aber z.B. für den Weinbau in Steillagen erteilt werden ( $\$ 18 \mathrm{PflSchG).} \mathrm{Insgesamt} \mathrm{betrachtet} \mathrm{gibt}$ das deutsche (Verordnungs-)Recht bislang weder die Beschränkungen wieder, die die europäische Durchführungsverordnung zur erneuten Genehmigung von Glyphosat vor weit über einem Jahr eingeführt hatte, noch hat die Bundesregierung ihre eigenen Ankündigungen zur Einschränkung des Gebrauchs umgesetzt.

Während man praktisch keine konkreten Ansätze zur Einschränkung des Glyphosatgebrauchs in Deutschland findet, wird in der Literatur schon seit Längerem vorgeschlagen, zur Stärkung der Biodiversität flächenbezogene Anwendungsbestimmungen wie z.B. die Ausweisung ökologischer Vorrangflächen im Rahmen des Zulassungsverfahrens festzuschreiben. ${ }^{280}$ Wer Pestizide benutzt, soll auch Blüh- und Brachflächen vorhalten müssen. Dieser Auffassung schließt sich inzwischen auch das Umweltbundesamt an, ohne sich damit jedoch durchsetzen zu können. ${ }^{281}$ Als Rechtsgrundlage hierfür werden $\$ 36$ Abs. 1 PflSchG und Art. 31 Abs. 2, 4a bzw. Art. 36 Abs. 3 UAbs. 1 VO (EG) 1107/2009 genannt. Zugleich werden allerdings Bedenken hinsichtlich der Verhältnismäßigkeit gehegt, weshalb in Anlehnung an vorhandene Greening-Modelle eine Mindestgröße von Ackerflächen angesetzt und Auswahlmöglichkeiten hinsichtlich der konkreten Maßnahmen gelassen werden sollten. Der darin ausgedrückte Ansatz, dass die flächenbesitzenden Betriebe nicht zu stark für die Biodiversität in Anspruch genommen werden dürften, überzeugt allerdings nicht. Denn die Durchführungsverordnung (EU) 2017/2324 zur erneuten Genehmigung von Glyphosat verpflichtet die Mitgliedstaaten ausdrücklich, auf die „Bedrohung der Vielfalt und Abundanz von Nichtziel-Landarthropoden und -Landwirbeltieren durch trophische Wechselwirkungen“ zu achten. Die Schutzmaßnahmen zur Rettung der biologischen Vielfalt stellen sich somit als grundlegende Bedingung für die erneute Ge-

280 Borwieck, ZUR 2017, S. 392 f.

281 S.u. C II.3.a). 
nehmigung des Wirkstoffs Glyphosat i.S.v. Art. 31 Abs. 2 VO (EG) 1107/2009 dar. Anders ausgedrückt: Ohne sie hätte es keine erneute Wirkstoffgenehmigung gegeben und dürfte nach Ablauf der Aufbrauchfrist (Art. 46 und 5 VO [EG] 1107/2009) für Altmittel niemand mehr glyphosathaltige Pestizide benutzen. Folglich müssen Auflagen zum Schutz der Umwelt im erforderlichen Umfang grundsätzlich hingenommen werden, und zwar von allen Betrieben, die auf diese Mittel nicht verzichten wollen. Der Status des „Nichtstörers“, der gewissermaßen unbeteiligt ein Opfer für den Schutz eines Allgemeinguts (Umwelt) erbringen müsste, kommt ihnen nicht zu.

\section{Neuzulassung glyphosathaltiger Mittel}

\section{a) Neuzulassungsanträge in Deutschland}

Wendet man Art. 43 Abs. 2 und 5 VO (EG) 1107/2009 auf den Glyphosatfall an, müsste für alle glyphosathaltigen Mittel innerhalb von drei Monaten nach Erneuerung der Wirkstoffgenehmigung, d.h. bis zum 15. März 2018, die Neuzulassung beantragt und vom zuständigen Mitgliedstaat innerhalb eines Jahres nach Erneuerung der Wirkstoffgenehmigung, d.h. bis 15. Dezember 2018, darüber entschieden worden sein. Denn die neue Genehmigung für den Wirkstoff Glyphosat läuft vom 16. Dezember 2017 (Datum der Genehmigung) an bis zum 15. Dezember 2022. ${ }^{282}$

Nach der aktuellen Liste des zuständigen BVL ( $\$ 33$ PflSchG) besitzen 107 glyphosathaltige Mittel in Deutschland eine Zulassung. Darauf befinden sich glyphosathaltige Mittel für unterschiedliche Einsatzbereiche mit Bezeichnungen wie "clinic“, „Bayer Garten Unkrautfrei“, „Glyfos“ (4 Varianten), „Permaclean“, „MON“ (7 Varianten), „Roundup“ (16 Varianten), etc. ${ }^{283}$ Die meisten glyphosathaltigen Mittel sind bis in den Dezember 2019 zugelassen, einige aber auch deutlich länger, so z.B. „Roundup REKORD“ und „MON 9991-SG “ bis Ende 2024 (letzte Zulassung: 25.3.2014 bzw. 3.4.2014), „Excel DF Gold“ und „Vorox Unkrautfrei direkt“ aber sogar bis Ende 2026 (letzte Zulassung: 2.5.2016 bzw. 1.2.2017).

Im Zusammenhang damit stellt sich die Frage, ob die genannten Laufzeiten zumindest teilweise gegen Europarecht verstoßen. Denn gemäß Art. 32 Abs. 1 VO (EG) 1107/2009 wird die Geltungsdauer einer Zulassung für einen Zeitraum von höchstens einem Jahr nach Ablauf der „in dem Pflanzenschutzmittel enthaltenen Wirkstoffe“ festgelegt und danach für so lange, wie der Wirkstoff genehmigt ist. Auch wenn Pflanzenschutzmittel in der Vergangenheit in der Regel für zehn Jahre zugelassen wurden, ${ }^{284}$ ist jedenfalls seit Inkrafttreten der VO (EG) 1107/2009 ab dem 14. Juni 2011

282 S.o. C I.3.b).

283 BVL, Online-Datenbank Pflanzenschutzmittel, Standardsuche (Wirkstoff: „Glyphosat“), abrufbar unter: https://apps2.bvl.bund.de/psm/jsp/ListeMain.jsp?page=1 (05.04.2019). Die Daten der jeweils letzten Zulassung ergeben sich aus BVL, Übersichtsliste der zugelassenen Pflanzenschutzmittel in Deutschland zum 03.04.2019, S. 4-82, abrufbar unter: www.bvl.bund.de/infopsm (15.06.2019).

284 BVL (Hrsg.), BVL-Report 13.1, Berichte zu Pflanzenschutzmitteln, Jahresbericht Pflanzenschutz-Kontrollprogramm 2017, 2018, S. 1, 16. 
(Art. 84) die Zulassungsdauer eines Mittels an die Dauer der EU-Wirkstoffgenehmigung gekoppelt. Wie gezeigt, ist grundsätzlich nur ein Übergangsjahr von der Wirkstoffneugenehmigung bis zur Neuzulassung durch die Mitgliedstaaten vorgesehen (Art. 43 Abs. 5 VO [EG] 1107/2009). Ferner gibt es eine Aufbrauchfrist von maximal sechs Monaten für den Verkauf und einem Jahr u.a. für den Verbrauch, sofern das Mittel nicht gesundheits- oder umweltschädlich ist (Art. 20 Abs. 2, Art. 46 VO [EG] 1107/2009). Es kommen aber eine Reihe anderer Erklärungen für die Fortdauer von Einzelzulassungen über den 15. Dezember 2018 hinaus in Betracht. Eingreifen könnten

- der Sonderfall des Art. 43 Abs. 6 VO (EG) 1107/2009 (Verlängerung bestehender Zulassungen wegen Verfahrensverzögerung seitens der Behörden),

- die Notfallklausel (Art. 53 VO [EG] 1107/2009; $\$ 29$ Abs. 1 Nr. 1 PflSchG) oder

- Übergangsrecht für Altzulassungen nach früherem Recht (Art. 80 und für den Parallelhandel Art. 52 Abs. 6 VO [EG] 1107/2009; \74 PflSchG).

Das BVL selbst erklärte im Dezember 2018, dass bis zum Stichtag für die Antragstellung auf Neuzulassung (15. März 2018) in Deutschland 37 Pflanzenschutzmittel mit dem Wirkstoff Glyphosat zugelassen seien. ${ }^{285}$ Da Anfang 2019, wie gezeigt, aber insgesamt nach Pflanzenschutzmittelliste 107 glyphosathaltige Mittel in Deutschland zugelassen waren, meint die Anzahl 37 offenbar nur diejenigen Mittel, die originär von deutschen Behörden für den Verkehr zugelassen wurden, also nicht etwa auf einer Genehmigung für den Parallelhandel (Art. 52 VO [EG] 1107/2009) in Deutschland beruhen. Für 28 dieser Mittel seien laut BVL - rechtzeitig (?) - Anträge auf Neuzulassung gestellt worden, was allerdings früheren Pressemeldungen widerspricht. ${ }^{286}$ Für 8 Mittel seien Zulassungen noch nach dem alten Pflanzenschutzgesetz erteilt worden, die über Dezember 2018 hinausreichten. Diese letzteren Zulassungen würden ohne erneuten Zulassungsantrag überprüft. Soweit es die 28 vorliegenden Anträge auf Neuzulassung betrifft, gelangte das BVL zu dem Schluss, dass der europaweit festgelegte Verfahrensablauf und dessen zeitliche Vorgaben es nicht zuließen, die Neuzulassung bis zum Fristablauf (15. Dezember 2018) zu erreichen. Teilweise fehle es, sofern Deutschland die Bewertungen vornehme (in zehn der 28 Antragsverfahren), an Einvernehmenserklärungen des Umweltbundesamtes. Teilweise fehle es an Erstbewertungen durch andere Mitgliedstaaten, die der BVL-Entscheidung vorausgehen müssten. Damit fielen alle Antragsfälle in den Anwendungsbereich des Art. 43 Abs. 6 VO (EG) 1107/2009. Das heißt, weil das Verfahren nicht in der vorgeschriebenen Zeit beendet werden könne und der Antragsteller dies nicht zu verantworten habe, müsse die bestehende Zulassung verlängert werden. Man habe daher „die be-

285 Auch zum Folgenden Presseinformation des BVL vom 11.12.2018 („Erneuerung der Zulassungen glyphosathaltiger Pflanzenschutzmittel noch nicht abgeschlossen“), abrufbar unter: https://www.bvl.bund.de/ (15.06.2019).

286 FAZ Nr. 259 vom 07.11.2018, S. 19 („Gerangel um Glyphosat geht in die nächste Runde“) erwähnt drei Anträge, die dem BVL vorgelegen hätten. 
stehenden Zulassungen glyphosathaltiger Pflanzenschutzmittel um ein Jahr bis zum 15. Dezember 2019“" verlängert. ${ }^{287}$

Inzwischen wurden offenbar weitere Verlängerungsentscheidungen dieser Art getroffen, woraufhin es zum öffentlichen Schlagabtausch zwischen dem UBA und dem BVL kam. Denn das UBA hatte, soweit es sein obligatorisches Einvernehmen gegenüber dem BVL schon erteilt hatte, Anwendungsbestimmungen zugunsten der Umwelt verlangt, namentlich die Vorhaltung von Blühflächen und Brachen durch die glyphosatnutzenden Betriebe. Das BVL ließ die geforderten Beschränkungen aber leerlaufen, indem es argumentierte, dass das Gesamtverfahren nicht fristgerecht abgeschlossen werden könne und deshalb gemäß Art. 43 Abs. 6 VO (EG) 1107/2009, $\$ 38$ PflSchG die bestehende Zulassung - ohne neue Beschränkungen - von Amts wegen verlängert werden müsse. Ob das BVL damit rechtswidrig handelte, wie der Fachgebietsleiter für Pflanzenschutzmittel des UBA es als mögliche Interpretation in den Raum stellte, ${ }^{288}$ ist allerdings die Frage. Denn das eigentliche Problem liegt in der Konzeption des Art. 43 Abs. 6 VO (EG) 1107/2009, der die Option zur Verlängerung ohne jeden Hinweis auf die Möglichkeit beschränkender Auflagen im Sinne zwischenzeitlicher Entwicklungen eröffnet. Während im Recht der Europäischen Union ansonsten gilt, dass Haushaltsprobleme oder eine mangelhafte Ausstattung der Behörden in den Mitgliedstaaten kein Grund dafür sind, Pflichten nicht zu erfüllen oder Fristen nicht einzuhalten, setzt die genannte Vorschrift diese Grundregel gerade bei der Pestizidzulassung offen außer Kraft. Denn die Bestimmung schließt allenfalls Verlängerungen aus, die für den Abschluss der Prüfung und eine Erneuerungsentscheidung nicht mehr „notwendig“ sind. Da den Mitgliedstaaten bei der Beurteilung dieser Voraussetzung aber ein großer Beurteilungsspielraum zugestanden werden dürfte, wird diese Grenze wohl nur in krassen Fällen der Verschleppung durch die Behörden oder der Gewährung extrem langer Verlängerungslaufzeiten überschritten. Das vorprogrammierte und hier auch eingetretene Ergebnis der Schlupflochregelung aus Art. 43 Abs. 6 VO (EG) 1107/2009 liegt offen zutage: Ein hoch- bis überkomplexer Verfahrensablauf mit zu vielen Beteiligten dient als Rechtfertigung dafür, Verlängerungsentscheidungen auf der Basis einer Altzulassung zu treffen und unter Umständen auch diese wieder zu verlängern. So wird es möglich, alle Vorgaben aus der schon geltenden neuen Glyphosat-Wirkstoffgenehmigung zu unterlaufen, obwohl diese ohnehin kaum über vage Aufforderungen an die Mitgliedstaaten hinausreichen. Im Ergebnis haben die Auflagen zum Schutz von Umwelt und Gesundheit auch eineinhalb Jahre nach der Wirk-

$287 B V L$, Presseinformation vom 11.12.2018, (Fn. 285). Dass Verlängerungen zunächst für 25 glyphosathaltige Pestizide erteilt wurden, deren Neuzulassung beantragt war, ergibt sich aus der Antwort der Bundesregierung zur Kleinen Anfrage der Abgeordneten Harald Ebner u.a. und der Fraktion Bündnis90/Die Grünen, BT-Drs. 19/7739, S. 4 (zu Frage 4).

288 Siebert, Pflanzenschutzmittel: Streit um die Verlängerung von Zulassungen, Deutschlandfunk vom 02.04.2019, www.deutschlandfunk.de (15.06.2019): „Jörn Wogram vom UBA kritisiert das BVL dafür: "Und zwar hat es die Zulassung von Mitteln neu ausgesprochen, begrenzt für ein Jahr, aber obne eine Anwendungsbestimmung zum Schutz der Umwelt, die das UBA mit seinem Einvernehmen zur Zulassung verbunden hatte. Jetzt kann man sich darüber streiten, inwieweit das dann ein rechtskonformes Vorgehen ist, denn eigentlich wäre das BVL an das Votum des UBA streng gebunden.". 
stoff-Neugenehmigung noch keinerlei Auswirkungen auf die Wirklichkeit der Pestizid-Zulassung entfalten können.

Soweit die Zulassungsdauer bei einzelnen Pflanzenschutzmittel auch ohne Verlängerung schon über 2018 hinausläuft, beruft sich das BVL darauf, dass diese Zulassungen noch nach dem alten Pflanzenschutzgesetz erteilt worden seien. In der Tat dürfen gemäß \74 Abs. 2 PflSchG Pflanzenschutzmittel, die vor dem 14. Februar 2012 zugelassen worden sind, noch in Verkehr gebracht werden, bis ihre Zulassung durch Zeitablauf endet, es sei denn, die Zulassung endet zu einem früheren Zeitpunkt durch Widerruf oder Rücknahme. Das korrespondiert allerdings nicht mit den Daten der insoweit auffälligen glyphosathaltigen Mittel. Denn, wie gezeigt, wurden „Roundup REKORD“ und „MON 9991-SG “, die bis Ende 2024 gelten, zuletzt am 25.3.2014 bzw. 3.4.2014 zugelassen, „Excel DF Gold“ und „Vorox Unkrautfrei direkt“, deren Laufzeit bis Ende 2026 reicht, wurden am 2.5.2016 bzw. 1.2.2017 zuletzt zugelassen. Die deutsche Übergangsbestimmung ( $\$ 74$ Abs. 2 PflSchG) erfasst diese Mittel jedenfalls nicht, da sie nach dem in ihr genannten Stichtag (14. Februar 2012) zugelassen wurden. Zudem erscheint fraglich, ob die Anforderungen der maßgeblichen europäischen Übergangsbestimmungen (Art. 80 Abs. 5 und für den Parallelhandel Art. 52 Abs. 6 VO [EG] 1107/2009) erfüllt wurden. Eine abschließende Bewertung ist hier aber nicht möglich.

Eine Pflicht zur Aufhebung noch laufender Zulassungen für Pflanzenschutzmittel hätte Deutschland nur, wenn es z.B. nach dem Auslaufen der aktuellen GlyphosatGenehmigung Mitte Dezember 2022 keine weitere Genehmigung für diesen Wirkstoff mehr geben sollte. ${ }^{289} \mathrm{Da}$ der Wirkstoff aber gerade wiedergenehmigt wurde, kommt diese Aufhebungsverpflichtung momentan nicht zum Zuge, - selbst wenn Deutschland in einigen Fällen die europarechtliche Pflicht zur Neuzulassung glyphosathaltiger Pflanzenschutzmittel leerlaufen lassen sollte. Denkbar wäre, dass sich die deutschen Behörden insoweit auf den Standpunkt stellen, es sei ja kein Antrag von den betreffenden Herstellern der Mittel auf Neuzulassung gestellt, während diese wiederum argumentieren dürften, sie seien doch im Besitz einer noch laufenden Genehmigung, d.h. eines begünstigenden Verwaltungsakts. Hier steckt womöglich ein Fehler im System, d.h. es fehlt eine Regelung, die sicherstellt, dass die Einzelfallgenehmigung zugunsten eines Pflanzenschutzmittels spätestens ein Jahr nach Ablauf des in ihm enthaltenen Wirkstoffs ebenfalls abläuft.

\section{b) Vorstöße zur Einschränkung der Nutzung glyphosathaltiger Mittel im EU-weiten Vergleich}

Die Entscheidung über die Wiedergenehmigung des Wirkstoffs Glyphosat war entsprechend den konträren Positionen der Mitgliedstaaten denkbar knapp ausgefallen. Neun Mitgliedstaaten hatten im Berufungsausschuss gegen die erneute Genehmigung

289 Art. 44 Abs. 3 lit. a i.V.m. Art. 29 Abs. 1 lit. a VO (EG) 1107/2009. S.a. \39 Abs. 1 Nr. 1 PflSchG. 
von Glyphosat gestimmt. Die schwedische Regierung hatte sich als einzige schon vom Ergebnis der Risikobewertung distanziert. ${ }^{290}$

Schon vor der entscheidenden Abstimmung vom Dezember 2017 hatte die französische Regierung ihren Willen bekundet, die Nutzung von glyphosathaltigen Pflanzenschutzmitteln in Frankreich sukzessive zu verbieten. Ein Verbot besteht hier bereits für alle öffentlich zugänglichen Plätze seit Januar 2017, ein Verbot der privaten Nutzung war ursprünglich für Januar 2019, ein vollständiges Verbot ist ab 2022 geplant. ${ }^{291}$ Erheblicher Widerstand aus der Landwirtschaft bremste allerdings die Umsetzung dieser Pläne.

Die niederländische Landwirtschaftsministerin Carola Schouten hatte zwar die erneute Genehmigung des Wirkstoffs Glyphosat in Brüssel unterstützt, kündigte aber nach der Entscheidung an, sich dafür einsetzen, in den kommenden fünf Jahren die Entwicklung von Alternativen voranzutreiben. Denn in den Niederlanden wird generell eine Senkung der Umweltbelastung durch chemische Pflanzenschutzmittel angestrebt. Im Unterschied dazu hätte die spanische Regierung eine Verlängerung der Glyphosatgenehmigung für weitere fünfzehn Jahre bevorzugt. ${ }^{292}$

Österreich hatte in sämtlichen EU-Gremien gegen die weitere Zulassung von Glyphosat gestimmt. ${ }^{293}$ Innerstaatlich ist seit 2013 die Sikkation weitestgehend verboten. ${ }^{294}$ Die Behandlung von Erntegut, das der Herstellung von Lebens- oder Futtermitteln dient, wurde verboten, um Glyphosatrückstände in der Nahrungsmittelkette einzudämmen. Zudem hat der Bund die Bundesländer ermächtigt, Einschränkungen und Verbote bei der Verwendung von Pflanzenschutzmitteln unter bestimmten Bedingungen oder in bestimmten Gebieten zu erlassen. ${ }^{295}$

In Deutschland hatte die Große Koalition zwar den zeitlich gestreckten Ausstieg aus der Glyphosatnutzung im Koalitionsvertrag vereinbart, einer der Koalitionspartner aber dann eigenmächtig die erneute Genehmigung von Glyphosat für weitere fünf Jahre in Brüssel entscheidend mitbewirkt. ${ }^{296}$ Allerdings hat sich die Forderung nach dem Ausstieg bzw. der „vernünftigen Einschränkung“ damit nicht erledigt, wie zahl-

290 S.o. B I.2.b) und C I.3.a).

291 Gouvernement français, Verlautbarung vom 26.09.2017 („Glyphosate: il n’y aura pas d'interdiction brutale mais une élimination progressive des pesticides qui présentent un risque pour la santé et ou la biodiversité“), abrufbar unter: www.gouvernement.fr (15.06.2019).

292 Awater-Esper, Glyphosat: So reagieren andere EU-Mitgliedstaaten, „top agrar online“ vom 04.12.2017, abrufbar unter: www.topagrar.com (15.06.2019).

293 Ranzenmayr, S. 44 mit Anm. 90.

294 Pflanzenschutzgesetz (PMG) 2011, (österr.) BGBl. I 143/2013.

295 Ranzenmayr, S. $47 \mathrm{f}$.

296 S.o. B I.2.b). 
reiche Vorstöße im Bundestag und Bundesrat zeigen. ${ }^{297}$ Wie in vielen anderen EUMitgliedstaaten sind auch in Deutschland gentechnisch veränderte Kulturpflanzen grundsätzlich nicht zum Anbau zugelassen, ${ }^{298}$ so dass die damit verbundenen Probleme hier allenfalls im Kontext des Exports (z.B. Einfuhr von Soja aus Argentinien) eine Rolle spielen. Bemerkenswert ist in Deutschland allerdings der Umgang mit dem Gebrauch glyphosathaltiger Mittel durch nichtprofessionelle Verwender und Verwenderinnen. Denn obwohl die Einschränkung der Nutzung durch Amateure ein wesentliches Argument für die Zustimmung des Bundeslandwirtschaftsministers zur Wiedergenehmigung von Glyphosat gewesen sein soll, ist auf diesem Felde innerstaatlich nichts geschehen. Nach wie vor gilt gemäß $\ 12$ Abs. 3 Satz 2 PflSchG, dass im Haus- und Kleingartenbereich nur Pflanzenschutzmittel angewandt werden dürfen, die für die Anwendung durch nichtberufliche Anwender zugelassen sind oder deren Eignung für diesen Bereich speziell festgestellt wurde. Einer parlamentarischen Anfrage zu diesem Bereich wich die Bundesregierung mit der Auskunft aus, dass Pflanzenschutzmittel für den Haus- und Kleingarten, die den Aufdruck „Anwendung durch nichtberufliche Anwender zulässig“ trügen, von jedem erworben und angewandt werden könnten. ${ }^{299}$ Dass das nach wie vor auch glyphosathaltige Mittel betrifft, muss unter diesen Umständen wohl angenommen werden. Sämtliche Fragen danach, wie weit die im Koalitionsvertrag vereinbarte Reduktionsstrategie („systematische Minderungsstrategie") für Glyphosat gediehen sei, werden mit einem Hinweis auf noch nicht abgeschlossene Beratungen auf Ressortebene beschieden. Nachdem die erneute Wirkstoffgenehmigung für Glyphosat eineinhalb Jahre zurückliegt, ist ledig-

297 Siehe Antrag der Fraktion Bündnis 90/Die Grünen („Glyphosatausstieg jetzt einleiten“) vom 11.12.2017, BT-Drs. 19/230; Antrag der SPD-Fraktion vom 11.12.2017 („Nationaler Ausstieg aus der Anwendung von glyphosathaltigen Pflanzenschutzmitteln“), BT-Drs. 19/232; Antrag der Abgeordneten Dr. Kirsten Tackmann u.a. und der Fraktion „Die Linke“(„Glyphosathaltige Pflanzenschutzmittel in Deutschland verbieten“) vom 11.12.2017, BT-Drs. 19/226. Für eine beschränkte Weiternutzung: Antrag der Abgeordneten Christian Lindner u.a., BT-Drs. 19/216, (Fn. 36). Anträge im Bundesrat zur Eindämmung des Glyphosatgebrauchs: Entschließungsantrag des Landes Thüringens vom 06.12.2017, BR-Drs. 740/17; Entschließungsantrag des Saarlandes ("Schutz der biologischen Vielfalt durch die Beschränkung von glyphosathaltigen Pflanzenschutzmitteln”) vom 13.12.2017, BR-Drs. $762 / 17$.

298 Siehe dazu die Richtlinie (EU) 2015/412 des Europäischen Parlaments und des Rates vom 11. März 2015 zur Änderung der Richtlinie 2001/18/EG zu der den Mitgliedstaaten eingeräumten Möglichkeit, den Anbau von gentechnisch veränderten Organismen (GVO) in ihrem Hoheitsgebiet zu beschränken oder zu untersagen („Opt-out-Richtlinie“), ABl. (EU) L 68 v. 13.03.2015, S. 1. Aufgrund des hierdurch neu eingefügten Art. 26 b kann ein Mitgliedstaat veranlassen, dass sein Hoheitsgebiet von der Zulassung eines bestimmten gentechnisch veränderten Organismus ausgenommen wird. Davon hat Deutschland Gebrauch gemacht. S. Wissenschaftlicher Dienst des Deutschen Bundestages, Ausarbeitung PE 6 - 3000 - 31/17: Vorgaben und Umsetzung der Richtlinie (EU) 2015/412 vom 20.06.2017.

299 BReg, BT-Drs. 19/4097 vom 28.08.2018, (Fn. 24), S. 3. 
lich geplant, die noch unbekannte Strategie der Reduktion in eine „Fünfte Verordnung zur Änderung der Pflanzenschutz-Anwendungsverordnung " einzubringen. ${ }^{300}$

\section{c) Vereinbarkeit von „Reduktionsstrategien" (Verboten) mit den europäischen Vorgaben zur Neuzulassung glyphosathaltiger Pflanzenschutzmittel}

Wie gezeigt, haben die Mitgliedstaaten nur sehr eingeschränkte Möglichkeiten, die obligatorische Neuzulassung eines Pflanzenschutzmittels zu versagen, das einen erneut genehmigten Wirkstoff wie z.B. Glyphosat enthält. Denn grundsätzlich kann die Neuzulassung nur daran scheitern, dass ein einzelnes Anwendungsmittel entweder die Voraussetzungen des Art. 29 VO (EG) 1107/2009 nicht mehr erfüllt (Art. 43 Abs. 1 VO [EG] 1107/2009) oder neue Bedingungen und Einschränkungen aus der Verordnung zur Erneuerung der Genehmigung des Wirkstoffs nicht erfüllt sind (Art. 43 Abs. 3 VO [EG] 1107/2009). Eine Berufung auf ortsspezifische Eigenheiten zum Zwecke der landesweiten Verweigerung der Zulassung (Art. 36 Abs. 3 VO [EG] 1107/2009) kommt in Betracht, wenn Änderungen in diesem Bereich eingetreten sind. 301

Aus dem Sinn und Zweck des gestuften Zulassungsverfahrens wie der geteilten $\mathrm{Zu}$ ständigkeiten folgt, dass die einzelnen Mitgliedstaaten bei der erneuten Zulassung von Pflanzenschutzmitteln keinen Aspekt erneut der kritischen Prüfung unterziehen dürfen, der schon Gegenstand der Bewertung im Rahmen der Wirkstoff- Neugenehmigung 302 oder der zonalen Bewertung bei der Pflanzenschutzmittel-Neuzulassung (Art. 43 Abs. 3 UAbs. 2 VO [EG] 1107/2009) gewesen war. Sie dürfen also vorangegangene Bewertungen, die dafür zuständige Stellen getroffen haben, nicht desavouieren. Das schließt allerdings nicht aus, dass konkrete Auswirkungen eines bestimmten Anwendungsprodukts in einer bestimmten Region bei den vorhandenen Bewertungen nicht berücksichtigt wurden oder sich Änderungen ergeben haben, so dass es sich nicht mehr um denselben Prüfgegenstand handelt. Dann dürfen bzw. müssen die Mitgliedstaaten handeln. Um einen „nationalen Sonderweg“ zu rechtfertigen, müssen grundsätzlich besondere landestypische Umstände vorliegen, und genügt eine eigenständige Schutzpolitik der Mitgliedstaaten allein nicht. ${ }^{303}$ Leitend sind auch insoweit die „einheitlichen Grundsätze“ i.S.v. Art. 29 Abs. 6 VO (EG) 1107/2009 für die Bewertung und Zulassung von Pflanzenschutzmitteln, wie sie in Teil I der Durchführungsverordnung (EU) 546/2011 niedergelegt sind, hier im Besonderen in Abschnitt C, Nr. 1.2.:

300 BReg, BT-Drs. 19/4097 vom 28.08.2018, (Fn. 24), S. 4 f., 6. Zu den Planungen der Bundesumweltministerin Schulze (10\% Freifläche für Insekten bei glyphosatnutzenden Betrieben ab 2020, Verbot der Nutzung für Privatgärten und öffentliche Flächen), FAZ Nr. 259 vom 07.11.2018, S. 19 („Gerangel um Glyphosat geht in die nächste Runde“).

301 S.o. C II.1.c).

302 So wohl auch der Wissenschaftliche Dienst des Deutschen Bundestages, Die Möglichkeit nationaler Verbote von Glyphosat - Die Rechtslage nach der erneuten Zulassung auf Unionsebene, Gutachten vom 12.12.2017, Az. PE 6-3000 - 95/17, S. 8, 19.

303 So auch Wissenschaftlicher Dienst des BT, (Fn. 302), S. 13. 
„Die Mitgliedstaaten stellen sicher, dass bei Zulassungsentscheidungen erforderlichenfalls die Bedingungen in den vorgesehenen Verwendungsregionen in Bezug auf Landwirtschaft, Pflanzenschutz und Umwelt - einschließlich Klima - berücksichtigt werden. Diese Erwägungen können dazu führen, dass besondere Verwendungsbedingungen und-beschränkungen festgelegt werden, und dass die Zulassung gegebenenfalls nur für bestimmte Gebiete innerbalb des betreffenden Mitgliedstaats gewährt wird."

Diese Formulierung lässt zwar die Tendenz erkennen, die Zulassung möglichst zu bewilligen - nötigenfalls auf der Basis regionaler Differenzierung. Dem scheint auch die Annahme zugrunde zu liegen, dass sich spezifische Verwendungsbedingungen immer nur auf einzelne Regionen beziehen und nicht mit den Staatsgrenzen koinzidieren. Eine landesweite Verweigerung der Zulassung bestimmter Mittel auf der Basis des Art. 36 Abs. 3 VO (EG) 1107/2009 kann dies aber nicht ausschließen, vorausgesetzt, dass diese Norm anwendbar und ihre Voraussetzungen erfüllt sind.

Darüber hinaus können sich die Mitgliedstaaten bei der Anwendung einschränkender Maßnahmen und Strategien immer auch auf das Vorsorgeprinzip stützen. Nach Art. 1 Abs. 4 Satz 2 VO (EG) 1107/2009 ist es ihnen insbesondere ,freigestellt“, das Vorsorgeprinzip anzuwenden, wenn wissenschaftliche Ungewissheit besteht, ob die in ihrem Hoheitsgebiet „zuzulassenden“ Pflanzenschutzmittel Gefahren für die Gesundheit von Mensch oder Tier oder die Umwelt bergen. Da das Vorsorgeprinzip ein bindendes Rechtsprinzip und keine bloße Option ist, ${ }^{304}$ muss die Formulierung so verstanden werden, dass ihre Bedeutung über die selbstverständliche Geltung des Vorsorgeprinzips bei allen Bewertungen hinausreicht. In der Tat sprechen Konzeption und systematische Stellung des Art. 1 Abs. 4 Satz 2 VO (EG) 1107/2009 dagegen, ihn als eigenständige Ermächtigungsgrundlage anzusehen. Deshalb können allein auf dieser Grundlage Pflanzenschutzmittel weder verboten noch sonst beschränkt werden. ${ }^{305}$ Nehmen die Mitgliedstaaten aber ihre anderweitig bestehenden Kompetenzen im Rahmen der (Neu-)Zulassung von Pflanzenschutzmitteln wahr, verleiht ihnen Art. 1 Abs. 4 Satz 2 VO (EG) 1107/2009 ein zusätzliches Maß an Bewertungsmacht, nämlich dann wenn wissenschaftliche Ungewissheit besteht. Art. 1 Abs. 4 Satz 2 VO (EG) 1107/2009 könnte in letzter Konsequenz so verstanden werden, dass er die Mitgliedstaaten von der zuvor beschriebenen Grundregel entbindet, dass sie bei der erneuten Zulassung bestimmter Pflanzenschutzmittel keinen Aspekt erneut der kritischen Prüfung unterziehen dürfen, der schon Gegenstand vorangehender Bewertungen gewesen war. Weniger weit verstanden könnte er die Neubewertung zumindest erlauben, wenn Ungewissheit sich durch neue wissenschaftliche Erkenntnisse weiter in Richtung Gefahr verstärkt hat oder nicht völlig klar ist, ob schädliche Auswirkungen mit dem Wirkstoff oder der Art der Formulierung in einem Pflanzenschutzmittel zusammenhängen. Für ein solches Verständnis des Art. 1 Abs. 4 Satz 2 VO (EG) 1107/2009 spricht, dass die Mitgliedstaaten für die Sicherheit der Anwen-

304 S.u. E I.

305 Wissenschaftlicher Dienst des BT, (Fn. 302), S. 16. 
dungsprodukte zuständig sind und die Verantwortung vor Ort den Menschen gegenüber tragen. Es ist ein Verständnis, das auch dem Subsidiaritätsprinzip entspricht.

Konkret stellt sich z.B. die Frage, ob die Mitgliedsstaaten die Verwendung glyphosathaltiger Mittel durch Amateure im heimischen Hof, Beet und Garten gesetzlich verbieten dürfen. Nach den Regelungen der Durchführungsverordnung (EU) 2017/2324 zur Glyphosat-Neugenehmigung und den in Bezug genommenen Dokumenten „achten“ die Mitgliedstaaten bei der Anwendung der „einheitlichen Grundsätze“ gemäß Art. 29 Abs. 6 VO (EG) 1107/2009 auf „den Schutz gewerblicher und nichtgewerblicher Verwender". Die Durchführungsverordnung gibt zwar vor, dass die Anwendungsbedingungen ,gegebenenfalls Maßnahmen zur Risikobegrenzung umfassen" müssen, aber keine klaren Anhaltspunkte, wie dieser Schutz auszusehen hat. Insoweit gilt zwar die generelle Zielvorgabe der VO (EG) 1107/2009, „ein hohes Schutzniveau für die Gesundheit von Mensch und Tier und für die Umwelt" zu gewährleisten (Art. 1 Abs. 3 und Erwägungsgrund 8 der Verordnung [EG] 1107/2009). Die entscheidende Frage ist aber, ob der Schutz nicht-professioneller Anwender und Anwenderinnen nur „bei der Anwendung der einheitlichen Grundsätze“ zum Zuge kommt, wie es die Durchführungsverordnung (EU) 2017/2324 an dieser Stelle ausdrückt. Sieht man in die im Anhang der Durchführungsverordnung (EU) 546/2011 aufgelisteten einheitlichen Grundsätze hinein, fällt insoweit Teil I, C, Nr. 2.4.1.3 ins Auge. Danach sind für Pflanzenschutzmittel, die aufgrund ihrer Eigenschaften oder bei unsachgemäßer Handhabung oder Verwendung sehr gefährlich sein können, „besondere Beschränkungen in Bezug auf Verpackungsgröße, Art der Formulierung, Vermarktung sowie Verwendungsweise und -bedingungen aufzuerlegen". Solche Mittel dürfen „nicht für eine Verwendung durch nichtgewerbliche Verwender zugelassen werden, wenn sie eingestuft sind als ... "Die folgende Auflistung beinhaltet die Eigenschaften „akut toxisch der Kategorien 1 und 2“ (höchste Gefahrenklassen) sowie in den verschiedenen Aufnahmeformen organtoxisch (STOT: specific target organ toxicity) der Kategorie 1 bei einmaliger Exposition sind. Soweit diese Gefahrklassen nicht erreicht werden, was mit Blick auf die offiziellen Bewertungsergebnisse zum Wirkstoff Glyphosat hier wohl angenommen werden muss, greift die Verpflichtung zur Sperrung des privaten Gebrauchs nicht ein. Ob die Mitgliedstaaten den privaten Gebrauch glyphosathaltiger Produkte dann aber gleichwohl generell unterbinden dürfen, ist die interessante Frage. An sich fällt die private Nutzung unter den Begriff der „Verwendungsweise und -bedingungen“, kann also reguliert werden. Unproblematisch ist es in jedem Falle, privaten Nutzern und Nutzerinnen Beschränkungen in Bezug auf die Verpackungsgröße u.s.w. aufzuerlegen. Mitgliedstaaten, die darüber hinaus ein totales Verbot der privaten Nutzung anstreben, könnten argumentieren, dass die private Nutzung glyphosathaltiger Mittel im betreffenden Land unzuträglich ist und die drohenden Gefahren nicht durch risikomindernde Maßnahmen ausgeschlossen werden können. Insoweit muss ihnen auch ein weiter Beurteilungsspielraum zugestanden werden, weil nur sie die Verhältnisse vor Ort kennen und der private Umgang mit glyphosathaltigen Pestiziden nicht Teil der europäischen oder zonalen Bewertungsprozesse gewesen ist. Es ist aber schwierig, das Verhalten oder die Gefährdung von Privatpersonen als landesspezifische Eigenart darzustellen. Da- 
gegen könnten die Mitgliedstaaten unter Bezugnahme auf Art. 1 Abs. 4 Satz 2 VO (EG) 1107/2009 argumentieren, dass in Bezug auf das Ausmaß des Risikos und die Möglichkeiten, es durch risikomindernde Maßnahmen wirksam zu bekämpfen, wissenschaftliche Ungewissheit besteht, Gefahren in diesem Bereich besonders wahrscheinlich sind und nur das Verbot der privaten Nutzung das gebotene „hohe Schutzniveau für die Gesundheit“ (Art. 1 Abs. 3 Verordnung [EG] 1107/2009) herstellen kann.

Eine weitere Forderung richtet sich auf das Verbot der Sikkation, wie es z.B. Österreich schon weitestgehend umgesetzt hat. ${ }^{306}$ Unzweifelhaft verweist die Durchführungsverordnung für die erneute Genehmigung von Glyphosat auf eine Fülle von Anforderungen und „Endpoints“, die für Lebens- und Futtermittel sowie die einzelnen Agrarprodukte maximale Rückstandsmengen für Glyphosat und AMPA festlegen. ${ }^{307}$ Die einheitlichen Grundsätze im Anhang der DurchführungsVO (EU) 546/2011 machen in Teil I, B, Nr. 2.4.2. detaillierte Vorgaben für die Bewertung, soweit es gesundheitliche Auswirkungen von Rückständen eines Pflanzenschutzmittels betrifft. Gemäß Nr. 2.4.2.5. bewerten die Mitgliedstaaten „mit Hilfe eines geeigneten Berechnungsmodells die potenzielle Exposition der Verbraucher über die Nahrung oder, soweit dies relevant ist, andere Expositionswege" und berücksichtigen dabei auch sonstige Rückstandsquellen. Vor der Bewertung prüfen sie gemäß Nr. 2.4.2.2. „Angaben über die vorgeschlagene gute landwirtschaftliche Praxis einschließlich der im Anhang der Verordnung (EU) Nr. 545/2011 genannten Angaben über die Anwendung und die vorgeschlagenen Sicherheitswartezeiten bei den vorgesehenen Verwendungszwecken sowie Angaben über Rückhalte- oder Lagerfristen bei Verwendung nach der Ernte“. Teil I, C, Nr.2.4.2.1. gibt den Mitgliedstaaten auf, bei Zulassungen „sicherzustellen, dass die Rückstände von den Mindestmengen des Pflanzenschutzmittels stammen, die zu einer angemessenen Bekämpfung gemäß guter landwirtschaftlicher Praxis erforderlich sind“, und dass „die Verwendungsbedingungen (Wartezeiten, Lagerfristen und Fristen vor der Ernte) die Rückstände bei der Ernte, der Schlachtung oder gegebenenfalls nach der Lagerung so gering wie möglich halten“. Soweit Rückstandshöchstgehalte (MRLs) festgelegt wurden, müssen Antragsteller nachweisen, dass ihre Produkte sie auch erfüllen. Dabei muss der schlimmstmögliche Fall einer Exposition von Verbrauchern zugrunde gelegt werden, der aber gleichwohl „auf der guten landwirtschaftlichen Praxis“ beruht (Teil I, C, Nrn. 2.4.2.2.-2.4.2.4.). All diese Vorgaben laufen im Grunde darauf hinaus, bei der zonalen Bewertung wie auch bei der konkreten Zulassungsentscheidung für die Einhaltung (mehr oder weniger) „unbedenklicher“ Höchstrückstandsmengen zu sorgen, um die Zulassung von Pestiziden zu ermöglichen. Die Hinweise auf einzuhaltende Fristen vor der Ernte lassen die Wertung erkennen, dass die Nutzung glyphosathaltiger Mittel zur Sikkation nicht schon per se als Verstoß gegen die gute landwirtschaftliche Praxis anzusehen ist. Allerdings bindet das dem einzelstaatlichen Gesetzgeber, der die Nutzung zur Sikkation generell verbieten will, nicht völlig die Hände. 
Zum einen könnte er zeigen, dass im betreffenden Land eine besondere Rückstandsproblematik besteht, die mit dieser Nutzung zusammenhängt und mit risikomindernden Maßnahmen nicht in den Griff zu bekommen ist. Zum anderen bestehen auf diesem Feld wissenschaftliche Ungewissheiten, die dem Mitgliedstaat einen weiten Beurteilungsspielraum hinsichtlich der Frage einräumt, inwieweit das Vorsorgeprinzip (Art. 1 Abs. 4 Satz 2 VO [EG] 1107/2009) gebietet, die Verwendung glyphosathaltiger Mittel zur Sikkation von der Zulassung auszunehmen.

Die Frage, ob ein Verbot glyphosathaltiger Pflanzenschutzmittel im Hinblick auf deren Auswirkungen auf die Biodiversität zulässig wäre, unterscheidet sich von den beiden vorgenannten Fällen. Denn die Biodiversität hängt in ungleich stärkerem Maße mit den konkreten örtlichen ökologischen und landwirtschaftlichen Verhältnissen zusammen. Schon in den Bewertungsberichten zur Glyphosat-Wiedergenehmigung wird betont, dass es kritische Auswirkungen auf die Biodiversität gibt und deshalb Maßnahmen ergriffen werden müssen. ${ }^{308}$ Weil die sogenannten „Nahrungsnetzeffekte" in den einzelnen Mitgliedstaaten bzw. Regionen unterschiedlich ausgeprägt sein können, liegt insoweit ein Handeln auf staatlicher Ebene im Rahmen der Pflanzenschutzmittelzulassung nahe. Dabei muss aber mit Blick auf die europäischen Vorga-

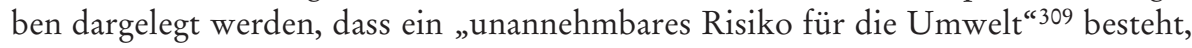
das nicht durch risikomindernde Maßnahmen aufgrund Art. 31 Abs. 2, 3 VO (EG) 2011/2009 bewältigt werden kann. ${ }^{310}$ Fraglich ist deshalb, ob ein einzelner Mitgliedstaat die Zulassung sämtlicher glyphosathaltiger Mittel etwa unter Berufung darauf verweigern bzw. insgesamt gesetzlich ausschließen könnte. Die „einheitlichen Grundsätze“ aus dem Anhang zur Durchführungsverordnung (EU) 546/2011 (Teil I, C, Nr. 1.2. ${ }^{311}$ scheinen insoweit davon auszugehen, dass bei kritischen Befunden zur Biodiversität „die Zulassung nur für bestimmte Gebiete gewäbrt" wird, wo das Umweltproblem (noch) nicht besteht. Das könnte im Umkehrschluss bedeuten, dass die Zulassung nur in Bezug auf tatsächlich belastete bzw. gefährdete Teilgebiete verweigert werden dürfte. Eine solche Tendenz zur Zulassung von Pestiziden scheint den einheitlichen Grundsätzen zugrunde zu liegen. Weil aber auch hier das Vorsorgeprinzip gilt (Art. 1 Abs. 4 Satz 2 VO [EG] 1107/2009), muss keinesfalls eine konkrete Gefahr bestehen, sondern kann und muss der Mitgliedstaat auch vorsorgende Schutzmaßnahmen ergreifen können. ${ }^{312}$ Außerdem können die auf Art. 29 Abs. 6 VO (EG) 2011/2009 fußenden einheitlichen Grundsätze nicht die Möglichkeit der Mitgliedstaaten überspielen, sich für ein landesweites Verbot auf Art. 36 Abs. 3 VO (EG) 2011/2009 zu berufen - vorausgesetzt, die Norm ist auch auf Erneuerungsverfahren anwendbar und ihre Voraussetzungen sind erfüllt. Ob ein Mitgliedstaat größere Teilgebiete ganz generell von Pestizideinflüssen freizuhalten versuchen kann, ist eine Frage der Schutzgebietsausweisung, die hier nicht vertieft werden soll.

308 Siehe z.B. RAR, (Fn. 172), S. 134.

309 Art. 43 Abs. 1 i.V.m. Art. 29 Abs. 1 lit. e, Art. 4 Abs. 3 lit. e (insbesondere iii) VO (EG) 2011/2009.

310 Wissenschaftlicher Dienst des BT, (Fn. 302), S. $9 \mathrm{f}$.

311 Siehe Zitat der Textstelle zu Beginn von C II.3.c).

312 Siehe i.E. u. E I. 
Soweit es das Ausbringen glyphosathaltiger Unkrautvernichtungsmittel auf öffentlichen Flächen wie Parks, Sport- und Freizeitplätzen, Schulgeländen, Kinderspielplätzen oder Gebieten in unmittelbarer Nähe von Gesundheitseinrichtungen betrifft, verlangt die Durchführungsverordnung zur Neugenehmigung von Glyphosat, dass die „Verwendung" von Mitteln, die es enthalten, lediglich „minimiert wird“.313 Diese Formulierung scheint auf eine Weiterverwendung auf - mehr oder weniger - niedrigstmöglichem Niveau zu zielen, schließt aber ein Verbot der Verwendung auf solchen Flächen auch nicht aus. Im Einzelnen stellen sich ähnliche Fragen wie zuvor.

Insgesamt zeigen die hier ausgewählten Beispiele, dass die meisten der politisch diskutierten Forderungen nach einem Ausstieg aus der Glyphosatnutzung rechtlich nur schwer realisierbar sind. Ein wesentliches Problem liegt darin, dass Mitgliedstaaten, die eine Glyphosatnutzung auch nur in bestimmten Zusammenhängen (z.B. Gebrauch durch Privatleute oder zur Sikkation) unterbinden wollen, darlegen müssen, dass das gerade in ihrem Land wegen dessen Eigenheiten, die sich auf die gesamte Fläche beziehen, erforderlich ist und risikomindernde Maßnahmen nicht genügen, um den erkannten Risiken vorzubeugen. Das ist aber kaum realistisch. Denn die insoweit bestehenden Bedenken - Eintragung von Glyphosat/AMPA in die Nahrungsmittelkette (Sikkation) oder unsachgemäße Nutzung durch Amateure - sind nicht auf die Gegebenheiten eines bestimmten Mitgliedstaats beschränkt. Nur dort, wo die wissenschaftliche Unsicherheit manifest ist, kann Art. 1 Abs. 4 Satz 2 VO (EG) 1107/2009 eine abweichende Gefahrbewertung durch den einzelnen Mitgliedstaat auch in solchen Aspekten tragen, in denen keine landestypischen Besonderheiten bestehen. Somit scheint die Durchführungsverordnung zur Neugenehmigung von Glyphosat zwar auf den ersten Blick Sicherheitsanforderungen zu beinhalten, die von den Mitgliedstaaten bei der obligatorischen Neuzulassung aller Anwendungsprodukte umgesetzt werden sollen. Die dabei zu beachtenden „einheitlichen Grundsätze“ der Bewertung i.S.v. Art. 29 Abs. 6 VO (EG) 2011/2009 sind aber ihrer Tendenz nach so formuliert, dass sie grundsätzlich die Zulassung, allenfalls unter Einschränkungen, ermöglichen sollen und generellen Verbotsentscheidungen regelmäßig entgegenstehen. Setzt die Durchführungsverordnung zur Wirkstoff-Neugenehmigung - wie im Glyphosat-Fall - nicht selbst klare Vorgaben, sondern lässt stattdessen die Grundsätze zum Zuge kommen oder verweist sogar noch ausdrücklich auf sie, werden die vermeintlichen Sicherheitsanforderungen der Durchführungsverordnung noch einmal deutlich abgeschwächt, ohne dass man ihnen das ansieht.

\section{Fortlaufende Kontrolle und Handlungsoptionen}

Im Folgenden soll geklärt werden, welche Handlungsmöglichkeiten bestehen, wenn sich z.B. in Zukunft neue Erkenntnisse zu möglichen Risiken ergeben. ${ }^{314}$ Ein großes

313 S.o. C I.3.b).

314 Produkthaftrechtliche Fragen werden hier nicht behandelt. Siehe dazu etwa Schmitt, StoffR 2018, S. $241-244$. 
Problem besteht allerdings darin, dass kein Monitoring-System existiert, das die Langzeiteffekte der auf den Markt gebrachten Pestizide überwachen würde. ${ }^{315}$

\section{Europäische Ebene}

\section{1. Überprüfung der Wirkstoffgenehmigung}

Gemäß Art. 21 VO (EG) 1107/2009 kann die Kommission die Genehmigung eines Wirkstoffes jederzeit überprüfen und eine Aufhebungs- oder Änderungsverordnung erlassen, wenn insbesondere die Genehmigungskriterien des Art. 4 VO (EG) 1107/2009316 nicht mehr erfüllt sind. Das dürfte auch den Fall erfassen, dass im Nachhinein geklärt wird, dass sie schon von Anfang an nicht erfüllt waren, eine (Neu-)Genehmigung also gar nicht hätte erteilt werden dürfen. Vorhandene Bestände von Pflanzenschutzmitteln, die einen nicht mehr zugelassenen Wirkstoff enthalten, dürfen grundsätzlich noch für einen Übergangszeitraum von maximal sechs Monaten („Abverkaufsfrist“) weiter genutzt werden und müssen spätestens nach einem weiteren Jahr erst restlos entsorgt sein. Der befristete Abverkauf findet aber nicht statt, wenn die Genehmigung aus Gründen des Gesundheits- oder Umweltschutzes aufgehoben wurde. ${ }^{317}$

\section{2. Überprüfung der Pflanzenschutzmittel-Zulassung}

Art. 44 VO (EG) 1107/2009 regelt die Möglichkeit der Mitgliedstaaten, eine Pflanzenschutzmittel-Zulassung nachträglich zu beschränken oder aufzuheben. Das betrifft insbesondere den Fall, dass „es Anzeichen dafür gibt“, dass die Sicherheitsanforderungen des zentralen Art. 29 VO (EG) 1107/2009, der wiederum auf Art. 4 Abs. 3 (u.a. Gesundheits- und Umweltschutz) verweist, nicht mehr erfüllt sind oder Zulassungsinhaber die auferlegten Bedingungen nicht erfüllen.

\section{Notfallmaßnahmen}

Art. 69 ff. VO (EG) 1107/2009 erlauben es der Kommission, im Zusammenwirken mit den Mitgliedstaaten Notfallmaßnahmen zu treffen. Sowohl der Wirkstoff als auch Pflanzenschutzmittel können unverzüglich aus dem Verkehr gezogen werden, wenn „davon auszugehen“ ist, dass sie „wahrscheinlich ein schwerwiegendes Risiko“ für Mensch, Tier oder Umwelt darstellen. Subsidiär darf auch ein Mitgliedstaat nach Art. 71 VO (EG) 1107/2009 vorläufige Schutzmaßnahmen ergreifen.

315 S. zu entsprechenden Forderungen des EP die EP-Resolution 16.01.2019, (Fn. 95), insbes. $\int \mathbb{S} 16$ f. sowie General Considerations (sub X).

316 S.o. C I.2.a) und b).

317 Art. 21 Abs. 3 Satz 2 i.V.m. Art. 20 Abs. 2 VO (EG) 1107/2009; \$28 Abs. 4 PflSchG. 


\section{Aktionsrahmen für die nachhaltige Verwendung von Pestiziden}

Art. 4 der Richtlinie 2009/128/EG über einen Aktionsrahmen der Gemeinschaft für die nachhaltige Verwendung von Pestiziden (Pestizid-Rahmenrichtlinie) ${ }^{318}$ verpflichtet die Mitgliedstaaten, Nationale Aktionspläne zur nachhaltigen Anwendung von Pflanzenschutzmitteln zu verabschieden. Schon aus der Verwendung des Begriffs der „Pestizide“ anstelle von „Pflanzenschutzmitteln“ lässt sich erkennen, dass diese Richtlinie nicht aus dem Bereich der Agrarwirtschaft, sondern des Umweltschutzes kommt. Die Aktionspläne sollen quantitative Vorgaben, Ziele, Maßnahmen und Zeitpläne zur Verringerung der Risiken und Auswirkungen der Anwendung von Pflanzenschutzmitteln auf die Gesundheit von Mensch und Tier sowie auf den Naturhaushalt umfassen. Es sollen ein „integrierter Pflanzenschutz“ entwickelt und alternative Konzepte zur Verringerung der Abhängigkeit von der Verwendung von Pestiziden gefördert werden (Erwägungsgrund 5). Dafür gibt die Richtlinie bestimmte Themen wie z.B. den Gewässerschutz oder die Information der Öffentlichkeit vor, die die einzelstaatlichen Aktionspläne im Sinne der Richtlinie aufgreifen und ausgestalten müssen. ${ }^{319}$ In Reaktion auf den Bericht der Kommission über die Umsetzung der Anforderungen der Richtlinie 2009/128/EG (Art. 4 Abs. 3, 16) in den Mitgliedstaaten, hat die Berichterstatterin des Europäischen Parlaments Anfang 2019 ihre tiefe Besorgnis über die schlechte Umsetzung ("deep concern over the poor implementation") zum Ausdruck gebracht und die Kommission aufgerufen, ihrer Rolle als Hüterin des Unionsrechts gegen „evidente Nicht-Befolgung“ seitens etlicher Mitgliedstaaten nachzukommen. ${ }^{320}$ Im Verhältnis zwischen der Pflanzenschutzmittelverordnung (EG) 1107/2009 und der Richtlinie 2009/128/EG, fehlt es also, obwohl beide aufeinander bezogen sind, an Konsistenz.

Eine wesentliche Ergänzung der Pestizid-Rahmenrichtlinie stellt die Verordnung (EG) 1185/2009 über Statistiken zu Pestiziden ${ }^{321}$ dar. Wie ihr Erwägungsgrund 1 verdeutlicht, dient sie der Umsetzung von Umweltprogrammen der EG/EU, insbesondere aber dem Ziel, die Auswirkungen von Pestiziden - insbesondere solchen, die in der Landwirtschaft verwendet werden - auf die menschliche Gesundheit und die Umwelt weiter zu verringern. Die Notwendigkeit einer „nachhaltigeren Verwendung von Pestiziden“ soll sich in einer „mit dem erforderlichen Schutz von Kulturpflanzen zu vereinbarende[n] deutliche[n] Verringerung der Risiken und der Verwendung von Pestiziden“ niederschlagen. Die Verordnung (EG) 1185/2009 schafft deshalb einen gemeinsamen Rahmen für die systematische Erstellung europäischer Statistiken über das Inverkehrbringen und die Verwendung von Pestiziden. Erfasst werden die jähr-

318 Richtlinie 2009/128/EG des Europäischen Parlaments und des Rates vom 21. Oktober 2009 über einen Aktionsrahmen der Gemeinschaft für die nachhaltige Verwendung von Pestiziden, ABl. (EG) L 309 v. 24.11.2009, S. 71.

319 Dazu noch u. D II.2.

320 EP, Report on the implementation of Directive 2009/128/EC on the sustainable use of pesticides vom 30.01.2019, 2017/2284(INI), A8-0045/2019, S. 4, 6. S. auch EP-Resolution vom 16.01.2019, (Fn. 95), $\$ \$ 10 \mathrm{f}$.

321 Verordnung (EG) 1185/2009 des Europäischen Parlaments und des Rates vom 25. November 2009 über Statistiken zu Pestiziden, ABl. (EU) L 324 v. 10.12.2009, S. 1. 
lichen Mengen an Pestiziden, die in Verkehr gebracht und die Mengen, die verwendet werden. Gemäß Anhang II, Abschnitt 1 der Verordnung erfassen die Statistiken die in Anhang III aufgeführten Stoffe, die in Pestiziden enthalten sind, die in den einzelnen Mitgliedstaaten für jede einzelne ausgewählte Kulturpflanze verwendet werden. Die Auswahl trifft der einzelne Mitgliedstaat, der dabei aber diejenigen Kulturpflanzen berücksichtigen muss, die die größte Relevanz für die nationalen Aktionspläne gemäß Art. 4 der Richtlinie 2009/128/EG haben. Sowohl Glyphosat als auch Glufosinat sind in der Chemikalienklasse der „Organophosphor-Herbizide“ (H7.14) in Anhang III der Verordnung (EG) 1185/2009 als Wirkstoffe erfasst, die somit statistisch bearbeitet werden müssen.

Grundsätzlich übermitteln die Mitgliedstaaten genaue Daten an die Kommission, die sie auch der Öffentlichkeit zur Verfügung stellt. Allerdings bestimmt Art. 3 Abs. 4 VO 1185/2009 hierzu einschränkend:

„Aus Gründen der Vertraulichkeit aggregiert die Kommission (Eurostat) die Daten vor ihrer Veröffentlichung nach den chemischen Produktklassen oder -kategorien gemäß Anhang III und berücksichtigt dabei in gebührender Weise den Schutz vertraulicher Daten auf der Ebene der einzelnen Mitgliedstaaten. Nach Artikel 20 der Verordnung (EG) Nr. 223/2009 werden vertrauliche Daten von den einzelstaatlichen Stellen und der Kommission (Eurostat) ausschließlich für statistische Zwecke verwendet.“

Danach darf die Öffentlichkeit also nicht erfahren, wieviel Glyphosat in den einzelnen Mitgliedstaaten in den Verkehr gebracht und für die dort repräsentativen Kulturpflanzen verwendet wird. Denn nach außen darf nur von „Organophosphor-Herbiziden" die Rede sein. Selbst wer die chemischen Kenntnisse besitzt, um dahinter das Richtige zu vermuten, kann nicht erkennen, welche Mengen auf Glyphosat und welche auf Glufosinat entfallen. Dem entspricht, wie schon gesehen, auch die deutsche Publikationspraxis. ${ }^{322}$ Inwieweit eine solche Praxis allerdings aus Gründen des Schutzes vertraulicher Daten geboten sein soll und warum die Zusammenfassung in Chemikalienklassen schematisch vorgegeben wird, statt in Art. 3 Abs. 4 VO 1185/2009 die Notwendigkeit der Vertraulichkeit zu verlangen, bleibt unerfindlich. Das Ergebnis ist ein hohes Maß an Intransparenz, das eine wirksame Kontrolle durch Presse und Öffentlichkeit unverhältnismäßig erschwert.

\section{Mitgliedstaatliches Recht (Deutschland)}

\section{Widerruf und Rücknahme von Pflanzenschutzmittel-Zulassungen}

Nach $₫ 39$ Abs. 1 PfLSchG ist eine Zulassung unbeschadet des $₫ 49$ VwVfG zu widerrufen, wenn die Voraussetzungen des Art. 44 Abs. 3 lit. a, c oder e VO (EG) 1107/2009 vorliegen, also z.B. Anforderungen des Art. 29 VO [EG] 1107/2009 nicht 
mehr erfüllt sind ${ }^{323}$ oder der Zulassungsinhaber wiederholt gegen seine Pflichten aus Art. 56 VO (EG) 1107/2009 (Meldepflichten betreffend neue Informationen zu schädlichen Auswirkungen) verstoßen hat. Sie kann gemäß $\$ 39$ Abs. 2 PflSchG widerrufen werden, wenn es bloße Anzeichen dafür gibt, dass die Anforderungen des Art. $29 \mathrm{VO}$ (EG) 1107/2009 nicht mehr erfüllt sind, oder wiederholt die Zusammensetzung des in Verkehr gebrachten Pflanzenschutzmittels wesentlich von der Zusammensetzung des zugelassenen Pflanzenschutzmittels abweicht. Gesehen werden muss aber, dass die deutsche Behörde den Widerruf nur innerhalb eines Jahres nach Kenntnis der zugrunde liegenden Tatsachen vornehmen kann. ${ }^{324}$ Agiert sie insoweit nachlässig, könnte dies die Verpflichtung Deutschlands aus Art. 44 Abs. 3 VO (EG) 1107/2009 vereiteln, wonach der Mitgliedstaat die Zulassung in den dort genannten Fällen aufheben muss. In einem solchen Fall würde sich die Frage stellen, ob der Rechtsgedanke, der in der Ein-Jahresfrist zum Ausdruck kommt, auch Teil des europäischen Rechtsstaatsprinzips ist. Dadurch können sich die Mitgliedstaaten allerdings nicht ihrer europarechtlichen Pflicht entziehen.

Zulassungen sind gemäß $\ 39$ Abs. 3 PflSchG unter Beachtung des $\ 48$ VwVfG zurückzunebmen, wenn der Antragsteller die Zulassung durch arglistige Täuschung, Drohung oder Bestechung oder durch falsche oder irreführende Angaben erlangt hat. In diesen Fällen war die Zulassung von Anfang an rechtswidrig. Deshalb ist die Rücknahme grundsätzlich einfacher zu bewirken als der Widerruf von Zulassungen, die ursprünglich rechtmäßig waren, bis sich die Lage verändert hat. Dem entsprechend besteht auch keine zeitliche Begrenzung für die Rücknahme der Zulassung von Pflanzenschutzmitteln, soweit jedenfalls arglistige Täuschung, Drohung oder Bestechung involviert waren. ${ }^{325}$ Soweit es die praxisrelevanten falschen oder irreführenden Angaben betrifft, besteht aber auch hier die Gefahr, dass Behörden - aus welchen Gründen auch immer - untätig bleiben und es so ermöglichen könnten, dass eine Zulassung aufgrund falscher Angaben jedenfalls nicht aufgrund $\$ 39$ Abs. 3 PflSchG zurückgenommen werden dürfte. Wenn in solchen Fällen allerdings zugleich die Erfüllung der Sicherheitsanforderungen des Art. 29 VO (EG) 1107/2009 infrage gestellt wird, muss bzw. kann die Zulassung aufgrund $\$ 39$ Abs. 1, 2 PflSchG widerrufen werden.

In der öffentlichen Diskussion wird für den Fall eines Glyphosat-Ausstiegs gelegentlich auf horrende Entschädigungsforderungen der betroffenen Unternehmen verwiesen. Entschädigung in Geld wird von vornherein nur gewährt, wenn Betroffene oder ihre Rechtsvorgänger nicht durch Zuwiderhandlungen selbst Anlass für die Maßnahme gegeben haben ( $\$ 54$ Abs. 3 PflSchG). Diesem Rechtsgedanken entsprechen auch die Entschädigungsregeln der $\iint 48 \mathrm{f}$. VwVfG. Entschädigung kommt daher nur in Fällen der Aufhebung von Zulassungen in Betracht, wenn z.B. aufgrund neuer wissenschaftlicher Erkenntnisse klar wird, dass die Anforderungen des Art. 29 VO (EG) 1107/2009 von Anfang an nicht erfüllt waren oder nicht mehr erfüllt sind. Die Entschädigung ist aber selbst dann nur zu gewähren, „soweit dies zur Abwendung

323 D.h., Anforderungen aus $₫ 29$ sind nicht mehr erfüllt, eine Bedingung für die Zulassung oder Verpflichtungen aus der VO wurden nicht erfüllt. S. D I.2.

$324 \$ 39$ Abs. 1 PflSchG i.V.m. $\$ \int 49$ Abs. 3 Satz 2, 48 Abs. 4 VwVfG.

325 Siehe die Gegenausnahme in $\$ 48$ Abs. 4 VwVfG sowie Fn. 324. 
oder zum Ausgleich unbilliger Härten geboten erscheint“. ( $\$ 54$ Abs. 2 PflSchG). Es erscheint gerade nicht als unbillige Härte, wenn Unternehmen, insbesondere solche, die an der "Glyphosate Task Force“ beteiligt waren, nach monatelangen Kämpfen um die Bewertung des Risikos keine Entschädigung erhalten. Denn sie mussten von vornherein damit rechnen, dass sich die Risiken im Zusammenhang mit diesem Wirkstoff und seinen Anwendungsprodukten im Laufe der Zeit erhärten oder besser nachgewiesen werden könnten. Von der Möglichkeit relativ schnellen Wandels ging auch die Europäische Kommission aus, als sie die Befristung der Durchführungsverordnung zur erneuten Glyphosat-Genehmigung auf fünf Jahre genau damit begründete. ${ }^{326}$

\section{Nationaler Aktionsplan zur nachhaltigen Anwendung von Pestiziden}

Gemäß dem Auftrag aus Art. 4 der Pestizid-Rahmenrichtlinie 2009/128/EG 327 bestimmt $₫ 4$ PflSchG, dass in Deutschland ein Nationaler Aktionsplan (NAP) zur nachhaltigen Anwendung von Pestiziden erstellt wird, und zwar unter Mitwirkung von Bund und Ländern, Verbänden und Öffentlichkeit. Der aktuelle deutsche Plan wurde am 10. April 2013 von der Bundesregierung beschlossen. ${ }^{328}$ Der Einfluss des Bundeslandwirtschaftsministeriums (BMEL) lässt sich schon an der Bezeichnung „Nationaler Aktionsplan zur nachhaltigen Anwendung von Pflanzenschutzmitteln“ ablesen, da sie den Begriff „Pestizide“ aus der Rahmenrichtlinie durch „Pflanzenschutzmittel“" ersetzt.

Um den einzelnen Vorgaben aus der Richtlinie Rechnung zu tragen, wurde das Pflanzenschutzgesetz nachgebessert, insbesondere in den Bereichen Aus- und Fortbildung $(\mathbb{9}$ ), Regulierung der Anwendung durch Festlegung von Anwendungsgebieten und -bedingungen ( $(12)$, Auflagen für den Verkauf (\$23), Pflanzenschutzgeräte (\$16), Einsatz von Flugzeugen $(\mathbb{1} 18)$, Trinkwasser- und Gewässerschutz $(\mathbb{S} 12$, 22), Verringerung des Einsatzes von Pflanzenschutzmitteln in bestimmten Gebieten ( $\mathbb{S} 12,17,22 \mathrm{PflSchG)}$ sowie integrierter Pflanzenschutz nach „guter fachlicher Praxis“ ( $\$ 3$ PflSchG). Bezüglich der Pflicht zur Information der Öffentlichkeit über Nutzen und Risiken, verweist das BMEL auf Internetangebote. Die Vorgaben der Richtlinie zur Handhabung, Lagerung und Verpackung von Pflanzenschutzmitteln sowie der Umgang mit Restmengen verteilen sich über das Chemikalien- und Gefahrstoff-, Wasserhaushalts-, Immissionsschutz- und Gütertransportrecht. ${ }^{329}$ Außer-

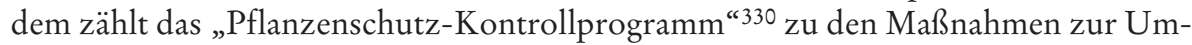
setzung der Pestizid-Rahmenrichtlinie. Ob der Nationale Aktionsplan den europäischen Anforderungen gerecht wird, soll hier nur anhand einer zentralen Vorgabe exemplarisch betrachtet werden, nämlich der Verpflichtung zur Verringerung des Einsatzes von Pflanzenschutzmitteln in bestimmten kritischen Gebieten.

326 S.o. C I.3.b).

327 S.o. D I.4.

328 BMEL, Bekanntmachung des Nationalen Aktionsplans zur nachhaltigen Anwendung von Pflanzenschutzmitteln vom 10.04.2013, Bundesanzeiger vom 15. Mai 2013.

329 BMEL, NAP, (Fn. 328), S. 11-13.

330 S.u. D II.3. 
Art. 12 der Pestizid-Rahmenrichtlinie 2009/128/EG verlangt Maßnahmen zur Verringerung des Einsatzes von Pflanzenschutzmitteln auf öffentlich zugänglichen Flächen, speziellen Schutzgebieten und kürzlich behandelten Flächen, die für landwirtschaftliche Arbeitskräfte zugänglich sind. Dabei sind geeignete Risikomanagementmaßnahmen zu treffen und ist „der Verwendung von Pflanzenschutzmitteln mit geringem Risiko im Sinne der Verordnung (EG) Nr. 1107/2009 sowie biologischen Bekämpfungsmaßnahmen ... der Vorzug zu geben“. Nach Auffassung des BMEL würden diese Anforderungen durch $\$ \int 12,17,22$ PflSchG erfüllt. Sieht man sich diese Vorschriften jedoch genauer an, ergeben sich Zweifel. $\$ 12$ Abs. 1 PflSchG verbietet zwar die Anwendung von Pflanzenschutzmitteln auf befestigten Freilandflächen, auf nicht-landwirtschaftlich genutzten Freilandflächen sowie „in oder unmittelbar an oberirdischen Gewässern und Küstengewässern“. Zugleich sieht die Vorschrift aber auch die Möglichkeit von Ausnahmen vor, „wenn der angestrebte Zweck vordringlich ist und mit zumutbarem Aufwand auf andere Art nicht erzielt werden kann und überwiegende öffentliche Interessen, insbesondere des Schutzes der Gesundheit von Mensch und Tier oder des Naturhaushaltes, nicht entgegenstehen“. Gemäß \17 PflSchG müssen Pflanzenschutzmittel, die auf öffentlich zugänglichen Flächen angewandt werden sollen, ein spezielles Zulassungsverfahren durchlaufen. Die Genehmigung wird aber erteilt, wenn erstens an der Anwendung ein öffentliches Interesse besteht und zweitens eine Prüfung ergibt, dass das Pflanzenschutzmittel aufgrund seiner chemischen Eigenschaften bei bestimmungsgemäßer und sachgerechter Anwendung keine schädlichen Auswirkungen auf die Allgemeinheit hat. $\mathbb{S} 22 \mathrm{PflSchG} \mathrm{räumt} \mathrm{schließlich} \mathrm{den} \mathrm{Bundesländern} \mathrm{die} \mathrm{Befugnis} \mathrm{ein,} \mathrm{ein-}$ schränkende Regelungen zur Anwendung von Pflanzenschutzmitteln in Schutzgebieten zu treffen. Ohne dass hier eine abschließende Begutachtung gerade auch im Hinblick auf die Bundesländer erfolgen kann, erscheint fraglich, ob die genannten Vorschriften dem grundlegenden Ziel des Art. 12 der Pestizid-Rahmenrichtlinie 2009/128/EG entsprechen, den Verbrauch auf bestimmten kritischen Flächen zu reduzieren. Zum einen dürften die Voraussetzungen der Ausnahmeklauseln (Schutz der Gesundheit und des Naturhaushalts bzw. Fehlen schädlicher Auswirkungen auf die Allgemeinheit) relativ leicht erfüllbar sein, da grundlegende Gefährlichkeitsaspekte schon Gegenstand der Bewertungen für die Zulassung eines Mittels gewesen sind. Vor allem aber ist den Vorschriften nicht zu entnehmen, wodurch der Verbrauch von Pestiziden wie z.B. glyphosathaltiger Mittel auf den kritischen Flächen abgesenkt werden könnte. Auch den grundsätzlichen Vorzug zugunsten von Pflanzenschutzmitteln mit geringem Risiko und biologischen Bekämpfungsmaßnahmen sucht man in den deutschen Vorschriften vergebens.

Datenerhebung ist ebenfalls Teil des Nationalen Aktionsplans. Wie schon gezeigt, regelt die Pestizidstatistik-Verordnung (EG) 1185/2009 die Art und Weise, wie die Mitgliedstaaten an der systematischen Erstellung von EU-Statistiken mitwirken. ${ }^{331}$ Danach müssen gerade auch für Glyphosat die jährlichen Mengen erfasst werden, die in Verkehr gebracht und verwendet werden. Objekt der Erhebung sind diejenigen 
Kulturpflanzen, die die größte Relevanz für die nationalen Aktionspläne haben. In Deutschland sind dies Winterweizen, Wintergerste, Winterroggen, Mais, Kartoffeln, Zuckerrüben, Tafelapfel, Hopfen und Wein. ${ }^{332}$ Das insoweit zuständige Julius-KühnInstitut ( $\$ 21 \mathrm{PflSchG)}$ veröffentlicht zwar im Rahmen der jährlichen „PAPA-Erhebungen“ ein „Wirkstoffranking" in Bezug auf diese Pflanzen. Die zugehörigen Wirkstoffmengenangaben beschränken sich aber auf Schätzwerte für Gesamtdeutschland, die das JKI selbst aufgrund einer nur „geringen Stichprobengröße“ für unsicher hält. ${ }^{333}$ Somit kann die Öffentlichkeit nicht erfahren, in welchen Regionen Deutschlands welche relevanten Kulturpflanzen besonders stark oder wenig mit Glyphosat behandelt sind, um ihre Kaufentscheidungen in einem zunehmend regional auftretenden Lebensmittelmarkt u.a. auch danach auszurichten. Zwar könnte die volle Transparenz dazu führen, dass einzelne land wirtschaftliche Betriebe z.B. wegen hoher regionaler Glyphosatmengen gemieden würden, obwohl sie selbst keine glyphosathaltigen Mittel verwenden. Dieses Problem wäre aber lösbar, indem das Produktlabel entsprechend informiert. Es leuchtet deshalb nicht ein, warum der Öffentlichkeit lediglich Durchschnittswerte für Gesamtdeutschland auf explizit unzureichender Datengrundlage präsentiert werden, obwohl genauere Daten auch aus den einzelnen Bundesländern vorhanden sind.

\section{Verkehrs- und Anwendungskontrollen, insbesondere das „Pflanzenschutz- Kontrollprogramm“}

In Deutschland sind die Bundesländer für alle wesentlichen Aufgaben im Bereich der Verkehrs- und Anwendungskontrolle bei Pflanzenschutzmitteln zuständig. ${ }^{334}$ Damit ergeben sich unvermeidlich Unterschiede im Vollzug der Überwachung. Die Vollzugsdichte in den einzelnen Bundesländern schwankt mit Blick auf unterschiedliche politische Positionen in den Landesregierungen, unterschiedliche Personal- und Sachausstattung bei den Landesbehörden, aber auch mit Blick auf die unterschiedliche Bedeutung, die die Agrarwirtschaft in den einzelnen Ländern hat. Um die Handhabung der Kontrollaufgaben dennoch in ganz Deutschland zu harmonisieren, haben Bund und Länder ein gemeinsames „Pflanzenschutz-Kontrollprogramm“ installiert, das gemeinsame Kontrollstandards entwickelt und Ergebnisse und Vorschläge zur Überwachung der Pflanzenschutzmittelverwendung in Form von Jahresberichten veröffentlicht. ${ }^{335}$

Ob solche Koordinierungsmaßnahmen den Gesetzesvollzug verbessern, erscheint allerdings zweifelhaft. Sieht man z.B. in den letzten Jahresbericht von 2017 hinein, wird dort u.a. mitgeteilt, es habe sich bei den Kontrollen von Händlern „wie in den vergangenen Jahren“ gezeigt, „dass bei rund einem Drittel der Betriebe mindestens ein Pflanzenschutzmittel angeboten wurde, das nicht mehr verkauft werden durfte",

332 Siehe PAPA (Panel Pflanzenschutzmittel-Anwendungen) - Seite des Julius-Kühn-Instituts, abrufbar unter: https://papa.julius-kuehn.de/ (15.06.2019).

333 S.o. A II.1.

334 S.o. B II.2.

335 S.o. B II.2. 
wobei die Beanstandungsquote von 27,4\% (2016) auf 32,3\% (2017) gestiegen sei. ${ }^{336}$ Als Grund für diese Erhöhung wird eine „verstärkt[e] Überprüfung von Onlineangeboten“ genannt, wo sich häufig unzureichende Informationen befänden. ${ }^{337} \mathrm{Weitere}$ Maßnahmen wurden insoweit nicht vorgeschlagen. Soweit es die Einhaltung von Vorschriften zur Vermeidung von Pflanzenschutzmitteleinträgen durch Abdrift in Gewässer betrifft, sind die Ausführungen des Berichts besonders interessant, weil sie gerade bei glyphosathaltigen Pflanzenschutzmitteln unbedingt zu vermeiden ist ${ }^{338}$ und in diesem Bereich ein Schwerpunkt der bundesweiten Kontrollen im Zeitraum bis 2017 lag. Wie die Überwachung für 2017 jedoch zeigte, hatten die Landwirte auf 89 von 399 kontrollierten Schlägen (bewirtschafteten Flurstücken) keine ausreichenden Maßnahmen zum Gewässerschutz getroffen, wobei die Beanstandungsquote „von 2014 bis 2017 kontinuierlich angestiegen“ sei. ${ }^{339}$ Angesichts dieses Befundes gelangt die AG Pflanzenschutzmittelkontrolle nicht etwa zu dem Schluss, dass die Rechtsdurchsetzung forciert werden müsse, sondern zieht eine „geänderte Kontrollmethode“ sowie die „Fokussierung der Kontrollen auf problematische Betriebe“ als mögliche Erklärungen für die steigende Beanstandungsquote in Betracht. Deshalb sei auch eine Übertragung auf die Gesamtheit der landwirtschaftlichen Betriebe „nicht möglich“. Letztlich seien die Anwender nicht ausreichend informiert, weshalb man die Informationsbroschüren verbessert und einen neuen Flyer zur Abdriftminderung aufgelegt habe. Abschließend weist der Jahresbericht darauf hin, dass die Beendigung des Schwerpunkts Gewässerschutz nicht bedeute, dass Kontrollen in diesem Bereich in Zukunft unterblieben.

Die Beispiele zeigen einerseits, wie hoch die Kontrolldichte ist, wie viele Betriebe also tatsächlich kontrolliert wurden, und wie hoch die Beanstandungsquote in den einzelnen Bereichen ist. Vorausgesetzt, dass alle Daten korrekt sind, erhöht diese Art der transparenten Erfolgskontrolle sicherlich den Schutz gegen Kontrolldefizite, wie sie durch Personalabbau, politischen Einfluss oder mangelnde Einsatzbereitschaft entstehen können. Andererseits vermittelt der Jahresbericht nicht den Eindruck, man habe wirklich wissen wollen, warum Beanstandungsquoten ungeachtet des aufwändigen „Pflanzenschutz-Kontrollprogramms“ gestiegen und nicht gesunken sind. Die allzu schnell gegebenen pauschalen Erklärungen für festgestellte Abweichungen stellen sich als Rechtfertigungsversuche dafür dar, es beim Status quo (d.h. Kontrolle in unverändertem Ausmaß mit gelegentlichen Kontrollschwerpunkten) zu belassen. An dieser Stelle erweist es sich als Problem, dass auch auf europäischer Ebene kein Mechanismus besteht, mit dem die effektive Umsetzung von risikomindernden Vorgaben aus einer Wirkstoffgenehmigung in den Mitgliedstaaten kontrolliert würde. ${ }^{340}$

$336 B V L$, (Fn. 102), S. 1, 17 (Kontrolliert wurden ca. 2.100 Betriebe).

337 Ibid. S. 20.

338 Zur toxischen Wirkung von Glyphosat auf Wasserorganismen A II.2.

339 Auch zum Folgenden BVL, (Fn. 102), S. 2, 23 f.

340 Hierzu auch die kritischen Anmerkungen der Europäischen Ombudsfrau im Fall 12/2013/ MDC, (Fn. 208), $\$ \$ \int 41 \mathrm{ff}$. 


\section{E. Die Neugenehmigung von Glyphosat im Lichte übergeordneter Maßstäbe und widerstreitender Individualrechte}

\section{Vorsorgeprinzip}

\section{Inhalt und Anforderungen}

Gemäß Art. 1 Abs. 4 Satz 1 VO (EG) 1107/2009 „beruhen“ alle Bestimmungen der europäischen Pflanzenschutzverordnung auf dem Vorsorgeprinzip. Das heißt, sie haben den Zweck sicherzustellen, dass in Verkehr gebrachte Wirkstoffe oder Pflanzenschutzmittel weder die Gesundheit von Mensch und Tier noch die Umwelt beeinträchtigen. Im Lichte dieser Zielvorgabe sind sie auszulegen und anzuwenden. Zugleich ist das Vorsorgeprinzip als primärrechtlicher Grundsatz des europäischen Umweltrechts in Art. 191 Abs. 2 UAbs. 1 Satz 2 AEUV gemeinsam mit dem Vorbeugeprinzip verankert. Der EuGH hat es im Zusammenhang mit der Agrarpolitik schon in den 90 -er Jahren anerkannt. ${ }^{341}$

Während das Vorbeugeprinzip sich darauf richtet, Gefahren für die Umwelt präventiv im Rahmen der Verhältnismäßigkeit auszuschließen, bezweckt das Vorsorgeprinzip nach heutigem Verständnis, Risiken für die Umwelt schon im Vorfeld zu vermeiden, auch wenn noch keine Gefahr im polizeilichen Sinne vorliegt. ${ }^{342}$ Entscheidend für das Eingreifen des Vorsorgeprinzips ist das Vorliegen eines „Risikos“, wofür die bloße Möglichkeit genügt, dass es zu einer "nachteiligen Wirkung“ für ein geschütztes Rechtsgut kommt, ohne dass eine kausale Beziehung nachgewiesen werden muss. ${ }^{343}$ Als geschützte Rechtsgüter kommen insbesondere die in Art. 1 Abs. 3 Verordnung (EG) 1107/2009 genannten Rechtsgüter, also die Gewährleistung eines „hohen Schutzniveaus für die Gesundheit von Mensch und Tier und für die Umwelt" in Betracht.

Das Vorsorgeprinzip verlangt von den zuständigen Behörden eine objektive und wissenschaftlich einwandfreie Risikobewertung, ein Risikomanagement unter Einbeziehung aller Betroffenen und eine transparente Risikokommunikation. ${ }^{344} \mathrm{Um}$ ein Risiko angemessen zu bewerten, müssen die Wahrscheinlichkeit und das Ausmaß des potentiellen Schadens in Beziehung zueinander gesetzt werden. Obwohl die Schwelle zum Eingreifen grundsätzlich niedrig ist, steht das Vorsorgeprinzip im Kontext der gesamten europäischen Rechtsordnung, so dass es letztlich zur Abwägung mit anderen Interessen und Rechtsgütern anhand des Maßstabs der Verhältnismäßigkeit kommt. Konkret muss in einem ersten Schritt mithilfe wissenschaftlicher Methoden das Risiko ermittelt und bewertet werden, in einem zweiten Schritt muss bewertet

341 EuGH, Rs. C-180/96, Vereinigtes Königreich v. Kommission [BSE], ECLI:EU:C:1998:192, Rn. 98 ff.; EuGH, Rs. C-157/96, The Queen v. Ministry of Agriculture, Fisheries and Food, Commissioners of Customs \& Excise, ex parte National Farmers' Union and Others, ECLI:EU:C:1998:191, Rn. 63.

342 Heselhaus, in: Pechstein/Nowak/Häde (Hrsg.), Art. 191 AEUV, Rn. 62.

343 Ibid., Rn. 64 m.w.N.

344 Vgl. Kommission, Mitteilung 2000/1 vom 02.02.2000 über die Anwendbarkeit des Vorsorgeprinzips. 
werden, welcher Risikograd als nicht mehr hinnehmbar gilt. ${ }^{345}$ Zwar wird vertreten, dass „sozial-adäquate Restrisiken“ hinzunehmen seien. ${ }^{346}$ Ein begrenztes Risiko mit schwerer möglicher Schadensfolge (z.B. Krebserkrankung) ist aber niemals sozialadäquat. Soweit die Pflanzenschutzmittelverordnung nur „unvertretbare“ oder „unannehmbare" Umweltauswirkungen ausschließt (Art. 4 Abs. 3 VO [EG] 1107/2009), bezieht sich dies jedenfalls nicht auf den Wahrscheinlichkeitsgrad des Risikos, sondern nur auf das Schadensfolgenlevel, das noch hingenommen wird.

Im Hinblick auf mögliche Handlungs- und Schutzverpflichtungen aus dem Vorsorgeprinzip gilt: Einerseits muss nicht abgewartet werden, bis der Schaden eintritt; ${ }^{347}$ andererseits genügen rein hypothetische Befürchtungen nicht, um Eingriffe in Grundfreiheiten und Grundrechte vorzunehmen. Es müssen also ein berechtigter Grund bzw. Indizien für die Annahme eines Risikos bestehen. Dabei folgt aus dem Verhältnismäßigkeitsprinzip, dass Maßnahmen zur Minderung des Risikos vorrangig in Betracht gezogen werden müssen, während Verbote (z.B. von Wirkstoffen) das letzte Mittel sind. Es trifft aber nicht zu, dass das Vorsorgeprinzip in einem Szenario der festgestellten Ungewissheit nur angewendet werden „könnte“ oder „sollte“.348 Vielmehr muss es als bindender Rechtsgrundsatz des Primärrechts angewendet werden und besteht allenfalls auf der Rechtsfolgenseite Spielraum.

Spezielle Ausformungen des Vorsorgeprinzips finden sich im Sekundärrecht, wobei zwei Regelungen gerade hier von besonderer Bedeutung sind. Zum einen erlaubt Art. 7 Lebensmittelrecht-Verordnung (EG) 178/2002 ${ }^{349}$ vorsorgliche Maßnahmen im Interesse der Lebensmittelsicherheit. Danach können „in bestimmten Fällen, in denen nach einer Auswertung der verfügbaren Informationen die Möglichkeit gesundheitsschädlicher Auswirkungen festgestellt wird, wissenschaftlich aber noch Unsicherheit besteht, ... vorläufige Risikomanagementmaßnahmen ${ }^{350}$ zur Sicherstellung des in der Gemeinschaft gewählten hohen Gesundheitsschutzniveaus getroffen werden, bis weitere wissenschaftliche Informationen für eine umfassendere Risikobewertung vorliegen“. Die Maßnahmen müssen verhältnismäßig sein und in angemessenen Zeitabständen überprüft werden. Zum anderen stellt Art. 1 Abs. 4 Satz 2 VO (EG) 1107/2009 es den Mitgliedstaaten frei, das Vorsorgeprinzip anzuwenden, wenn wissenschaftliche Ungewissheit besteht, ob die in ihrem Hoheitsgebiet zuzulassenden Pflanzenschutzmittel Gefahren für die Gesundheit von Mensch oder Tier oder die Umwelt bergen. Wie gezeigt, kann diese Norm zwar nicht als eigenständige Ermächtigungsgrundlage

345 EuG, Rs. T 13/99, Pfizer, ECLI:EU:T:2002:209, Rn. 145.

346 So Heselhaus, in: Pechstein/Nowak/Häde (Hrsg.), Art. 191 AEUV, Rn. 64, unter Bezugnahme auf GA Mischo im Fall Greenpeace France (m.N.).

347 EuGH, Rs. C-157/96, The Queen v Ministry of Agriculture, Fisheries and Food, Commissioners of Customs E Excise, ex parte National Farmers' Union and Others, ECLI:EU:C: 1998:191, Rn. 63.

348 Hensel, ZLR 2016/4, S. 449.

349 Verordnung (EG) Nr. 178/2002 des Europäischen Parlaments und des Rates vom 28.01.2002 zur Festlegung der allgemeinen Grundsätze und Anforderungen des Lebensmittelrechts, zur Errichtung der Europäischen Behörde für Lebensmittelsicherheit und zur Festlegung von Verfahren zur Lebensmittelsicherheit, ABl. (EG) L 31 v. 01.02.2002, S. 1.

350 Siehe dazu die Legaldefinition in Art. 3 Nr. 12 VO (EG) Nr. 178/2002. 
für ein Verbot oder die Einschränkung von Pflanzenschutzmitteln herangezogen werden. Sie erweitert aber den Beurteilungs- und damit auch letztlich den Handlungsspielraum der Mitgliedstaaten, in einem Kontext der Ungewissheit unmittelbar auf Gefahr zu reagieren. ${ }^{351}$ Art. 2 Abs. 3 der Richtlinie 2009/128/EG ${ }^{352}$ über die nachhaltige Pestizidverwendung stellt klar, dass diese Richtlinie die Mitgliedstaaten nicht daran hindert, „bei der Einschränkung oder dem Verbot der Verwendung von Pestiziden unter bestimmten Bedingungen oder in bestimmten Gebieten das Vorsorgeprinzip anzuwenden.“

\section{Beachtung des Vorsorgeprinzips bei der Erneuerung der Glyphosatgenehmigung?}

Im Falle der Erneuerung der Glyphosat-Genehmigung gibt es unterschiedliche Positionen zur Frage, ob das Vorsorgeprinzip beachtet wurde. Das Europäische Parlament z.B. ,ist der Auffassung, dass mit dem Entwurf einer Durchführungsverordnung der Kommission kein hohes Schutzniveau für die Gesundheit von Mensch und Tier und für die Umwelt gewährleistet wird, das Vorsorgeprinzip nicht befolgt wird und die Durchführungsbefugnisse gemäß der Verordnung (EG) Nr. 1107/2009 überschritten werden “. ${ }^{353}$ Auf der Gegenseite wird wiederum eine Emotionalisierung der öffentlichen Diskussion beklagt, die nicht zwischen Wissen und „Metaphysik“ unterscheiden könne. Wer ein „Null-Risiko“ fordere, verlange einen „Gottesbeweis“. Es gehe aber nicht um ein Null-Risiko, sondern nur um das „akzeptable Risiko“, das der Gesetzgeber in Anlehnung an das festlege, was die Gesellschaft für ein akzeptables Restrisiko halte. Dafür entscheidend sei die Ermittlung der Menge, die ein Mensch aufnehmen könne, ohne dass gesundheitliche Beeinträchtigungen zu erwarten seien. ${ }^{354}$ Die Generalanwältin Sharpston stellte in der Rechtssache Blaise darauf ab, dass das vorlegende Gericht die Verordnung (EG) Nr. 1107/2009 insgesamt u.a. wegen Verletzung des Vorsorgeprinzips infrage gestellt habe. Nicht nur stelle die europäische Pflanzenschutzmittelverordnung mit ihrem System der vorherigen Genehmigungen selbst eine Vorsorgemaßnahme dar. Wegen der Komplexität des Rechtsgebiets bestehe auch ein weiter Gestaltungsspielraum für den Regelungsgeber. Da die Verordnung unter Berücksichtigung dieses Spielraums weder offensichtlich ungeeignet noch sonst offensichtlich fehlerhaft sei, stehe ihre Gültigkeit u.a. wegen des Vorsorgeprinzips nicht infrage. ${ }^{355}$ Obwohl die Ausführungen der Generalanwältin an entscheidenden Stellen

351 S.u. E I.

352 Fn. 318.

353 Ziffer 1 der Entschließung des Europäischen Parlaments vom 24. Oktober 2017, (Fn. 92). S.o. B I.3. m.N. Ebenso bereits Ziffer 1 der Entschließung des Europäischen Parlaments vom 13. April 2016, (Fn. 94). Kaus, StoffR 2016, S. 110, führt die Entschließung von 2016 als Argument dafür an, dass kein Grund bestehe, die erneute Genehmigung von Glyphosat unter Verweis auf das Vorsorgeprinzip zu versagen, verschweigt aber, dass dasselbe Parlament in Ziffer 1 die Verletzung dieses Prinzips feststellt.

354 Hensel, Zeitschrift für das gesamte Lebensmittelrecht, 2016/4, S. 447 f.

355 Schlussanträge der Generalanwältin Sharpston vom 12.03.2019, EuGH, Rs. C-616/17, Procureur de la République gegen Mathieu Blaise et al., ECLI:EU:C:2019:190. S.u. E III. 
zu pauschal bleiben, ist ihr darin beizupflichten, dass eine Verletzung des Vorsorgeprinzips eher die erneute Genehmigung von Glyphosat als die Verordnung selbst betrifft, zumal diese eine Orientierung aller Entscheidungen am Vorsorgeprinzip ausdrücklich verlangt (Art. 1 Abs. 4 Satz 1 VO [EG] 1107/2009). Eine Entscheidung des EuGH liegt noch nicht vor (Stand: 15.4.2019).

Es wurde bereits gezeigt, dass Glyphosat sowie Pflanzenschutzmittel, die diesen Wirkstoff enthalten, die Voraussetzungen des Art. 4 VO (EG) 1107/2009 erfüllen müssen. Danach sind z.B. in Bezug auf Umweltschäden nur „unannehmbare Auswirkungen" verboten, auf die Gesundheit von Menschen oder Tieren dürfen die Mittel aber „keine sofortigen oder verzögerten schädlichen Auswirkungen“ haben. ${ }^{356}$ Dieser Unterschied in der Formulierung muss sich auch in der Handhabung des Vorsorgeprinzips niederschlagen, auch wenn sie nicht den Wahrscheinlichkeitsgrad, sondern nur das Schadensausmaß betrifft. Wenn Gesundheitsschäden von vornherein nicht im Wege normativer Bewertung und Abwägung „,annehmbar“ sind, darf der Gesetzgeber ein womöglich akzeptables „Restrisiko“ hier auch nicht konsentieren. Allenfalls könnte argumentiert werden, der Schadenseintritt stelle sich als nahezu unwahrscheinliches Ereignis dar, weil eine Exposition von Menschen gegenüber dem schädlichen Mittel „unter realistisch anzunehmenden Verwendungsbedingungen vernachlässigbar“ ${ }^{\text {357 }}$ sei. Selbst wenn eine solche Ausnahme aber zulässig wäre, wären die genannten Voraussetzungen, wie Glyphosatfunde in Lebensmitteln und im menschlichen Körper zeigen, ${ }^{358}$ hier nicht erfüllt.

Das entscheidende Problem liegt im Glyphosat-Wiedergenehmigungsfall in der Tatsache, dass die Wissenschaft das Risiko unterschiedlich einschätzt, einzelne Studien unterschiedliche oder widersprüchliche Schlussfolgerungen liefern und damit auch die Bewertung umstritten ist. Es trifft zu, dass das Vorsorgeprinzip eine Antwort der Politik auf den Umgang mit Nichtwissen darstellt und erst das Ausmaß an Unsicherheit zu ermitteln ist, ${ }^{359}$ bevor das Vorsorgeprinzip zum Zuge kommen kann. Für Vorsorge genügt es aber nicht, auf eine herrschende Meinung zu verweisen, sondern muss auch Mindermeinungen Rechnung getragen werden, sofern sie nicht von vornherein abwegig sind. ${ }^{360}$ Die Mindermeinung, die ein Risiko erkennt, indiziert eine Schadensmöglichkeit, die durch vorsorgliche Maßnahmen angemessen minimiert oder auch ganz ausgeschlossen werden muss, je nach dem, wie schwer die möglichen Folgen sind und wie wahrscheinlich ihr Eintreten ist. Dabei ist das Verbot ultima ratio. Das EuG formulierte es im Fall Schweden/Kommission in Bezug auf den Pflanzenschutzmittel-Wirkstoff „Paraquat“ so:

356 S.o. C I.2.

357 So die Ausnahmeklauseln in Nr. 3.6 von Anlage II zur VO (EG) 1107/2009.

358 S.o. A II.2.

359 Eine andere Frage ist es, ob im Falle eines "fachwissenschaftlichen Erkenntnisvakuums" die plausible Einschätzung der Behörde zugrunde zu legen ist - zumindest soweit der Gesetzgeber im grundrechtsrelevanten Bereich die Maßstäbe hierfür festgelegt hat, oder ob die Gerichte diese Einschätzung mit Sachverständigenhilfe infrage stellen dürfen. Siehe zum deutschen Recht BVerfG, NJW 2019, 141 (143): keine Vermutung, dass die Gerichtsbarkeit über mehr Expertise als die Verwaltung verfügt.

360 Siehe Doubaire, ZUR 2017, S. 395 m.w.N. 
„Aus dieser Vorschrift [Art. 5 der früheren Richtlinie 91/414], ausgelegt in Verbindung mit dem Vorsorgegrundsatz, ergibt sich, dass, wenn es um die menschliche Gesundheit geht, das Vorliegen ernsthafter Anhaltspunkte, die, ohne die wissenschaftliche Ungewissheit zu beseitigen, vernünftige Zweifel an der Unbedenklichkeit eines Stoffes erlauben, der Aufnahme dieses Stoffes in Anhang I der Richtlinie 91/414 grundsätzlich entgegensteht. Denn der Vorsorgegrundsatz soll potenziellen Risiken vorbeugen. Dagegen können rein hypothetische Risiken, die auf nicht untermauerte wissenschaftliche Hypothesen gestützt sind, nicht berücksichtigt wer$\operatorname{den}(\ldots) .{ }^{“ 361}$

Soweit es das mögliche Gesundheitsrisiko (insbesondere Krebsrisiko) für Menschen und Tiere betrifft, liegen gewichtige Stellungnahmen und Anhaltspunkte vor, die ein solches Risiko bejahen:

- die Einstufung durch die IARC („wahrscheinlich krebserregend“),

- die Stellungnahmen von Christopher Portier und etlicher Kollegen und Kolleginnen aus allen relevanten Forschungsgebieten,

- statistische Studien, die einen Zusammenhang zwischen dem Glyphosatgebrauch und dem Auftreten des Non-Hodgkin-Lymphoms belegen,

- epidemiologische Studien, die ebenfalls einen Zusammenhang belegen, aber als nicht aussagekräftig zurückgewiesen wurden,

- Ergebnisse von Beweisaufnahmen in Gerichtsverfahren in den USA. ${ }^{362}$

Die hieran anknüpfenden Zweifel an der Unbedenklichkeit des Wirkstoffes Glyphosat versuchten die mit der Stoffbewertung befassten Behörden durch eine unterschiedliche Gewichtung der Beweiskraft der Daten ("weight of evidence approach") zu zerstreuen. ${ }^{363}$ Sie weisen zutreffend darauf hin, dass die Gewichtung der verfügbaren Nachweise von Faktoren wie der Qualität der Daten, der Einheitlichkeit der Ergebnisse, der Art und Schwere von Auswirkungen und der Relevanz der Informationen abhängt. Je mehr Informationen vorliegen, desto höher ist in der Regel die Beweiskraft. Da z.B. in-vivo- und in-vitro-Daten bei der Entscheidung ein höheres

361 EuG, Urteil vom 11.07.2007, Rs.T-229/04, Schweden/Kommission (Paraquat), ECLI:EU:T:2007:217, Rn. 161. S.a. BVerwG, NVwZ-RR 1990, 134 (136), zur Zulassung paraquathaltiger Pflanzenschutzmittel: „Das VG hat auch die Vorschrift des $\int 8$ Abs. 1 Nr. 3 PflSchG 1968 unrichtig ausgelegt. Es hat auch in diesem Zusammenhang verkannt, daß eine Zulassung nicht erst dann zu versagen war, wenn für unvertretbare schädliche Auswirkungen eine hinreichende Wabrscheinlichkeit bestand, sondern die Zulassung umgekehrt voraussetzte, daß unvertretbare schädliche Auswirkungen mit an Sicherbeit grenzender Wabrscheinlichkeit ausgeschlossen waren".

362 S.o. A II.2., C I.4.

363 S. z.B. ECHA (RAC), CLH Report, (Fn. 184), S. 98, betreffend die Bewertung der Kanzerogenität von Glyphosat. 
Gewicht als QSAR ${ }^{364}$ - oder andere Berechnungsmethoden haben, ${ }^{365}$ gelten die Ergebnisse aus direkter Untersuchung als höherwertig im Vergleich zu Rückschlüssen aus Statistiken und Rechenoperationen.

All dies ändert allerdings nichts daran, dass hier nicht lediglich hypothetische Annahmen, sondern eine Reihe von gegensätzlichen Positionen existierten, die vernünftige Zweifel an der Sicherheit von Glyphosat begründen können. Das deutsche BfR räumte selbst im finalen Renewal Assessment Report ein, dass es Studien gäbe, die einen Zusammenhang zwischen der Glyphosat-Exposition und dem Auftreten des Non-Hodgkin-Lymphom aufzeigten. Es erklärte diesen Zusammenhang aber für „in den meisten Fällen“ (!) nicht signifikant und berief sich darauf, dass nicht ausgeschlossen werde könne, dass Beistoffe die Korrelation bewirkt hätten. ${ }^{366}$ Genaueres weiß man aber nicht. Epidemiologischen Studien, die den Zusammenhang ebenfalls bestätigten, hatte das BfR im Renewal Assessment Report (RAR) wegen fehlender Zusatzinformationen betreffend Rauchverhalten, Exposition und Vorerkrankungen der untersuchten Personen als „non reliable“ qualifiziert, obwohl diese Zusatzinformationen tatsächlich erhoben worden waren. Dies räumte das BfR zwar später ein, beharrte jedoch darauf, dass darüber jedenfalls „,in den Publikationen nicht ausreichend berichtet" worden sei. ${ }^{367}$ Auch das Joint FAO/WHO-Gremium JMPR räumte ein, dass es einen „gewissen Nachweis“ für den Zusammenhang gäbe, der aber nur durch statistische Meta-Analysen erbracht sei, während eine große Kohortenstudie keinen Zusammenhang ergeben habe. ${ }^{368}$ Die Studie wird zum Teil wegen ihrer zu kurzen Laufzeit kritisiert.

Die Auffassung der befassten Behörden, wonach Glyphosat gesundheitlich unbedenklich bzw. „wahrscheinlich nicht kanzerogen“ sei, weil die Studien, auf die sie ihre Position stützten, den Studien der Gegenseite qualitativ überlegen seien, beruht auf einer grundlegenden Verkennung des Vorsorgeprinzips. Selbst wenn nämlich Kohortenstudien ein grundsätzlich höheres Gewicht als andere Studien haben, ergibt sich daraus nicht die Irrelevanz von statistischen Analysen, die zu gegenteiligen Ergebnissen gelangen, soweit für die Abweichung kein plausibler Grund gefunden werden kann. Wie das oben genannte Beispiel aus dem Bereich des RAR zeigt, ist der Standpunkt von BfR und EFSA, man habe sich für die Methode des "weight of evidence approach" entschieden, nicht einmal überzeugend angewandt worden, denn es werden nicht ins gewünschte Bild passende Studien mit der Behauptung ausgeschlossen, sie seien nicht verlässlich, da bestimmte Angaben nicht hinreichend deutlich würden, ohne dass interessiert, ob es sich dabei um Darstellungs- oder um Sachmängel handelt. Da maßgebliche Studien bis zuletzt in Teilen geheim gehalten wurden, ${ }^{369}$ bleibt un-

364 Bewertungsmodelle des Typs "Quantitative structure-activity relationship" (Quantitative Struktur-Wirkungsbeziehung) berechnen Risikopotentiale anhand bestimmter prädikativer Variablen.

$365 E C H A$, Beweiskraft der Daten, abrufbar unter: https://echa.europa.eu/de/support/regist ration/how-to-avoid-unnecessary-testing-on-animals/weight-of-evidence (15.06.2019).

366 S.o. C I.3.a).

367 BT-Drs. 18/8408, (Fn. 37), S. 10.

368 S.o. C I.3.a).

369 S.o. C I.2.c). 
überprüfbar, ob nicht auch Studien, die kein Risiko erkannten, in irgendwelchen Punkten als „non reliable“ hätten eingestuft werden müssen. Hinzu kommt, dass die Bewertung durch BfR und EFSA mit Blick auf die Monsanto Papers, festgestellte Plagiate aus Unternehmensdokumenten und dergleichen nicht mehr als objektiv und unabhängig angesehen werden kann. ${ }^{370} \mathrm{Nach}$ hier vertretener Auffassung verlangt das Vorsorgeprinzip es aber auch, dass eine nicht unabhängig und objektiv durchgefübrte Bewertung in angemessener Form wiederholt wird.

Unter den hier gegebenen Umständen - schnelles Voranschreiten der wissenschaftlichen Entwicklung speziell bei Glyphosat (Erwägungsgrund $19 \mathrm{f}$. DurchführungsVO zur Wiedergenehmigung), Existenz einer ernstzunehmenden Mindermeinung, Annahme eines Risikos durch die maßgebliche WHO-Institution, statistische Belege für ein Risiko, in ihrer Relevanz bestrittene Kohortenstudien, die ein Risiko nahelegen, Ergebnisse gerichtlicher Beweisaufnahmen, fehlende Unabhängigkeit der Bewertung bei gleichzeitiger Geheimhaltung maßgeblicher Studien, die ein Risiko als „unwahrscheinlich" einstufen - ist die Möglichkeit eines Gesundheitsrisikos alles Andere als auszuschließen. Infolgedessen hätte das Vorsorgeprinzip mindestens verlangt, effektiv risikomindernde Vorgaben zum Gesundheitsschutz in die DurchführungsVO zur Wiedergenehmigung von Glyphosat aufzunehmen. Stattdessen enthält die DurchführungsVO aber nur allgemein gehaltene Aufforderungen an die Mitgliedstaaten, bei der Zulassung der Anwendungsmittel auf bestimmte Belange besonders zu achten. ${ }^{371}$

Soweit es mögliche Umweltrisiken betrifft, lässt die Bewertung selbst Problembereiche erkennen. Es fehlt aber an einer nachvollziehbaren Behandlung der Frage, ob die festgestellten schädlichen Auswirkungen insbesondere auf die Biodiversität „unannehmbar" im Sinne des europäischen Rechts sind. Es fehlt insoweit bereits an Kriterien. ${ }^{372}$ Auch hier gelangt die DurchführungsVO nicht über vage Aufforderungen an die Mitgliedstaaten hinaus, sich dem jeweiligen Problempunkt im Verfahren der Wiederzulassung der Pflanzenschutzmittel zu widmen. Dazu ist es dann aber, wie das Beispiel Deutschland zeigt, selbst eineinhalb Jahre nach der Wirkstoff-Neugenehmigung nicht gekommen.

Insgesamt hat die Europäische Kommission ihre Aufgabe der Risikominderung nahezu vollständig an die Mitgliedstaaten delegiert, d.h. sie in ein Folgeverfahren (Pflanzenschutzmittel-Zulassung) verschoben, dass trotz gewisser Überschneidungen mit dem Genehmigungsverfahren anderen Zwecken als das Verfahren der Wirkstoffgenehmigung dient und nicht zur Neubewertung der Genehmigungsentscheidung führen kann. Damit wurde zugleich die Gefahr begründet, dass sich die Mitgliedstaaten bei der Pflanzenschutzmittel-Zulassung wiederum umgekehrt darauf berufen, dass der Wirkstoff genehmigt sei. Selbst wenn die Zusammenarbeit mit den Mitgliedstaaten im Komitologieverfahren schwierig war, ein Teil des Problems also nicht im Verhalten der Kommission liegen sollte, ändert dies nichts daran, dass die Kommission

370 S.o. C I.4.

371 S.o. C I.3.b).

372 S. auch die Kritik des Europäischen Parlaments in seiner Resolution vom 16.01.2019, (Fn. 95), sub BM. 
ihrer originären Verantwortung für die Sicherheit der Wirkstoffgenehmigung nicht gerecht geworden ist und das Vorsorgeprinzip verletzt hat.

\section{Individualrechte}

\section{Umweltinformationsrechte}

Grundsätzlich gewährt das Unionsrecht Individuen und Verbänden ein Recht auf Zugang zu Umweltinformationen. Die impulsgebenden Maßstäbe setzt dabei ein völkerrechtlicher Vertrag, die Århus-Konvention, die in das EU-Umweltinformationsrecht, d.h. die Århus-Richtlinie 2003/4373 und die Århus-Verordnung 1367/2006,374 übersetzt wurde. Daraus ergeben sich die folgenden grundlegenden Regeln: Es besteht ein Recht auf Zugang zu Umweltinformationen, das aber unter Umständen hinter andere Interessen wie insbesondere den Schutz der Geschäfts- und Unternehmensgeheimnisse zurücktreten muss. Dies ist im Einzelfall durch Abwägung zu klären. Jedoch wird nach Art. 6 Abs. 1 Satz 1 Århus-Verordnung und Art. 4 Abs. 2 ÅrhusRichtlinie bei „Informationen über Emissionen in die Umwelt“ unwiderleglich vermutet, dass das öffentliche Interesse an der Veröffentlichung überwiegt. Die Ratio dieser Regelung wird darin gesehen, dass jemand, der andere dazu zwingt, von ibm freigesetzte Stoffe in seinen Körper aufzunebmen, sich nicht auf Gebeimbaltung berufen können soll. ${ }^{375}$ Eine Verstärkung erfahren die Umweltinformationsrechte durch Art. 8 EMRK (i.V.m. Art. 6 Abs. 3 EUV), der ein primärrechtliches Individualrecht auf Information über gesundheitsrelevante Umstände vermittelt. ${ }^{376}$

Schon im Zusammenhang mit der Erstgenehmigung des Wirkstoffs Glyphosat war Streit über den Zugang zu umweltrelevanten Informationen entstanden. Im Fall Stichting Greenpeace Nederland und PAN Europe ${ }^{377}$ hatten die Kommission und das BfR 2011 einen Antrag auf Zugang zu Dokumenten teilweise unter Hinweis auf Rechte der Unternehmen an geistigem Eigentum abgelehnt. Es ging um die Zusammensetzung der zur Genehmigung eingereichten Wirkstoffkombinationen nebst Verunreinigungen. Die Behörden stellten sich auf den Standpunkt, dass es sich hierbei nicht um „Informationen über Emissionen in die Umwelt“ handele. Dagegen klagten die beiden Verbände unter Berufung auf Art. 6 Abs. 1 Satz 1 Århus-Verordnung. So-

373 Richtlinie 2003/4/EG des Europäischen Parlaments und des Rates vom 28. Januar2003 über den Zugang der Öffentlichkeit zu Umweltinformationen und zur Aufhebung der Richtlinie 90/313/EWG des Rates, ABl. (EU) L 41 v. 14.02.2003, S. 26.

374 Verordnung (EG) Nr.1367/2006 Des Europäischen Parlaments und des Rates vom 6. September 2006 über die Anwendung der Bestimmungen des Übereinkommens von Århus über den Zugang zu Informationen, die Öffentlichkeitsbeteiligung an Entscheidungsverfahren und den Zugang zu Gerichten in Umweltangelegenheiten auf Organe und Einrichtungen der Gemeinschaft, ABl. (EU) L 264 v. 25.09.2006, S. 13.

375 Wegener, ZUR 2017, S. 146.

376 Marauhn/Thorn, in: Dörr/Grote/Marauhn (Hrsg.), Kap. 16, Rn. 31 f.; Richter, in: Dieselben, Kap. 9, Rn. 35.

377 EuGH, Rs. C-673/13 P, Kommission/Stichting Greenpeace Nederland und PAN Europe, ECLI:EU:C:2016:889. Die Sache wurde an das EuG zurückverwiesen. 
wohl das EuG als auch der EuGH gaben ihnen im Wesentlichen Recht. Ähnlich endeten die Verfahren auf der Basis von Art. 4 Abs. 2 Århus-Richtlinie in der Sache Bijenstichting vor dem EuGH (2016) ${ }^{378}$ und in den Rechtssachen Tweedale sowie Hautala vor dem EuG (beide 2019). ${ }^{379}$ Nach Auffassung beider Gerichte kommt es weder auf die Art noch die Quelle der Emission an. Insbesondere dürfe der Emissionsbegriff der Århus-Verordnung nicht auf denjenigen von Art. 3 Nr. 4 Industrieemissionen-Richtlinie 2010/75/EU verengt werden. Deshalb falle auch das Freisetzen von Produkten oder Stoffen wie Pflanzenschutzmitteln oder Biozid-Produkten und in diesen Produkten enthaltenen Stoffen in die Umwelt unter den Begriff „Emissionen in die Umwelt“. Art. 6 Abs. 1 Århus-Verordnung (entsprechend Art. 4 Abs. 2 ÅrhusRichtlinie) erfasse sowohl „tatsächliche“ als auch „vorhersehbare“, nicht aber nur hypothetische Emissionen. Nicht hypothetisch seien insbesondere Emissionen, die beantragte Verwendungen erwarten lassen. Dass ein Stoff hergestellt oder allgemein in Verkehr gebracht wird, reiche aber nicht. Ein bloßer Bezug der gewünschten Informationen zu Emissionen genüge ebenfalls nicht, weil nur „Informationen über Emissionen" offenzulegen seien. Zu diesen gehörten Angaben über die Art, Zusammensetzung, Menge, Zeitpunkt und Ort der Emissionen eines Stoffes oder Anwendungsprodukts, aber auch Daten über die Auswirkungen dieser Emissionen auf die Umwelt sowie Informationen über die Rückstände in der Umwelt nach der Anwendung des fraglichen Produkts und Studien zur Messung der Stoffdrift bei dieser Anwendung. Soweit es den Zugang zu Studien im Speziellen betrifft, erkannte der EuGH

- „Studien, mit denen die Toxizität, die Auswirkungen und andere Gesichtspunkte eines Produkts oder Stoffes unter den ungünstigsten realistischen Bedingungen, die vernünftigerweise erwartet werden können, ermittelt werden sollen, und

- Studien, die unter Bedingungen durchgeführt werden, die der normalen landwirtschaftlichen Praxis so nahe wie möglich kommen und in dem Gebiet vorherrschen, in dem dieses Produkt oder dieser Stoff angewandt wird,“

ausdrücklich als „Informationen über Emissionen“ an. ${ }^{380}$ Testfrage für den EuGH ist, ob die Öffentlichkeit den Zugang zu den Informationen benötigt, um nachzuprüfen, ob die Emissionen zutreffend bewertet wurden, und um damit „wirksam am Entscheidungsprozess im Umweltbereich teilnehmen zu können“". ${ }^{381}$ Grundrechtliche Positionen der Unternehmen oder das TRIPS-Abkommen stünden dieser Interpretation des Emissionsbegriffs der Århus-Regelungen nicht entgegen. Der EuGH hob hervor, dass Art. 63 Abs. 3 VO 1107/2009 die Vertraulichkeit von Informationen nur

378 EuGH, Rs. C-442/14, Bayer CropScience SA-NV, Stichting De Bijenstichting gegen College voor de toelating van gewasbeschermingsmiddelen en biociden, ECLI:EU:C:2016:890.

379 EuG, Rs. T-716/14 und 329/17, Antony C. Tweedale gegen EFSA, ECLI:EU:T:2019:141; Rs. 329/17, Heidi Hautala u.a., ECLI:EU:T:2019:142.

380 EuGH, Bayer, (Fn. 378), Rn. 91.

381 EuGH, Kommission/Stichting Greenpeace Nederland und PAN Europe, (Fn. 377), Rn. 80. 
„unbeschadet der Richtlinie 2003/4/EG ... über den Zugang der Öffentlichkeit zu Umweltinformationen" gewährt.

Legt man die Maßstäbe dieser Rechtsprechung an die zurückgehaltenen Informationen im Glyphosat-Wiedergenehmigungsverfahren an, erweist sich die Argumentation der europäischen Behörden als unhaltbar. Sie erklären zwar ihre Bewertung hinsichtlich der möglichen Kanzerogenität von Glyphosat als überlegen, weil sie über bessere Studien (insbesondere Unternehmensstudien) verfügten, - die müssten allerdings gemäß Art. 63 VO 1107/2009 vertraulich bleiben. ${ }^{382}$ Damit werden gerade die relevanten Studien zurückgehalten, um Betriebsgeheimnisse der Hersteller zu schützen, und es wird unmöglich zu überprüfen, ob Studien, die Risiken verneinen, tatsächlich hochwertig und valide, Studien, die Risiken bejahen, jedoch fehlerhaft und nicht-repräsentativ sind. Dass diese zentralen Eckpunkte der Århus-Urteile zur Offenlegung von Studien nicht in der Realität des Genehmigungsverfahrens angekommen sind, hat die „Portier-Kontroverse“ ${ }^{383}$ anschaulich gezeigt. Denn bis zum Schluss wurde nur Zugang zu den Rohdaten gewährt. Das kann - vorausgesetzt, es werden Anträge auf Zugang zu vertraulichen Studien gestellt - keinen Bestand vor Art. 6 Abs. 1 Århus-Verordnung bzw. Art. 4 Abs. 2 Århus-Richtlinie haben.

Die Prognose, dass die Unternehmen weiter für die Begrenzung des Zugangs „kämpfen“ würden, ${ }^{384}$ trifft sicherlich zu. Durch seine Urteile von 2016 stellt der EuGH aber jenes „bilaterale Verhältnis“385 infrage, das bislang das Genehmigungsverfahren kennzeichnete: Die Hersteller testen und bewerten die von ihnen entwickelten Produkte selbst und legen die Ergebnisse den zuständigen Behörden vor. Bleiben die für eine Bewertung der Tests und der Validität ihrer Ergebnisse nötigen Detailinformationen vertraulich, wird die öffentliche Debatte über die Risiken der zu genehmigenden Produkte „wesentlich behindert“ und kommt es nur noch auf die Kontrollkompetenz und den Kontrollwillen der Behörden an. ${ }^{386}$ Dass darauf nicht blind vertraut werden kann, haben die Monsanto Papers offenbart.

Die Reformvorschläge der Kommission zur Steigerung der Transparenz ${ }^{387}$ dürften die Lage nicht grundlegend verbessern. Denn auch sie folgen nicht dem Ansatz des EuGH, alle bewertungsrelevanten Informationen, die Emissionen in die Umwelt betreffen, auf Antrag zugänglich zu machen, sondern bleiben - mit Verbesserungen im Detail - dem herkömmlichen Abwägungsansatz „Transparenz versus Betriebsgeheimnisse“ verhaftet.

\section{Grund- und Menschenrechte}

Im Folgenden geht es um die Frage, ob die Wiedergenehmigung von Glyphosat aufgrund Durchführungsverordnung (EU) 2017/2324 der Kommission und die Folge-

382 S.o. zur entsprechenden Stellungnahmen der Kommission C I.3.a).

383 S.o. C I.4.a).

384 Wagner, EuZW 2017, S. 98.

385 Wegener, ZUR 2017, S. 151; zuvor bereits ders., ZUR 2014, S. 35.

386 Ibid.

387 S.o. C I.2.c). 
maßnahmen der Mitgliedstaaten, insbesondere Deutschlands, Grund- und Menschenrechte verletzen könnten. Sie kann hier nicht abschließend geklärt werden, sondern nur in ihren wesentlichen Grundrissen angedacht werden. Die Ausführungen konzentrieren sich auf zentrale Aspekte, insbesondere die Relevanz und Reichweite der in Betracht kommenden europäischen Prinzipien und Grundrechte.

\section{a) Vorsorgeprinzip und Pflicht zur Sicherstellung eines "hohen Gesundheitsniveaus" (Art. 35 Satz 2 GrCh) als Leitprinzipien der Grundrechtsinterpretation}

Wie gezeigt wurde, bestehen gewichtige Gründe für die Annahme, dass das Vorsorgeprinzip bei der Wiedergenehmigung von Glyphosat missachtet wurde. ${ }^{388}$ Die insoweit relevanten Bestimmungen vermitteln zwar keine subjektiven Rechte, definieren aber Inhalt und Ausmaß der von den zuständigen Institutionen zu beachtenden Sorgfalt. Grundrechte, die auf den individuellen Gesundheitsschutz zielen, sind in jedem Falle so auszulegen, dass auch dem Vorsorgeprinzip Rechnung getragen wird.

Art. 35 Satz 2 der Europäischen Grundrechtecharta (GrCh) verpflichtet die Institutionen, bei der Festlegung und Durchführung der Politik und Maßnahmen der Union ein „hohes Gesundheitsniveau“ sicherzustellen. Diese Verpflichtung bindet alle Einrichtungen der Union und ebenso die Mitgliedstaaten, soweit diese Unionsrecht durchführen (Art. $51 \mathrm{GrCh}$ ). Sie greift die Querschnittsverpflichtung aus Art.9 AEUV zum Gesundheitsschutz wie auch die gleichlautende Verpflichtung aus Art. 168 Abs. 1 UAbs. 1 AEUV auf und schlägt sich nicht zuletzt in der Zielsetzung der europäischen Pflanzenschutzverordnung nieder, ein „hohes Schutzniveau für die Gesundheit von Mensch und Tier und für die Umwelt" neben dem besseren Funktionieren des Binnenmarktes sicherzustellen (Art. 1 Abs. 3 VO 1107/2009). Inhaltlich stützt sich Art. 35 GrCh nicht nur auf Art. 168 Abs. 1 UAbs. 1 AEUV, sondern auch auf Art. 11 und 13 der Europäischen Sozialcharta (EuSozialCh). ${ }^{389}$ Während allerdings Art. 35 Satz 1 GrCh (Zugang zur Gesundheitsvorsorge und ärztlichen Versorgung) als „echtes Grundrecht" betrachtet wird, soll es sich bei Satz 2 (Sicherstellung eines hohen Gesundheitsschutzniveaus) nur um eine Zielbestimmung i.S. eines Optimierungsgebots handeln. ${ }^{390}$ Das ändert allerdings nichts daran, dass Art. 35 Satz 2 GrCh den EU-Institutionen und den Mitgliedstaaten bei der Durchführung des EURechts eine rechtlich bindende Verpflichtung auferlegt. Deshalb kann und muss er zur Auslegung und Inhaltsbestimmung von Schutzverpflichtungen herangezogen werden, die sich aus Art. 35 Satz $1 \mathrm{GrCh}$, aber auch aus anderen subjektiven Rechten im Zusammenhang mit der Gesundheit ergeben. Dabei können der Zielvorgabe, ein „hohes Gesundheitsniveau“ sicherzustellen, zwar keine konkreten Verpflichtungen zum Ergreifen bestimmter Maßnahmen entnommen werden. Art. 35 Satz 2 GrCh wird aber verletzt, wenn durch Tun oder Untätigkeit dem Ziel entgegengehandelt oder

388 S.o. E I.

389 Siehe die Erläuterungen zur Charta der Grundrechte, ABl. (EU) C 303 v. 14.12.2007, S. 17, 27.

390 Krajerwski, in: Pechstein/Nowak/Häde, Art. 35 GrCh, Rn. 5, 17 (m.w.N.). 
es ignoriert wird. Zu beachten ist auch, dass das hohe Gesundheitsniveau „sichergestellt" und nicht lediglich angestrebt werden muss.

\section{b) Recht auf Gesundheitsvorsorge (Art. 35 Satz 1 GrCh)}

Art. 35 Satz 1 GrCh vermittelt Individuen im Anwendungsbereich der Grundrechtecharta (Art. $51 \mathrm{GrCh}$ ) ein europäisches Grundrecht auf Zugang zur Gesundheitsvorsorge und ärztlichen Versorgung. Dabei wird der Begriff der „Gesundheitsvorsorge“ in Anlehnung an Art. 11 Abs. 3 EuSozialCh so verstanden, dass er präventive Maßnahmen erfordert, die Krankheiten verhindern oder (schon eingetretene) begrenzen. Darunter fallen nicht nur Maßnahmen wie Impfprogramme, sondern auch Maßnahmen zum Schutz der Umwelt, wenn Umweltverschmutzungen zu Krankheiten führen können. ${ }^{391}$ Zwar verpflichtet dies die Institutionen in der Regel nicht zu bestimmten Maßnahmen, sondern lässt ihnen einen weiten Gestaltungsspielraum. Selbst bei komplexen Umweltproblemen, die nicht sogleich beseitigt werden können, stehen die Staaten aber in der Pflicht, alle Anstrengungen zu unternehmen, um das Ziel innerhalb vernünftiger Zeit zu erreichen. Dazu gehören auch ein angemessener Kontrollmechanismus und nötigenfalls vorsorgliche Maßnahmen. ${ }^{392}$ Der Ausschuss zur Sozialcharta geht davon aus, dass Art. 11 EuSozialCh die gesundheitsbezogenen Aussagen des Art. 8 EMRK ergänzt. ${ }^{393}$ Entsprechendes gilt dann auch für Art. 35 Satz 1 GrCh, der in Anlehnung an Art. 11 EuSozialCh konzipiert wurde.

Ohne dass die Frage hier bis in die Details geklärt werden kann, haben Individuen, die mit Glyphosat oder glyphosathaltigen Mitteln in Berührung kommen können, ein Recht darauf, dass die Union und ihre Mitgliedstaaten beim Handeln aufgrund der europäischen Pflanzenschutzverordnung 1107/2209 diejenigen Vorsorgemaßnahmen treffen, die nötig sind, um Krankheiten (wie z.B. Erkrankungen am Non-HodgkinSyndrom) zu verhindern, d.h. das Vorsorgeprinzip zu beachten. Betroffen sein könnten nicht nur Personen, die beruflich mit einem Pestizid in Berührung kommen oder in der Nähe behandelter Flächen leben, sondern auch jene, die glyphosatbelastete Nahrungsmittel zu sich nehmen. Insoweit kommt es darauf an, dass die festgesetzten Höchstmengen für Rückstände nicht nur dem Vorsorgeprinzip entsprechen, sondern auch dem Ziel, ein „hohes Gesundheitsniveau“ sicherzustellen. Das setzt weitaus mehr als das Unterlassen schädigender Eingriffe voraus und dürfte insbesondere auch die Sicherstellung gesunder Lebensmittel als Basis für ein hohes Gesundheitsniveau umfassen. Gesund sind Lebensmittel aber nicht schon dann, wenn sie festgelegte Höchstwerte an Pestizid-Rückständen gerade noch einhalten, sondern wenn alles getan wird, um deren Anreicherung in Lebensmitteln auszuschließen. Letztlich wird zwischen dem Recht auf Gesundheitsvorsorge (Art. 35 Satz $1 \mathrm{GrCh}$ ) und anderen öffentlichen und privaten Interessen an der Nutzung von Pestiziden abzuwägen sein. Ein „hohes

391 European Social Rights Committee, Entscheidung vom 11.09.2012 (Merits), Beschwerdenr. 72/2011, International Federation of Human Rights Leagues (FIDH) v. Greece, Rn. 51.

392 Ibid., Rn. 129, 133, 150.

393 Ibid., Rn. 51. 
Gesundheitsniveau“ ist aber unvereinbar mit der Aussage, dass „ein wenig Glyphosat im Körper" in einer hochtechnisierten Gesellschaft unvermeidlich, sozialadäquat und jedenfalls aus übergeordneten Gründen hinzunehmen sei. Das gilt jedenfalls dann, wenn wie im Falle von Glyphosat oder glyphosathaltiger Mittel die Exposition nur „wahrscheinlich nicht kanzerogen“ ist, die Rückstandshöchstmengen in höchstem Maße umstritten oder unsicher sind und auch die Unabhängigkeit der Bewertung erheblichen Zweifeln unterliegt. ${ }^{394}$

c) Recht auf Schutz vor schwerwiegenden Umweltbeeinträchtigungen (Art. 7 GrCh, Art. 8 EMRK)

Gemäß Art. 7 GrCh, der im Zweifel wie Art. 8 EMRK zu interpretieren ist (Art. 52 Abs. 3 GrCh), können schwerwiegende Umweltbelastungen oder das Verschweigen relevanter Umweltinformationen das Recht auf den ungestörten Genuss der Privatund Wohnsphäre verletzen. Bedeutsam ist insoweit die Rechtsprechung des EGMR, wonach schwerwiegende Umweltbelastungen das Wohlbefinden Einzelner beeinträchtigen, so dass Fehlverhalten der Behörden beim Umgang mit diesen Belastungen zur Verletzung von Art. 8 EMRK (Recht auf den ungestörten Genuss der Privat- und Wohnsphäre) führen kann, ${ }^{395}$ und zwar selbst dann, wenn keine schwerwiegende Beeinträchtigung der Gesundheit drobt bzw. dies umstritten ist. ${ }^{396}$ Weil es im Kern um Lebensqualität geht - der EGMR spricht von einem „Recht auf Schutz der Wohnund Privatsphäre gegen schwerwiegende Umweltmissstände“, 397 kommt es nicht darauf an, dass ein Zusammenhang zwischen bestimmten Gesundheitsbeeinträchtigungen und der Umweltverschmutzung nachgewiesen werden kann. ${ }^{398}$ Vielmehr verletzt der Staat seine Schutzpflicht aus Art. 8 EMRK schon dann, wenn er keine faire Abwägung der widerstreitenden Interessen (Art. 8 Abs. 2 EMRK) vornimmt und passiv bleibt, wenn schwerwiegende Umweltverschmutzung den Menschen das Leben im privaten Wohnumfeld verleidet - und damit auch in aller Regel die Gesundheitsrisiken für sie erhöht. Grundsätzlich besitzt der Staat einen weiten Gestaltungsspielraum bei der Abwägung zwischen Umwelt- und widerstreitenden Belangen; dieser verengt sich jedoch stark, wenn es um „intime Aspekte“ des Privatlebens geht. Das erfasst z.B. Situationen, in denen eine Person wegen Fluglärms nicht schlafen kann, ${ }^{399}$ erst recht dann aber auch den Fall, dass sie gezwungen wird, Pestizide in den eigenen Körper

394 S.o. C I.4.

395 EGMR, Std. Rspr. S. etwa No. 16798/90, López Ostra, insb. Rn. 51 ff.; No. 14967/89, Guerra and Others, Rn. 56 ff.; No. 55723/00, Fadeyeva, Rn. 79 ff.; No. 36022/97, Hatton, Rn. 96; Nos. 53157/99 u.a., Ledyayeva, Dobrokhotova, Zolotareva and Romashina, Rn. $89 \mathrm{ff}$.

396 EGMR, No. 16798/90, López Ostra, Rn. 51: "Naturally, severe environmental pollution may affect individuals' well-being and prevent them from enjoying their homes in such a way as to affect their private and family life adversely, without, however, seriously endangering their health." Ebenso EGMR, No. 14967/89, Guerra and Others, Rn. 60.

397 EGMR, Ledyayeva, Dobrokhotova, Zolotareva and Romashina, (Fn. 395), Rn. 110.

398 Ibid., Rn. 95.

399 EGMR, Hatton, (Fn. 395), Rn. 102 f. 
aufzunehmen. Um seiner Schutzpflicht zu genügen, muss der Staat als Resultat der Güterabwägung angemessene Regelungen in Kraft setzen und für die Überwachung der Rechtsbefolgung sorgen. Missachtet er seine eigenen Standards, ist dies ein wichtiges, aber nicht notwendiges Indiz für die Verletzung von Art. 8 EMRK. Die betroffenen Individuen ihrerseits haben ein Recht darauf, über bestehende Risiken informiert zu werden. ${ }^{400} \mathrm{Um}$ verletzt zu sein, müssen sie allerdings darlegen können, dass sie „direkt und schwerwiegend“ betroffen sind. ${ }^{401}$

Entscheidend ist also die Frage, ob Personen, die glyphosathaltigen Pestiziden ausgesetzt sind, weil sie beruflich damit umgehen, in der Nähe behandelter Flächen leben oder Lebensmitteln bzw. Trinkwasser mit Glyphosatrückständen ausgesetzt sind, „direkt und schwerwiegend“ betroffen sind. An der Direktheit der Einwirkung kann kein Zweifel bestehen, soweit Glyphosat/AMPA in den menschlichen Körper gelangt. Unter welchen Umständen liegt aber auch eine hinreichend schwerwiegende Belastung vor? Argumentiert werden könnte, dass die Einbringung von Pestiziden in den menschlichen Körpers eine besonders intrusive Art der Inanspruchnahme ist, die generell als schwerwiegend gelten muss. Dagegen könnte eingewandt werden, dass die Einhaltung von regulär festgelegten Grenzwerten und Höchstmengen die maßgebliche Trennlinie zwischen schwerwiegenden und nicht schwerwiegenden Beeinträchtigungen durch Pestizideinwirkung markiert. Dieser Einwand kann aber dann nicht durchgreifen, wenn, wie hier, die Grenzwerte fragwürdig zustande gekommen sind, sie insbesondere ständig schwanken, nicht alle Risiken abgeklärt sind ${ }^{402}$ oder das Vorsorgeprinzip verletzt wurde. Unter solchen Umständen schließt die Einhaltung von Grenzwerten eine schwerwiegende Umweltbeeinträchtigung, wie sie beim Eintrag von Pestiziden in den menschlichen Körper grundsätzlich anzunehmen ist, nicht aus.

\section{d) Recht auf einen pestizidfreien Körper und Schutz vor Kontamination (Art. 3, 7 GrCh, Art. 8 EMRK)}

Von Bedeutung ist die „exzessive Intimität“ der Einwirkung und ihre Unausweichlichkeit aber auch für sich genommen, d.h. ohne dass der „Umweg“ über eine schwerwiegende Umweltbeeinträchtigung genommen werden müsste. Gelangen nämlich Pestizide in Nahrung und Trinkwasser, Boden und Luft, wird wie z.B. im Falle glyphosathaltiger Pestizide der Körper der Person und damit das „Intimste“, was sie hat, im Interesse Dritter in Anspruch genommen. Der menschliche Körper nimmt infolge der Kontamination von Lebensmitteln, Wasser, des Bodens oder der Luft Pflanzenschutzmittel auf, die dort nicht hingehören. Selbst wenn die Dosis minimal wäre, Glyphosat zu 100\% wieder aus dem menschlichen Körper ausgeschieden würde und bis dahin auch keine Gesundheitsschädigungen ausgelöst würden, ${ }^{403}$ also keine

400 EGMR, Guerra, (Fn. 396), Rn. 60.

401 EGMR, Hatton, (Fn. 395), Rn. 95: "There is no explicit right in the Convention to a clean and quiet environment, but where an individual is directly and seriously affected by noise or other pollution, an issue may arise under Article 8".

402 S.o. E II.2.b).

403 S.o. A II.2. 
Beeinträchtigung der körperlichen Unversehrtheit (Art. $3 \mathrm{GrCh}$ ) vorliegt, stellt sich die grundsätzliche Frage, ob diese intrusive Form der Inanspruchnahme nicht die Selbstbestimmung über den eigenen Körper und damit das Recht auf Schutz der Privatsphäre (Art. 7 GrCh, Art. 8 EMRK) beeinträchtigt.

Im Grunde ähnelt die hier gegebene Situation der Aufbringung von Keimen auf Lebensmittel, wie sie z.B. entsteht, wenn Verkaufspersonal Geld von der einen Kundin kassiert, um im nächsten Moment Lebensmittel mit bloßen Händen für den nächsten Kunden zu ergreifen. Die Aufbringung von Keimen, gefährlich oder nicht, gilt z.B. im deutschen Lebensmittelrecht als „nachteilige Beeinflussung“. Eine solche Beeinflussung liegt nach der deutschen Lebensmittelhygieneverordnung ausdrücklich auch dann vor, wenn die einwandfreie hygienische Beschaffenheit von Lebensmitteln durch Pflanzenschutzmittel durch den bloßen Auftrag beeinträchtigt wird. ${ }^{404}$ In beiden Fällen (Glyphosat in Backwaren, Keime auf Backwaren) mag im Einzelfall strittig sein, ob die Kontamination sich konkret schädlich auf die Gesundheit auswirkt, eine nachteilige Beeinflussung liegt aber in beiden Fällen vor.

Dass das Recht auf den Schutz der Privatsphäre aus Art. 8 EMRK auch das Selbstbestimmungsrecht des Einzelnen über seinen Körper als einen notwendigen Bestandteil des Privatlebens umfasst, ist heute in der Lehre und auch in der Rechtsprechung des EGMR in Bezug auf einzelne Fallgruppen anerkannt. ${ }^{405}$ Es geht dabei weder um die Beeinträchtigung der körperlichen Unversehrtheit, die die EMRK außerhalb von Art. 3 und im Unterschied zu Art. 3 GrCh nicht unmittelbar schützt, noch um ein Recht auf eine gesunde Umwelt, das nur bei schwerwiegenden Beeinträchtigungen Art. 8 EMRK, Art. 7 GrCh unterfällt. Anknüpfungspunkt ist vielmehr, dass das Individuum Stoffe in seinen Körper hineinlassen muss, die dort nichts zu suchen haben, d.h. um Integritätsschutz vor auch nur möglicherweise riskanten Stoffen im Sinne eines Selbstbestimmungsrechts über den eigenen Körper - „habeas corpus“ in seiner eigentlichsten und innerlichsten physischen Form.

Man könnte zwar einwenden, es sei in der heutigen Zeit unmöglich, stoffliche Einwirkungen auf Menschen aus Industrie, Verkehr oder Landwirtschaft auszuschließen. Das ist aber allenfalls eine Frage der Güterabwägung zwischen den hier kollidierenden Rechtsgütern. Wenn der Staat Zulassungen über das Inverkehrbringen und die Anwendung von Pestiziden erteilt, die sich im Körper unbeteiligter Dritter gegen deren Willen mindestens vorübergehend messbar niederschlagen, ist der Schutzbereich des Art. 8 EMRK bzw. Art. $7 \mathrm{GrCh}$ nach hier vertretener Auffassung berührt. Die Schutzverpflichtung entspricht dabei nach Art und Ausmaß dem Selbstbestimmungsrecht des betroffenen Individuums am eigenen Körper bzw. dem „Recht auf einen pestizidfreien Körper". Deshalb darf der Staat den Individualschutz nicht von vornherein auf Fälle der maximal zulässigen täglichen Höchstmenge eines bestimmten Stoffes beschränken. Ein Eingriff liegt in der messbaren unfreiwilligen Kontamination.

$404 \$ 2$ Ziffer 1 Lebensmittelhygieneverordnung vom 08.08.2007 in der Fassung vom 21.06.2016.

405 S. Marauhn/Thorn, in: Dörr/Grote/Marauhn, Kap. 16, Rn. 17, 33, 73 f. 


\section{Prozessuale Durchsetzung und laufende Verfahren}

Im Vorabentscheidungsverfahren in der Sache Blaise u.a. geht es zentral um die Vereinbarkeit der Verordnung (EG) 1107/2009 mit dem Vorsorgeprinzip. Das vorlegende französische Gericht möchte u.a. wissen, ob es mit dem Vorsorgeprinzip vereinbar ist, dass die zur Prüfung des Dossiers erforderlichen Tests, Analysen und Bewertungen von den Antragstellern durchgeführt würden, dass die Verordnung in keiner Weise eine Mehrzahl von Wirkstoffen und ihren kumulierten Einsatz berücksichtige und dass sie in ihren Kapiteln III und IV Pestizide in ihren Handelszusammensetzungen von Toxizitätsprüfungen (Genotoxizität, Karzinogenität, endokrine Schädlichkeit) ausnehme, indem sie lediglich summarische, stets vom Antragsteller durchgeführte Versuche verlange. ${ }^{406}$ Generalanwältin Sharpston wich in ihren Schlussanträgen im Fall Blaise u.a. den vorgelegten Fragen letztlich aus, indem sie darauf abstellte, dass das vorlegende Gericht die Verordnung (EG) Nr. 1107/2009 insgesamt infrage gestellt habe: Zum einen könnten Nichtigkeitsklagen nur wegen zu stark einschränkender, nicht jedoch wegen nicht hinreichend einschränkender Maßnahmen angegriffen werden. Zum anderen sei das Rechtsgebiet technisch und wissenschaftlich so komplex, dass ein entsprechend weiter Ermessensspielraum des Verordnungsgebers bestehe. $\mathrm{Da}$ die Verordnung unter Berücksichtigung dieses Spielraums jedoch weder offensichtlich ungeeignet noch sonst offensichtlich fehlerhaft sei, stehe ihre Gültigkeit nicht infrage. ${ }^{407}$

Aber auch gegen die Durchführungsverordnung (EU) 2017/2324 zur erneuten Genehmigung von Glyphosat sind etliche Klagen beim EuG eingereicht worden. Sie rügen u.a. die Verletzung des Vorsorgeprinzips, der Verpflichtung zur Gewährleistung eines hohen Gesundheits- und Umweltschutzniveaus und des Rechts auf Gesundheit. ${ }^{408}$ Allerdings hat das EuG teilweise schon entschieden, dass diese Individualklagen die Hürden des Art. 263 UAbs. 4 AEUV nicht überwinden. Es räumte zwar ein, dass mit der angegriffenen Durchführungsverordnung gewisse Aspekte wie die Erfüllung der Voraussetzungen von Art. 4 VO (EG) 1107/2009 geklärt seien. Das bedeute aber nicht, dass die Markt(wieder)zulassung der Anwendungsprodukte durch die Mitgliedstaaten automatisch erteilt werde. In jedem Falle könnten die Kläger vor nationalen Gerichten gegen diese Zulassungsakte vorgehen. Soweit in diesen Verfah-

406 Vorabentscheidungsersuchen des Tribunal correctionnel de Foix vom 26.10.2017, Rs. C-616/17, Procureur de la République/Matbien Blaise u. a., ECLI:EU:C:2019:190.

407 Schlussanträge der Generalanwältin Sharpston vom 12.03.2019, EuGH, Rs. C-616/17, Procureur de la République gegen Mathien Blaise et al., ECLI:EU:C:2019:190, S. auch o. E I. 2.

408 Klage vom 08.03.2018, Rs. T-178/18, Region Brüssel Hauptstadt/Kommission, ECLI:EU:T:2019:130. Zu den vom EuG schon entschiedenen Rechtssachen s. folgende Fn. 
ren eine europarechtliche Frage zu klären sei, helfe dann eine Vorlage an den EuGH (Art. 267 AEUV). ${ }^{409}$

Diese Entwicklung überzeugt nicht. Denn es geht betroffenen Individuen gerade um die Erfüllung der Voraussetzungen von Art. 4 VO (EG) 1107/2009. Hinzu kommt, dass die Notwendigkeit, gegen jede einzelne Zulassung einer unabsehbaren Anzahl glyphosathaltiger Anwendungsmittel vorzugehen, die Möglichkeiten Einzelner bei Weitem überschreitet. Insgesamt ist zu befürchten, dass die erneute Glyphosat-Wirkstoffgenehmigung schon ausgelaufen ist, bis Rechtsschutz gegen ihre Folgen im Bereich der Pflanzenschutzmittelzulassung erreicht sein dürfte. Es stellt sich deshalb auch die Frage, ob die Union in diesem Zusammenhang überhaupt noch effektiven Rechtsschutz gewährt (Art. 19 Abs. 1 UAbs. 2 EUV, Art. 47 GrCh, Art. 13 EMRK). Die vorgelegten Verbesserungsvorschläge für den Bereich der Pestizidzulassung, z.B. der Europäischen Ombudsfrau, ${ }^{410}$ können diesen Mangel nicht beheben.

\section{F. Ergebnisse}

\section{Mängel des Systems}

Von den zahlreichen Problempunkten und Mängeln, die der Beitrag identifiziert, sollen hier die folgenden hervorgehoben werden:

1. Es besteht ein erstaunliches Maß an Unklarheit, soweit es die tatsächlich abgesetzten Glyphosatmengen oder die Orte der Nutzung betrifft. Tonnenangaben für Glyphosat werden nicht separat ausgewiesen, sondern mit anderen Stoffen in der Wirkstoffgruppe „Organophosphor-Herbizide“ vermengt. Die Datenlage ist intransparent und unzureichend. [A II.1.]

2. Es gibt Anzeichen dafür, dass sich Glyphosatrückstände in zunehmend mehr Lebensmitteln wie auch im Trinkwasser finden und akkumulieren. Ferner zeigen sich Umweltschäden, insbesondere im Bereich der Biodiversität (u.a. Insektensterben). Das Grenzwertesystem für unbedenkliche Glyphosat- und AMPA-Mengen ist, wie widersprüchliche Festsetzungen, handelsinduzierte Hochsetzungen der Werte und ständige Änderungen zeigen, weder wissenschaftlich fundiert noch in sich konsistent. Es bilden sich Monopolstrukturen auf den Märkten für Pestizide und Saatgut, die zunehmend auch in die Entwicklungszusammenarbeit drängen. [A II. 2., C I.3.b)bb)]

3. Die Gesundheitsrisiken für Menschen sind i.E. hochumstritten. Durch Studien belegt ist aber, dass Glyphosat selbst in Städten im menschlichen Körper u.a. auch von Kita-Kindern gefunden werden kann. Während die Internationale Agentur

409 EuG, Rs.T-125/18, Associazione GranoSalus/Kommission, ECLI:EU:T:2019:92. Siehe auch EuG, Rs. T-12/17, Mellifera e.V., Vereinigung für wesensgemäße Bienenhaltung/ Kommission, ECLI:EU:T:2018:616 (zu Durchführungsverordnung [EU] 2016/1056); sowie EuG, T-600/15, Pesticide Action Network (PAN) u.a./Kommission, ECLI:EU:T: 2016:601, Rn. 33 (Genehmigung des Wirkstoffs Sulfoxaflor). In allen Fällen wurden die Nichtigkeitsklagen für unzulässig erklärt.

410 Fn. 208. 
für Krebsforschung (IARC) Glyphosat als „wahrscheinlich krebserregend“ der Kategorie 2A einstufte, gilt es nach europäischer Bewertung als „wahrscheinlich nicht krebserregend“, zumindest bei bestimmungsgemäßem Gebrauch. [A II.2., C I.3.a)]

4. In der Europäischen Union herrscht im Bereich der Zulassung und Anwendung von Herbiziden und Pestiziden eine komplexe Zuständigkeitsverteilung zwischen EU und Mitgliedstaaten, wobei die Wirkstoffgenehmigung auf der europäischen, die Pflanzenschutzmittelzulassung auf der mitgliedstaatlichen Ebene angesiedelt ist. Zugleich gibt es Spillover-Effekte in einzelnen Bereichen (Prüfung eines repräsentativen Anwendungsprodukts auf europäischer Ebene, Bindung der staatlichen Entscheidung an europäische Vorgaben). Insgesamt führt dies zur „Verantwortungsdiffusion“. Hinzu kommt, dass Wirkstoffgenehmigung und Pestizidzulassung exekutivlastige Bereiche darstellen, in denen die Rechte des Europäischen Parlaments schwach sind und es an einer Interessenvertretung für die Allgemeinheit und die Umwelt fehlt. [B, C, F II.]

5. Das Verfahren der Wirkstoffgenehmigung und Pestizidzulassung leidet an einem strukturellen Mangel, weil den antragstellenden Unternehmen ein maßgeblicher Einfluss auf die wissenschaftlichen Studien eingeräumt wird, die der behördlichen Bewertung zugrunde liegen. Sind die Behörden zu schwach ausgestattet oder sonst beeinflussbar, führt diese Konzeption des Verfahrens dazu, dass die Antragsteller letztlich selbst die Unbedenklichkeit ihrer Wirkstoffe und Produkte bestätigen. [C I.1.]

6. Es stellt ein Problem dar, dass das antragstellende Unternehmen das repräsentative Anwendungsprodukt, anhand dessen die Erfüllung der Anforderungen an die Sicherheit bewertet wird, selbst auswählen kann. Gegenstand der Bewertung ist damit nicht das Pflanzenschutzmittel, das in der jeweiligen Klimazone am meisten gebraucht wird, sondern dasjenige, das nach Ansicht des Antragstellers die besten Aussichten hat, zur gewünschten Genehmigung oder Zulassung zu führen. [C I. 2.b)]

7. Der Grundsatz der Transparenz gilt abstrakt und theoretisch, wird aber praktisch unterlaufen. Im Falle von Glyphosat wurden zwar über 6.000 Seiten Material öffentlich zugänglich gemacht. ${ }^{411}$ Wesentliche Grundlagen der Bewertung - insbesondere die Originalstudien der Unternehmen - unterfielen jedoch dem Schutz von Geschäftsgeheimnissen. [C I.2.c)]

8. Kennzeichnend für die Risikobewertung durch BfR und EFSA ist, dass Erkenntnisse über potentiell schädliche Auswirkungen als statistisch nicht signifikant oder sonst irrelevant abgetan werden, ohne dass das statistische Defizit aber nachvollziehbar verdeutlicht wird. Auch Mängel in der Darstellungsform von Studien, die z.B. einen Zusammenhang des Glyphosatgebrauchs mit dem Auftreten des NonHodgkin-Lymphoms belegen, werden herangezogen, um das inhaltliche Ergebnis in Zweifel zu ziehen. Welche anderen Faktoren die statistisch belegte Korrelation zwischen erhöhten Krebsraten und der Glyphosatexposition erklären könnten,

411 Mitteilung der Kommission vom 12.12.2017, C(2017) 8414 final, S. 11. 
bleibt offen. Das Ergebnis, wonach kein Krebsrisiko für Menschen „zu erwarten ist“, wird zudem unter den Vorbehalt der bestimmungsgemäßen Anwendung von Glyphosat gestellt, ohne dass thematisiert wird, wie realistisch eine solche Anwendung in den einzelnen Staaten ist. Die EFSA-Schlussfolgerung enthält bezüglich des Bodenmonitoring nur den Eintrag "no data". Trotz lückenhafter Datenlage ist zu erkennen, dass Glyphosat - wenn auch in noch geringen Mengen - die Natur einschließlich des Grundwassers erreicht hat und zunehmend durchdringt. [C I.3.a)]

9. Die Europäische Kommission hält trotz Hinweisen auf Risiken die Sicherheitsanforderungen für die erneute Glyphosatzulassung für erfüllt. Die Diskrepanz zur Bewertung des IARC (Krebsgefahr der Kategorie 2A) erklärt sie damit, dass sich das europäische Genehmigungsverfahren auf den Wirkstoff beschränke und mehr einschlägige Unterlagen berücksichtigen konnte, namentlich auch „nicht öffentlich zugängliche" Studien. [C I.3.a)]

10. Die Durchführungsverordnung zur Wiedergenehmigung von Glyphosat lässt nicht klar erkennen, welche Auflagen und Einschränkungen für die Glyphosatnutzung nun eigentlich gelten, sondern verweist stattdessen auf ein Dickicht anderer Regelungen und Grundsätze. Damit wird für die Öffentlichkeit, die an dieser Stelle auf eine transparente Darstellung angewiesen ist, der Zugriff auf wesentliche Informationen erschwert. Die vermeintlichen Einschränkungen, die die Durchführungsverordnung zur Wiedergenehmigung von Glyphosat enthalten soll, entpuppen sich bei genauerem Hinsehen sämtlich als allgemein gehaltene Appelle an die Mitgliedstaaten, gewissen Aspekten bei der Pflanzenschutzmittel-Neuzulassung besondere Aufmerksamkeit zu schenken und auf bestimmte Belange zu „achten“. Die Europäische Kommission verschiebt damit einen Großteil ihrer Verantwortung auf die Mitgliedstaaten. [C I.3.b) aa)]

11. Die Kommission erwähnt in der Durchführungsverordnung zur Wiedergenehmigung von Glyphosat als critical area of concern den Umstand, dass im Glyphosat-Wiedergenehmigungsverfahren acht der 24 antragstellenden Unternehmen Spezifikationen präsentiert hatten, die nicht durch die toxikologische Bewertung gestützt waren. Statt jedoch zu klären, ob sich diese Verfälschungen auf das Bewertungsergebnis im Wirkstoff-Neugenehmigungsverfahren ausgewirkt haben könnten, verschiebt sie auch diese Angelegenheit in die PflanzenschutzmittelNeuzulassung, d.h. zu den Mitgliedstaaten. [C I.3.b) aa)]

12. Die Höchstrückstandswerte (Maximum Residue Levels [MRLs]) für Glyphosat, die im Anschluss an die Wiedergenehmigung festgesetzt werden müssen, schwanken für die einzelnen Lebensmittel immer wieder erheblich, zum Teil auch in Abhängigkeit von Außenhandelsbeziehungen, und beziehen teilweise relevante Forschungsdaten nicht ein. Man muss deshalb zu dem Schluss kommen, dass die Werte einen eher gewillkürten als wissenschaftlichen Charakter haben. [C I.3.b) bb)]

13. Die Behauptung des deutschen Landwirtschaftsministers, er habe nur durch seine Zustimmung erreichen können, den Glyphosat-Einsatz „für privaten Gebrauch und für andere Gebräuche zu reduzieren“, ist irreführend. Zwar hätte die Kommission die Entscheidung selbst treffen können, wenn der Berufungsausschuss 
SCoPAFF keine Mehrheit für eine Stellungnahme erreicht hätte. Dann hätte sie auch nur den vorgelegten Entwurf einer Durchführungsverordnung zur Wiedergenehmigung annehmen können. Die durch Deutschland im ACoPAFF erreichten Zusatzklauseln beinhalten aber insbesondere in puncto Amateurverwendung keinen Mehrwert gegenüber der vorhandenen Rechtslage. [C I.3.c)]

14. In der sogenannten „Portier-Kontroverse“ traten fast $100 \mathrm{~W}$ issenschaftler und Wissenschaftlerinnen aus aller Welt der Bewertung von EFSA und des deutschen BfR offen entgegen. Die Einschätzung der IARC sei „bei Weitem glaubwürdiger“ als die der europäischen Institutionen, weil sie anders als diese auf einem offenen und transparenten Verfahren beruhe. Portier zeigte auf, dass Daten zurückgehalten und tumorrelevante Studien nicht berücksichtigt worden waren. EFSA versuchte die Diskrepanzen mit einem abweichenden methodischen Ansatz zu erklären. Dies führte im Ergebnis dazu, dass unbestreitbare statistische Signifikanzen mithilfe eines gewichtenden Ansatzes neutralisiert wurden. [C I.4.a)]

15. Nach Enthüllung der sogenannten „Monsanto-Papers“ steht die Unabhängigkeit der entscheidenden Institutionen infrage. Es ist den glyphosatherstellenden Unternehmen gelungen, sich tief in den Entscheidungsstrukturen zu verankern und Einfluss auf die maßgeblichen „Stakeholder“ im Genehmigungsprozess zu nehmen. Davon ist insbesondere auch das deutsche BfR betroffen. Es gab kontinuierliche Kontakte und Auftragsbeziehungen zwischen der Glyphosate Task Force (Antragstellerin) bzw. ihr nahestehenden Firmen und den deutschen Genehmigungsbehörden. Es konnte nachgewiesen werden, dass erheblich große und relevante Teile aus dem Renewal Assessment Report des BfR, die auch in den Schlussbericht von EFSA eingingen, wortwörtlich mit einem Monsanto-Papier übereinstimmten. Dabei handelt es sich um Plagiate, weil die betroffenen Textstellen nicht die wahre Quelle offenlegen. Die internationale Presse hat anhand der Auswertung der „Monsanto-Papers“ aufgezeigt, dass Monsanto systematisch Wissenschaftler und Wissenschaftlerinnen kontaktierte, damit diese positive Studien über Glyphosat veröffentlichten, und dafür wohl auch bezahlte. Zugleich wurden Strategien aufgedeckt, Wissenschaftler und Wissenschaftlerinnen zu diskreditieren, die sich kritisch zu Monsanto-Produkten äußerten. Das Ausmaß der Funde ist insgesamt so erheblich, dass von einer unabhängig geführten Bewertung insbesondere der Gesundheitsrisiken keine Rede sein kann und Auswirkungen auf das Ergebnis nicht ausgeschlossen werden können. [C I.4.]

16. Für die Zulassung von Pflanzenschutzmitteln sind die Mitgliedstaaten zuständig, wobei ein zonales Bewertungsverfahren stattfindet, das die Bewertung für alle EULänder derselben geografischen Zone bei einem Mitgliedstaat konzentriert. Der Mitgliedstaat soll zwar über ein beträchtliches Ermessen bei seiner Entscheidung über die Zulassung in seinem Staatsgebiet verfügen, darf aber weder die zonale Bewertung noch die vorgängige Wirkstoffgenehmigung unterlaufen. Gemäß Art. 36 Abs. 3 bzw. Art. 31 VO (EG) 1107/2009 müssen Einschränkungen der Zulassung vielmehr durch die spezifischen ökologischen oder landwirtschaftlichen Verhältnisse des betreffenden Landes gerechtfertigt sein. Dasselbe gilt für die 
obligatorische Erneuerung der Zulassung von Pflanzenschutzmitteln im Falle der Neugenehmigung des Wirkstoffs. [C II.1.b) und c)]

17. Eineinhalb Jahre nach der Neugenehmigung von Glyphosat hat der deutsche Gesetz- bzw. Verordnungsgeber weder die Beschränkungen aufgegriffen, die die europäische Durchführungsverordnung zur erneuten Genehmigung von Glyphosat aufträgt, noch eigene Ankündigungen zur Einschränkung des Gebrauchs umgesetzt. Die angekündigte Novelle der Pflanzenschutz-Anwendungsverordnung befindet sich immer noch in der Ressortabstimmung der Bundesregierung. [C II.2.]

18. Soweit es die erforderliche Neuzulassung aller glyphosathaltigen Pflanzenschutzmittel in Deutschland betrifft, ist die Lage zum Teil undurchsichtig und durch Meinungsverschiedenheiten zwischen den beteiligten Bundesministerien bzw. -behörden gekennzeichnet. Das letztentscheidende BVL beruft sich zunehmend auf Verzögerungen des Verfahrensablaufs, die nicht die Antragsteller zu verantworten hätten, um bestehende Zulassungen gemäß Art. 43 Abs. 6 VO (EG) 1107/2009 um zunächst ein Jahr zu verlängern. Aufgrund dieser problematisch konzipierten Vorschrift wird es möglich, alle Vorgaben aus der schon geltenden neuen Glyphosat-Wirkstoffgenehmigung zum Schutz von Umwelt und Gesundheit vorerst leerlaufen lassen. Soweit die Zulassungsdauer bei einzelnen Pflanzenschutzmitteln auch ohne Verlängerung schon über 2018 hinausläuft, erscheint fraglich, ob die Anforderungen der deutschen und vor allem europäischen Überleitungsvorschriften dafür eine Rechtfertigung bieten. [C II.3.a)]

19. Soweit es die in einzelnen Mitgliedstaaten umgesetzten bzw. in der Diskussion befindlichen Einschränkungen des Glyphosat-Gebrauchs betrifft, sind diese angesichts des geltenden Rechts nicht leicht zu realisieren. Obwohl die Durchführungsverordnung zur Neugenehmigung von Glyphosat beinhalten soll, die Verwendung durch Amateure, zur Sikkation und auf öffentlichen Flächen einzudämmen wie auch die Biodiversität zu schützen, bleibt sie in den entscheidenden Punkten so vage, dass Reduktions- oder Ausstiegsstrategien nur unter Beachtung der Voraussetzungen der Art. 36 Abs. 3 bzw. Art. 31 VO (EG) 1107/2009 angewandt werden können. Danach müssen Einschränkungen der Zulassung durch die spezifische ökologischen oder landwirtschaftlichen Verhältnisse des betreffenden Landes gerechtfertigt sein und dürfen vorgängige Bewertungsprozesse nicht desavouieren. Zwar können Art. 1 Abs. 4 Satz 2 VO (EG) 1107/2009 (Vorsorgeprinzip) sowie Art. 1 Abs. 3 Verordnung (EG) 1107/2009 (Gewährleistung eines „hohen Schutzniveaus für die Gesundheit von Mensch und Tier und für die Umwelt") die Handlungsspielräume der Mitgliedstaaten erweitern. Sie bieten aber keine eigenständige Ermächtigungsgrundlage für Einschränkungen. Als zusätzlich kontraproduktiv erweisen sich die von der europäischen Pflanzenschutzmittelverordnung in Bezug genommenen „einheitlichen Grundsätze“ i.S.v. Art. 29 Abs. 6 VO (EG) 1107/2009, die teilweise hohe zusätzliche Anforderungen an Beschränkungen stellen und generell von der Tendenz geprägt sind, die Pestizidverwendung so wenig wie möglich einzuschränken. Setzt die Durchführungsverordnung zur Wirkstoff-Neugenehmigung - wie im Glyphosat-Fall - nicht selbst klare Vorgaben, sondern lässt stattdessen die Grundsätze zum Zuge kommen oder ver- 
weist sogar noch ausdrücklich auf sie, werden die vermeintlichen Sicherheitsanforderungen der Durchführungsverordnung noch einmal deutlich abgeschwächt, ohne dass man ihnen das ansieht. [C II.3.c)]

20. Auch nach der Genehmigung des Wirkstoffs kann die Kommission die Genehmigung eines Wirkstoffes jederzeit überprüfen und eine Aufhebungs- oder Änderungsverordnung erlassen, wenn die Genehmigungsvoraussetzungen nicht mehr vorliegen (Art. 21 VO [EG] 1107/2009). Entsprechend haben die Mitgliedstaaten die Möglichkeit, eine Pflanzenschutzmittel-Zulassung nachträglich zu beschränken oder aufzuheben (Art. 44 VO [EG] 1107/2009. Ferner können Notfallmaßnahmen getroffen werden, um Wirkstoffe oder Pflanzenschutzmittel unverzüglich aus dem Verkehr zu ziehen (Art. 69 ff. VO [EG] 1107/2009). Da jedoch kein Monitoring-System existiert, das die Langzeiteffekte der auf den Markt gebrachten Pestizide überwacht, greifen die Regelungen erst dann, wenn sich die Schäden von selbst zeigen, also schon eingetreten sind. [D I.1.-3.]

21. Art. 4 der Richtlinie 2009/128/EG über einen Aktionsrahmen der Gemeinschaft für die nachhaltige Verwendung von Pestiziden (Pestizid-Rahmenrichtlinie) verpflichtet die Mitgliedstaaten, Nationale Aktionspläne zur nachhaltigen Anwendung von Pflanzenschutzmitteln zu verabschieden. Diese Richtlinie wird nicht nur von etlichen Mitgliedstaaten ignoriert, ohne dass die Kommission dagegen einschreitet. Ihre Zwecksetzung findet auch keine Berücksichtigung bei der Wirkstoffgenehmigung bzw. Pflanzenschutzmittel-Zulassung, so dass es im Verhältnis zwischen der Pestizid-Rahmenrichtlinie 2009/128/EG und der Pflanzenschutzmittelverordnung (EG) 1107/2009 an Konsistenz fehlt. [D I.4.]

22. Die Pestizidstatistik-Verordnung (EG) $1185 / 2009$ schafft einen gemeinsamen Rahmen für die systematische Erstellung europäischer Statistiken über das Inverkehrbringen und die Verwendung von Pestiziden. Zwar übermitteln die Mitgliedstaaten genaue Daten an die Kommission, die sie grundsätzlich auch der Öffentlichkeit zur Verfügung stellen muss. Allerdings bestimmt Art. 3 Abs. 4 VO 1185/2009, dass Eurostat die Daten „aus Gründen der Vertraulichkeit“ nach chemischen Produktklassen oder -kategorien aggregiert, so dass die Öffentlichkeit nur Gesamtdaten zu „Organophosphor-Herbiziden“ erhält und deshalb nicht erfährt, wieviel Glyphosat in den einzelnen Mitgliedstaaten in den Verkehr gebracht und für die dort repräsentativen Kulturpflanzen verwendet werden. Das Ergebnis dieser Publikationspraxis ist ein hohes Maß an Intransparenz, das eine wirksame Kontrolle durch Presse und Öffentlichkeit massiv erschwert. [D I.4.]

23. Das deutsche Recht (PflSchG) sieht den Widerruf und die Rücknahme von Pflanzenschutzmittel-Zulassungen vor. Sind z.B. die Anforderungen des Art. 29 VO (EG) 1107/2009 nicht mehr erfüllt, könnte Deutschland gemäß Art. 44 Abs. 3 VO (EG) 1107/2009 verpflichtet sein, die Zulassung aufzuheben. Nach deutschem Recht kann dies aber nur binnen eines Jahres nach Kenntnis der Behörde von den Tatsachen geschehen, so dass die Möglichkeit besteht, eine Zulassung durch Verstreichenlassen der Frist zu erhalten. Wird eine Zulassungen aufgehoben, weil z.B. aufgrund neuer wissenschaftlicher Erkenntnisse klar wird, dass die Anforderungen des Art. 29 VO (EG) 1107/2009 von Anfang an nicht erfüllt waren oder nicht 
mehr erfüllt sind, kommt Entschädigung in Betracht. Diese ist aber nur zu gewähren, „soweit dies zur Abwendung oder zum Ausgleich unbilliger Härten geboten erscheint". ( $\$ 54$ Abs. 2 PflSchG). Es erscheint nicht als unbillige Härte, wenn Unternehmen, insbesondere solche, die an der "Glyphosate Task Force“ beteiligt waren, nach monatelangen Kämpfen um die Bewertung des Risikos keine Entschädigung erhalten. Denn sie mussten von vornherein damit rechnen, dass sich die Risiken im Zusammenhang mit diesem Wirkstoff und seinen Anwendungsprodukten im Laufe der Zeit erhärten oder besser nachgewiesen werden könnten. [D II.1.]

24. Gemäß dem Auftrag aus Art. 4 der Pestizid-Rahmenrichtlinie 2009/128/EG und in Übereinstimmung mit $\$ 4$ PflSchG hat die Bundesregierung im April 2013 den „Nationalen Aktionsplan zur nachhaltigen Anwendung von Pflanzenschutzmitteln" erstellt. In diesem Zusammenhang wurde das Pflanzenschutzgesetz an etlichen Stellen nachgebessert. Es ist allerdings nicht erkennbar, wie die Vorgabe des Art. 12 Pestizid-Rahmenrichtlinie (Maßnahmen zur Verringerung des Einsatzes von Pflanzenschutzmitteln auf öffentlichen Flächen, Schutzgebieten etc.) erfüllt werden soll. Die Datenerhebung, wie sie die europäische Pestizidstatistik-Verordnung (EG) 1185/2009 vorschreibt, ist Teil des deutschen Aktionsplans. Das „Wirkstoffranking“, wie es das Julius-Kühn-Institut im Rahmen der jährlichen „PAPA-Erhebungen“ durchführt, ist aber weitgehend unbrauchbar, weil mit Bezug auf die in Deutschland wichtigsten Nutzpflanzen nur Schätzwerte für Gesamtdeutschland präsentiert werden, die das JKI selbst infolge einer nur geringen Stichprobengröße für unsicher hält. So kann die Öffentlichkeit nicht erfahren, in welchen Regionen Deutschlands welche relevanten Kulturpflanzen besonders stark oder wenig mit Glyphosat behandelt sind. [D II.2.]

25. Um die Handhabung der Kontrollaufgaben in ganz Deutschland zu harmonisieren, haben Bund und Länder ein gemeinsames „Pflanzenschutz-Kontrollprogramm" installiert. Das Kontrollprogramm überzeugt insoweit, als es aufzeigt, wie viele Betriebe tatsächlich kontrolliert wurden und wie hoch die Beanstandungsquote in den einzelnen Bereichen ist. Andererseits vermittelt der Jahresbericht nicht den Eindruck, als habe man wirklich wissen wollen, warum Beanstandungsquoten ungeachtet des aufwändigen Kontrollprogramms gestiegen und nicht gesunken sind. Die allzu schnell gegebenen pauschalen Erklärungen für festgestellte Abweichungen stellen sich wie Rechtfertigungsversuche dafür dar, es beim Status quo (d.h. Kontrolle in unverändertem Ausmaß mit gelegentlichen Kontrollschwerpunkten) zu belassen. [D II.3.]

26. Unter den hier gegebenen Umständen - schnelles Voranschreiten der wissenschaftlichen Entwicklung speziell bei Glyphosat (Erwägungsgrund $19 \mathrm{f}$. DurchführungsVO zur Wiedergenehmigung), Existenz einer ernstzunehmenden Mindermeinung, Annahme eines Risikos durch die maßgebliche WHO-Institution, statistische Belegefür ein Risiko, inihrer Relevanz bestritteneKohortenstudien mit Annahmeeines Risikos,Ergebnissegerichtlicher BeweisaufnahmenindenUSA,fehlendeUnabhängigkeit der Bewertung bei gleichzeitiger Geheimhaltung maßgeblicher Studien, die ein Risiko als „unwahrscheinlich“ einstufen-ist dieMöglichkeit eines Gesundheits- 
risikos alles Andere als auszuschließen. Infolgedessen hätte das Vorsorgeprinzip mindestens verlangt, effektiv risikomindernde Vorgaben zum Gesundheitsschutzin die DurchführungsVO zur Wiedergenehmigung von Glyphosat aufzunehmen. Soweit es die Umweltrisiken betrifft, fehlt es an einer nachvollziehbaren Behandlung der Frage, ob diefestgestellten schädlichen Auswirkungen insbesondere auf die Biodiversität „unannehmbar" im Sinne des europäischen Rechts sind. Es fehlt insoweit bereits an Kriterien. Das Vorsorgeprinzip wurde verletzt, weil ungeachtet von Anhaltspunkten für mögliche Gesundheitsrisiken und festgestellten Umweltrisiken keine ernstzunehmenden Vorgabenzur Risikominderung in dieDurchführungsVO für Glyphosat aufgenommen wurden, sondern die Verantwortung stattdessen umfassend an die Mitgliedstaaten delegiert wurde, ohnedass dieseaber beider folgenden Zulassung der Anwendungsmittel die Wirkstoffgenehmigung noch grundlegend einschränken könnten. Zudem hätte das Vorsorgeprinzip eine Wiederholung der nicht unabhängig und objektiv durchgeführten Bewertung in angemessener Form verlangt. [E I.]

27. Art. 6 Abs. 1 Århus-Verordnung bzw. Art. 4 Abs. 2 Århus-Richtlinie gewähren Individuen und Verbänden ein Recht auf Zugang zu „Informationen über Emissionen in die Umwelt“. Art. 63 Abs. 3 VO 1107/2009 (EG) lässt die Umweltinformationsrechte ausdrücklich unberührt. Unter den Emissionsbegriff fällt nach europäischer Rechtsprechung auch das Freisetzen von Stoffen oder Produkten wie z.B. Pflanzenschutzmitteln. Das Zugangsrecht erfasst auch den Zugang zu wissenschaftlichen Studien, insbesondere zur Toxizität. Daran gemessen, ist der Standpunkt der Kommission, wonach die europäischen Behörden im GlyphosatGenehmigungsverfahren über qualitativ überlegene Studien verfügt hätten, die zwar maßgeblich die Unwahrscheinlichkeit von Risiken belegten, aber im Interesse des Schutzes des geistigen Eigentums der Unternehmen geheim bleiben müssten, unhaltbar. Die Reformvorschläge der Kommission zur Steigerung der Transparenz dürften die Lage nicht grundlegend verbessern, weil sie dem herkömmlichen Abwägungsansatz „Transparenz versus Betriebsgeheimnisse“ verhaftet bleiben. [E II.1.]

28. Das Vorsorgeprinzip (Art. 191 Abs. 2 UAbs. 1 Satz 2 AEUV, Art. 1 Abs. 4 VO 1107/2009) und die Pflicht zur Sicherstellung eines ",hohen Gesundheitsniveaus“" (Art. 35 Satz 2 GrCh i.V.m. Art. 9, Art. 168 Abs. 1 UAbs. 1 AEUV, Art. 1 Abs. 3 VO 1107/2009) sind Leitprinzipien der Interpretation umwelt- und gesundheitsrelevanter europäischer Grundrechte. Aus ihnen können zwar weder konkrete Verpflichtungen zum Ergreifen bestimmter Maßnahmen noch subjektive Rechte abgeleitet werden. Als verbindliche Zielvorgaben werden sie aber verletzt, wenn ihnen entgegengehandelt wird oder sie ignoriert werden. Zu beachten ist, dass das hohe Gesundheitsniveau „sichergestellt“ und nicht lediglich angestrebt werden muss. [E II.2.a)]

29. Art. 35 Satz $1 \mathrm{GrCh}$ vermittelt ein europäisches Grundrecht auf Zugang zur Gesundheitsvorsorge. Dies umfasst in Anlehnung an Art. 11 Abs. 3 EuSozialCh präventive Maßnahmen, die Krankheiten verhindern oder (schon eingetretene) begrenzen. Maßnahmen zum Schutz der Umwelt sind erforderlich, wenn 
Umweltverschmutzungen zu Krankheiten führen können. Ungeachtet ihres weiten Gestaltungsspielraums sind die Staaten auch bei komplexen Umweltproblemen verpflichtet, alle Anstrengungen zu unternehmen, um das Ziel innerhalb vernünftiger Zeit $\mathrm{zu}$ erreichen. Dazu gehören ein angemessener Kontrollmechanismus und nötigenfalls vorsorgliche Maßnahmen, um wie im Falle von Glyphosat insbesondere Erkrankungen am Non-Hodgkin-Syndrom zu verhindern. Die festgesetzten Höchstmengen für Rückstände in Lebensmitteln und Trinkwasser müssen nicht nur dem Vorsorgeprinzip, sondern auch dem Ziel, ein „hohes Gesundheitsniveau“ sicherzustellen, entsprechen. Unvereinbar damit ist die Aussage, dass „ein wenig Glyphosat im Körper“ in einer hochtechnisierten Gesellschaft unvermeidlich, sozialadäquat und aus übergeordneten Gründen hinzunehmen sei. Das gilt jedenfalls dann, wenn wie im Falle von Glyphosat oder glyphosathaltiger Mittel die Exposition nur "wahrscheinlich nicht kanzerogen“ ist, die Rückstandshöchstmengen umstritten oder unsicher sind und auch die Unabhängigkeit der Bewertung erheblichen Zweifeln unterliegt [E II.2.b)]

30. Gemäß Art. $7 \mathrm{GrCh}$, der im Zweifel wie Art. 8 EMRK zu interpretieren ist (Art. 52 Abs. 3 GrCh), können schwerwiegende Umweltbelastungen oder das Verschweigen relevanter Umweltinformationen das Recht auf den ungestörten Genuss der Privat- und Wohnsphäre verletzen, und zwar selbst dann, wenn keine schwerwiegende Beeinträchtigung der Gesundheit droht bzw. dies umstritten ist. Obwohl auch hier ein weiter Gestaltungsspielraum bei der Abwägung zwischen Umwelt- und widerstreitenden Belangen besteht, verengt sich dieser stark, wenn es um „intime Aspekte“ des Privatlebens geht. Das Einbringen von Pestiziden in den menschlichen Körpers ist eine besonders intrusive Art der Inanspruchnahme, die generell als schwerwiegend gelten muss. Die Einhaltung von regulär festgelegten Grenzwerten und Höchstmengen kann dem nicht entgegengehalten werden, wenn die Grenzwerte fragwürdig zustande gekommen, die Abklärungen unvollständig gewesen sind, die Grenzwerte erheblich schwanken oder das Vorsorgeprinzip verletzt wurde. [E II.2.c)]

31. Nach hier vertretener Auffassung beinhaltet das Recht auf Privatsphäre auch das Recht auf einen pestizidfreien Körper und Schutz vor Kontamination (Art. 3, 7 GrCh, Art. 8 EMRK). Selbst wenn die Dosis minimal wäre, Glyphosat zu 100\% wieder aus dem menschlichen Körper ausgeschieden und keine Gesundheitsschädigung (Art. $3 \mathrm{GrCh}$ ) ausgelöst würde, kann die intrusive Form der Inanspruchnahme die Selbstbestimmung über den eigenen Körper und damit das Recht auf Schutz der Privatsphäre (Art. 7 GrCh, Art. 8 EMRK) beeinträchtigen. Anknüpfungspunkt ist, dass das Individuum Stoffe in seinen Körper hineinlassen muss, die dort nichts zu suchen haben. Es besteht Integritätsschutz vor auch nur möglicherweise riskanten Stoffen im Sinne eines Selbstbestimmungsrechts über den eigenen Körper - „habeas corpus“ in seiner eigentlichsten und innerlichsten physischen Form. Wiederum gilt: Die Abwägung mit kollidierenden Interessen und Rechtsgütern wird durch die Einhaltung von regulär festgelegten Grenzwerten und Höchstmengen nur dann vorweggenommen, wenn diese wissenschaftlich abgesichert sind, keinem ständigen Wandel unterliegen, die ihnen zugrunde liegen- 
den Abklärungen vollständig waren und das Vorsorgeprinzip beachtet wurde. [E II.2.d)]

32. Klagen im Zusammenhang mit Glyphosat gelangen zunehmend vor die europäischen Gerichte. Im der Sache Blaise steht die Vereinbarkeit der Verordnung (EG) 1107/2009 mit Europarecht, insbesondere auch dem Vorsorgeprinzip, auf dem Prüfstand. Nach Auffassung der Generalanwältin leidet die Verordnung jedoch nicht an so offensichtlichen Mängeln, dass ihre Gültigkeit dadurch infrage gestellt würde. Auch Klagen gegen die Durchführungsverordnung (EU) 2017/2324 zur erneuten Genehmigung von Glyphosat u.a. wegen Verletzung des Vorsorgeprinzips, der Verpflichtung zur Gewährleistung eines hohen Gesundheits- und Umweltschutzniveaus und des Rechts auf Gesundheit sind wenig aussichtsreich. Die betreffenden Individualklagen, die das EuG schon entschieden hat, konnten die Hürden des Art. 263 UAbs. 4 AEUV nicht überwinden. Weil die Anwendungsprodukte durch die Mitgliedstaaten nicht automatisch aufgrund der Wiedergenehmigung von Glyphosat wieder zum Markt zugelassen würden, müssen die Kläger vor nationalen Gerichten gegen jede einzelne Zulassung vorgehen, die bis zum Auslaufen der aktuellen Wiedergenehmigung von Glyphosat aber wohl kaum entschieden haben dürften. Es stellt sich deshalb die Frage, ob die Union in diesem Kontext noch effektiven Rechtsschutz gewährt (Art. 19 Abs. 1 UAbs. 2 EUV, Art. 47 GrCh, Art. 13 EMRK). [E III.]

\section{Bewertung der Situation und Ausblick auf Neues auf dem „Shikimate pathway“}

Die Sicherheit von Pestiziden kann im Rahmen des vorhandenen Systems der Zusammenarbeit von EU und Mitgliedstaaten auf Basis der Pflanzenschutzmittelverordnung (EG) 1107/2009 nicht gewährleistet werden. Wie das Beispiel der erneuten Genehmigung von Glyphosat zeigt, entziehen sich die verantwortlichen Stellen einer wirksamen Kontrolle. Der Kern des Problems liegt darin, dass die Wirkstoffgenehmigung auf der europäischen Ebene, die Zulassung der Anwendungsprodukte hingegen bei den Mitgliedstaaten angesiedelt ist. Es kommt dadurch zu sich gegenseitig neutralisierenden Prozessen: Einerseits besteht für die EU ein Anreiz, im Verfahren der Wirkstoffgenehmigung von klaren risikomindernden Vorgaben abzusehen und stattdessen die einzelnen Mitgliedstaaten in die Pflicht zu nehmen, Bedenken im späteren Pflanzenschutzmittel-Zulassungsverfahren Rechnung zu tragen. Treibend hierfür ist das Komitologieverfahren, das bei mangelnder Einigkeit innerhalb der Mitgliedstaaten zu Kompromissformeln und Ausweichverhalten auf Seiten der Kommission führt. Andererseits können die Mitgliedstaaten im Pflanzenschutzmittel-Zulassungsverfahren wiederum darauf verweisen, dass der zugrunde liegende Wirkstoff (z.B. Glyphosat) ja in einem aufwändigen europäischen Bewertungsverfahren genehmigt wurde. Das vorhandene europäische Recht stützt diese Position, indem es Neubewertungen des Wirkstoffs im Produktzulassungsverfahren an enge Voraussetzungen knüpft. Dieses Grundproblem setzt sich auch in den Bereich des Rechtsschutzes fort, indem Individualklagen gegen die Wirkstoffgenehmigung bis- 
lang als unzulässig abgewiesen werden, weil aus ihr noch nicht die Zulassung konkreter Anwendungsprodukte folge - mit der Konsequenz, dass betroffene Einzelne und Verbände gegen die Zulassung jedes einzelnen glyphosathaltigen Mittels in den verschiedenen Mitgliedstaaten vorgehen müssen. Wenn sie das tun, werden sie dann auf das Problem stoßen, dass die Erfüllung der zentralen Voraussetzungen von Art. 4 VO (EG) 1107/2009 betreffend den Gesundheits- und Umweltschutz im Wirkstoffgenehmigungsverfahren grundsätzlich geklärt wurde und allenfalls landesspezifische Besonderheiten noch zu Einschränkungen führen können. Inwieweit einzelne Mitgliedstaaten angesichts der erneuten Glyphosatgenehmigung Reduktionsstrategien wie z.B. ein völliges Verbot der Nutzung glyphosathaltiger Mittel durch Amateure oder zur Verwendung vor der Ernte zur sogenannten Sikkation einführen dürfen, ist fraglich, weil die Durchführungsverordnung zur erneuten Genehmigung von Glyphosat in allen entscheidenden Punkten vage bleibt.

Wir sehen im Bereich der Pestizidzulassung nicht nur ein System der organisierten Verantwortungsdiffusion, in dem sich die Verantwortung von EU und Mitgliedstaaten bis zur Unkenntlichkeit verdünnt und die einzelnen Mitgliedstaaten sich hinter den Ausschussmauern der Komitologie verbergen. Wir sehen auch das Phänomen der Überkomplexität, wie es nicht nur den Umgang mit Pestiziden betrifft. Glyphosathaltige Herbizide haben in ganz besonderem Ausmaß Milliardenumsätze kreiert, die sich in einer unüberbrückbaren Kluft zwischen extrem gut ausgestatteten Unternehmen und zunehmend ressourcenknappen und personalschwachen Kontrollbehörden niederschlagen. Der Umstand, dass das deutsche BfR seitenweise Monsanto-Texte ohne Angabe der Quelle in seine eigenen Bewertungsdokumente übernimmt und dies wiederum in die europäischen Bewertungsberichte gelangt, ist vor diesem Hintergrund zu sehen. Die Anhaltspunkte für eine viel zu starke Vernetzung der Kontrollbehörden mit den interessierten Unternehmen sind insgesamt, wie der Beitrag zeigt, so stark, dass das Vertrauen in eine objektive und transparente Überprüfung von Wirkstoffen und Pflanzenschutzmitteln schwerwiegend erschüttert ist.

Es gibt keine Patentlösung. Zwingend erforderlich ist eine Verbesserung der Datenlage und Transparenz über das schon geplante Maß hinaus. Die Verschleierung von Glyphosatmengen in Wirkstoffgruppen und dergleichen muss beendet werden. Der Schutz von Betriebsgeheimnissen muss weiter respektiert werden, darf aber grundsätzlich nicht dazu führen, dass wie im Glyphosatfall Studien, die das Ergebnis „Unbedenklichkeit" maßgeblich tragen, geheim gehalten werden. Der Unternehmenseinfluss auf die Verfahren (Auswahl des RMS, Bedeutung der Originalstudien etc.) ist zu groß. Besondere Aufmerksamkeit erfordert die Unabhängigkeit der Wissenschaft, die, obwohl auf ihr die Risikobewertung ruht, bei immer knapperer Ausstattung in immer größere Abhängigkeit von drittmittelstiftenden und sonst finanzierenden Unternehmen gerät. Möglicherweise könnte in schwierigen Fällen, in denen Risiken umstritten sind, ein System helfen, bei dem die prüfenden Wissenschaftler und Wissenschaftlerinnen unter denjenigen mit einschlägiger Expertise nach dem Zufallsprinzip ausgewählt und beauftragt werden. Nachzuschärfen ist vor allem auch der Umgang mit dem Vorsorgeprinzip. Eine derartige Ansammlung von risikoindizierenden Anhaltspunkten wie im Glyphosatfall muss effektive risikomindernde Auflagen zur Folge haben. 
An die Entwicklung entsprechender Kriterienkataloge ist zu denken - auch wenn dies die Überkomplexität nicht senken dürfte. Insgesamt muss Sorge dafür getragen werden, dass die Verantwortlichkeiten im Wirkstoffverfahren und im Produktzulassungsverfahren klar getrennt sind und tatsächlich wahrgenommen werden. Es muss darüber nachgedacht werden, wie die oft gegenläufig agierenden Kreise der Landwirtschaftsminister und -ministerinnen einerseits und der Umweltminister und -ministerinnen andererseits zusammengeführt werden können, um gerade auch auf der europäischen Ebene die Konsistenz zwischen beiden Regelungsgebieten und zugehöriger Praxis zu stärken. Die Umsetzung durch die Mitgliedstaaten muss einem Monitoring unterliegen. Vieles Weitere wie die Aktualisierung der einheitlichen Grundsätze und Leitlinien, die Offenlegung der Verhandlungen in den Komitologieausschüssen, die vollständige Integration der Rahmen-Richtlinie (2009/128/EG) in die neu auszuhandelnde Gemeinsame Agrarpolitik für die Zeit nach 2020, ein Verbot des Glyphosateinsatzes zur Sikkation, etc., ist in der Diskussion. ${ }^{412}$ Sicher erscheint nur: Das System bedarf grundlegender Reform.

Wie wird die Zukunft aussehen? Ein Forscherteam an der Universität Tübingen präsentierte Anfang 2019 einen seltenen Zucker namens 7-deoxy-Sedoheptulose (7dSh), der den sogenannten „Shikimate pathway“ bei Pflanzen blockiert und damit Glyphosat ersetzen können soll. ${ }^{413}$ Glyphosat blockiert ebenfalls die Enzyme des Shikimate pathway, wenn auch auf andere Weise. Auch beim neuen Metaboliten ist wieder die Rede davon, dass das blockierte Enzym weder bei Menschen noch bei Tieren vorkomme und an menschlichen Zelllinien keinerlei Zytotoxizität festgestellt werden konnte. Das Thema wird also aktuell bleiben. Alle nötigen Erfahrungen liegen vor.

\section{BIBLIOGRAPHIE}

AWATER-ESPER, STEFANIE, Glyphosat: So reagieren andere EU-Mitgliedstaaten, 04.12.2017, abrufbar unter: https://www.topagrar.com/acker/news/glyphosa t-so-reagieren-andere-eu-mitgliedstaaten-9850032.html (15.06.2019)

BENBROOK, CHARLES M., Trends in glyphosate herbicide use in the United States and globally, Environmental Sciences Europe, 2016, Vol. 28, S. 1

BORWIECK, KAROLINE, Chemischer Pflanzenschutz und Biodiversität, Zeitschrift für Umweltrecht, 2017, S. 387-392

BRILISAUER, KLAUS u.a., Cyanobacterial antimetabolite 7-deoxy-sedobeptulose blocks the shikimate pathway to inhibit the growth of prototrophic organisms, $\mathrm{Na}$ ture Communications 01.02.2019/10, Artikelnr. 545, abrufbar unter: https://www .nature.com/articles/s41467-019-08476-8/ (15.06.2019)

412 S. insbesondere die Vorschläge der Europäischen Ombudsfrau, (Fn. 208); die Forderungen des EP auf der Basis der Untersuchung des PEST-Ausschusses, (Fn. 95); sowie L.N. Vandenberg et al., Journal of Epidemiology and Community Health 2017/71, S. 613-618.

413 Brilisauer u.a., Nature Communications 2019/10, Artikelnr. 545 (01.02.2019), abrufbar unter www.nature.com (15.06.2019). 
BURGHARDT, PETER, Aus der Luft vergiftet-Pestizid-Sprübflugzenge in Argentinien, Süddeutsche Zeitung, 14.06.2012

BURTSCHER-SCHADEN, HELMUT; CLAUSING, PETER; ROBINSON, CLAIRE, Glyphosat und Krebs: Gekaufte Wissenschaft/Glyphosate and cancer, Buying science, März 2017, abrufbar unter: https://www.bund.net/fileadmin/user _upload_bund/publikationen/umweltgifte/Glyphosat_und_Krebs_Gekaufte_Wis senschaft_BUND_23032017.pdf (15.06.2019)

CHANG, ELLEN T.; DELZELL, ELIZABETH, Systematic review and meta-analysis of glyphosate exposure and risk of lymphohematopoietic cancers, Journal of Environmental Science and Health, Part B, 2016, Vol. 51, S. 402-434

DÖRR, OLIVER; GROTE, RAINER; MARAUHN, THILO (Hrsg.), EMRK/GG Konkordanzkommentar, Bd. I, 2. Aufl., Tübingen, 2013

DOUHAIRE, CAROLINE, EU-Pflanzenschutzmittelverordnung: Zur Berücksichtigung indirekter Biodiversitätseffekte bei der Zulassung von Pflanzenschutzmitteln, Zeitschrift für Umweltrecht, 2017, S. 393-399

FAIRCLOUGH, BOB; MAL PURAN; KERSTING, STEFAN, The economic relevance of glyphosate in Germany, Studie der Kleffman Group, Juli 2017, abrufbar unter: https://www.glyphosate.eu/system/files/sidebox-files/the_economic_relev ance_of_glyphosate_in_germany_24_08_2017.pdf (15.06.2019)

FENT, KARL, Umweltchemie - Toxikologie - Ökologie, Stuttgart, 4. Aufl., 2013

FOUCARD, STEPHANE; HOREL, STEPHANE, Monsanto papers, désinformation organisée autour du glyphosate, Le Monde, 04.10.2017

FOUCARD, STEPHANE, Les Monsanto Papers, à la base de la controverse sur le glyphosate, Le Monde, 11./13.08.2018

GREIM, HELMUT et al., Evaluation of carcinogenic potential of the herbicide glyphosate, drawing on tumor incidence data from fourteen chronic/carcinogenicity rodent studies, Critical Reviews in Toxicology, 2015, Vol. 45, S. 185-208

GUYTON, KATHRYN Z. et al., Carcinogenicity of tetrachlorvinphos, parathion, malathion, diazinon, and glyphosate, The Lancet Oncology, 2015, Vol. 16, S. 490-491

HALLMANN, CASPAR A. et al., More than 75 percent decline over 27 years in total flying insect biomass in protected areas, PlosOne, 18.10.2017, abrufbar unter: https://doi.org/10.1371/journal.pone.0185809 (15.06.2019)

HEAP, IAN, Global perspective of herbicide-resistant weeds, Pest Management Science, 2014, Vol. 70, S. 1306-1315

HENSEL, ANDREAS, Zwischen Wissen und Metaphysik - wo stebt das Vorsorgeprinzip im Streit um die Risikobewertung des Pflanzenschutzmittelwirkstoffs Glyphosat?, Zeitschrift für das gesamte Lebensmittelrecht, 2016, Vol. 4, S. 447-450

HOLLÄNDER, HEIKE; AMRHEIN, KLAUS, The Site of the Inbibition of the Shikimate Pathway by Glyphosate, Plant Physiology, 1980, Vol. 66, S. 823-829 
JOERES, ANNIKA, Hat Monsanto Wissenschaftler gekauft?, Zeit online, 11.10.2017

KAUS, VOLKER, Pflanzenschutzmittel-Wirkstoff Glyphosat-wissenschaftliche Unabhängigkeit auf dem Prüfstand!, Zeitschrift für Stoffrecht, 2016, S. 106-113

KLEIN, ALEXANDRA-MARIA, Auf diese Tiere können Sie bauen, Frankfurter Allgemeine Zeitung, 31.10.2018

KOOF, PETER, Pflanzenschutzmittel - Parallelhandel, gegenseitige Anerkennung und zonale Zulassung, Verwaltungspraxis deutscher Bebörden nach der VO (EG) $N r$. 1107/2009, Zeitschrift für Stoffrecht, 2016, S. $113 \mathrm{ff}$.

KROGMANN, LARS u.a., Neun-Punkte-Plan gegen das Insektensterben-Die Perspektive der Wissenschaft, Stuttgart, 2. Aufl., 19.10.2018

LERRO, CATHERINE C. et al., Organophosphate insecticide use and cancer incidence among spouses of pesticide applicators in the Agricultural Health Study, Occupational and Environmental Medicine, 2015, Vol. 72, S. 736-744

LIEBRICH, SILVIA, Was die Monsanto-Mails verraten, Süddeutsche Zeitung, 02.04.2017

MARKS, SIMON, Brussels moves goalposts on glyphosate: Commission plans to shift responsibility from Germany to France for evaluating controversial weedkiller, 18.07.2018, abrufbar unter: https://www.politico.eu/article/france-chosen-to-tak e-over-from-germany-in-assessing-glyphosate-monsanto-roundup/ (15.06.2019)

MICHALOPOULOS, SARANTIS, Andrinkaitis: 'Enough' with member states hiding behind the Commission on glyphosate, Euraktiv, 06.10.2017, abrufbar unter: https://www.euractiv.com/section/agriculture-food/news/andriukaitis-enough-w ith-member-states-hiding-behind-the-commission-on-glyphosate/ (15.06.2019)

NANDULA, VIJAY K. (Hrsg.), Glyphosate Resistance in Crops and Weeds: History, Development, and Management, New Jersey, 2010

NESLEN, ARTHUR, EU report on weedkiller safety copied text from Monsanto stu$d y$, The Guardian, 15.09.2017

PECHSTEIN, MATTHIAS; NOWAK, CARSTEN; HÄDE, ULRICH (Hrsg.), Frankfurter Kommentar EUV/GRC/AEUV, Bd. I, Tübingen, 2017

PECHSTEIN, MATTHIAS; NOWAK, CARSTEN; HÄDE, ULRICH (Hrsg.), Frankfurter Kommentar EUV/GRC/AEUV, Bd. III, Tübingen, 2017

PORTIER, CHRISTOPHER J. et al., Differences in the carcinogenic evaluation of glyphosate between the International Agency for Research on Cancer (IARC) and the European Food Safety Authority (EFSA), Journal of Epidemiology and Community Health, 2016, Vol. 70, S. 741-754

PORTIER, CHRISTOPHER J. et al., Open Letter: Review of Carcinogenicity of Glyphosate by EFSA and BfR, 27.11.2015, abrufbar unter: www.efsa.europa.eu/si tes/default/files/Prof_Portier_ldetter.pdf (15.06.2019) 
RANZENMAYR, ELKE, Glyphosat - eine nicht enden wollende Geschichte, Gesundheitliche Auswirkungen und rechtliche Folgen aufgrund des Einsatzes von Glyphosat, Saarbrücken, 2018

SCHÄFFER, ANDREAS u.a., in: Hacker Jörg (Hrsg.), Der stumme Frübling - Zur Notwendigkeit eines umweltverträglichen Pflanzenschutzes, Nationale Akademie der Wissenschaften - Leopoldina, Halle (Saale), Diskussion Nr. 16, 2018, S. 24 ff.

SCHMITT, CHRISTIAN, Ein Beitrag zu Produktstrafrechtlichen Haftungsrisiken von Herbiziden am Beispiel des Stoffes Glyphosat, Zeitschrift für Stoffrecht, 2018, S. 241-244

SOL BALBUENA, MARÍA et al., Effects of sublethal doses of glyphosate on honeybee navigation, Journal of Experimental Biology, 2015, Vol. 218, S. 2799-2805

SZÉKÁCS, ANDRÁS; DARVAS, BÉLA, Chapter 14 - Forty years with glyphosate, in: Abd El-Ghany Hasaneen, Mohammed Naguib (Hrsg.), Herbicides - properties, synthesis and control of weeds, InTech Europe, Rijeka, 2012, S. 247-84

TAMMS, LAURA; KONING, LAURIE ANNE; DE MOL, FREDERIKE; GEROWITT, BÄRBEL, Reaktion einer Population von Lolium multiflorum var. westerwoldicum auf wiederbolten Einsatz von Glyphosat, Julius-Kühn-Archiv, 2018, Vol. 458, S. $270 \mathrm{ff}$.

TARAZONA, JOSÉ VINCENTE et al., Glyphosate toxicity and carcinogenicity: a review of the scientific basis of the European Union assessment and its differences with IARC, Archives of Toxicology, 2017, Vol. 91, S. 2723-2743

THOMPSON, HELEN M. et al., Evaluating exposure and potential effects on honeybee brood (Apis mellifera) development using glyphosate as an example, Integrated Environmental Assessment and Management, 2014, Vol. 10, S. 463-470

VANDENBERG, L.N. u.a., Is it time to reassess current safety standards for glyphosate-based herbicides?, Journal of Epidemiology and Community Health, 2017/71, S. 613-618

WAGNER, CHRISTIAN, Ringen um Umweltinformationen - EuGH-Urteile zu grundlegenden Fragen der Arbus-Bestimmungen, Europäische Zeitschrift für Wirtschaftsrecht, 2017, S. 95-98

WEBER, STEFAN; BURTSCHER-SCHADEN, HELMUT, Detailed Expert Report on Plagiarism and superordinated Copy Paste in the Renewal Assessment Report (RAR) on Glyphosate, Januar 2019, abrufbar unter: www.greens-efa.eu/files/ doc/docs/298ff6ed5d6a686ec799e641082cdb63.pdf (15.06.2019)

WEGENER, BERNHARD, Umweltinformationsfreibeit - ernst genommen: Der Fall Glyphosat, Zeitschrift für Umweltrecht, 2014, Vol. 1, S. 32-36

WEGENER, BERNHARD, Kein „Mund auf - Augen zu“-Der freie Zugang zu Informationen über Emissionen in die Umwelt, Zeitschrift für Umweltrecht, 2017, Vol. 3, S. 146-152 